\title{
ESTABLISH CROWDSOURCING AS AN ORGANISATIONAL BUSINESS PROCESS: \\ A DESIGN SCIENCE APPROACH
}

\author{
by
}

\section{NGUYEN HOANG THUAN}

\author{
A thesis \\ submitted to the Victoria University of Wellington \\ in fulfilment of the requirements for the degree of \\ Doctor of Philosophy \\ in Information Systems
}

Victoria University of Wellington

2016 
"Welcome to the age of the crowd".

(Howe, 2006b, p. 1) 


\section{Abstract}

Crowdsourcing can be an efficient organisational strategy to harness innovation and agility by distributing work to Internet users. As crowdsourcing is different from other business strategies, organisations are often unsure about how to structure crowdsourcing as a business process. We identify two challenges: first, crowdsourcing has been perceived as a one-off endeavour and a comprehensive repeatable crowdsourcing process has not been defined yet. Second, while organisations need a solid knowledge base in order to integrate crowdsourcing with their business processes, the domain knowledge remains unstructured, scattered, and sometimes conflicting. Together, these challenges indicate that crowdsourcing needs to evolve from an immature form towards a more repeatable business process.

The research adopts the design science paradigm and follows four research stages. We first synthesise existing knowledge in the domain, which identifies twelve repeatable building blocks of crowdsourcing processes. Second, we define a model of business process crowdsourcing (BPC). The model has seven processes organised in three stages: decision to crowdsource, process design, and technical configuration. The model was empirically evaluated using two existing crowdsourcing projects. The results suggest the usefulness of the model for structuring crowdsourcing processes.

Third, we turn the BPC model into a heavyweight ontology that consolidates the domain knowledge. The ontology captures the following domain concepts: business processes, activities, data entities, data attributes, and their hierarchical relationships. It also captures decision-making relationships. We evaluated the ontology by triangulation. The findings are that the ontology provides a high coverage and clarity of the domain.

Finally, based on the ontology, a decision tool is developed. The tool provides advice to make informed decisions about BPC establishment. Using experiments and focus groups to evaluate the tool, the quantitative and qualitative results confirm its utility and show that the tool improves decision-making performance. 
The dissertation contributes to the body of knowledge in several ways. It promotes and conceptualises crowdsourcing as an organisational business process. It offers a process model, ontology, and decision tool for BPC. It also provides empirical results about the use of the decision tool. By doing so, we hope that the dissertation will motivate organisations to further assimilate crowdsourcing.

Keywords. Business Process Crowdsourcing, Crowdsourcing, Decision Support System, Design Science, Ontology 


\section{Acknowledgements}

My first acknowledgement is to my supervisors, Associate Professor Pedro Antunes and Dr David Johnstone. You not only guided me through the development of the $\mathrm{PhD}$ project but also showed me how to structure, sharpen, and publish research results. Your invaluable support, encouragement, and ideas contribute to make it possible for me to complete this work. You have always been there when I ask for five-minute meetings that may end up with one-hour discussions.

I would like to thank my family for their support and understanding. Thanks to my parents and my mother-in-law, who took over my responsibilities when I was absent from home. Thanks to my wife, Xuan Trang, for love, support, and motivation. Thanks, too, to my son, Hoang Bach, providing me with the strength to complete my $\mathrm{PhD}$.

A special thank you to the School of Information Management (SIM) academics, staff, and administrative team. You provided all necessary support for researching, tutoring, marking, and lecturing. Special thanks to Dan Dorner, Usha Varatharaju, Dion Peszynski and Jean Grant for your valuable support. My sincere thanks to the New Zealand ASEAN Scholarship. I am honoured to be one of the scholarship holders. Thanks to the Can Tho University of Technology; you were there to assist me during my data collection. Thanks also to Penelope Trought for her proof reading.

My sincerest thanks to the examiners: Professor Frada Burstein, Professor Gert-Jan De Veerde, and Dr Brenda Chawner for extraordinarily insightful and helpful feedback.

Thanks you to each individual who participated in the data gathering of this research. You provided me valuable insight into the topic that I have enjoyed studying.

Finally, thanks to my friends who helped balance my research life and my other life. In particular, thanks to the SIM PhD cohorts, flatmates, and members of Vietnamese Tennis Club of Wellington. You have brought encouragement and fun to my life. 


\section{Table of Contents}

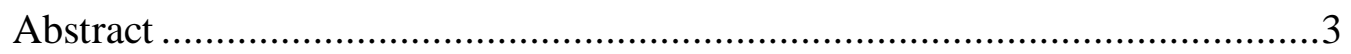

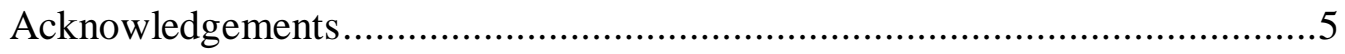

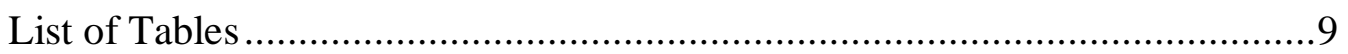

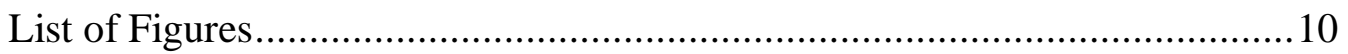

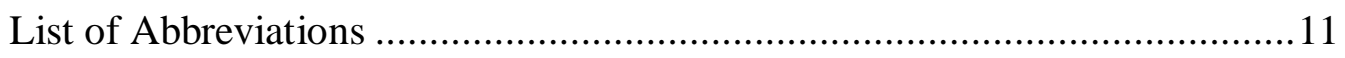

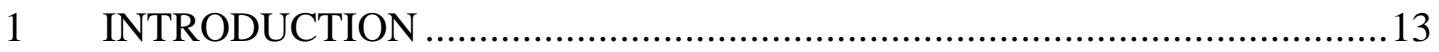

1.1 Research Problem and Objectives .............................................. 16

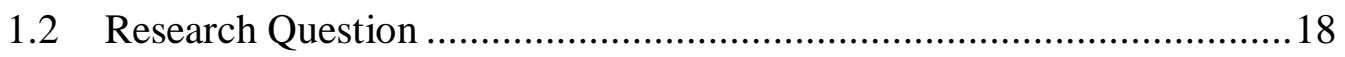

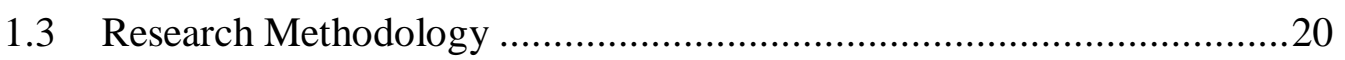

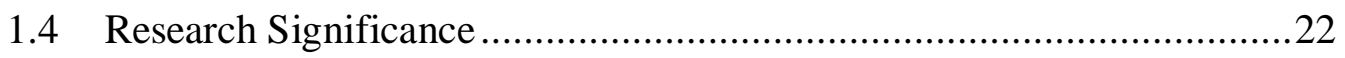

1.5 Dissertation Structure..................................................................23

1.6 List of Publications ........................................................................24

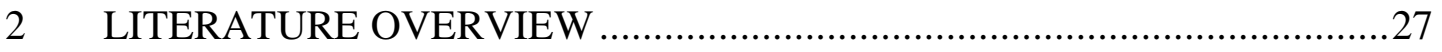

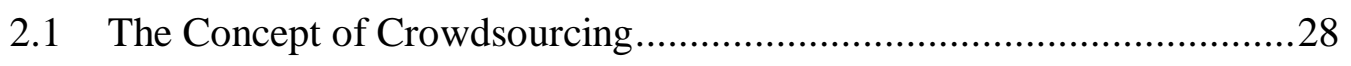

2.1.1 Main Idea behind Crowdsourcing …......................................28

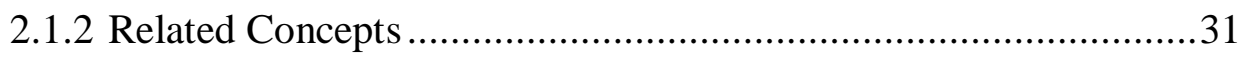

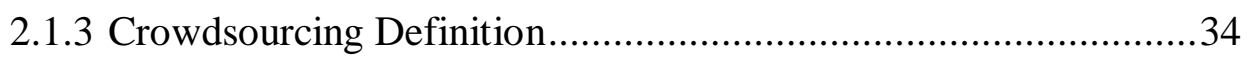

2.2 Crowdsourcing Classifications, Taxonomies, and Typologies ................38

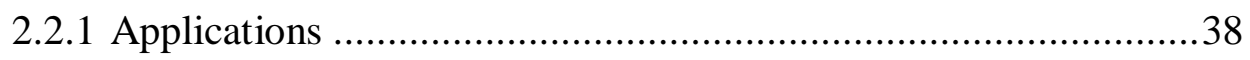

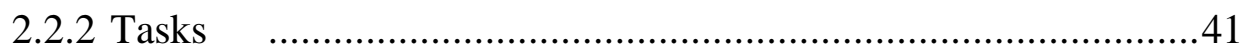

2.2.3 Members of the Crowd .......................................................4 43

2.2.4 Platforms ......................................................................... 44

2.3 A Process Perspective on Crowdsourcing ........................................4 47

2.3.1 Crowdsourcing Process .......................................................4 47

2.3.2 Business Process Crowdsourcing ............................................52

2.4 An Analytical Framework of BPC .......................................................55

2.4.1 Decision to Crowdsource ........................................................56

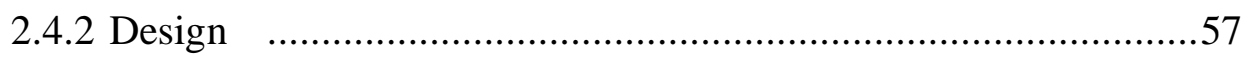

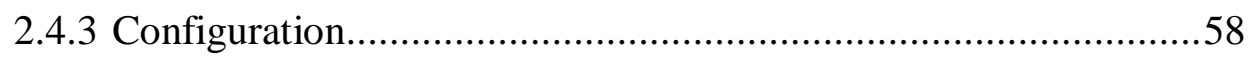

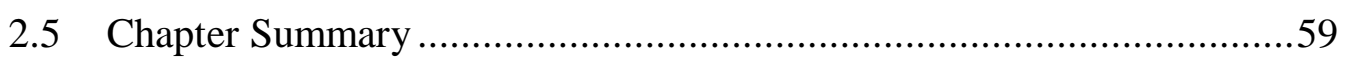

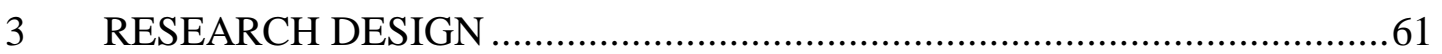

3.1 Design Science Paradigm.................................................................61

3.2 Design Methods and a Need of a Design Method for Emerging

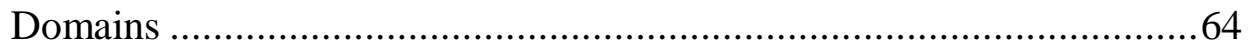

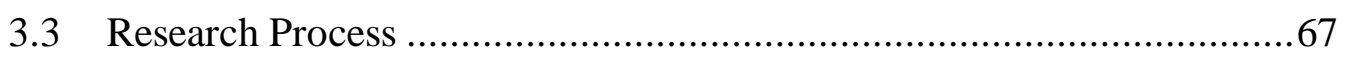




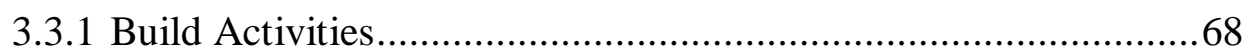

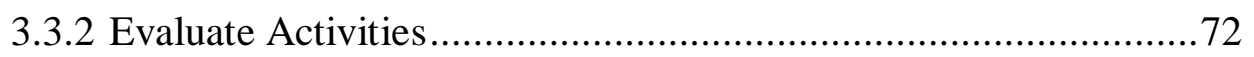

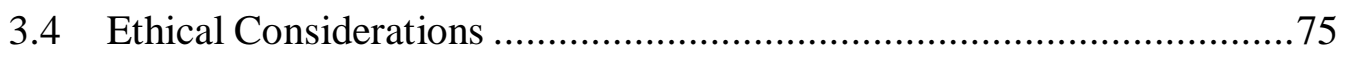

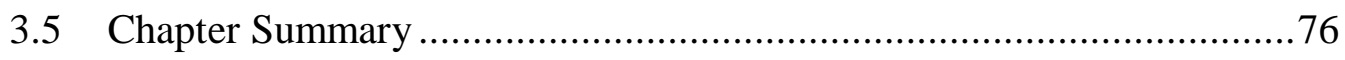

4 THE FOUR RESEARCH STAGES OF BUILD AND EVALUATE...............77

4.1 Stage 1: Scoping Knowledge Sources ...................................................78

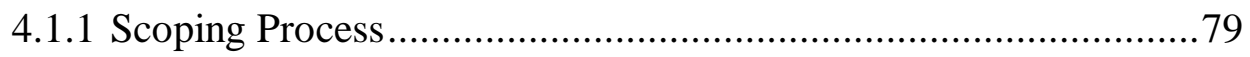

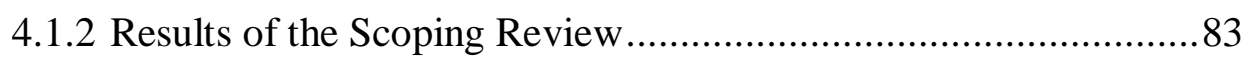

4.1.2.1 Building Blocks of BPC ..................................................... 84

4.1.2.2 Factors Influencing the Decision to Crowdsource................... 85

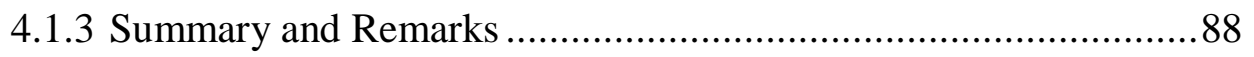

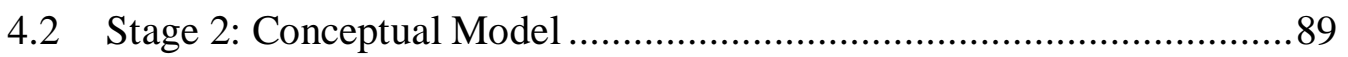

4.2.1 A Process Model for BPC Establishment ......................................89

4.2.1.1 A Framework Supporting the Decision to Crowdsource......... 94

4.2.2 Evaluation of the Model: Case Studies..........................................101

4.2.2.1 Overview of the Approach.................................................... 101

4.2.2.2 Case Study Design ........................................................... 102

4.2.2.3 Case Study Results........................................................... 105

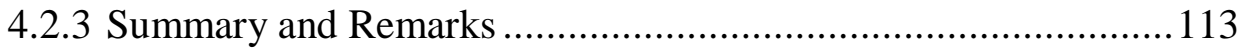

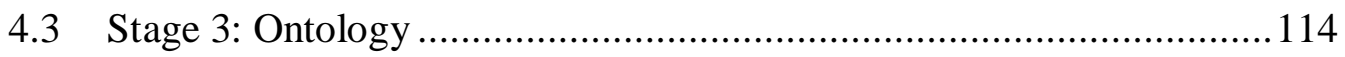

4.3.1 Building a BPC Ontology ………………………...................114

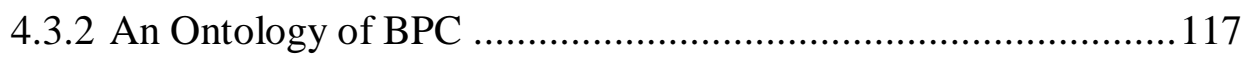

4.3.2.1 Salient Concepts................................................................. 117

4.3.2.2 Hierarchy Relationships ..................................................... 119

4.3.2.3 Decision Making Relationships and Business Rules ............. 122

4.3.3 BPC Ontology Evaluation: Triangulation.....................................124

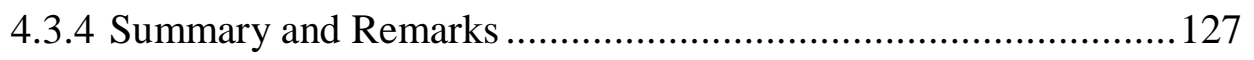

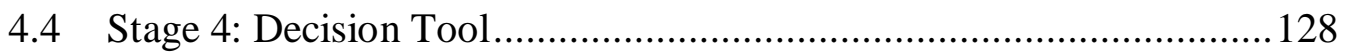

4.4.1 Architecture and Decision Tables.............................................130

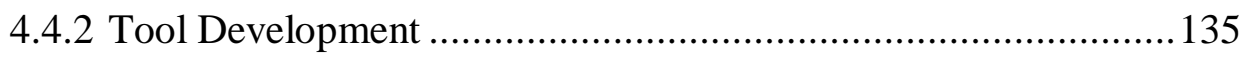

4.4.2.1 The First Prototype...................................................... 135

4.4.2.2 Evaluation: Pilot Experiment............................................... 138

4.4.2.3 Feedback for Revision and the Second Prototype ................. 140

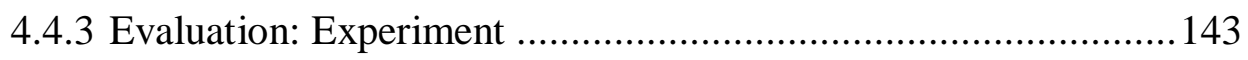

4.4.3.1 Experimental Overview......................................................... 143

4.4.3.2 Experimental Design .......................................................... 144

4.4.3.3 Experimental Results......................................................... 148

4.4.4 Evaluation: Focus Group ……………………………….........154 


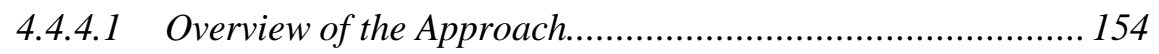

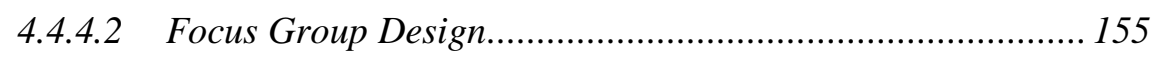

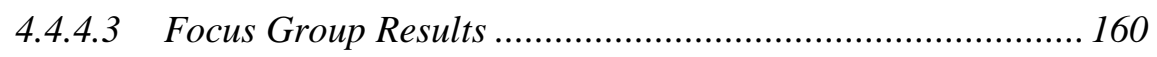

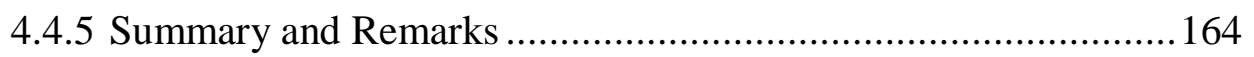

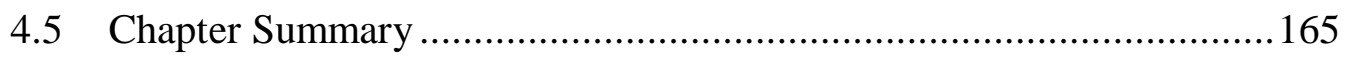

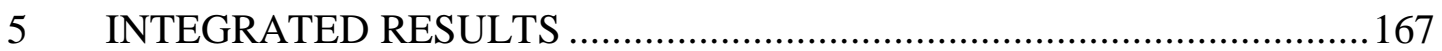

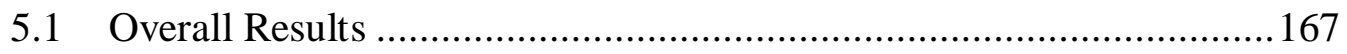

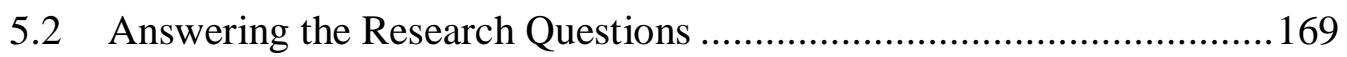

5.3 Chapter Summary …................................................................. 177

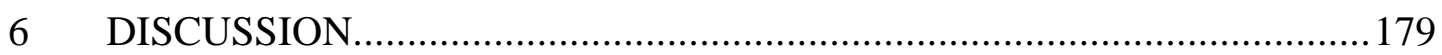

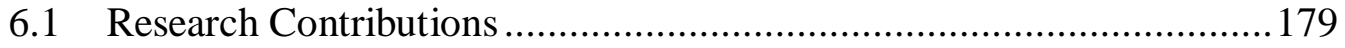

6.1.1 A New Approach for Establishing Crowdsourcing as an

Organisational Business Process ............................................... 179

6.1.2 The Importance of the Ontology ............................................. 180

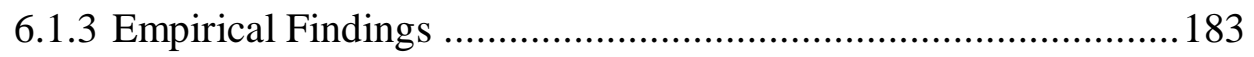

6.1.4 Progression of Business Process Crowdsourcing ...................... 185

6.2 Other Contributions to Design-Science Knowledge Base ................... 187

6.2.1 Design Product - Three Outcome Artefacts .............................187

6.2.2 Design Process - A Design Method for Emerging DSS

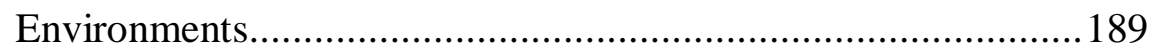

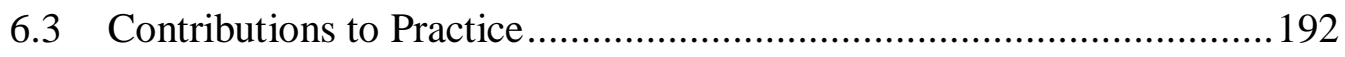

6.4 Limitations of the Research............................................................ 193

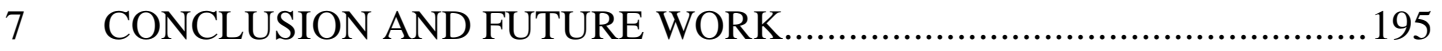

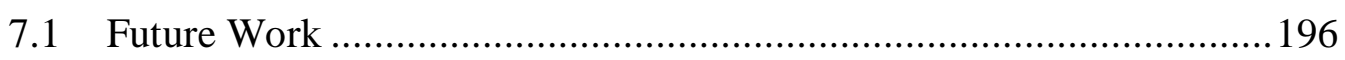

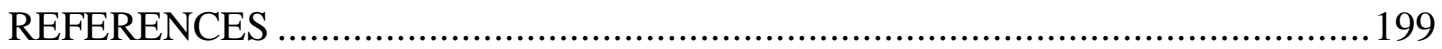

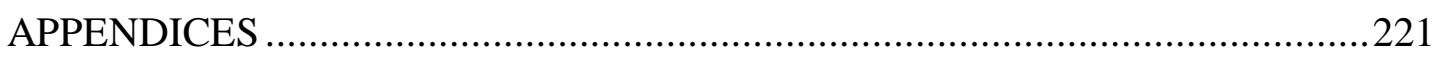

Appendix A. Crowdsourcing Classifications …..............................221

Appendix B. $\quad$ Ethics Approvals ..................................................222

Appendix C. Coding Form.........................................................237

Appendix D. Interview Protocol.................................................238

Appendix E. Ontology Vocabulary ..............................................24

Appendix F. $\quad$ Experimental Sheet .................................................242

Appendix G. Partial Focus Group Script ......................................246 


\section{List of Tables}

Table 2.1. Main differences between crowdsourcing and related concepts ...........33

Table 2.2. Typology of crowdsourcing applications (Zhao \& Zhu, 2014).............40

Table 2.3. Examples of crowdsourcing task types ............................................42

Table 3.1. Structure for summarising main concepts of BPC (adapted from Webster

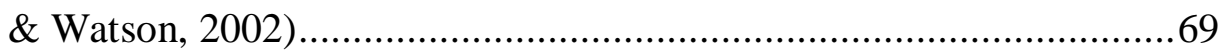

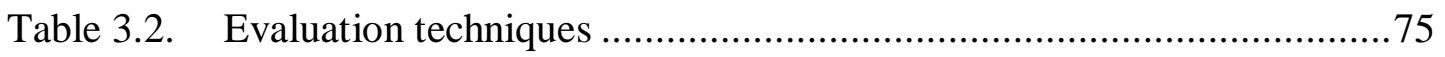

Table 4.1. Crowdsourcing sources on the eight bibliographic databases ................80

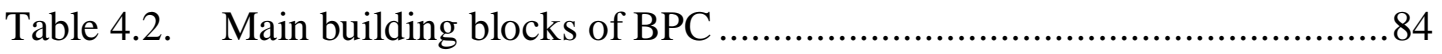

Table 4.3. Factors that influence the decision to crowdsource .............................86

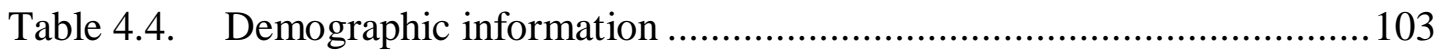

Table 4.5. Validity of the case studies............................................................ 104

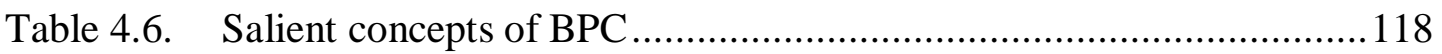

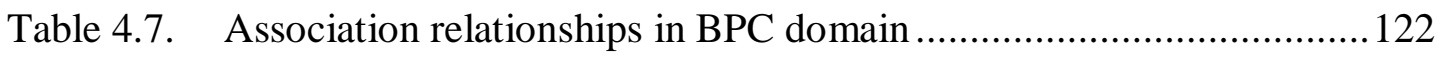

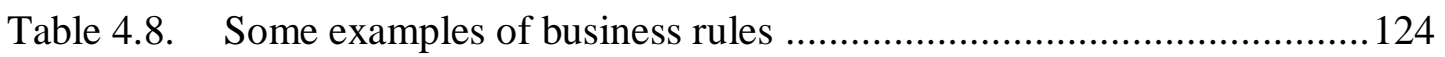

Table 4.9. Decision table: Task properties .......................................................131

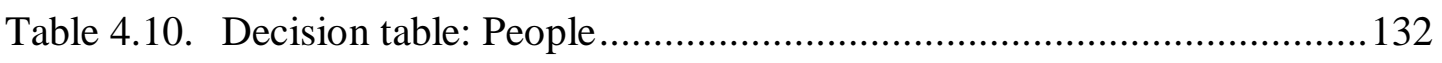

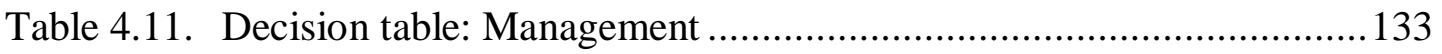

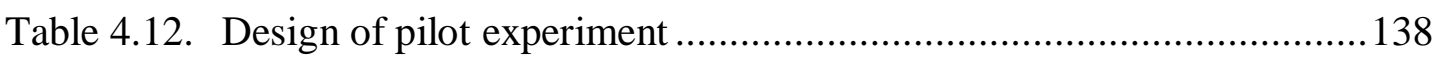

Table 4.13. Pilot experiment: Score frequency in the two exercises .....................139

Table 4.14. Descriptive data and p-value: Exercise 1 .........................................139

Table 4.15. Descriptive data and p-value: Exercise 2 .......................................139

Table 4.16. Experimental design: Swapping roles between the two groups ...........145

Table 4.17. Numbers of participants per session (chronological order) ................ 145

Table 4.18. Descriptive results of six experimental sessions ................................148

Table 4.19. Comparison between groups using the tool and without the tool........149

Table 4.20. Results of Mann-Whitney tests on exercise 1 ...................................150

Table 4.21. Results of Mann-Whitney tests on exercise 2 ...................................151

Table 4.22. Statistics of usefulness perceived by experimental participants...........152

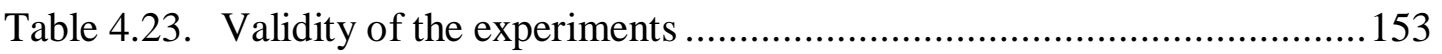

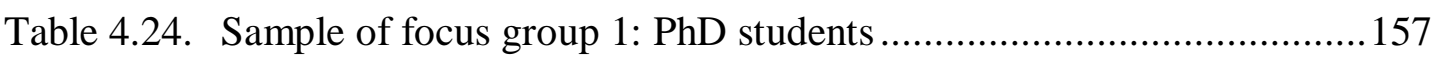

Table 4.25. Sample of focus group 2: Crowdsourcing experts................................. 157

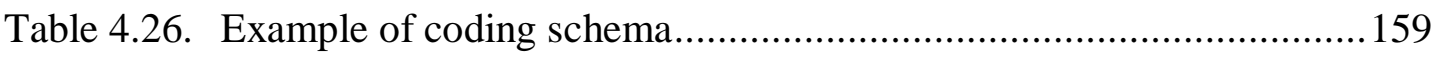

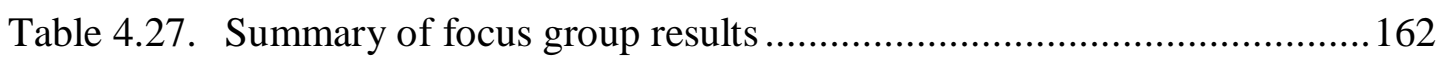

Table 5.1. Summary of results that answer the research questions .....................170

Table 6.1. Summary of the two research projects using the SCOA language .......192 


\section{List of Figures}

Figure 1.1. The Flag Consideration project statistics ........................................ 14

Figure 2.1. Layered framework for the literature review...................................22

Figure 2.2. Evolution of crowdsourcing definitions ............................................. 35

Figure 2.3. Three-stage analytical framework .................................................56

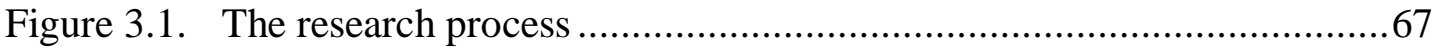

Figure 4.1. Steps and summary of results of the scoping knowledge sources .........79

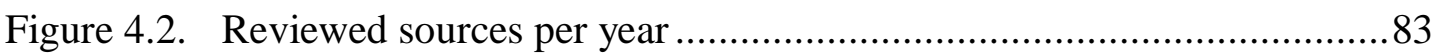

Figure 4.3. A process model for BPC establishment ..........................................90

Figure 4.4. A framework that supports the decision to crowdsource ......................94

Figure 4.5. Activities of Crowd Tagging (CT) ................................................ 105

Figure 4.6. Activities of Logo Design Contest (LDC) ...................................... 105

Figure 4.7. Ontology capture schema (adapted from Rockwell et al., 2010) ........115

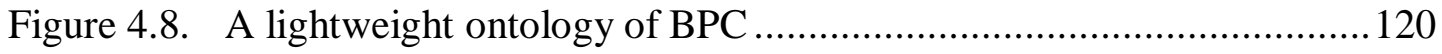

Figure 4.9. Positive and negative influence relationships................................... 123

Figure 4.10. Ontological version generated by OntoGen (OntoGen was developed by Fortuna et al., 2007) ...................................................................... 126

Figure 4.11. Tool architecture (adapted from Holsapple, 2008) ..........................130

Figure 4.12. Tool 1: The decision to crowdsource ............................................ 136

Figure 4.13. Tool 2: Process design of BPC establishment ................................... 136

Figure 4.14. Abstract views of Tool 1 and Tool 2 ............................................137

Figure 4.15. Pilot experiment: Pictures of Group A (left) and Group B (right) ......138

Figure 4.16. Second prototyping: Decision to crowdsource (Tool 1) ..................... 142

Figure 4.17. Second prototype: Process design (Tool 2) ...................................... 142

Figure 4.18. Distributions of Group A and Group B: Exercise 1 .........................150

Figure 4.19. Distributions of Group A and Group B: Exercise 2 .........................151

Figure 4.20. Focus group procedure (adapted from Tremblay et al., 2010) .............155

Figure 5.1. Interrelated knowledge in structuring BPC ................................... 167

Figure 5.2. Interrelated yet different levels of abstraction: Outcome artefacts...... 168

Figure 6.1. Progression of business process crowdsourcing ............................... 185

Figure 6.2. Research design products (adapted from Gregor \& Hevner, 2013) .... 187

Figure 6.3. The SCOA method (Thuan et al., 2015a)........................................190 


\section{List of Abbreviations}

$\begin{array}{ll}\text { AMT } & \text { Amazon Mechanical Turk } \\ \text { API } & \text { Application Programming Interface } \\ \text { BPC } & \text { Business Process Crowdsourcing } \\ \text { BPM } & \text { Business Process Management } \\ \text { BPMN } & \text { Business Process Model and Notation } \\ \text { BPO } & \text { Business Process Outsourcing } \\ \text { DTN } & \text { Design Theory Nexus } \\ \text { DSS } & \text { Decision Support System } \\ \text { GUI } & \text { Graphic User Interface } \\ \text { IM } & \text { Industrial Management } \\ \text { IP } & \text { Intellectual Property } \\ \text { IS } & \text { Information Systems } \\ \text { IT } & \text { Information Technology } \\ \text { R\&D } & \text { Research and Development } \\ \text { SDLC } & \text { System Development Life Cycle }\end{array}$




\section{INTRODUCTION}

To open this dissertation, let us imagine how excellent organisations could be if they were not limited to human resource constraints and could access labour and skills on demand. In fact, this image has now come true thanks to crowdsourcing. Crowdsourcing is an emerging sourcing strategy that utilises Internet users through an open call to perform tasks (Howe, 2006b). This strategy has been used by many organisations for harnessing on-demand workforce, external expertise, and creativity. The development of crowdsourcing has been gathering momentum in terms of growth revenues and adoption. For instance, in 2011 crowdsourcing revenue reached $\$ 375.70$ million, while increasing $74.7 \%$ within one year ${ }^{1}$; and between 2013 and 2014 the adoption of crowdsourcing by leading organisations like Microsoft and Google increased $48 \%{ }^{2}$.

The fundamental idea behind the crowdsourcing strategy is that an organisation (which could be a company, non-profit organisation, or government) defines tasks and broadcasts them online to the crowd, who voluntarily undertake these tasks in an individual or collaborative way. When completing these tasks, individuals in the crowd submit their work back to the organisation, which assesses the work quality and may provide incentives or individual compensations (Durward, Blohm, \& Leimeister, 2016; Zhao \& Zhu, 2014). It is worth mentioning that the process normally unfolds through crowdsourcing platforms like InnoCentive ${ }^{3}$ and Amazon Mechanical Turk (AMT) ${ }^{4}$.

Among the organisations adopting crowdsourcing, the most interesting, and maybe biggest one, was the New Zealand government. In 2015, the government established a $\$ 25.7$ million project, named the Flag Consideration Project ${ }^{5}$, which looked for the future flag of New Zealand. Interestingly, this project relied neither solely on design companies nor on professional designers, rather it had been opened to everybody. More precisely,

\footnotetext{
1 Source: Massolution White Paper: http://www.lionbridge.com/files/2012/11/Lionbridge-WhitePaper The-Crowd-in-the-Cloud-final.pdf

${ }^{2}$ Source: eYeka Trend Report: http://eyeka.pr.co/99215-eyeka-releases-the-state-of-crowdsourcing-in2015-trend-report

${ }^{3}$ Source: InnoCentive: http://www.innocentive.com/

${ }^{4}$ Source: Amazon Mechanical Turk: https://www.mturk.com/mturk/welcome

${ }^{5}$ Source: the Flag Consideration Project: https://www.govt.nz/browse/engaging-with-government/the-nz-flagyour-chance-to-decide/
} 
the project proposed an open call for all New Zealanders to create flag designs and ultimately they would decide on the future flag of New Zealand. The project started in May 2015 and received more than 10,000 flag designs after three months. These designs were then shortlisted into four alternatives. The shortlisted designs and the current flag were voted by New Zealanders through a two-round referendum in December 2015 and March 2016. More than two million New Zealanders voted in the referendum with the final decision to retain the current flag. For the project, crowdsourcing allowed the New Zealand government to harness creativity and design expertise, to know what New Zealanders stand for, and to collectively make the final decision. In this manner, the crowdsourcing project has already been very successful, receiving a huge number of submissions and attracting significant public attention, as shown in Figure 1.1.

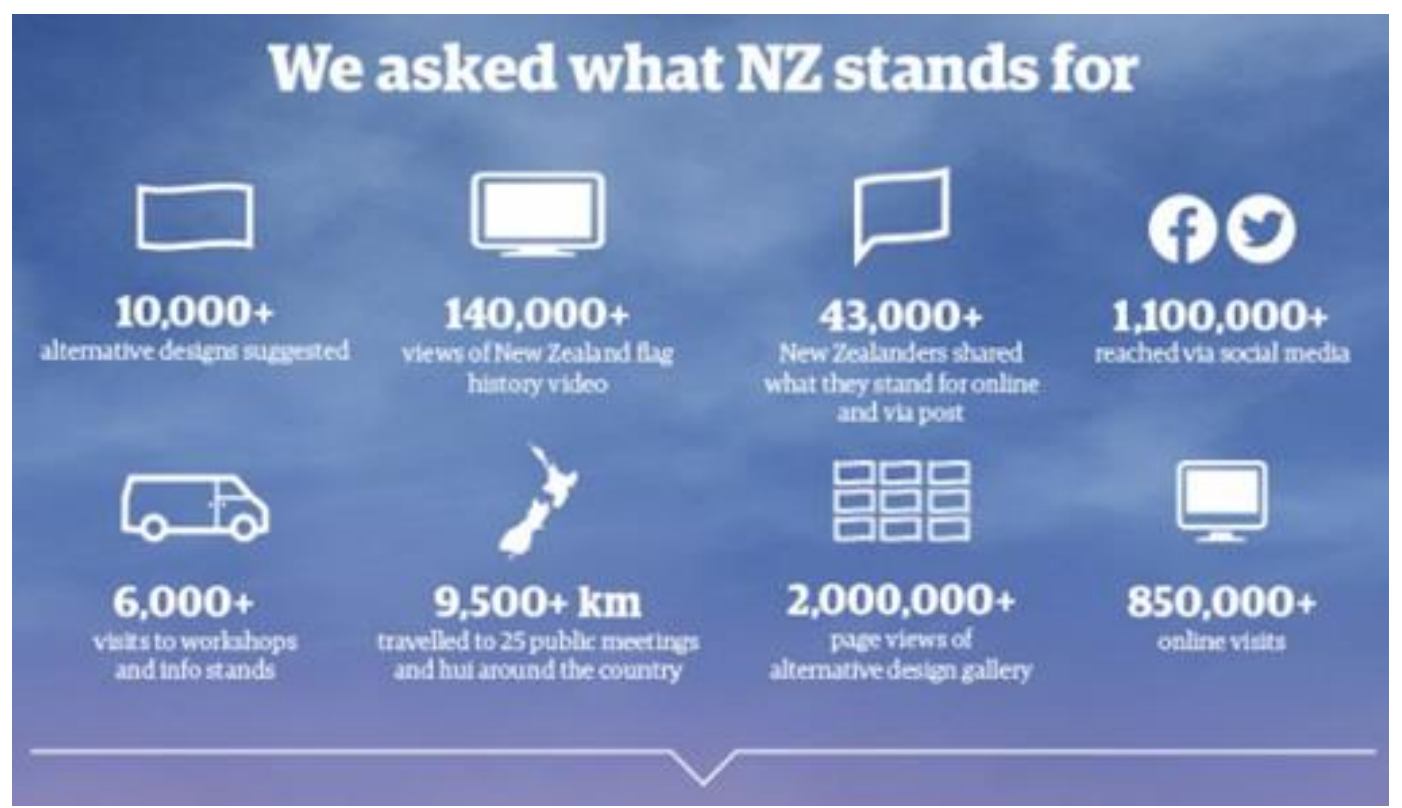

Figure 1.1. The Flag Consideration project statistics ${ }^{6}$

Though crowdsourcing has been used to harness creative design skills, as in the Flag Consideration Project, the use of crowdsourcing is not limited to design or to attracting public attention. Organisations have been adopting the crowdsourcing strategy for varied purposes. For instance, BMW, Boeing, Colgate-Palmolive, Procter and Gamble, and Netflix have all used crowdsourcing for research and development (R\&D) activities. These organisations have published difficult $R \& D$ issues online and have called for

\footnotetext{
${ }^{6}$ Source: the Flag Consideration Project: http://ndhadeliver.natlib.govt.nz/ArcAggregator/arcView/frameView/IE25848623/https:/www.govt.nz/browse/engagi ng-with-government/the-nz-flag-your-chance-to-decide/
} 
innovative solutions from the crowd. Also utilising the crowd, Lego, Threadless, and Starbucks have used crowdsourcing for gathering customers' ideas, which generates innovation and increases the ties with their customers. Other organisations rely on the crowd for performing their day-to-day activities, such as transcribing texts, gathering customer surveys, and processing information. All these examples, and many others ${ }^{7}$, illustrate how organisations have successfully utilised the crowd for sourcing organisational tasks.

The power of crowdsourcing lies in its ability to distribute work beyond the organisational boundaries and harness a variety of business endeavours. By adopting a crowdsourcing strategy, organisational tasks can be accomplished by Internet users, independent from time and geographic constraints. Crowdsourcing can also harness expertise, problem solving and creativity from outside of the organisational boundaries, and serve as a project stimulator, as shown in the Flag Consideration Project. Furthermore, it is a relatively costeffective way of doing work because the crowd can be assembled on demand and in many cases are voluntary, or receive only a few dollars per task (Boudreau \& Lakhani, 2013; Brabham, 2008a; Rosen, 2011; Saxton, Oh, \& Kishore, 2013). All in all, virtually limitless workforce, varied skills and relatively low costs make crowdsourcing a valuable sourcing strategy for organisations.

Bringing value to organisations, crowdsourcing at the same time changes the way organisations manage and conceive work. Through crowdsourcing, organisations have been able to access human resources from virtually everywhere. This openness requires organisations to re-define their organisational boundaries, and more importantly to effectively establish conduits between internal and external activities across the boundaries (Majchrzak \& Malhotra, 2013; Tranquillini, Daniel, Kucherbaev, \& Casati, 2015). Furthermore, crowdsourcing has changed the way organisations design work structures. Different from traditional structures, which establish a stable and top-down hierarchy, crowdsourcing promotes a dynamic, bottom-up structure where professional knowledge, decision making, problem solving, and supervisory control can be assigned to members of the crowd (Brabham, 2013; Kittur et al., 2013). These changes properly reflect a new business model fostered by the Internet era.

\footnotetext{
${ }^{7}$ https://en.wikipedia.org/wiki/List_of_crowdsourcing_projects
} 
However, in this new crowdsourcing business model, there is a missing component from the above successful stories. It is clear about what organisations can possibly achieve through crowdsourcing, but how they adapt their traditional business structure to establish a crowdsourcing strategy is not yet clear. Motivated by this 'how to' challenge, coupled with the increasing popularity of crowdsourcing as a business practice, this dissertation aims at supporting organisations successfully establishing the crowdsourcing strategy.

\subsection{Research Problem and Objectives}

The success of the crowdsourcing strategy depends on how organisations establish crowdsourcing processes, which structure a set of activities necessary to operationalise the strategy. Well-planned and dedicated processes are assumed not only to produce better crowdsourcing results, but also to deploy the strategy faster on top of existing crowdsourcing platforms (Tranquillini et al., 2015). In contrast, an ad hoc process affects organisations due to failed outputs, the need for re-planning and the waste of organisational resources (Muhdi, Daiber, Friesike, \& Boutellier, 2011; Rouse, 2010). Furthermore, understanding the crowdsourcing process allows us to define its information flows, which is an antecedent for crowdsourcing implementation. All in all, the crowdsourcing process plays a central role in the crowdsourcing strategy.

Given the important role, researchers have already begun to investigate the crowdsourcing process. By and large, research on crowdsourcing processes can be classified into two views: low and high levels of granularity. With low granularity, a large number of studies have already investigated several parts of the crowdsourcing process. Yet, most of them tend to focus on individual aspects of the crowdsourcing process. The ad hoc nature of these studies has been recently highlighted in the literature (Thuan, Antunes, Johnstone, \& Ha, 2015b; Zhao \& Zhu, 2014). The challenge is not about their usefulness due to lack of replications, but instead there is no collective cohesiveness. That is, there are few contributions towards a holistic view of the crowdsourcing process. Rather, many of them regard crowdsourcing as a one-off process. As a result, this group of studies have left us with unstructured, scattered, and sometimes conflicting knowledge, which hinders our ability to build a dedicated repeatable crowdsourcing process. 
Recently, some research efforts have adopted a high level of granularity when analysing and conceptualising the crowdsourcing process. Trying to paint an overall picture of the crowdsourcing process (Grace et al., 2015; Muhdi et al., 2011; Stol \& Fitzgerald, 2014), they tend to be more focused on high level concepts, and thus face significant gaps regarding explanation of how to effectively establish the crowdsourcing process in detail. Furthermore, as they are mainly exploratory efforts, further research is needed to validate and empirically test their propositions before they can be used in practice. As a result, the domain is still lacking a solid knowledge base that organisations can rely upon to establish the crowdsourcing process.

Together, these challenges indicate that the crowdsourcing process is immature, with a lack of a solid knowledge base, unstructured sets of knowledge sources, and a dominant one-off perspective. This immaturity has prevented organisations from establishing crowdsourcing as a repeatable organisational process. Given that, the central research problem addressed in this dissertation is as follows:

Research problem: The immature approach to crowdsourcing, characterised by a lack of a solid knowledge base and unstructured sets of knowledge sources, has prevented organisations from establishing repeatable crowdsourcing processes.

Addressing this problem enables crowdsourcing to evolve from an unstructured, immature form towards a more repeatable process. To this end, it seems that reconciling the low and high levels of granularity provides a more integrated picture of the crowdsourcing process. That is, we can understand both the details of crowdsourcing processes and their abstract coordination. This integrated view suggests the use of a business process lens, which has scarcely been adopted in the crowdsourcing field.

This research will investigate crowdsourcing using a business process lens, essentially decomposing complex processes into a set of independent, yet coordinated, activities (van der Aalst \& Hee, 2004). This lens allows us to analyse independent crowdsourcing elements and coordinate them into an integrated crowdsourcing process. We designate this particular view as Business Process Crowdsourcing (BPC), a term that was first coined by La Vecchia and Cisternino (2010). Based on the BPC view, we seek to consider crowdsourcing as a repeatable business process, overcoming the one-off viewpoint. Further, BPC that relies on both high and low levels of granularity enables us to analyse 
and structure existing knowledge sources, and to build a solid knowledge base. Consequently, we expect BPC to move crowdsourcing towards a more mature form.

Given the discussion, and that BPC is emergent yet promising to address the research problem, the research objectives of the dissertation are set around this concept. This means the research objectives can be expressed as follows:

RO1: To conceptualise BPC considering crowdsourcing as a repeatable business process

RO2: To construct a solid knowledge base of BPC that organises the unstructured knowledge in the domain

If we achieve RO2, that is, we successfully construct a solid knowledge base of BPC, computer-based decision support can be further developed for assisting organisations establishing their business processes based on crowdsourcing. This leads to the next and final research objective of the dissertation.

RO3: To construct a decision tool supporting organisations in establishing BPC

\subsection{Research Question}

Research questions define the scope of the research. Yin (2013a) provides an excellent formulation to form research questions, which is a combination of substance and form. Substance refers to what the study is about, which is the research phenomenon. Form is the type of questions that then directly link to the research methods. An exemplar of the form is that case study methods are largely suitable to the 'why' and 'how' questions that address the operational links of events over time (Yin, 2013a).

In this dissertation, specifying the form of research questions is challenging. This is because the dissertation has adopted a design science paradigm as presented below, but there is little guidance on formulating research questions in the design science literature. Facing this challenge, the dissertation examines the main aspects of design science in order to look for a common form of research questions. Design science consists of two major complementary aspects: design process and design product (Hevner, March, Park, $\&$ Ram, 2004). The former focuses on the process, moving from a particular problem to 
a solution. Thus its main question is 'how to' (Gregor, 2006), which addresses the rationale of solving particular problems. The latter focuses on the outcome artefacts. Thus, its main question is 'what'; mostly 'what are the characteristics/constructs of the effective artefacts' (Kohlborn, 2012). Although other question forms can be found in design science, the 'what' and 'how to' are among the most popular forms, and are also suitable with the research objectives to 'conceptualise' and 'construct'. Consequently, they are used to formulate the research questions in this dissertation.

We now define the research questions of the dissertation, combining its substance and form (Yin, 2013a). The substance has been reflected through the three above research objectives set around BPC. The two identified forms of design-science research questions, 'what' and 'how to', are adapted regarding the particular objectives. In particular, RO1 seeks to conceptualise BPC. This requires us to understand the building blocks of BPC and to structure them for BPC conceptualisation. We define the term 'building blocks', aligning to Osterwalder (2004), as common decomposed elements that can be combined to describe the overarching concept. Consequently, the underlying research questions are developed.

RQ1: What are the main building blocks of BPC that can be identified in the domain?

RQ2: What are the structures of the identified building blocks for conceptualising $B P C$ ?

$\mathrm{RO} 2$ is aimed at constructing a solid knowledge base of BPC. The literature has suggested that domain ontologies can consolidate knowledge and construct knowledge bases (Kohlborn, 2012; Miah, 2008). In this vein, we target at constructing a domain ontology of BPC. Although the target is clear, what is unclear is how we can move from the unstructured knowledge sources to a domain ontology of BPC. Thus, the next research question is:

RQ3: How to construct a domain ontology of BPC that organises the unstructured knowledge sources in the domain?

The research question formulating RO3 is straightforward.

RQ4: How to construct a decision tool supporting organisations in establishing BPC? 
These research questions are non-causal, since they do not have a formulation of causal relationships among a set of variables (Miles, Huberman, \& Saldaña, 2014). Indeed, the dissertation purposefully formulates these non-causal questions for three reasons. First, the questions have been formulated according to the chosen design science paradigm. Second, the dissertation focuses on supporting organisations to establish BPC, which leads us to develop the research questions more about how to model, enable, construct, and support. Finally, the non-causal research questions are popularly formed in design science dissertations (Kohlborn, 2012; Miah, 2008; Osterwalder, 2004). All in all, these four non-causal research questions guide this study.

\subsection{Research Methodology}

This study adopts a design science paradigm (Hevner et al., 2004). Gradually being embraced as an important and solid research paradigm in the Information Systems (IS) discipline, design science focuses on constructing innovative solutions and shows how to do that effectively (Gregor \& Hevner, 2013). Consequently, it is a good fit with the 'construct' and 'support' focus of the study. This adoption is further appropriate as design science emphasises a rigorous approach to advance current knowledge on design problems (Hevner et al., 2004). The knowledge advancement is extremely necessary with unstructured domains like crowdsourcing.

A key principle of design science research is that it must be founded on a rigorous knowledge foundation, which according to Hevner and Chatterjee (2010) comprises three types: 1) scientific theories; 2) meta-artefacts; and 3) experience and expertise. With the ad hoc nature of the domain, we could not find a prevailing crowdsourcing theory or meta-artefact on which to base the research (Thuan et al., 2015b; Zhao \& Zhu, 2014). Rather than theory, a relatively large amount of experience and expertise exists in the form of individual knowledge sources, including case studies, expert opinion, and various types of design and prototyping developments. Thus, the current study focuses on the third type of knowledge foundations set by Hevner and Chatterjee (2010): building a knowledge base from individual knowledge sources predominant in the domain. 
Another key principle of design science is that research has to follow an appropriate design method guiding the research activities. For this study, we follow a design method comprising four research stages. First, this method systematically analyses and scopes individual knowledge sources in the domain for understanding the main building blocks of BPC (Okoli, 2015; Paré, Trudel, Jaana, \& Kitsiou, 2015). Second, the method proposes a conceptual model using the identified building blocks to conceptualise the phenomenon (Webster \& Watson, 2002). Then, it constructs an ontology to consolidate the domain knowledge (Corcho, López, \& Gómez-Pérez, 2003). Finally, the ontological view is used to construct a decision tool supporting the establishment of BPC (Lim, Stolterman, \& Tenenberg, 2008).

Aligning the tenets of design science, each research stage includes two equally important activities: build and evaluate (Hevner et al., 2004; March \& Smith, 1995). The build activities focus on developing artefacts in order to address the research questions, which consist of the scoping knowledge sources, conceptual model, ontology, and decision tool. The evaluate activities then assess the artefacts to provide evidence that they are useful and achieve the stated purposes (Venable, Pries-Heje, \& Baskerville, 2012). Apart from the first stage, scoping and synthesising knowledge sources in order to feed other research activities and thus does not need a separate evaluation, artefacts from the three other stages are carefully evaluated. That is, the conceptual model is assessed through a case study approach (Yin, 2013a). The ontology is evaluated by triangulation. The decision tool is evaluated by experiments (Montgomery, 2012) and focus groups (Tremblay, Hevner, \& Berndt, 2010). Together, the iterations of build and evaluate activities constitute the research process of the dissertation.

Rigour and relevance are critically important in the current design science study. We follow guidelines for ensuring rigour and relevance in design science research suggested by Hevner et al. (2004). In particular, rigour has been managed by building the knowledge base from individual knowledge sources. Further, all the build and evaluate activities of the research process are based on rigorous research techniques. The build activities follow the techniques suggested by Okoli (2015), Webster and Watson (2002), Corcho et al. (2003), and Lim et al. (2008). The evaluations use the empirical techniques suggested by Yin (2013a) for case study evaluation, Montgomery (2012) for experimentation evaluation, and Tremblay et al. (2010) for focus group evaluation. Relevance has been 
realised by addressing the current organisational problem: business process crowdsourcing. Relevance is also achieved by evaluating the outcome artefacts using empirical data collected through case study, experiments and focus groups.

In summary, the research design adopts the guidance for design science research defined by Hevner et al. (2004) and comprises four main stages: scoping knowledge sources, conceptual model, ontology, and decision tool. Each stage includes both the build and evaluate activities, which together constitute the iterative research process. An exception is the first stage, which scopes the knowledge sources for feeding other activities and thus does not need a separate evaluation. These stages are based on rigorous research techniques and relevant data collected from the practical environments.

\subsection{Research Significance}

Positioned within the crowdsourcing field and design science paradigm, the significance of this study concerns both academics and practitioners. From an academic point of view, this research brings the business process lens to crowdsourcing research, which possibly evolves crowdsourcing from one-off processes to repeatable organisational business processes. Just as the movement of business process outsourcing (PBO) contributed to advance the outsourcing field, BPC is a necessary development for moving the crowdsourcing field forward. Further, this development addresses the ad hoc nature of crowdsourcing processes (Thuan et al., 2015b; Zhao \& Zhu, 2014) and responds to the calls for developing an integrated crowdsourcing process (Djelassi \& Decoopman, 2013; Khazankin, Satzger, \& Dustdar, 2012a; Lüttgens, Pollok, Antons, \& Piller, 2014).

In the design science paradigm, the contribution of this research is a set of artefacts establishing BPC: a conceptual model, an ontology, and a decision tool. In particular, the model conceptualises BPC and its building blocks. The ontology consolidates the domain knowledge through defining the concepts, hierarchical relationships and decision-making relationships. Together, the model and ontology fulfils the gap of "little attention to the ontological and conceptual foundations on how to engineer the entire [crowdsourcing] process" (Hosseini, Phalp, Taylor, \& Ali, 2014, p. 1). The decision tool, operationalising the ontology, supports making informed BPC decisions. Therefore, it expands early 
efforts in developing decision support in the domain (Geiger \& Schader, 2014; Prokesch $\&$ Wohlenberg, 2014). Overall, the study contributes a set of design science artefacts for establishing crowdsourcing as an organisational business process.

Another contribution of the study is the empirical evidence that shows how the proposed artefacts work. The empirical results, derived from case studies of two crowdsourcing projects, a triangulation comparing the ontology with a version generated by software, experiments with 190 participants, and two focus groups with ten participants, suggest that the proposed artefacts can be used to effectively support BPC establishment. These empirical results complement our theoretical efforts conceptualising BPC, and other theoretical efforts seeking to consolidate the crowdsourcing process (Amrollahi, 2015; Geiger \& Schader, 2014; Hetmank, 2013).

From a practical point of view, this study provides several means for organisations to establish crowdsourcing in their business processes, including the conceptual model, ontology, and decision tool. The conceptual model and ontology guide how to plan, analyse, and design BPC, while the tool supports business managers and process designers making decisions on the establishment of BPC. We note that the proposed artefacts can be used as individual means or as a set of artefacts supporting BPC. These supports enable organisations to take advantage of crowdsourcing by integrating the strategy into their business processes (Lopez, Vukovic, \& Laredo, 2010; Satzger, Psaier, Schall, \& Dustdar, 2012; Tranquillini et al., 2015).

\subsection{Dissertation Structure}

This chapter has introduced the concept of business process crowdsourcing, and defined the research problem, research objectives, and research questions. It also presented the research methodology and possible contributions. The remaining chapters of this dissertation are structured as follows.

\section{Chapter 2 Literature Overview}

This chapter reviews existing research in crowdsourcing. It identifies the main concepts in the crowdsourcing field and reviews research into crowdsourcing processes. It also 
presents a framework for data collection and analysis. We named this chapter as 'Literature Overview' to distinguish it from the scoping literature review in Chapter 4. Chapter 3 Research Design

This chapter presents the design science paradigm, which has been adopted in the study. It also reviews several design science methods. This review suggests a need for design methods that can be specifically applied to areas with unstructured knowledge, like crowdsourcing. It then discusses the four-stage research process guiding the study.

\section{Chapter 4 The Four Research Stages of Build and Evaluate}

This chapter presents the design cycles of the study, structured as four research stages: scoping knowledge source, conceptual model, ontology, and decision tool. Each stage consists of two activities: build and evaluate. The activities and their results are presented.

\section{Chapter 5 Integrated Results}

This chapter discusses the results from an integrated view, which highlights the interrelation of the research stages. It further consolidates the findings in order to answer the research questions.

\section{Chapter 6 Discussion}

This chapter discusses the contributions of the research. It also discusses the design products and design process of the research that contribute to the knowledge base. Contributions to practice are also discussed, followed by the limitations of the research.

\section{Chapter 7 Conclusion and Future Work}

This chapter summarises the research results and main conclusions. Future research opportunities are outlined.

\subsection{List of Publications}

Several parts of the dissertation have been presented in international journals and peer reviewed conferences, according to the list of publications below. 
Although the publications indicate the collaboration between the author and the supervision team and between the author and the co-authors, I note that the work I have presented here is my own. Nevertheless, the personal pronoun 'we' has been used in the dissertation in accordance with standard scientific protocol.

\section{Journal Publications}

1. Thuan, N. H., Antunes, P., \& Johnstone, D. (2016). Factors Influencing the Decision to Crowdsource: a Systematic Literature Review. Information Systems Frontiers, 18(1), 47-68. [ABDC Rank A; Impact Factor 1.077].

2. Thuan, N. H., Antunes, P., \& Johnstone, D. (2016). A Process Model for Establishing Business Process Crowdsourcing. Australasian Journal of Information Systems (Submission). [ABDC Rank A].

\section{Academic conferences (Peer-reviewed)}

3. Thuan, N. H., Antunes, P., \& Johnstone, D. (2016). Pilot Experiments on a Designed Crowdsourcing Decision Tool. Proceedings of the 20th IEEE International Conference on Computer Supported Cooperative Work in Design (CSCWD2016 Proceedings). pp. 601-606.

4. Thuan, N. H., Antunes, P., Johnstone, D., \& Ha, X. S. (2015). Building an Enterprise Ontology of Business Process Crowdsourcing: A Design Science Approach. The 19th Pacific Asia Conference on Information Systems (PACIS 2015 Proceedings). AISeL, Paper 112.

5. Thuan, N. H., Antunes, P., \& Johnstone, D. (2015). A Design Science Method for Emerging Decision Support Environments. In Australasian Conference on Information Systems (ACIS 2015), Adelaide, Australia. Paper 32.

6. Thuan, N. H., Antunes, P., Johnstone, D., \& Nguyen, H. A. D. (2015). Establishing a Decision Tool for Business Process Crowdsourcing. In Tran Khanh Dang, Roland Wagner, Erich Neuhold, Makoto Takizawa, Josef Küng, \& Nam Thoai (Eds.), the $2^{\text {nd }}$ International Conference on Future Data and Security Engineering (FDSE 2015). LNCS (Vol. 9446, pp. 85-97): Springer, Heidelberg. This paper received a VUWSA Academic Conference Grant 2015. 
7. Thuan, N. H., Antunes, P., \& Johnstone, D. (2014). Toward a Nexus Model Supporting the Establishment of Business Process Crowdsourcing. In Tran Khanh Dang, Roland Wagner, Erich Neuhold, Makoto Takizawa, Josef Küng, \& Nam Thoai (Eds.), the $1^{\text {st }}$ International Conference on Future Data and Security Engineering (FDSE 2014). LNCS (Vol. 8860, pp. 136-150): Springer, Heidelberg. This paper received a VUWSA Postgraduate Research Excellence Award 2014.

8. Thuan, N. H., Antunes, P., Johnstone, D., \& Quang, T. M. N. (2014). An Architecture Utilizing the Crowd for Building an Anti-virus Knowledge Base. In Tran Khanh Dang, Roland Wagner, Erich Neuhold, Makoto Takizawa, Josef Küng, \& Nam Thoai (Eds.), the $1^{\text {st }}$ International Conference on Future Data and Security Engineering (FDSE 2014). LNCS (Vol. 8860, pp. 164-176): Springer, Heidelberg.

9. Thuan, N. H., Antunes, P., \& Johnstone, D. (2013). Factors Influencing the Decision to Crowdsource. In P. Antunes, M. Gerosa, A. Sylvester, J. Vassileva, \& G. J. De Vreede (Eds.), the 19th International Conference on Collaboration and Technology (CRIWG 2013). LNCS (Vol. 8224, pp. 110-125): Springer Berlin Heidelberg. 


\section{LITERATURE OVERVIEW}

This chapter presents a focused literature review that forms a foundation for the dissertation. As crowdsourcing has raised multiple interests, it has been studied in a variety of domains: marketing, management, software engineering, computer science, and information systems. This wide research spectrum enables crowdsourcing to become a young yet rapidly growing research field. Publications in this field cover aspects like decision making, quality control, crowd management, workflow design, system architecture and crowd programming (Hossain, Kauranen, \& Busi, 2015; Zhao \& Zhu, 2014). Consequently, this review draws extensively on varied sources.

The variety of crowdsourcing literature makes the body of knowledge hard to be synthesised. To help achieve a shared structure and understanding of the concept, we propose a layered framework that, based on the well-known pattern in software architecture (Shaw \& Garlan, 1996), provides separation of concerns. Figure 2.1 presents the framework comprising of four layers: conceptualisation, classification, process and establishment. These layers are structured symmetrically (top to bottom) from being more abstract to more concrete, and from overview to focus on the research phenomenon.

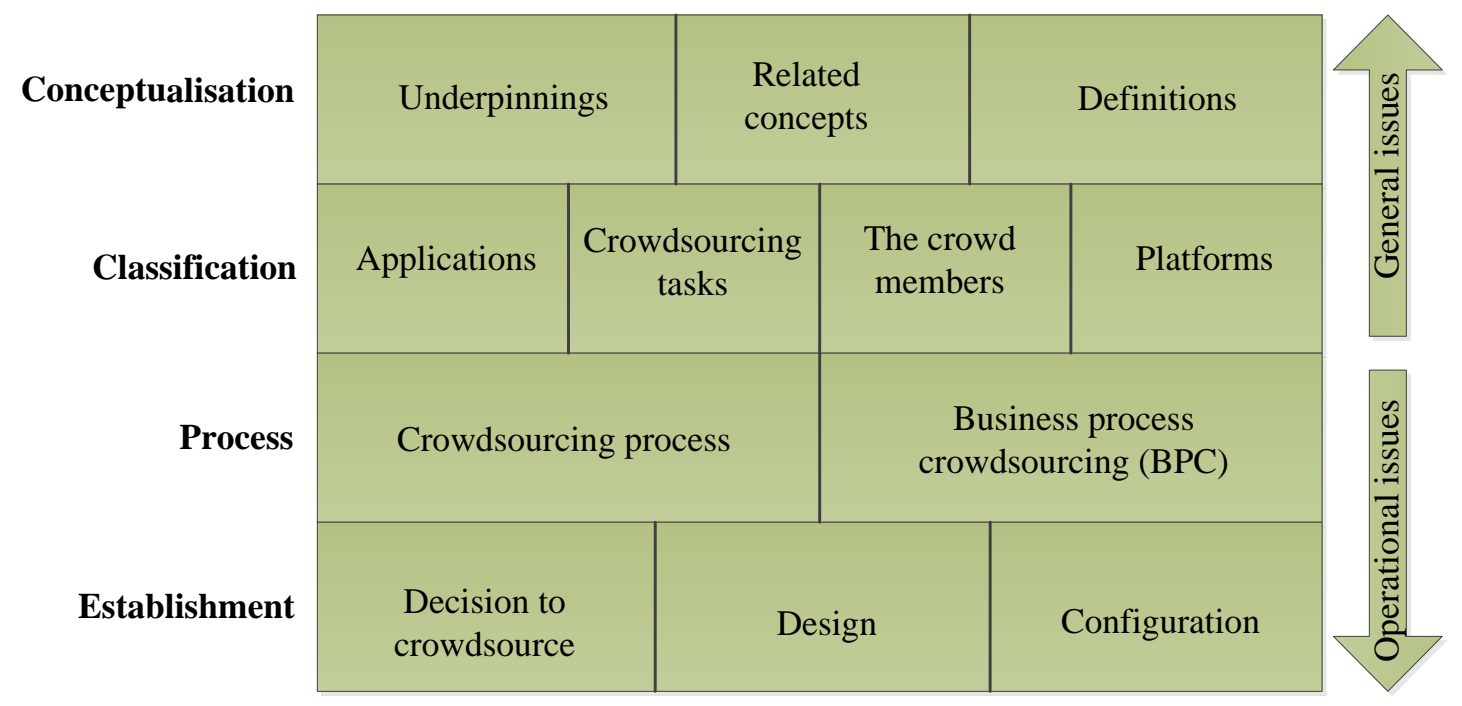

Figure 2.1. Layered framework for the literature review

More precisely, the first layer conceptualises what crowdsourcing is by characterising three major research streams: crowdsourcing underpinnings, related concepts and existing definitions of crowdsourcing. The literature in each stream is reviewed. The second layer 
examines the classifications of crowdsourcing and its related elements. Classifications and taxonomies are focused because they can provide a structured way to organise knowledge in the field (Nickerson, Varshney, \& Muntermann, 2012). Among the different elements classified in the literature, the review highlights the applications, tasks, crowd members and platforms as the most pertinent to this study.

The third layer begins with a review of studies on crowdsourcing processes. The two predominant views, low and high levels of granularity for researching crowdsourcing processes, are reviewed. The focus of this layer then moves to the more specific concept of BPC. The relatively small body of research related to the concept is reviewed for identifying the important roles of BPC and the need for further investigating BPC. The last layer then analyses the three main stages necessary to establish BPC: decision to crowdsource or not, design process, and configuration. These phases serve as an analytical framework for data collection and data analysis of the research.

\subsection{The Concept of Crowdsourcing}

Despite a decade studied, crowdsourcing is difficult to conceptualise. There is considerable confusion surrounding crowdsourcing terminology in terms of concepts and definitions, as crowdsourcing has continuously developed within different research streams. Thus, it is necessary to explain the concept of crowdsourcing. This section commences with a discussion of the basic ideas behind crowdsourcing. It then compares crowdsourcing with other similar concepts. A definition of crowdsourcing used in this dissertation is then provided.

\subsubsection{Main Idea behind Crowdsourcing}

Reliance on the crowd can be traced back to the early 18th century, when the British government decided to provide a cash prize for anyone who could address the problem of precisely calculating ship longitudes (Afuah \& Tucci, 2012). Despite a long history of crowd participation, the concept of crowdsourcing has only really emerged when Howe (2006b) introduces a process utilising the crowd for fulfilling tasks through the Internet. This raises the question why crowdsourcing has become so popular only recently. Investigating this question, researchers propose several underpinnings behind the 
emergence of crowdsourcing (Brabham, 2008a; Palacios, Martinez-Corral, Nisar, \& Grijalvo, 2016; Saxton et al., 2013). By and large, these underpinnings can be grouped into three categories: 1) the crowd, 2) the organisation, and 3) the medium linking the crowd and the organisation.

First, it is now widely accepted that the crowd's wisdom plays a significant role in crowdsourcing (Brabham, 2008a; Hosseini, Shahri, Phalp, Taylor, \& Ali, 2015b; Saxton et al., 2013). The 'wisdom of crowds' was explored by Surowiecki (2004), who claimed that "under the right circumstances, groups are remarkably intelligent, and are often smarter than the smartest people in them" (p. xiii). The right circumstances are defined as four prerequisites: cognitive diversity, independence, decentralisation and aggregation. Under these prerequisites, individual ideas in the crowd are not averaged, but aggregated into final solutions. As a result, the aggregated solutions are better than, or at least equal to, the solutions from individual members in the crowd.

Although the wisdom of the crowd is dominant in explaining the concept of crowdsourcing, some extensions should be added to clarify the current capability of crowdsourcing. Malone et al. (2010) have extended the underpinnings of crowdsourcing by adding the idea of collective intelligence. Different from the 'wisdom of crowds' that is based on the independence of individuals (Surowiecki, 2004), the idea of collective intelligence stresses the collective coordination of individuals (Bonabeau, 2009; Malone et al., 2010). This extension allows crowdsourcing to find solutions in a coordinated way. Another extension is the ability of crowdsourcing to solve not only single puzzles, but complex tasks that may be decomposed into a large number of simpler tasks (Kittur et al., 2013). As a result, the ability of the crowd should be seen from both its individual and collective intelligence and its capability to manage a large number of tasks.

The second category of underpinnings comes from an organisational viewpoint. As the ability of the crowd seems promising, the next question is whether organisations have any demands for using this ability. In fact, they do. The demands for using external agents to perform tasks has been clearly presented in the management literature: outsourcing (Dibbern, Goles, Hirschheim, \& Jayatilaka, 2004; Lacity, Solomon, Yan, \& Willcocks, 2011), open sourcing (Ågerfalk \& Fitzgerald, 2008), and open innovation (Chesbrough, 2013; Seltzer \& Mahmoudi, 2013). With outsourcing, organisations have a long history 
of using contracted resources outside their boundaries. Recently, open sourcing and open innovation have further blurred the organisational boundaries for seeking ideas and innovation beyond the traditional organisational boundaries.

The demands for external sourcing explains the reason why organisations have largely been attracted to crowdsourcing. By adopting crowdsourcing, organisations may get benefits similar to outsourcing, such as cost saving and access to outside capabilities (Rouse, 2010; Saxton et al., 2013), or even more, such as customer involvement and flexible, on-demand labour. These benefits increase organisational demands for crowdsourcing. It is important to note that although organisational demands to use external resources of crowdsourcing are similar to outsourcing and open innovation, these concepts are distinctive because of other characteristics, as discussed in the next section.

Given the aforementioned underpinnings, the term 'crowdsourcing' can be etymologically analysed as a combination of two words: crowd and sourcing. However, the fact that these underpinnings have existed long before the recent emergence of crowdsourcing reveals that another underpinning is needed to enable crowdsourcing. Most of the crowdsourcing literature agrees on the role of the Internet, and in particular the recent dominance of Web 2.0 (Brabham, 2013; Saxton et al., 2013; Zhao \& Zhu, 2014). Web 2.0 has changed the nature of online interaction, where individuals are no longer passive receivers but active contributors (Andriole, 2010; Brabham, 2013; OReilly, 2007). Brabham (2013) notes that Web 2.0 fastens a voluntary participatory culture onto a global, virtual environment, where Internet users seem keen to contribute their ideas, knowledge, skills, and labour. Such contributions are valuable resources for work.

From an organisational perspective, Web 2.0 empowers the open call that allows any given interested participants can participate in, which is a distinctive characteristic of crowdsourcing. Because of its millions of users, Web 2.0 extends the scope of the open calls through providing a valuable medium for approaching innumerable anonymous audiences (Saxton et al., 2013). It has also eased users to participate in a variety of Internet activities with fewer barriers, e.g. regarding time and space (Brabham, 2013). As a result, it extends the reach and the scope of the crowdsourcing open calls. 
This review has shown that, the combination of the crowd, Web 2.0, and organisational demands, can explain the emergence of crowdsourcing. Given these underpinnings, the IS discipline, which is concerned with people, technologies, and organisations (Bacon \& Fitzgerald, 2001), has crowdsourcing as a focus point. This focus point also comes from a strength of the IS research, which draws upon reference disciplines to build its own knowledge base (Baskerville \& Myers, 2002). This is exactly the need for the field of crowdsourcing, as a large part of research into crowdsourcing is not very well delimited. All in all, we believe that IS research like the current research can make significant contributions to the crowdsourcing field.

The review has also shown that no single underpinning can enable crowdsourcing per se, but rather the combination of the three underpinnings supports the emergence of crowdsourcing. This combination distinguishes crowdsourcing from other concepts, being presented in the next section.

\subsubsection{Related Concepts}

In another stream of research attempting to clarify the concept of crowdsourcing, many researchers compare and contrast this notion with similar concepts, such as open innovation, outsourcing, open source, and peer production. This section reviews this research stream and discusses crowdsourcing by comparing its similarities and differences with the related concepts.

Among the competing concepts, one often discussed in relation to crowdsourcing is open innovation. Crowdsourcing and open innovation share a common basis where organisations harvest knowledge and expertise from the outside, the opposite of closed innovation. As a result, some researchers suggest that crowdsourcing belongs to or is a technique of open innovation (Marjanovic, Fry, \& Chataway, 2012; Seltzer \& Mahmoudi, 2013). However, other researchers argue that these two concepts are different, at least in two important points. The first one is that open innovation only focuses on innovation processes, while crowdsourcing can be used for various types of tasks (Nakatsu, Grossman, \& Iacovou, 2014; Schenk \& Guittard, 2011). Second, organisations interact mainly with other firms and their customers in open innovation, but rely on anonymous members of the crowd in crowdsourcing activities (Schenk \& Guittard, 2009; Zhao \& Zhu, 2014). 
Outsourcing is another concept closely related to crowdsourcing. As noted in the previous section, the two concepts are similar on the organisational demands for external agents. As a result, pioneering researchers considered crowdsourcing as a form of outsourcing (Howe, 2006b; Rouse, 2010; Whitla, 2009). Nevertheless, recent conceptualisations of crowdsourcing clearly identify the differences between these two concepts. One major difference is who performs the activities. Actors performing tasks in crowdsourcing are informal members of the crowd, while in outsourcing they are mainly established supplier firms (Ågerfalk, Fitzgerald, \& Stol, 2015). Another difference lies in how to manage these actors. Compared to the official contracts with certain preselected suppliers used in outsourcing, crowdsourcing uses an open call where any member in the crowd can participate in the project (Zhao \& Zhu, 2014). Finally, financial incentives are the main motivation for task performers in outsourcing, whereas crowdsourcing can be based on both intrinsic incentive, e.g. personal enjoyment and hobby, and extrinsic incentives, e.g. money (Hossain, 2012; Kaufmann, Schulze, \& Veit, 2011).

The literature also distinguishes crowdsourcing from open source, although the two concepts are based on resources from the community to accomplish tasks. There are two key aspects distinguishing them: management and engagement. In crowdsourcing, activities are managed by the organisations, whereas in open source these activities are self-managed and driven by the community (Brabham, 2013). Regarding to how the community is engaged to perform the activities, crowdsourcing outcomes can be achieved either independently or collaboratively (Geiger, Seedorf, Schulze, Nickerson, \& Schader, 2011; Zhao \& Zhu, 2014), but outcomes from open source are achieved mainly through collaboration. The motivation of the community is another difference between these two concepts. Most of the time, members in open source communities perform tasks based on intrinsic motivation (Brabham, 2008a), whereas both intrinsic and extrinsic motivations can be found in crowdsourcing (Kaufmann et al., 2011). Furthermore, unlike open source, crowdsourcing campaigns clearly have intellectual property rights and are not restricted to software development (W. Wu, Tsai, \& Li, 2013).

A few researchers equate crowdsourcing to a form of peer production (Mason \& Watts, 2009; F. Wu, Wilkinson, \& Huberman, 2009). These researchers believe that peer production sites, like Youtube, can be seen as crowdsourcing because contents on these sites are created by mass individuals in the crowd. However, other researchers argue that 
crowdsourcing is completely different from peer production. Estellés-Arolas and González-Ladrón-de-Guevara (2012) suggest that crowdsourcing tasks require clear objectives, and thus Youtube, where an individual can upload any video, is not crowdsourcing. In addition, peer production mainly depends on particular communities (Haythornthwaite, 2009; Huberman, Romero, \& Wu, 2009), whereas crowdsourcing relies on anonymous members of the crowd, as previously mentioned.

To summarise the above discussion, this review adapts Malone et al.'s (2010) framework to compare crowdsourcing with the related concepts. This framework includes four questions: what needs to be performed, who is performing the task, why people do this, and how the task is being done. An additional question about controlling intellectual property (IP) is added for clarifying the locus of control on the outcomes. By answering the five questions (five rows), the main differences between crowdsourcing and the other concepts are presented in Table 2.1. This table reflects that crowdsourcing is a distinctive notion, leading us to investigate the concept per se.

Table 2.1. Main differences between crowdsourcing and related concepts

\begin{tabular}{|c|c|c|c|c|c|}
\hline & $\begin{array}{c}\text { Open } \\
\text { innovation }\end{array}$ & $\begin{array}{c}\text { Out } \\
\text { sourcing }\end{array}$ & $\begin{array}{c}\text { Open } \\
\text { source }\end{array}$ & $\begin{array}{c}\text { Peer } \\
\text { production }\end{array}$ & Crowdsourcing \\
\hline Tasks & $\begin{array}{l}\text { - Only } \\
\text { innovation }\end{array}$ & & - Software & $\begin{array}{l}\text { - Undefined } \\
\text { tasks }\end{array}$ & $\begin{array}{l}\text { - Varied types of } \\
\text { tasks } \\
\text { - Predefined tasks }\end{array}$ \\
\hline Workforce & \begin{tabular}{|l} 
- Other firms \\
\& customers \\
\end{tabular} & $\begin{array}{l}\text { - Supplier } \\
\text { firms }\end{array}$ & $\begin{array}{l}\text { - Software } \\
\text { community } \\
\end{array}$ & $\begin{array}{l}\text { - Certain } \\
\text { community }\end{array}$ & $\begin{array}{l}\text { - Members of the } \\
\text { crowd }\end{array}$ \\
\hline $\begin{array}{l}\text { Participant } \\
\text { Motivation }\end{array}$ & & 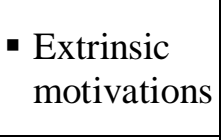 & $\begin{array}{l}\text { - Intrinsic } \\
\text { motivations }\end{array}$ & & $\begin{array}{l}\text { - Intrinsic \& } \\
\text { extrinsic } \\
\text { motivations } \\
\end{array}$ \\
\hline $\begin{array}{l}\text { Nature of } \\
\text { management } \\
\text { and } \\
\text { engagement }\end{array}$ & & $\begin{array}{l}\text { - Official } \\
\text { contracts }\end{array}$ & $\begin{array}{l}\text { - Workflows } \\
\text { and quality } \\
\text { control } \\
\text { managed by } \\
\text { community } \\
\text { - Collaborative }\end{array}$ & - Collaborative & $\begin{array}{l}\text { - Open call } \\
\text { - Without official } \\
\text { contract } \\
\text { - Workflows and } \\
\text { quality control } \\
\text { mainly managed } \\
\text { by the } \\
\text { organisations } \\
\text { - Collaborative \& } \\
\text { independent }\end{array}$ \\
\hline Control on IP & & & - IP open & & - IP protected \\
\hline
\end{tabular}




\subsubsection{Crowdsourcing Definition}

From the above discussion on the different concepts related to crowdsourcing, it is not surprising that researchers have defined the crowdsourcing concept differently. This section presents a brief history of crowdsourcing definitions in order to understand the concept evolution, and ultimately to form a definition for use in this dissertation.

Until now, crowdsourcing has a short history of one decade. The phenomenon began to appear in 2006 after Howe (2006b) coined this term when he observed several websites utilising Internet users to perform certain activities. It is interesting to note that Howe's (2006b) article has appeared in Wired Magazine - a news media, which indicates that crowdsourcing is a concept spreading from practice to academia. In the article, crowdsourcing was described as the act of organisations through the form of an open call in order to "tap the latent talent of the crowd" (Howe, 2006b, p. 2). In the same year, he proposed the first definition of crowdsourcing.

"Simply defined, crowdsourcing represents the act of a company or institution taking a function once performed by employees and outsourcing it to an undefined (and generally large) network of people in the form of an open call. This can take the form of peerproduction (when the job is performed collaboratively), but is also often undertaken by sole individuals. The crucial prerequisite is the use of the open call format and the large network of potential laborers" (Howe, 2006a).

Up to now, this definition is among the ones most cited in the field due to its exploratory nature and simplicity. It is worth noting two interesting points from this definition. First, it views organisations as the main caller who operationalise crowdsourcing, which is completely aligned with the promotion of crowdsourcing for organisations in the dissertation. Second, in this definition, crowdsourcing is a sourcing strategy and is an extension of outsourcing.

After 2006, researchers started to explore crowdsourcing and soon published several alternative definitions. Since then, crowdsourcing definitions have evolved over time. Figure 2.2 summarises the evolution of crowdsourcing definitions during the last decade. 


\begin{tabular}{|c|c|c|c|c|}
\hline $\begin{array}{l}\text { The first } \\
\text { definition }\end{array}$ & $\begin{array}{c}\text { Academic } \\
\text { definitions, with } \\
\text { two main streams }\end{array}$ & $\begin{array}{c}\text { Additional features } \\
\text { to the definition }\end{array}$ & $\begin{array}{c}\text { Integrated } \\
\text { definition, which is } \\
\text { widely accepted }\end{array}$ & $\begin{array}{l}\text { Adapted the } \\
\text { integrated definition } \\
\text { to research and } \\
\text { application contexts }\end{array}$ \\
\hline $\begin{array}{l}\text { + Ways to tap } \\
\text { the latent } \\
\text { talent of the } \\
\text { crowd } \\
+ \text { An extension } \\
\text { of outsourcing } \\
\text { (Howe, 2006a; } \\
\text { Howe, 2006b) }\end{array}$ & \begin{tabular}{||l} 
+ Outsource task to \\
the crowd, which is \\
now an alternative \\
workforce (Ågerfalk \\
\& Fitzgerald, 2008; \\
Howe, 2008; \\
Whitla, 2009; Yang \\
et al. 2008) \\
+ A model for \\
problem solving \\
(Brabham, 2008a; \\
Brabham, 2008b; \\
DiPalantino \& \\
Vojnovic, 2009; \\
Vukovic, 2009)
\end{tabular} & $\begin{array}{l}\text { + Incentive } \\
\text { mechanism (Alonso } \\
\text { \& Baeza-Yates, 2011; } \\
\text { Kaufmann et al., } \\
2011 ; \text { Kazai, 2010) } \\
+ \text { Nature of the } \\
\text { crowd (Buecheler et } \\
\text { al., 2010; Doan et } \\
\text { al., 2011) } \\
+ \text { Nature of tasks } \\
\text { (Ipeirotis et al., } \\
\text { 2010) } \\
+ \text { Business process } \\
\text { crowdsourcing } \\
\text { (Vecchia \& } \\
\text { Cisternino, 2010) }\end{array}$ & $\begin{array}{l}\text { + Integrated } \\
\text { definition with the } \\
\text { following features } \\
\text { (Estellés-Arolas \& } \\
\text { González-Ladrón-de- } \\
\text { Guevara, 2012): } \\
\text { 1. a defined crowd; } \\
\text { 2. a delineated task; } \\
\text { 3. a clear } \\
\text { recompense for the } \\
\text { crowd; } \\
\text { 4. the identified } \\
\text { crowdsourcer; } \\
\text { 5. defined benefits } \\
\text { for the } \\
\text { crowdsourcer; } \\
6 . \text { an online process; } \\
\text { 7. the open call; } \\
\text { 8. internet usage }\end{array}$ & $\begin{array}{l}\text { + Crowdsourcing } \\
\text { innovation } \\
\text { (Majchrzak \& } \\
\text { Malhotra, 2013; Xu } \\
\text { et al., 2015) } \\
\text { + Software (Stol \& } \\
\text { Fitzgerald, 2014; } \\
\text { Zogaj et al., 2014) } \\
\text { + Information } \\
\text { Systems (Pedersen } \\
\text { et al., 2013; Zhao \& } \\
\text { Zhu, 2014) }\end{array}$ \\
\hline
\end{tabular}

Figure 2.2. Evolution of crowdsourcing definitions

After Howe's (2006a) definition, several academic definitions of the concept were published between 2008 and 2009. Extending Howe's (2006a) view, some researchers conceptualised crowdsourcing as a sourcing model where the task performers were the crowd. These researchers further defined who the crowd was and positioned it as a workforce alternative to internal employees and outsourcing agents (Ågerfalk \& Fitzgerald, 2008; Howe, 2008; Whitla, 2009; Yang, Adamic, \& Ackerman, 2008). At the same time, a parallel approach focused on the intelligence capabilities of crowdsourcing. Researchers in this approach defined crowdsourcing as a problem solving model, where the crowd contributes not only with labour but also with creativity (Brabham, 2008a, 2008b; DiPalantino \& Vojnovic, 2009; Vukovic, 2009). As a pioneer researcher in this stream, Brabham (2008b) summarised the notion of crowdsourcing as "a process, a model, for distributed problem solving through the Web" (p. 1). The term 'problem' in Brabham's definition should be understood in a broad sense, including not only R\&D problems but also design and innovation. 
During 2010-2011, there was a boom of crowdsourcing definitions, aligning to a diverse set of practices and an increasing number of research interests in the field. At this stage, researchers adopted different theoretical bases and models to investigate several aspects of crowdsourcing. Depending on the research foci, the related features were depicted and added to crowdsourcing definitions, including the nature of the crowd (Buecheler, Sieg, Füchslin, \& Pfeifer, 2010; Doan, Ramakrishnan, \& Halevy, 2011), the nature of tasks (Ipeirotis, Provost, \& Wang, 2010), and incentive mechanisms (Alonso \& Baeza-Yates, 2011; Kaufmann et al., 2011; G. Kazai, 2010). These definitions, on the one hand, contribute to clarifying several features of the concept. On the other hand, definitions with too many additional features suffer from diversity and sometimes conflict with each other, which makes crowdsourcing hard to comprehend.

Addressing this problem, in 2012, Estellés-Arolas and González-Ladrón-de-Guevara (2012) aimed at establishing an integrated definition of crowdsourcing. Driving their research was the goal to conceptualise 'any given crowdsourcing activity'. The authors reviewed the diverse definitions extracted from literature. A selection of 209 crowdsourcing articles was examined and 40 of them presenting original definitions of crowdsourcing were analysed. The results suggest eight common characteristics of crowdsourcing: a clearly defined crowd, a task with a clear goal, a clear recompense for the crowd, an identified crowdsourcer (or caller), defined compensation for the crowdsourcer, an online process, an open call, and Internet usage. The authors then integrate these characteristics into a single comprehensive definition.

"Crowdsourcing is a type of participative online activity in which an individual, an institution, a non-profit organisation, or company proposes to a group of individuals of varying knowledge, heterogeneity, and number, via a flexible open call, the voluntary undertaking of a task. The undertaking of the task, of variable complexity and modularity, and in which the crowd should participate bringing their work, money, knowledge and/or experience, always entails mutual benefit. The user will receive the satisfaction of a given type of need, be it economic, social recognition, self-esteem, or the development of individual skills, while the crowdsourcer will obtain and utilize to their advantage what the user has brought to the venture, whose form will depend on the type of activity undertaken” (Estellés-Arolas \& González-Ladrón-de-Guevara, 2012, p. 197). 
Due to its comprehensiveness, this definition has been widely accepted and frequently referred to. Yet, the definition is wordy and thus complex, which decreases its practical use. As a result, many recent studies have implicitly or explicitly adapted the aforementioned eight characteristics by simplifying and adjusting them to their own research and application contexts. For instance, crowdsourcing software emphasises the software tasks being crowdsourced (Stol \& Fitzgerald, 2014) and the intermediated platforms (Zogaj, Bretschneider, \& Leimeister, 2014); crowdsourcing innovation focuses on the innovative ability of the crowd (Majchrzak \& Malhotra, 2013; Xu, RibeiroSoriano, \& Gonzalez-Garcia, 2015). These adaptions show that there is no unique universal definition of crowdsourcing appropriate for all applications and research contexts, though Estellés-Arolas and González-Ladrón-de-Guevara's definition may form a basic understanding.

Aligning to the most recent trend, this dissertation simplifies and adapts the definition by Estellés-Arolas and González-Ladrón-de-Guevara (2012) to the organisational context. We use the following definition.

Crowdsourcing is an online strategy in which an organisation proposes defined task(s) to the members of the crowd via a flexible open call. By undertaking the task(s), the members contribute their work, knowledge, skills and/or experience and receive rewards, including economic rewards, social recognition, selfesteem, or the development of individual skills. The organisation will obtain contributions from the crowd and will utilise the results to meet business goals.

To sum up, this review has shown the conceptualisation of crowdsourcing, through three facets. The first facet has shown the three main pillars of crowdsourcing: the organisational demands for external sourcing; the ability of the crowd; and the intermediary Web 2.0. These pillars together enable crowdsourcing. The second facet has compared and differentiated crowdsourcing with related concepts, like open innovation, outsourcing, open sourcing and peer production. It emphasises the distinctive characteristics of crowdsourcing. The final facet has shown a brief evolution in crowdsourcing definitions. It then proposes the definition that to be used in this dissertation. From these facets, it is worth noting that although a few referencing theories have been applied to explain crowdsourcing, like the wisdom of the crowd, open 
innovation, and outsourcing practices, the distinctive characteristics of crowdsourcing state that these theories are not predominant in the phenomenon. Consequently, crowdsourcing is a concept per se that needs its own structures and establishment.

The next section discusses key themes on crowdsourcing structures and classifications.

\subsection{Crowdsourcing Classifications, Taxonomies, and Typologies}

As classifications and taxonomies are useful to structure knowledge in the IS discipline (Nickerson et al., 2012), an extensive body of crowdsourcing literature is devoted to crowdsourcing classifications, taxonomies, and typologies. Although they contribute to structuring the domain, each of them focuses on different crowdsourcing elements. This section considers the popular classified elements: applications, tasks, members and platforms, which are essential for exploring crowdsourcing processes. In particular, the review aims to answer the following questions: What are the crowdsourcing applications? Which types of tasks can be crowdsourced? Who will perform these tasks? And where can these tasks be performed?

Before proceeding, it is useful to clarify terminologies of classification, as a variety of them have been used in the literature, including classification, taxonomy, and typology. The term classification is used to refer to both a product and a process of classifying objects according to a particular system (Fettke \& Loos, 2003). Taxonomy and typology are two forms of classification that usually deploy multi-dimensions to classify objects into categories. Some researchers further distinguish taxonomies as empirical classifications and typologies as conceptual classifications (Bailey, 1994). However, other researchers suggest using classification, taxonomy, and typology interchangeably (Gregor, 2006; Nickerson et al., 2012). We follow this suggestion as we observe that the crowdsourcing literature commonly refers to the three terms in an interchangeable way. Consequently, the review uses these terminologies more or less synonymously.

\subsubsection{Applications}

Crowdsourcing can be utilised for different applications. Howe (2006b) discusses the crowdsourcing concept through several applications in solving real business problems, 
including iStockphoto for image exchange, InnoCentive for problem solving, and AMT for micro tasks. Not only limited to business applications, crowdsourcing can also be applied to scientific research (Mason \& Suri, 2012), urban planning (Brabham, 2012b), public health (Brabham, Ribisl, Kirchner, \& Bernhardt, 2014), and cultural heritage (Ridge, 2014). Given the variety of crowdsourcing applications, their classifications are necessary for understanding the potential utility of crowdsourcing. Addressing this necessity, several application classifications have been proposed in the literature, which are now reviewed in detail. The review moves from simple classifications, defining for specific areas, to more inclusive typologies at the level of work practice.

Some studies, maybe for simplification, classify crowdsourcing applications specifically for one single area. For instance, Whitla (2009), focusing on marketing-related areas, classifies crowdsourcing applications into three function categories, namely product development, advertising and promotion, and marketing research. Gomes et al. (2012) propose a crowdsourcing taxonomy with a focus on the context of musical productions. Based on what crowdsourcing can be utilised for, the taxonomy identifies six types of applications: music co-creation, decision support, crowdsourced music collection and management, promoting music information, market place, and crowd funding.

Adopting a broader approach, other studies propose a number of application typologies that can be used in multiple domains. Kleeman et al. (2008) explored start-up crowdsourcing applications, and typologically grouped them according to their functions. As a result, seven application types are defined, namely product development and configuration, product design, competitive bids, permanent open calls, community reporting, product rating, and customer-to-customer support. This approach is also employed by Brabham in his recent book, Crowdsourcing (2013). He, surveying crowdsourcing cases, conceptualises them into four different functions, including knowledge discovery and management, broadcast search, peer-vetted creative production, and human intelligence tasks. Other typologies which follow a similar approach are mentioned in the literature (Man-Ching, King, \& Kwong-Sak, 2011).

The studies reviewed so far have a common point. They suggest that function is a main dimension to classify crowdsourcing applications. Agreeing with this suggestion, we note, however, that functions alone seem not enough, since a context, where 
crowdsourcing is applied, plays an equally significant role. Chandler and Kapelner (2013), who conducted an experiment on AMT, find that if the context is explained, more workers are willing to participate in the crowdsourcing application. In addition, whether it is a business or non-business context strongly influences the application operation because the context directly links to incentives that may be required to attract people to participate in the crowdsourcing applications (Rosen, 2011).

Given the importance of contexts in characterising crowdsourcing applications, two dimensions: function and context together are likely more appropriate to classify applications. This appropriateness is supported by Zhao and Zhu (2014), who broadly reviewed crowdsourcing applications. By deductively analysing 126 applications, they propose a typology based on the two dimensions of function and context. In the first dimension, these authors group functions into four categories: design and development, test and evaluation, idea and consultant, and others. In the second dimension, two categories of contexts are suggested: business and non-business. A business context consists of for-profit organisations, while non-business includes non-profit organisations and institutions (Zhao \& Zhu, 2014). Although this dimension considers contexts at an organisational level, we suggest the level of application is more precise for this dimension. The reason is that one organisation may have both business and non-business applications, such as Amazon owning AMT for profit and QuestVille for non-profit (Saxton et al., 2013). In this case, the context dimension does not associate with the organisation but with its applications. Therefore, this dissertation adopts the typology proposed by Zhao and Zhu (2014), yet considers both the function and context dimensions from the viewpoint of crowdsourcing applications (Table 2.2).

Table 2.2. Typology of crowdsourcing applications (Zhao \& Zhu, 2014)

\begin{tabular}{|l|l|l|l|l|}
\hline \multicolumn{1}{|c|}{$\begin{array}{c}\text { Function } \\
\text { Context }\end{array}$} & \multicolumn{1}{c|}{$\begin{array}{c}\text { Design \& } \\
\text { Development }\end{array}$} & $\begin{array}{c}\text { Idea \& } \\
\text { Consultant }\end{array}$ & $\begin{array}{c}\text { Test \& } \\
\text { Evaluation }\end{array}$ & \multicolumn{1}{|c|}{ Other } \\
\hline Business & - Threadless & - MyStarbucks & - Crowdspirit & - AMT \\
- IStockphoto & $\begin{array}{c}\text { Idea } \\
\text { - InnoCentive }\end{array}$ & & \\
\hline Non-business & $\begin{array}{c}\text { - NextStopDesign } \\
\text { (Brabham, 2012b) }\end{array}$ & - QuestVille & - UTest & - Wikipedia \\
\hline
\end{tabular}




\subsubsection{Tasks}

Tasks are basic elements of a crowdsourcing application. Organisations define tasks and send them to members in the crowd, who will perform these tasks (Howe, 2006b; Whitla, 2009; Zhao \& Zhu, 2014). Several studies have suggested clearly identifying task characteristics before crowdsourcing, which helps to determine the appropriate approach for a particular task (Malone et al., 2010; Nakatsu et al., 2014; Rosen, 2011). Several taxonomies characterising tasks have been proposed in the literature.

There are two main views on building task taxonomies regarding whether tasks should be examined in related with other elements or by its own nature. On the one hand, a number of published taxonomies are based not only on task properties, but also on "key questions [elements] associated with a single task" (Malone et al., 2010, p. 22). Rouse (2010) provides one the of the earliest taxonomies, structured around three dimensions: nature of the task, distribution of benefits, and forms of motivation. In a similar vein, Malone et al. (2010) propose a multi-dimensional classification after analysing 250 instances of crowdsourcing. They base their classification on four basic questions, structured around tasks: what is being crowdsourced, who is performing the task, why would people do this, and how is the task to be done. In these cases, the developed taxonomies suggest multiple dimensions for classification, with task as a central dimension.

On the other hand, another group of published taxonomies classifies tasks by their own nature. By examining the task characteristics in practice, Schenk and Guittard (2009, 2011) suggest two dimensions to classify crowdsourcing tasks. The first dimension classifies tasks as simple, complex or creative. Simple tasks are jobs that can be accomplished with generic skills. Complex tasks require expertise and problem solving skills. Creative tasks relate to individual creativity, such as logo design. The second dimension distinguishes between the integrative and selective nature of tasks (Schenk \& Guittard, 2011). Other taxonomies in this group can also be found in work by Nakatsu et al. (2014).

Given the existing taxonomies, a critical question is which one will be used in this dissertation. To answer this question, the dissertation adopts Nickerson et al.'s (2012) recommendation that the most crucial criterion to evaluate a taxonomy and its dimensions is usefulness. Thus, choosing dimensions for task classification in the dissertation should 
be based on their usefulness for the dissertation focus. That is, the establishment of BPC, consisting of three stages: the decision to crowdsource, process design and configuration, will be discussed in Section 2.4. In the first stage, Zhao and Zhu (2014) suggest examining the complexity of tasks before making this decision. In the remaining stages, we believe that whether tasks are achieved individually or competitively, influences the crowdsourcing design and operation, because it directly affects how the crowd approaches the tasks (Hetmank, 2013).

Consequently, this study employs the two dimensions proposed by Schenk and Guittard (2011): task complexity (simple, complex and creative) and the difference between integration and selection based crowdsourcing. In task complexity, it is worth noting that most complex tasks also require a certain level of creativity, while creative tasks normally seek to find solutions for complex problems. Consequently, the difference between complex and creative tasks is not large and this study combined them into "skilled" tasks. This is consistent with Brabham (2012b), who views a creative activity, like architectural design, as a problem solving task. In the other dimension, the integration and selection difference can also be referred to as the participation mode, representing how tasks can be performed individually or competitively. Table 2.3 presents examples of different types of crowdsourcing tasks (and their related platforms).

Table 2.3. Examples of crowdsourcing task types

\begin{tabular}{|c|c|c|}
\hline $\begin{array}{l}\text { Participation mode } \\
\text { Complexity }\end{array}$ & $\begin{array}{c}\text { Individual } \\
\text { (Integrative) }\end{array}$ & $\begin{array}{c}\text { Competitive } \\
\text { (Selective) }\end{array}$ \\
\hline Simple & \begin{tabular}{l}
\multicolumn{1}{c}{ Market place } \\
- Simple tasks (AMT \& \\
Taskcn)
\end{tabular} & \begin{tabular}{|l}
\multicolumn{1}{c}{ Simple contest } \\
- Answering simple questions \\
(Ask Ville by Amazon \& \\
Yahoo Answers)
\end{tabular} \\
\hline Skilled & $\begin{array}{l}\text { Collective intelligence } \\
\text { - Writing \& editing } \\
\text { (Wikipedia) } \\
\text { - Writing academic papers } \\
\text { (Tomlinson et al., 2012) }\end{array}$ & $\begin{array}{l}\text { Problem solving contest } \\
\text { - Designing T-shirts } \\
\text { (Threadless) } \\
\text { - Problem solving } \\
\text { (InnoCentive) }\end{array}$ \\
\hline
\end{tabular}




\subsubsection{Members of the Crowd}

Crowd members are actors who accomplish tasks in crowdsourcing applications. There are several studies examining characteristics of crowd members. In general, these studies can be grouped into one of two research directions. The first direction examines the crowd characteristics by exploring its properties, such as who members of the crowd are and where they come from. Studies by Mason and Suri (2012) and Brabham (2011) can be categorised in this direction. Another direction studies the crowd as a whole and assesses its performance (Chandler \& Kapelner, 2013; Stewart, Lubensky, \& Huerta, 2010).

In the first direction, Brabham (2011) changed the popular image of the crowd being amateur. By conducting a survey on iStockphoto and several interviews on Threadless, he finds that members on both of these platforms "seem ill-fitted to the amateur label" (Brabham, 2011, p. 399). Specifically, 47\% of participants on IStockphoto described themselves as professional, while many members on Threadless have previously performed real design activities (Brabham, 2011). The argument that the crowd is not wholly amateur, and thus can be in competition with professionals, is also supported by other studies. Jeppesen and Lakhani (2010), who examined the members on InnoCentive, report that " $65 \%$ of solvers reported holding Ph.D. degrees" (p. 1026). Poetz and Schreier (2012), conducting a case study in the idea contest, find that the crowd can outperform the professionals in certain aspects of idea quality.

Similar to Brabham (2011) in exploring the properties of the crowd, Mason and Suri (2012) focusing on AMT present several aspects of AMT's 'workers'. For instance, there are about 100,000 workers on AMT, who are mainly from USA and India. This crowd has more females than males. These characteristics are consistent with findings from another study of 1,000 workers using the same platform conducted by Paolacci et al. (2010), who further report that the hourly average wage on this platform is $\$ 1.66$. From these observations, three reasons provided by Mason and Suri (2012) to choose AMT for online experimentation can be generalised as the crowd characteristics on AMT: large pool of workers, pool diversity and low cost.

In the other direction, studies investigating performance of the crowd as a whole show that the performance is not as promising as the characteristics presented in the first direction. The fact that not all members of the crowd actively performed tasks was 
analysed by Stewart et al. (2010), building on the participation inequality rule of online community (Nielsen, 2006). By analysing a crowd of 400,000 members in a language translation application, these authors separate members of the crowd into three categories: super contributors $(1 \%)$ who provide the most contributions, contributors $(66 \%)$ who provide the moderate contributions and outliers who rarely contribute (33\%). Further analysing the crowd members, Kazai et al. (2011) find that members may perform tasks dishonestly, randomly, or sloppily. In a similar vein, Vuurens and De Vries (2012) suggest a theoretical typology classifying four types of workers regarding their behaviours: diligent workers, sloppy workers, random spammers, and uniform spammers. From the given discussion, some characteristics of the crowd should be highlighted. On the one hand, the crowd is promising in terms of providing a large, diverse, and low-cost workforce (Mason \& Suri, 2012). It may also include 'self-selected' experts (Brabham, 2011). On the other hand, members of the crowd have different levels of contribution for accomplishing tasks (Stewart et al., 2010; Vuurens \& De Vries, 2012). We note that the reviewed studies mainly identify the crowd characteristics based on individual applications and platforms, which implies that the characteristics of the crowd may be different in varied applications and contexts.

\subsubsection{Platforms}

Platform is another key element of crowdsourcing, which serves as a mediator connecting the organisation and the crowd (Hirth, Hoßfeld, \& Tran-Gia, 2011). Vukovic (2009) describes several functions of a crowdsourcing platform: "issues authentication credentials for requestors and providers when they join the platform, stores details about skill-set, history of completed requests, handles charging and payments, and manages platform misuse" (p. 687). Aiming to utilise crowdsourcing, organisations can choose either to develop their own platforms or to use the available ones provided by a third party. Each approach has its own advantages and disadvantages.

Some examples of organisations developing their own crowdsourcing platforms are Threadless, and MyStarbucksIdea in the business context, and Next Stop Design in the non-business context (Brabham, 2012b). Through self-development, organisations can fully control the application and its functions, such as tracking geographic locations of visitors for research purposes in case of Next Stop Design (Brabham, 2012b). Another 
advantage of this approach is building closer relationships with their own customers, who associate with the platforms. For instance, Threadless uses a self-developed platform to ask customers to design $\mathrm{T}$-shirts, and then sells those $\mathrm{T}$-shirts to the customers (Brabham, 2010). Despite these advantages, this approach requires organisations having experts and experience in developing crowdsourcing platforms, since the platform development may have several complex requirements (Adepetu, Ahmed, Al Abd, Al Zaabi, \& Svetinovic, 2012; Vukovic, 2009).

As an alternative to self-development, organisations can hire existing crowdsourcing platforms built by a third party to deploy their applications. The existing platforms can be further divided into two kinds: specialised platforms, which focus on particular tasks (Hirth et al., 2011; Hoßfeld et al., 2013); and horizontal platforms, which can be utilised for different types of tasks (Kucherbaev et al., 2013). An example of a specialised platform is InnoCentive, utilising the crowd only for problem solving purposes (Malone et al., 2010). Differently, horizontal platforms publish different types of tasks. AMT is a typical horizontal platform, which can help an organisation to do several tasks, including data collection, transcription, and image categorisation. To configure a crowdsourcing application on horizontal platforms like AMT, organisations need to use the provided application programming interface (API) (Ipeirotis et al., 2010). Thus, basic programming skills and platform knowledge are required.

Using existing platforms can save organisations' resources, which would otherwise need to be spent on developing their own new platform. Furthermore, existing platforms already have their own members, who are available for new crowdsourcing applications. For instance, an application developed on AMT can use any number of 100,000 available workers (Mason \& Suri, 2012). However, existing platforms limit crowdsourcing applications to what is supported by the platforms. From the above discussion, it is important to note that both approaches (self-developed and existing platforms) have their own pros and cons, which should be considered when making the decision to build or to hire a crowdsourcing platform. 
In summary, the preceding review identified major classifications in the crowdsourcing literature, including applications, tasks, members, and platforms. A full list of the crowdsourcing classifications is presented in Appendix A. On the one hand, these classifications suggest possible options and features that are available in crowdsourcing, which contributes to initially structure the domain (Iivari, 2007). On the other hand, many of them have focused on specific aspects of crowdsourcing and on specific crowdsourcing contexts. This leads to differences, sometimes conflicting, on the domain structures. For instance, crowdsourcing tasks can be classified differently using four dimensions (Malone et al., 2010), three dimensions (Rouse, 2010), or two dimensions (Schenk \& Guittard, 2011).

We believe that this is symptomatic of a more general issue with the ad hoc focus of the existing classifications. That is, the domain is structured through its individual elements without synthesis and coordination between them. If we cannot address this ad hoc issue, and if new studies continue to propose crowdsourcing taxonomies that are solely relevant to specific elements, the domain may end up with ambiguity over its structure. Given that, there is a strong need for a more comprehensive integrated approach in order to structure the domain.

Addressing the need, we suggest that a domain ontology and a process view are necessary for structuring the domain. Regarding the former, a domain ontology enables us to integrate the existing classifications. Nickerson et al. (2012) suggest that ontologies are the next stage of taxonomy development. Further, Corcho et al. (2003) highlight ontologies for their comprehensiveness and ability to structure domain knowledge. Regarding the need for a process view, we note that the existing classifications have not been linked together yet, which is necessary to constitute the whole crowdsourcing practice. This highlights the process view connecting individual elements in a meaningful way. This process view is a central of the dissertation, where we address crowdsourcing processes and BPC, and is the focus of the next section. 


\subsection{A Process Perspective on Crowdsourcing}

Two central concepts of the dissertation are the crowdsourcing process and BPC. This section is organised around them. The section starts with describing crowdsourcing processes in general, followed by an initial conceptualisation of business process crowdsourcing.

\subsubsection{Crowdsourcing Process}

The notion of a crowdsourcing process is critical to operationalise a crowdsourcing strategy. Thus, it is a recurrent topic in the crowdsourcing literature. We stress here the etymology of the word 'process', which refers to a systematic course of actions to accomplish some deliberate results. Well-coordinated processes are assumed not only to produce better crowdsourcing results, but also to deploy applications faster and with less cost (Tranquillini et al., 2015). The topic has attracted a lot of attention from researchers. By and large, research on crowdsourcing processes can be classified into two views: high and low levels of granularity.

With high granularity, a few studies adopt a holistic view to conceptualise the crowdsourcing process. Early, research referred to crowdsourcing processes with an understanding purpose. Consequently, crowdsourcing processes were conceptualised by rich descriptions with several illustrative examples (Leimeister, Huber, Bretschneider, \& Krcmar, 2009; Whitla, 2009), and by identification and description of actions executed by different crowdsourcing actors (Geiger et al., 2011; Vukovic, 2009; Wexler, 2011). At this early time, crowdsourcing processes were mostly studied together with other foci like crowdsourcing applications and taxonomies, rather than as a separate primary research focus. Before moving to review studies that primarily investigate crowdsourcing processes, we synthesise the existing descriptions to provide a narrative sketch of the crowdsourcing process. More precisely, we adapt the earliest but most widely used description by Whitla (2009) and add into it supplementary descriptions. As a result, a crowdsourcing process can be described as follows.

When an organisation has tasks to be accomplished, the first step is to decide whether to choose crowdsourcing to perform these tasks or not (Wexler, 2011). If the decision to crowdsource is made, the organisation then defines the tasks and creates an open call to 
release the tasks to the crowd. This step can be done through a platform developed either by the organisation or by a third party. Through the platform, the organisation can approach members of the crowd. Depending on the organisation's requirements, the members can be specific to a particular community or just anyone willing to perform the task. Accomplishing these tasks individually or collaboratively, the members then submit the results back to the organisation, which assesses the quality of the results. Payment or other incentives will be given to the members if the organisation is satisfied with the results (Whitla, 2009). The results are intended to be incorporated into organisational activities (Leimeister et al., 2009; Wexler, 2011).

Keeping in mind the initial descriptions, researchers started to explore crowdsourcing processes from a high level of granularity. Aiming to identify the main structures of the process, they commonly adopted an abstract view to discover the main stages and concerns in the process. Brabham $(2009,2012 \mathrm{~b})$, exploring a crowdsourcing project for public participation in transit planning, formulates a crowdsourcing process using four stages. First, a problem that needs to be solved and its related information are clarified. Second, an open call is sent to the crowd through a self-developed website. This call includes data necessary to solve the problem, reward information and the intended format of the solutions. Third, crowd members can choose to participate in the project. Finally, the organisation evaluates the proposed solutions to choose the winners.

Also adopting an abstract broad view, Muhdi et al. (2011) conducted an explorative case study to analyse twelve crowdsourcing projects. As a result, they formulate the main operations in the crowdsourcing process as five stages: deliberation, preparation, execution, assessment, and post-processing. In the first stage, organisations analyse crowdsourcing and "decide whether the crowdsourcing approach is appropriate to solve their internal problem[s]" (p. 322). If the decision to crowdsource is made, the second stage involves choosing a particular platform that is appropriate for the crowdsourcing activity. The next two stages are dedicated to executing the crowdsourcing activity on the chosen platform, and evaluating the received results. The final stage transfers the received results, such as ideas and solutions, to real organisational implementation.

In a similar vein, Stol and Fitzgerald (2014) conducting case study research recently examined crowdsourcing processes in the context of software companies. However, they 
structure their findings differently compared to the two aforementioned studies. More precisely, instead of formulating crowdsourcing processes as a set of sequential stages, they identify major building blocks of the crowdsourcing process, including task decomposition, coordination and communication, planning and scheduling, quality assurance, managing knowledge and intellectual property, and providing incentives to the crowd. Similar approaches that formulate main elements of the crowdsourcing process by case study are quite common (Ågerfalk et al., 2015; Zogaj et al., 2014).

Overall, this group of studies views the crowdsourcing process as an important research focus and contributes empirical efforts to formulate the main stages and building blocks that comprise the crowdsourcing process. However, the main research methods adopted in these studies are exploratory case studies (Ågerfalk et al., 2015; Muhdi et al., 2011; Stol \& Fitzgerald, 2014; Sutherlin, 2013). The exploratory nature, together with the particular investigated cases/contexts, explains the existence of different, likely one-off crowdsourcing processes. Furthermore, as this group of studies aims at painting an overall picture of the crowdsourcing process, they tend to focus more on high-level abstract concepts and thus face significant gaps mapping the abstract concepts to specific workflows or activities, necessary to establish the crowdsourcing process.

With low granularity, a large number of studies have investigated varied aspects of the crowdsourcing process. Although they have helped specify workflows and activities necessary to establish the crowdsourcing process, their ad hoc nature has been repeatedly highlighted in the domain (Geiger \& Schader, 2014; Man-Ching et al., 2011; Zhao \& Zhu, 2014). This ad hoc nature is further revealed through two aspects. First, different research methods have been adopted to examine the specific activities of the crowdsourcing process. For instance, methods for researching task definition include lab experiments (Khazankin et al., 2012a), open-ended and quantitative surveys (Schulze, Seedorf, Geiger, Kaufmann, \& Schader, 2011), and engineering design (Bozzon, Brambilla, Ceri, \& Mauri, 2013). These differences contribute to clarifying different aspects of the activity, yet a comprehensive approach is still missing. Second, the domain is lacking a strong knowledge base guiding crowdsourcing process establishment (Palacios et al., 2016; Zhao \& Zhu, 2014). As a result, the domain knowledge remains scattered, varied and sometimes conflicting. 
Given the existence of the large number of studies in this group, this section does not intend to review them one by one, which will be the focus of the scoping knowledge source in Section 4.1. Rather, we summarise other major literature reviews, which characterise the complexity and isolated concerns of the crowdsourcing field. Although a few literature reviews have been conducted in the domain, we focus on the two most recent and major reviews.

In 2014, the first major review was published by Zhao and Zhu (2014). These authors identified 55 crowdsourcing papers, based on a systematic search and selection of all major scholar databases in the period from 2006 to 2011. Analysing the papers, they suggest that "empirical studies have been conducted almost entirely on events/processes" (p. 419). These authors further map these ad hoc foci into major themes, and outline future research directions, including motivation to participate, participant's behaviour, making the decision to adopt crowdsourcing, governance and implementation, quality control and evaluation, incentive mechanisms, and technological issues, which are all major topics of crowdsourcing process studies. The review also indicates the emerging nature of the domain because only a small part of the studies $(16 \%)$ is based on theoretical foundations. Amrollahi (2015), among the most recent reviewers, aims at synthesising the crowdsourcing literature into a process model. He started by searching crowdsourcing papers in the period of 2009 to early 2014, coming up with 566 papers, and then selected 39 papers directly focusing on the crowdsourcing process. The review contributes to a better understanding of the crowdsourcing process in three ways. First, it proposes a process model to structure the crowdsourcing process. To an extent, the model is more or less aligned with the stages of the crowdsourcing process described in the aforementioned review. Second, the review indicates a strong development of the field, with a significant increase in the number of papers published recently (566 papers). Lastly, Amrollahi (2015), aligning with Zhao and Zhu, concludes the ad hoc feature of the current literature, and further highlights that crowdsourcing process research remains scarce, with only 39 related papers that can be identified out of the 566 papers found. 
In summary, research has studied the crowdsourcing processes from both high and low levels of granularity. With high granularity, a few studies adopt an abstract conceptualisation when exploring a crowdsourcing process. As a result, these studies identify main stages and issues that should be considered in the crowdsourcing process. They contribute to the structures of the crowdsourcing process, which enable us to incorporate an analytical framework discussed in the next major section of this review. However, it is important to note that these studies are more focused on highly abstract conceptual understanding and thus detailed activities are still missing.

With low granularity, a larger number of studies examine individual processes/events from varied deconstructed aspects. They provide various contributions, reported in case studies, expert opinions, usability studies, experiences, and other engineering development. Though realising the importance of the high-level view, they tend to investigate only parts of the process. The ad hoc nature of these studies are commonly complained and are confirmed by the two major reviews in the domain. Furthermore, these reviews highlight that research into the crowdsourcing process as a whole is scant, something also suggested by others (Hossain et al., 2015; Pedersen et al., 2013). As a result, the domain is still unstructured and lacks "a comprehensive guideline through which practitioners can initiate and manage their crowdsourcing projects" (Amrollahi, 2015, p. 2).

To conclude, a few studies cover the crowdsourcing process as a whole without its parts, while a large number of studies investigate the concept through its parts without the whole. The domain is characterised by a large number of ad hoc knowledge sources, which are scattered, varied and sometimes conflicting. This indicates the lack of a solid knowledge base founding the crowdsourcing process. What is also missing is an integrated view of the two levels of granularity, which can provide a complete picture on decomposed activities of the crowdsourcing process and their coordination.

Such an integrated view can be achieved through a business process lens, which has rarely been adopted in the crowdsourcing field. This points us to the concept of business process crowdsourcing, conceptualised in the following section. 


\subsubsection{Business Process Crowdsourcing}

This section explores business process crowdsourcing (BPC). As this concept is relatively new, the review is limited to a small amount of existing relevant literature.

Based on the need for an integrated picture of crowdsourcing processes, this dissertation investigates crowdsourcing using a business process lens. We designate this particular view as Business Process Crowdsourcing (BPC). The term was first coined by La Vecchia and Cisternino (2010) to refer to a model allowing organisations to send their internal business processes to the crowd, as an alternative to business process outsourcing (BPO). We further define the concept as a way to establish repeatable organisational business processes based on crowdsourcing. Etymologically, BPC combines the phrase business process with the word crowdsourcing. We elevate the business process construct to be equally important as the crowdsourcing construct. According to van der Aalst and Hee (2004), a business process is defined as a collection of individual activities and a workflow coordinating them. It aims to achieve a particular goal with both effectiveness and efficiency. A business process is purely conceptual, yet it serves as a template for creating multiple, real life instances of the same process, which organisations may create repeatedly and concurrently.

Given that, this dissertation defines BPC as a set of activities completed by crowdsourcing entities, in conjunction with a coordination of these activities, that collectively form the entire business process.

Our proposition is that BPC proposes an efficient integrated approach for organisations to establish a crowdsourcing process. This efficiency is realised through three roles. First, BPC can help streamline internal and external tasks in the crowdsourcing strategy. More precisely, the BPC perspective enables analysis of both individual aspects of crowdsourcing and coordinating them into an organisational workflow (La Vecchia \& Cisternino, 2010; Lüttgens et al., 2014). By establishing BPC with well-organised workflows, organisations can integrate the crowdsourcing strategy with their day-to-day business processes (Tranquillini et al., 2015). Thus, it enables the incorporation of the crowdsourcing capabilities into the organisational value proposition.

Second, BPC also enables us to standardise crowdsourcing processes. Process standardisation can only be achieved by comprehending all related activities and their 
relationships. BPC that relies on both the individual and coordinated views is in a unique position for this comprehension. More precisely, BPC can provide both a detailed view to understand the deconstructed aspects, and a holistic view to understand their relationships, both necessary for process standardisation. This is similar to the role of the business process view on standardisation of outsourcing (Wüllenweber, Beimborn, Weitzel, \& König, 2008).

Finally, BPC contributes to move crowdsourcing to a more well-defined status. The current ad hoc status of the domain has been noted and discussed in the previous section of the review. Bringing a business process lens to crowdsourcing, BPC allows analysing and defining the basic workflows of crowdsourcing processes, and enabling us to build crowdsourcing processes on top of existing business process management (BPM) technology (Khazankin et al., 2012a; Satzger, Psaier, Schall, \& Dustdar, 2011; Tranquillini et al., 2015). In this sense, BPC is expected to efficiently establish crowdsourcing as a common organisational practice, and move crowdsourcing processes to a more well-defined status.

Given these important roles, there are many calls for further studying BPC, especially how to conceptualise and establish it. Vukovic et al. (2010) are among the earliest researchers that ask "how does crowdsourcing become an extension of the existing business process" (p. 7). Khazankin et al. (2012a) echo this question and complain about "the lack of an integrated way to execute business processes based on a crowdsourcing [platform]" (p. 1). Similarly, Lüttgens et al. (2014) have recently emphasised the need to build a dedicated process for crowdsourcing. The need for BPC can also be implied from the fact that organisations have utilised crowdsourcing for some of their core, complex processes, such as product development (Djelassi \& Decoopman, 2013) and industrial processes (Muntés-Mulero et al., 2013), which should be necessarily linked with other internal business processes.

In spite of these calls, there has been little investigation into BPC and thus how to establish BPC has not been fully examined in the literature. Some prior studies have touched different aspects of BPC. Satzger et al. (2011) seek to help organisations "fully automate[d] deployment of their tasks to a crowd, just as in common business process models" (p. 67), but focus only on choosing suitable workers to perform tasks. Similarly, 
Khazankin et al. (2012a) highlight the need for organising business processes based on crowdsourcing, but they investigate only a part of the problem, which is how to optimise task properties for supporting business process execution.

A few recent models/frameworks conceptualising crowdsourcing processes contribute to the understanding of BPC. One of the earliest model is proposed by Pedersen et al. (2013). From a process perspective, they in-depth analysed existing research in the domain for conceptualising crowdsourcing. As a result, they propose a conceptual model, organised as an Input-Process-Output structure. The model explains key dimensions of crowdsourcing, including problems, technology, processes, governance, people, and outcomes, which provides a starting point for further study on crowdsourcing processes.

Also analysing existing research in the domain, Hetmank $(2013,2014)$ aimed at understanding crowdsourcing systems and their components. For this purpose, he suggests a model comprising of four components: user management, task management, contribution management and workflow management (Hetmank, 2013). Based on the identified components, Hetmank (2014) further proposes a lightweight ontology defining vocabularies of crowdsourcing systems. The vocabularies specify classes and properties, which are useful for crowdsourcing system development. Yet, further evaluation is needed to empirically test the proposition before its practical use, as noted by the author (Hetmank, 2014).

The crowdsourcing process has also been modelled using BPM technology. Tranquillini et al. (2015), based on Business Process Model and Notation (BPMN) technology, modelled workflow patterns of the crowdsourcing processes. They also designed a runtime environment operating these patterns in order to support the workflow enactment. As a result, the study offers a modelling language supporting crowdsourcing workflow enactment and a visual editor that allows organisations to graphically create and manage their crowdsourcing processes. We note that although this work can enact, prototype, and configure a crowdsourcing process, it can only maximise its contribution with the assumption that organisations have already had clear structures of the crowdsourcing process. In other words, this work provides useful supports to configure business process crowdsourcing, which can only be possible if BPC can be clearly established. This further highlights the role of BPC establishment. 
Overall, since crowdsourcing needs to evolve from an ad hoc one-off process, we bring the business process lens to research crowdsourcing. We have introduced the concept of BPC and described its possible roles in moving crowdsourcing processes forward. Given these roles, many researchers have suggested further examination of BPC. However, there have been few attempts to do this, and even fewer attempts to establish and support BPC. These attempts have led to a few models/frameworks of crowdsourcing processes, but these models mainly focus on technical aspects of crowdsourcing systems rather than the business processes operating on these systems. Furthermore, most of the proposed models so far have not yet been empirically evaluated (Amrollahi, 2015; Hetmank, 2013). Thus, what is largely missing in the literature is an informed way to establish BPC, from conceptualising, to modelling, and to empirically supporting BPC establishment.

When initially conceptualising BPC, we note that an antecedent must exist to enable the BPC concept. That is, that exists repeatable building blocks of crowdsourcing processes, which collectively serve as a template for creating multiple, real life instances of the crowdsourcing process. From the preceding review, we have observed several processes, activities, and components that have been repeatedly discussed and thus can possibly be synthesised into the repeatable building blocks of BPC. The following section explores this possibility, leading us to identify the three highly abstract building blocks.

\subsection{An Analytical Framework of BPC}

According to Miles et al. (2014), research normally starts with a conceptual framework that broadly defines concepts of interest and their relationships that will be investigated. Investigating BPC, we now present an analytical framework decomposing the concept into its researchable foci. Such an analytical framework will, later on, be used to support our analytical process when we analyse a large number of knowledge sources in the domain to identify repeatable business processes of crowdsourcing. The framework mainly draws on the literature reviewed in the previous sections.

We start with an abstract view on crowdsourcing activities discussed in Section 2.3, which, by and large, can be grouped into three high-level stages: decision to crowdsource, design, and configuration. A crowdsourcing process logically starts with a managerial 
decision to crowdsource or not. This managerial decision considers the appropriateness of crowdsourcing to enhance existing organisational tasks (Afuah \& Tucci, 2012; Muhdi et al., 2011). After the decision to crowdsource, design concerns a set of decisions that have to be made to instantiate a concrete crowdsourcing process. We use the term design to highlight the fact that multiple instantiations are possible and that choice depends significantly on subjective criteria. Configuration concerns the materialisation of a design into a concrete system (Kittur, Smus, Khamkar, \& Kraut, 2011; Little, Chilton, Goldman, \& Miller, 2010). These three stages are well aligned with the traditional information systems development lifecycle, usually divided into plan, design and development phases (Saldarini, 1989). They constitute the analytical framework that presents a high-level, logical view of the crowdsourcing process. It is graphically presented in Figure 2.3.
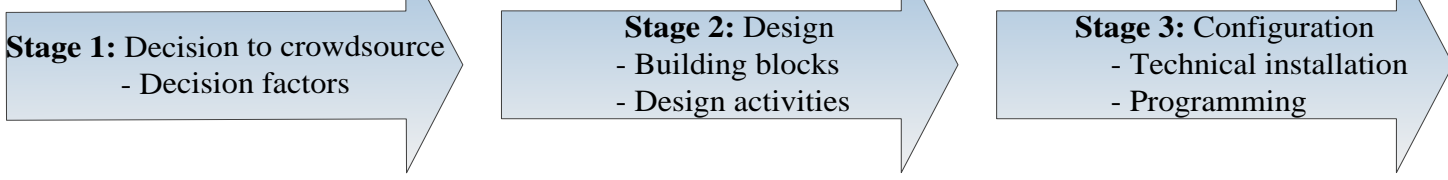

\section{Figure 2.3. Three-stage analytical framework}

Each stage of the framework and their main concerns are discussed below.

\subsubsection{Decision to Crowdsource}

The reviewed literature suggests that a crowdsourcing activity begins with the decision to crowdsource, which considers whether crowdsourcing is an appropriate approach to accomplish the organisational tasks. Indeed, Muhdi et al. (2011) and Wexler (2011) position this decision in the first stage of the crowdsourcing process. A similar position and purpose of the crowdsourcing decision is explicitly stated by other researchers (Djelassi \& Decoopman, 2013; Lüttgens et al., 2014).

The decision to crowdsource plays a central role in a crowdsourcing strategy, for several reasons. First, this decision links directly to whether an organisation will open or close their business processes to the crowd (Seltzer \& Mahmoudi, 2013). Second, it affects the use of organisational resources, at least the resources dedicated to crowdsourcing, because inappropriate decisions are likely to lead to unplanned challenges (Rouse, 2010). Furthermore, as a special kind of project that links to the crowd, a failed crowdsourcing project caused by the decision to crowdsource will have a negative impact on the organisation's reputation. Finally, with its starting position, the decision to crowdsource 
cannot be changed in the latter stages of the crowdsourcing process, and thus it greatly influences the success of the entire crowdsourcing activity (Muhdi et al., 2011).

With this crucial role in mind, researchers have already begun to investigate the decision to crowdsource, focusing on factors driving this decision. Some studies, maybe for simplification, examine one single factor to suggest when organisations should crowdsource. For instance, Ranade and Varshney (2012) addressed the question "to crowdsource or not to crowdsource" (p. 1) by mainly focusing on the nature of tasks. Naroditskiy et al. (2013) considered "the trade-off between the potential for increased productivity with the possibility of being set back by malicious behaviour" (p. 1). However, more recent studies recommend examining potential benefits and risks of crowdsourcing (Lu, Hirschheim, \& Schwarz, 2015; Muhdi et al., 2011) and weighing organisational contexts against general crowdsourcing capabilities (Djelassi \& Decoopman, 2013). Consequently, multiple factors should be considered when making a crowdsourcing decision (Afuah \& Tucci, 2012; Lu et al., 2015; Thuan, Antunes, \& Johnstone, 2016; Zhao \& Zhu, 2014).

\subsubsection{Design}

After organisations decide to crowdsource, they need to transfer this decision to concrete designs. Design is defined, according to Hevner and Chatterjee (2010), as a plan for structuring elements in order to best accomplish a particular purpose. Adopting this definition, the design stage should plan and structure activities of a crowdsourcing process. It is here the BPC view should maximise its benefits. In other words, this stage should identify both the abstract building blocks, and the detailed design activities and related information structures of the crowdsourcing process.

The literature has shown several possible building blocks of BPC and their detailed design activities. For example, Section 2.3 has reported a few building blocks proposed by Pedersen et al. (2013) and Hetmank (2013). Another example is the list of design building blocks and activities suggested by Kittur et al. (2013), who research crowdsourcing on complex, large-scale tasks. This list includes twelve abstract activities, including workflow design, task assignment, hierarchy, real-time response, collaboration, quality control, crowds guiding artificial intelligence, artificial intelligence guiding crowds, platforms, task design, reputation, and motivation. Other design building blocks and their 
detailed activities can also be found in the literature (Ågerfalk et al., 2015; Amrollahi, 2015; Stol \& Fitzgerald, 2014; Zogaj et al., 2014).

Given the existence of different building blocks and their detailed design activities, we note here three important points. First, the differences, again, confirms the ad hoc nature of the domain, and thus suggest a more comprehensive integrated approach to synthesise these building blocks. Second, these studies have highlighted the need to design the different building blocks and activities of the crowdsourcing process. That is, to establish a crowdsourcing process, several activities of the crowdsourcing process need to be designed and structured, which suggests the role of process design (Stage 2 of the framework). Finally, despite the differences of the proposed building blocks and activities, we can identify some repeatable activities, such as crowd management (Kittur et al., 2013; Pedersen et al., 2013) and quality control (Amrollahi, 2015; Kittur et al., 2013). Consequently, it is possible and necessary to reconcile the differences and suggest common building blocks of how to design the crowdsourcing process.

\subsubsection{Configuration}

The configuration stage transforms a crowdsourcing design into a concrete implemented system. In the crowdsourcing context, configuration can refer to either technical decisions to set up crowdsourcing components on existing platforms (Gonnokami, Morishima, \& Kitagawa, 2013; Hosseini, Phalp, Taylor, \& Ali, 2015a; Kittur et al., 2011; Little et al., 2010), or in-depth technical software development to build a crowdsourcing platform, such as algorithms, protocols, and database structures (Schall, 2012). Although this stage can be considered from both views, the chosen business process perspective limits our concern within the process configuration on an existing platform. This is also supported by the availability of several crowdsourcing platforms (Hirth et al., 2011) and programming toolkits that eases the configuration (Kittur et al., 2011; Kucherbaev et al., 2013; Little et al., 2010; Tranquillini et al., 2015).

Overall, we have structured the three-stage analytical framework initially conceptualising BPC. The framework structures three high-level stages of BPC: decision to crowdsource, design, and configuration, which will be deconstructed into the main building blocks and activities to thoroughly conceive the concept. The framework will guide our data collection and data analysis by providing a focus for these research activities. 


\subsection{Chapter Summary}

This chapter has provided a narrative review to assess the state of the art that driving the dissertation to study BPC. One main drive is that the crowdsourcing domain is emerging, characterised by unstructured knowledge sources and the lack of a strong knowledge base. There appears to be evidence for this in the literature reviewed in the previous sections.

The review covered four major strands. The first strand examined the conceptualisation of crowdsourcing. It shows three main pillars behind the concept, followed by a discussion in order to compare and contrast crowdsourcing with other related concepts. They draw a boundary around the crowdsourcing concept and show that crowdsourcing is a distinctive concept per se. Then the short history of crowdsourcing definitions was discussed to show that the concept continues to evolve. Together, while the distinctive concept suggests that crowdsourcing needs to be independently developed, the evolution of the concept's definitions and its short history indicate the emerging nature of crowdsourcing.

The second strand reviewed basic classifications in the crowdsourcing domain. This shows that research into classifications cover many particular topics: applications, tasks, types of crowd members and platforms, but not yet cover the synthesis and coordination among them. It is the ad hoc nature of the domain, where classifications are solely relevant to particular crowdsourcing elements or contexts. Further, these classifications have not yet been synthesised and linked in a comprehensive integrated structure, and thus there is still a need for a solid knowledge base that structures the domain.

Third, the review covered the literature on crowdsourcing processes and business process crowdsourcing. It started with the two major research streams of crowdsourcing processes: high and low levels of granularity. It finds that some studies research crowdsourcing processes as a whole without its parts, while a large number of studies investigate specific parts of crowdsourcing processes without the whole. The different levels of granularity have hindered us to have a completed picture of crowdsourcing processes. Further, major reviews of crowdsourcing literature show that the domain is characterised by a large number of scattered, varied and sometimes conflicting knowledge 
sources. Addressing this challenge requires an integrated view, which has led us to introducing BPC.

Reviewing what little has been published on BPC highlights three important points. First, the review introduces the concept of BPC that brings a business process lens to study crowdsourcing, which enables us to establish crowdsourcing as an organisational business process. Second, the review discusses the roles of BPC. It shows that BPC can resolve the ad hoc challenge and provide structures for the domain. Finally, it finds a few models and frameworks contributing to understand crowdsourcing processes, but not comprehending BPC. Together, these points suggest that BPC is an emerging yet important phenomenon that needs to be conceptualised, modelled, and applied to crowdsourcing practices.

The final section of this chapter developed an analytical framework presenting the three abstract stages of BPC: decision to crowdsource, design, and configuration. The framework abstractly conceptualises BPC, and will be used to guide our data collection and analysis for further conceptualising and structuring BPC.

The following chapter discusses design science and the research method for this study. 


\section{RESEARCH DESIGN}

A research design provides an essential way to address the research questions. The design needs to be explicit in its research paradigm and research method that includes a systematic process of research activities (Van Aken, 2004). This study adopts the design science paradigm (Hevner \& Chatterjee, 2010; Hevner et al., 2004). In this paradigm, a sound design process will be applied to rigorously develop and verify the artefacts, addressing the research questions.

This chapter justifies and explains the research design, structured as follows. First, the design science paradigm is discussed. Second, existing design methods are reviewed, focusing on why these methods may or may not be appropriate to address the research problem. Third, a research process, designed to rigorously address the research questions, is described. Finally, a summary of the overall chapter is presented.

\subsection{Design Science Paradigm}

A research paradigm is a set of beliefs about how a problem of interest should be understood and studied. It is a combination of research questions being asked, research methods addressing these questions, and nature of pursued research outcomes (Van Aken, 2004). Understanding this combination is useful because it influences the choices in the research design.

The IS discipline has been evolving towards two basic research paradigms: behavioural science and design science (Hevner \& Chatterjee, 2010; Hevner et al., 2004; March \& Smith, 1995). Behavioural science seeks to find the truth in order to describe and predict an IS phenomenon around the analysis, design, implementation, and use of information systems. This paradigm, having a close relationship to natural science, is centred on understanding the phenomenon (Niehaves, 2007). Further, it is also characterised by its orientation to develop or test theories handling the understanding (Winter, 2008). Thus, main research outcomes in this paradigm are theory building and theory testing. 
Design science has been embraced as an acceptable research paradigm, being more focussed on utility than truth, and often regarded as complementary to behavioural science (Hevner \& Chatterjee, 2010; Hevner et al., 2004). Besides the emphasis on utility, design science research is also characterised by addressing the conceptualisation and development of innovative artefacts, which are intended to solve wicked problems (PriesHeje \& Baskerville, 2008). In other words, design science is fundamentally a problemsolving paradigm. Another fundamental pillar of design science emphasises a rigorous approach to advance current knowledge on innovation, design and development (Gregor \& Hevner, 2013; Hevner et al., 2004). All in all, this combination of utility, innovation and problem solving places design science as a distinctive research paradigm.

In this research, we adopt the design science paradigm, which is driven by three factors: the nature of the research questions, the nature of the phenomenon of interest, and the researcher's perspective (Orlikowski \& Baroudi, 1991). Design science is appropriate to address the research questions in this study. Specifically, our research questions focus on 'construct' and 'support', which are distinctive emphases of design science providing prescriptive knowledge to say how to construct, support, and do something (Gregor, 2006; Gregor \& Hevner, 2013). In addition, the final ultimate research question leads to construct a decision tool supporting the establishment of BPC. The high percentage of decision support systems (DSSs) that have adopted the design science paradigm (Arnott \& Pervan, 2014) further suggests design science is a suitable paradigm for addressing the research questions.

Second, the phenomenon of interest, BPC, also leads us to the choice of design science. At the first glance, this phenomenon could have been studied from a behavioural science paradigm as well. While behavioural science promotes several exploratory research methods to explore emerging phenomena like BPC, it is not rare that design science can also be adopted. This is because the emerging nature of the phenomenon indicates an illdefined research phenomenon, which is highly aligned with the advantage of design science addressing wicked problems (Pries-Heje \& Baskerville, 2008). Further, the practicality of the BPC phenomenon also suggests the use of design science. More precisely, crowdsourcing, and in particular BPC, spread from practices to academics (Howe, 2006a; La Vecchia \& Cisternino, 2010). This practical nature emphasises the 
choice of design science, strongly relevant to address practical challenges (Hevner \& Chatterjee, 2010).

As a matter of fact, the researcher has a set of beliefs closely aligning with the design science paradigm. The researcher views design science as an interesting research paradigm that may largely contribute innovation and novelty to the IS discipline. In this line, the researcher supports Hevner and Chatterjee's (2010) assertion that the adoption of design science contributes to clarifying the boundary of the IS discipline, distinctive from social science and computer science. Thus, by using the design science paradigm to research BPC, we may add our (very) small contribution to positioning IS as a distinctive research discipline.

Based on the above justifications, this dissertation adopts the design science paradigm and uses the following definition provided by Hevner and Chatterjee (2010, p. 5).

"Design science research is a research paradigm in which a designer answers questions relevant to human problems via the creation of innovative artefacts, thereby contributing new knowledge to the body of scientific evidence".

Having adopted the design science paradigm, we now specify its main characteristics. First, design science research concerns both design products and design processes (Hevner et al., 2004). In the design product view, design science research must produce feasible artefacts. March and Smith (1995) suggest four main types of artefacts: constructs, models, methods, and instantiations.. Constructs provide the vocabularies for the domain of interest. Models use constructs to abstractly express their relationships. Methods are a set of defined steps that can be used to perform a task. Instantiations are realisations of constructs, models, and methods implemented in working or prototyping systems. In the design process view, research is defined through its activities, where the research systematically moves from the problem to the solution. March and Smith (1995) identify two main design science activities: build and evaluate. Build activities refer to the construction of artefacts that address the given problem. Evaluate activities, or simply evaluations, refer to the assessment of the output artefacts with respect to its utility in solving the problem.

Design science research is also characterised by managing relevance and rigour on top of design processes and design artefacts. More precisely, design science research is 
conducted to purposely address relevant business problems, and as a result, its outcome artefacts should be applied back to the business environment. Furthermore, the design process has to be rigorous in both the build and evaluate activities. It means that the research process needs to draw on the existing sound knowledge and methods in order to rigorously advance the design and to scientifically evaluate its artefacts.

Overall, design science research is characterised by building and evaluating innovative artefacts in a rigorous way to address the relevant problem. Although some extensions can be found, e.g. types of artefacts may include building theories (Venable, 2006), these characteristics have been echoed repeatedly in the literature (Gregor \& Hevner, 2013; Hevner, 2007; Niederman \& March, 2012; Peffers, Tuunanen, Rothenberger, \& Chatterjee, 2007; Winter, 2008). Thus, they are used here to guide the dissertation, having selected the design science paradigm.

\subsection{Design Methods and a Need of a Design Method for Emerging Domains}

As with other non-incumbent scientific paradigms, studies in design science should provide very methodical and transparent accounts of researchers' activities: conceptualising a design problem, analysing multiple solutions, making choices, building an innovative artefact, and evaluating its utility. This stresses the importance of design methods guiding the research activities. We now consider the existing design methods and assess why they are appropriate or inappropriate to the current study.

Several design methods have been proposed in the design science paradigm, which can generally be categorised into two main groups: abstract and contingency. The abstract group, which includes the works by Peffers et al. (2007), Iivari (2007), and Hevner et al. (2010; 2004), provides general guidance for design science research. For instance, Hevner et al. (2004) propose seven principles that should be adopted in design science studies. These principles were recently embedded into three cycles that every design science study should exhibit: relevance, design, rigour cycles (Hevner \& Chatterjee, 2010). The relevance cycle links the design activities to the application domain and therefore helps define the opportunities and requirements for the artefacts. The design cycle iterates 
between the build and evaluate activities. The rigour cycle grounds the design process on a rigorous knowledge base, which can be drawn from different sources, ranging from well-established theories, to existing artefacts, and to experience and expertise. Focusing on the design cycle, Peffers et al. (2007) propose a generic research process consisting of six stages: problem definition, identification of solution objectives, artefact design, demonstration, evaluation, and communication. The contributions from this group of generic methods are expected to influence a broad range of design science studies.

Nevertheless, the research community keeps exploring and developing new methods. A reason to advance new methods is the increasing variety and wickedness of design problems, which challenge researchers to find new ways to tackle them. As stated by Gregor and Hevner (2013), "there are likely to be different design practices depending on the type of artefact" (p. 350). Because of this variety and the complex nature of design, it seems unreasonable to look for a comprehensive 'one-size-fits-all' method.

Consequently, the second group adopts a contingency approach, targeting specific classes of problems and contexts with specific methods. Carlsson et al. (2011) propose a design method specifically targeting IS development from a governance perspective. The governance perspective is very different from, say, enterprise resource planning or business process management. Therefore, it seems right to adapt a specific method, which in the case includes four steps: define problem situations and outcomes, review extant theories, propose a design theory, and test the proposed theory.

Also in this group of contingent methods, Pries-Heje and Baskerville (2008) propose the Design Theory Nexus (DTN) as adequate to design contexts where several competing theories exist and where it is often difficult to rationally make a choice between them. Following a design-space analytical approach, the DTN considers alternative solutions derived from theory, analyses conditions under which these solutions optimise utility, formulates the conditions into assertions, and advances an IS artefact that incorporates all these analysed elements into a systematic decision-making process. Although these two methods have already been used in several studies, we note that they are more suitable for well-established areas, because they assume that the design problem can be scaffolded from pre-existing theory. In other words, design should be "based soundly on extant theories" (Carlsson et al., 2011, p. 114). 
Applying the existing design methods to study BPC in the current research seems inadequate. To clarify this argument, we recall the two main characteristics of the BPC domain identified in the literature overview chapter. First, the existing knowledge in the BPC domain is scattered. The review could not identify prevailing theories or solid knowledge bases; something that was also noted by other authors (Palacios et al.; Zhao $\&$ Zhu, 2014). Rather, there exists a relatively large number of multiple case studies, expert opinions, design experience, and prototyping developments. Second, the domain is characterised by diverse, ad hoc, and sometimes conflicting findings and implications (Geiger \& Schader, 2014; Hosseini et al., 2015a; Man-Ching et al., 2011). These characteristics have limited the application of the existing theory-centred methods to research emerging fields like BPC. Thus, we align with Miah et al. (2014) saying that in emerging fields like BPC "traditional design science development methods have several limitations in supporting businesses, including conceptual mismatches, static models and inflexibility" (p. 273).

Given this problematic situation, there is a need for design methods studying BPC and other emerging fields, characterised by scattered knowledge sources rather than accepted theories. This need is aligned with Hevner and Chatterjee (2010), who note that "to insist that all design decisions and design processes be based on grounded behavioural or mathematical theories may not be appropriate or even feasible for a truly cutting-edge design artefact" (p. 18). These authors also outline directions to address the problem. More specifically, they recommend that design science research can be rigorously founded on three types of knowledge sources: 1) scientific theories and methods; 2) metaartefacts; and 3) experience and expertise. Considering the BPC domain regarding the three types of knowledge sources, it is suggested that the predominant knowledge sources in the domain are expertise and experience, which exists in the form of individual sources of knowledge, including case studies, expert opinions, design and implementation experience, and prototyping developments. Consequently, we base this dissertation researching BPC on individual knowledge sources predominant in the domain. 


\subsection{Research Process}

This section defines a research process to guide the current research. As discussed earlier, this process grounds individual knowledge sources, which are highly available in the domain. When forming the research process, the researcher has gained many insights from the construction of the DTN, guiding how to connect alternative sources of theories (Pries-Heje \& Baskerville, 2008). In particular, the research process relaxes the requirement for using extant theories in the DTN, and instead considers existing knowledge sources in the BPC domain. Furthermore, we also need to address the inconsistent and conflicting findings in the BPC domain. This requires the research process to develop a domain ontology, which has been widely suggested to enable a shared consistent understanding in a domain (Corcho et al., 2003; Miah et al., 2014).

Given the discussion, together with the target to address the research questions, the research process follows four stages: scoping knowledge source, conceptual model, ontology, and decision tool. Figure 3.1 presents these stages and their aims.

The research process

\section{Scoping knowledge sources (RQ1)}

- Scoping literature review

\section{Conceptual model (RQ2)}

- Main building blocks

- Model construction

- Case study

\begin{tabular}{|c|}
\hline Ontology (RQ3) \\
- Ontology capture \\
- Knowledge organisation \\
- Triangulation \\
- Evaluation \\
\hline
\end{tabular}

\begin{tabular}{|l|}
\hline \multicolumn{1}{|c|}{ Decision tool (RQ4) } \\
- Prototype development \\
\hline \multicolumn{1}{|c|}{ Evaluation } \\
- Controlled experience \\
- Focus group
\end{tabular}

Figure 3.1. The research process

Aim

- Scope individual knowledge sources - Identify building blocks of BPC

- Structure the identified building blocks - Construct a process model of BPC

- Apply the model to two existing crowdsourcing projects

- Propose a domain ontology of BPC - Consolidate a knowledge base

- Compare the ontology with a version genereated by software

- Construct a decision tool supporting BPC establishment

- Quantitatively and qualitatively evaluate the tool usefulness 
The following sections justify the research stages in addressing the research questions. It also introduces and justifies the research techniques used in each stage, but keeps the details of these techniques in the relevant chapters to come. In the tenets of design science, the sections start with the build activities of each stage, followed by their evaluations.

\subsubsection{Build Activities}

The research process defines four build activities: scoping knowledge source, conceptual model, ontology, and decision tool. Following Hevner and Chatterjee (2010), all activities are based on rigorous research techniques. We now specify the nature of each activity, the techniques being used, and the activity role in addressing the research questions.

\section{Scoping Knowledge Sources}

The first research activity extracts and articulates the related knowledge sources in the domain. In the BPC domain, as individual sources of knowledge predominate over established theory, reviewing these sources helps create a foundation for enhancing knowledge in the current research (Paré et al., 2015; Van Aken, 2005). Another goal set by this activity is to analyse the knowledge sources for synthesising the main building blocks of BPC. The synthesis is based on what we name the 'wisdom of the researchers' where a collection of researchers is wiser than single experts, similar to the wisdom of the crowd (Surowiecki, 2004). As a result, this activity can identify the most salient building blocks of BPC in order to answer RQ1.

Regarding the specific technique of the activity, we note that various knowledge sources are reported in the crowdsourcing literature. Thus, we adopt a scoping literature review as the main technique of this activity. A scoping review that enables a comprehensive view on a particular topic is highly suitable for the emerging nature of BPC (Paré et al., 2015). More precisely, the 'scoping' classifier refers to a comprehensive sample strategy, which covers the vast range of knowledge sources existing in the domain. Another benefit of scoping review is its explicit process of how the review is conducted (Paré et al., 2015). This increases the level of transparency and rigour of the design science research. Having chosen a scoping review, we follow detailed guidelines on how to conduct a scoping literature review proposed by Okoli and Schabram (2010) and Paré et al. (2015). These studies explicitly explain the review steps, including how to search, filter, extract, and synthesise the related knowledge sources. 


\section{Conceptual Model}

The conceptual model grasps the main concepts and relationships in the domain of interest (Webster \& Watson, 2002). Consequently, the activity helps to identify and frame the structures of BPC building blocks, which addresses RQ2. Another expected result of this activity is a BPC conceptual model, where the main building blocks of BPC are structured in a meaningful way. In the design science paradigm, the conceptual model constitutes an IS artefact per se (Hevner et al., 2004).

To build the conceptual model, we apply Webster and Watson's (2002) procedure to draw the main concepts, processes, factors, and building blocks from the scoping knowledge sources for building and generalising a conceptual model. Among the extracted concepts, the conceptual model focuses on the dominant ones to keep the model both focused and representative. For this purpose, we adapt the following table from Webster and Watson (2002). In this table, each concept is specified through its name, definition, references, and total number of sources addressing the concept. The number can be used to identify the dominant concepts of BPC, e.g. building blocks, processes, and decision factors.

Table 3.1. $\quad$ Structure for summarising main concepts of BPC (adapted from Webster \& Watson, 2002)

\begin{tabular}{|c|l|l|l|}
\hline Concepts & Description/Definition & References & Total of supporting sources \\
\hline & & & \\
\hline
\end{tabular}

Based on Table 3.1, the researcher then analyses the knowledge sources for the main links between the main concepts. After identifying the concepts and relationships, the next step is to graphically represent them in a conceptual model, which is based on Jonker and Pennink's (2010) guidelines, including guides on how to sketch the concepts, draw relationships, and refine the model.

\section{Ontology}

The third distinctive activity is the ontology construction, which is critical to consolidate knowledge in the emergent domains like BPC. Several studies have suggested that ontologies can improve knowledge structures and understanding in the application domains (Guo, Schwartz, Burstein, \& Linger, 2009; Miah et al., 2014; Ostrowski, Helfert, \& Gama, 2014). Furthermore, building an ontology adds to the conceptual model in two important ways. First, it provides descriptive conceptualisation of the domain by 
explicitly articulating the concepts and their detailed sub-concepts, including building blocks, processes, activities, related information flows, and their relationships (Miah, Gammack, \& Kerr, 2007). As a result, the ontology can help resolve conflicts in the emerging domain by identifying whether several 'things' are represented by the same constructs (Shanks, Tansley, \& Weber, 2003). Second, the ontology provides an IS artefact for modelling the domain knowledge base, as suggested by Miah and von Hellens (2014), who used ontologies for representing the knowledge base of a DSS domain. All in all, the ontology can provide a solid BPC knowledge base and structure its elements, thus addressing RQ2 and RQ3.

By and large, ontologies can be classified into lightweight ones, which only capture concepts and relationships in the domain, and heavyweight ones, which add rules and axioms that constrain ontological elements (Corcho et al., 2003; Valaski, Malucelli, \& Reinehr, 2012). In our research process, we build a heavyweight ontology, which adds reasoning to the knowledge base. From a design science perspective, the reasoning knowledge allows comparing among alternatives for given issues (Pries-Heje \& Baskerville, 2008). From a DSS perspective (in relation to RQ4), the reasoning knowledge formulates decision parameters and prescriptive rules, and thus contributes to making informed decisions in BPC establishment (Miah et al., 2014).

The ontology literature has proposed several methods for building ontologies (Corcho et al., 2003; Pinto \& Martins, 2004). By reviewing these methods, we adopt two steps that are popularly used and aligned with our research process: ontology capture (Uschold \& King, 1995) and knowledge organisation (Küçük \& Arslan, 2014; López, Gómez-Pérez, $\&$ Corcho, 2004). The first step analyses data extracted from the scoped knowledge sources for ontological elements: concepts, hierarchical relationships, and decisionmaking relationships. This analysis is similar to the analysis in the conceptual model but at a more detailed level. As a result, Table 3.1 is transformed into a glossary table capturing the ontological elements, as suggested by López et al. (2004).

The knowledge organisation step then synthesises the ontological elements and structurally arranges them into the ontology. In immature application domains like BPC, conflicting findings on these elements may be found. To address these conflicts, the synthesis follows the 'wisdom of researchers' strategy, which states that the collective of 
researchers is smarter than the few. Thus, the ontological elements supported by many sources should be included in the ontology, while the others should be excluded. A similar strategy has already been applied to successfully develop ontologies in other areas (e.g. Osterwalder, 2004). These ontological elements then need to be organised in a manageable way. Some overall structures that can be considered for this activity are hierarchy structure, network structure, process structure, and layer structure. We note that this organisation is an iterative process where we may extend, clean up, and update the ontology several times.

\section{Decision Tool}

The final stage considers the decision tool development, which should support the decision makers in BPC establishment. At this stage, the tool should be built on the knowledge base provided by the ontology. The tool uses the ontological concepts for presenting its parameters, and the hierarchical relationships and decision-making relationships for guiding its reasoning. The tool may automate a set of constraints related to BPC establishment, and support the elaboration of different what-if scenarios. As a result, the to-be-built tool should satisfy the requirements of RQ4.

Given the dynamic nature of the emergent domain, we adopt a rapid prototyping method, which addresses the dynamism by iteratively developing and revising a few software prototypes (Kordon, 2002) and traversing the DSS design space (Lim et al., 2008). Furthermore, prototyping is appropriate for DSS development, as suggested by Miah et al. (2009) regarding the development of an expert system supporting rural business operators, and Antunes et al. (2014) regarding the development of a decision tool supporting geo-collaboration.

In summary, this section justifies the build activities of the research process: the scoping knowledge sources, conceptual model, ontology, and decision tool. It also specifies how to conduct these activities, which are based on the rigorous research techniques. The expected outcomes include scoped knowledge sources and a set of three built artefacts, which address the research questions. We also note that in the tenets of design science, these built artifacts need to be evaluated, which is explained in the next section. 


\subsubsection{Evaluate Activities}

Evaluate activities or evaluations are critically important in design science, equally important as the build activities. Although prior design science research often conduct evaluations only after an artefact is completely constructed, we align with the recent research and perform the build and evaluate activities concurrently (Hevner \& Chatterjee, 2010; Sonnenberg \& vom Brocke, 2012a; Winter \& Albani, 2013). This approach allows us to evaluate artefacts early, which ensures rigour and suggests early revisions if necessary. This approach is further important as the current study generates interrelated artefacts, and thus an evaluation and improvement of the prior artefact highly benefits the following artefact. Consequently, we view the research process as four stages looping of build and evaluate activities (left-hand side of Figure 3.1).

All artefacts generated from the build activities have to be evaluated. Besides the first stage, scoping knowledge sources, providing raw materials for the research, we evaluate the three artefacts: a conceptual model of BPC, ontology, and decision tool. Since different methods for artefact evaluation have been identified by researchers, including observation, expert evaluation, experiments, prototype, action research, case study, illustrative scenario, and focus group (Hevner et al., 2004; Peffers, Rothenberger, Tuunanen, \& Vaezi, 2012; Tremblay et al., 2010; Venable, Pries-Heje, \& Baskerville, 2016), here emerges a question of how to choose the appropriate ones to evaluate the artefacts in the current study. According to Venable et al. (2012), this choice should be based on several criteria, including the desired evaluation purpose, the nature of the artefacts, the desired level of rigour or precision, and practical constraints, including time, labour, facilities, and access to research objects. These criteria are used in the current research to choose and justify the adopted evaluation techniques: case study, triangulation, experiment, and focus group.

A case study approach, which empirically investigates a phenomenon within its realcontext (Yin, 2013a), is chosen to evaluate the conceptual model. This choice is driven by four reasons. First, the purpose of evaluation is to understand the use of the conceptual model in a practical context, i.e. crowdsourcing projects, and addressing contextual conditions is a strength of case study (Yin, 2013a). Second, case study enables in-depth and detailed examination of the model, which is necessary in the context of BPC with the immature knowledge base. Third, case study can provide empirical evidence, 
complementary to the conceptualisation of the model construction. Finally, the use of case study has been shown to effectively evaluate conceptual models, as used by other design science studies (Barclay \& Osei-Bryson, 2010; Currim \& Ram, 2012).

Triangulation is adopted to evaluate the ontology. Venable et al. (2012) suggest that design science evaluation helps: establishing artefacts' utility for achieving its stated purpose, comparing the artefact to other designed artefacts, and considering the sideeffects and weaknesses of the artefact for future improvements. Although the first approach could be used to evaluate the to-be-built ontology, it would require considerable efforts that may not be feasible in a $\mathrm{PhD}$ project. This research design chooses the second approach where it triangulates the different versions of the ontology. More precisely, the ontology is compared to another ontology generated by Ontogen (Fortuna, Grobelnik, \& Mladenic, 2007). Another reason for choosing this approach is a constraint of the dissertation size, its available time, and the requirement for a sole researcher to conduct the research, which minimises the advantages of the other approaches. Further, the triangulation evaluation increases precision through using software tools, complementary to the prior case study with high contextual realism.

To evaluate the decision tool, experiments and focus groups are used. As the decision tool is the final ultimate artefact of the research, the evaluation focuses on studying and quantifying its usefulness. Thus, a controlled experiment is an appropriate decision as it allows testing how much difference in performance there is (if any) between participants using the tool and others who do not use the tool. This difference indicates the usefulness of the tool. Regarding the nature of the artefact, the decision tool is an artificial instantiation, which Venable et al. (2012) suggest to be evaluated through experiments. Furthermore, the use of experiments to evaluate the decision tool is supported by Peffers et al. (2012), who find that the most common method for evaluating instantiated artefacts is experiments.

Besides the scientific evaluation of the decision tool through experiments, we also want to evaluate the usefulness of the tool as perceived by the end users. Thus, a focus group technique is also adopted to assess the tool regarding to participants' perceived usefulness. Focus group evaluation is suitable for providing qualitative data on the perceived usefulness (Tremblay, Hevner, \& Berndt, 2012). The qualitative data complement to the 
qualitative data collected from the experiments. Furthermore, this technique encourages group discussion and shared ideas regarding the utility of the artefact, which may be uncovered with other individual qualitative evaluation techniques like interviews. From a design science perspective, focus groups are rather new yet promising evaluation techniques (Tremblay et al., 2010). Based on these reasons, focus groups are appropriate for evaluating the decision tool.

Considering the four evaluation techniques in the research process, we note that they correspond different nature of evaluations. This is because the times when they occur in the research process are different (Antunes, Herskovic, Ochoa, \& Pino, 2012). More precisely, the case studies and triangulation, with an intermediate position in the research process, focus more on the internal construction of the artefacts in order to revise them and provide feedback for the subsequent construction. This intermediate position suggests a formative evaluation (Antunes et al., 2012). It means a constraint on the number of cases in the case studies, and limited versions of the ontology for the triangulation purpose. Conversely, the experiments and focus groups evaluate the decision tool as the ultimate outcome artefact. Thus, they take a role of summative evaluations (Antunes et al., 2012), where experiments are conducted with a larger number of participants and there is a combination of quantitative and qualitative data for evaluations.

In summary, four evaluate activities are used to assess the artefacts developed in the research process. We evaluate the conceptual model using case study, the ontology using triangulation, and the decision tool using experiments and focus groups. The rationale for using these techniques has been stated. Table 3.2 summarises the evaluation techniques and their justifications. The use of different evaluation techniques in design science research has been suggested by several researchers for ensuring the research rigour (Sonnenberg \& vom Brocke, 2012a; Venable et al., 2016). 
Table 3.2. Evaluation techniques

\begin{tabular}{|c|c|c|}
\hline Artefact & Evaluation Technique & Justification \\
\hline Conceptual model & $\begin{array}{l}\text { - Case study } \\
\text { (Formative) }\end{array}$ & $\begin{array}{l}\text { - Contextual understanding } \\
\text { - In-depth examination } \\
\text { - Empirical evidence } \\
\text { - Used by other design science studies }\end{array}$ \\
\hline Ontology & $\begin{array}{l}\text { - Triangulation } \\
\text { (Formative) }\end{array}$ & $\begin{array}{l}\text { - Precision } \\
\text { - Resource constraints } \\
\text { - Complement with the case study }\end{array}$ \\
\hline \multirow{2}{*}{ Decision tool } & $\begin{array}{l}\text { - Controlled experiment } \\
\text { (Summative) }\end{array}$ & $\begin{array}{l}\text { - Measuring the tool usefulness } \\
\text { - Quantitative data } \\
\text { - Most popular, particularly suitable } \\
\text { for evaluating instantiated artefacts }\end{array}$ \\
\hline & $\begin{array}{r}\text { - } \text { Focus group } \\
\text { (Summative) }\end{array}$ & $\begin{array}{l}\text { - Perceived the tool usefulness } \\
\text { - Qualitative data } \\
\text { - Discussion and idea sharing on the } \\
\text { artefact }\end{array}$ \\
\hline
\end{tabular}

\subsection{Ethical Considerations}

This research involves human participants in three research activities: case studies, experiments and focus groups. Before conducting each of them, a separate human ethics application was applied and approved by the Human Ethics Committee of the School of Information Management at Victoria University of Wellington. The approvals and relevant documents such as information sheets and consent forms are presented in Appendix B.

Following the principle of research ethics, informed consent and confidentiality were ensured in this research. All participants signed individual consent forms. In case the data were collected in Vietnam, the consent form and other research materials were translated into Vietnamese in order to inform the participants of the research nature. Regarding confidentiality, all collected data were kept confidential to the researcher, the research supervisors, and the translators who signed confidentiality agreements. The collected data 
were stored securely. Further, a summary of the research results was sent to the participants to check it disguised the participants and the organisations.

\subsection{Chapter Summary}

This chapter described the design science paradigm and justified the choice of the paradigm in the current study. The need of a design method for emerging research domains like BPC was specified. The research process was designed to address the research questions in the emerging BPC domain. This research process consisted of four stages: scoping knowledge sources, conceptual model, ontology, and decision tool. Each stage comprised both build and evaluate activities, except the first stage that did not produce an IS artefact and thus did not include an evaluation. The choice of the research techniques used in each activities were fully justified. Ethical approvals and considerations were described.

The details of the research activities and their results are presented in the next chapter. 


\section{THE FOUR RESEARCH STAGES OF BUILD AND EVALUATE}

Four research stages form the backbone of this research. The purpose of this chapter is to explain these four stages in detail, and to operationalise them into their build and evaluate activities. Consequently, the chapter is structured as four sections, around the four stages. Each section begins with an overview. Then it presents the build activity, outcome artefacts, and artefact evaluation. An exception is the first section of scoping knowledge source, which provides the knowledge base for the other stages and thus does not include an outcome artefact or evaluation activity. The chapter outline is summarised below.

+ Stage 1: Scoping Knowledge Sources

○ Scoping process

- Results of the scoping process

○ (No evaluation activity)

+ Stage 2: Conceptual Model

- A process model for BPC establishment

- Model evaluation using case studies

- Overview of the approach

- Case study design

- Case study results

+ Stage 3: Ontology

○ Building a BPC ontology

○ An ontology of BPC

- BPC ontology evaluation using triangulation

$+\quad$ Stage 4: Decision tool

- Architecture and decision tables

- Tool development

○ Tool evaluation using experiments

- Overview of the experimental approach

- Experiment design

- Experiment results

○ Tool evaluation using focus groups

- Overview of the focus group approach

- Focus group design

- Focus group results 


\subsection{Stage 1: Scoping Knowledge Sources}

Scoping knowledge sources was the first stage of the research process, which aimed to extract and scope knowledge sources in the BPC domain. As different knowledge sources like case studies, design solutions, engineering activities and expertise opinions have been reported in the BPC literature, a scoping literature review was adopted to build a firm foundation for enhancing knowledge of the domain. The scoping review method, which employs an explicit, comprehensive procedure for searching and analysing the literature (Levac, Colquhoun, \& O’Brien, 2010; Paré et al., 2015), helped to systematically identify and synthesise the different knowledge sources in the BPC domain. The synthesised knowledge provided the knowledge base for the research (Hevner \& Chatterjee, 2010), and thus contributed to addressing RQ1.

To begin the scoping review, this stage established a systematic process to ensure the rigour of the review results. We based the review process on the recommendations of how to conduct a good IS literature review, and especially, a good scoping review (Okoli, 2015; Paré et al., 2015). Following Okoli's (2015) recommendations, we adopted the five steps, including selecting sources, filtering sources, classifying sources, data extraction, and data synthesis. Figure 4.1 summarises the five steps of the scoping review, which are specified below. 


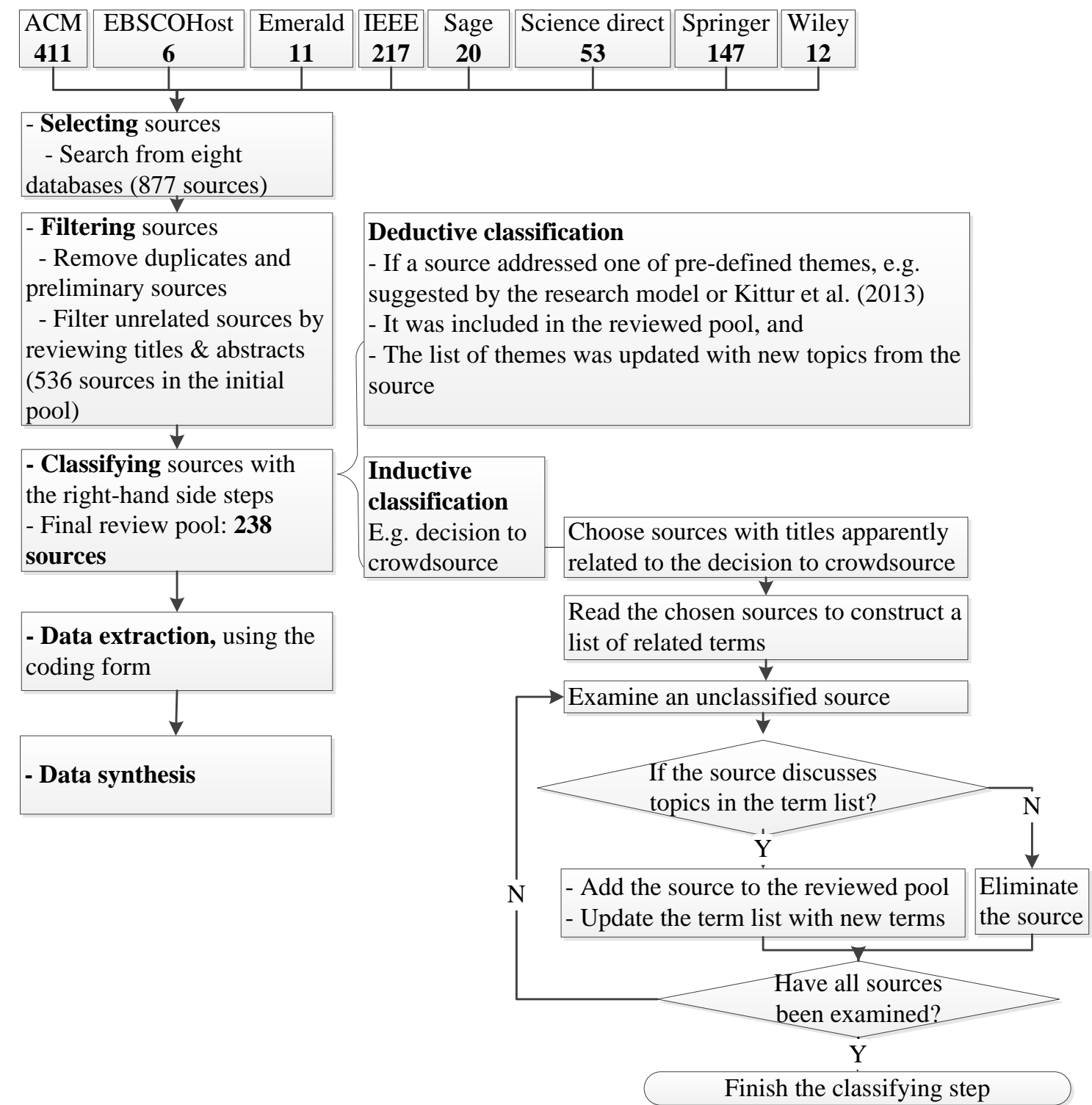

Figure 4.1. $\quad$ Steps and summary of results of the scoping knowledge sources

\subsubsection{Scoping Process}

\section{Selecting Sources}

This initial step searched for the relevant sources about crowdsourcing. Following the scoping approach that highlights the comprehensiveness, the search was opened to multiple knowledge databases. More precisely, it relied on eight popular bibliographic databases: ACM, EBSCOHost, Emerald, IEEE, Sage, Science Direct, Springer Link, and Wiley. Between September and November 2013, we searched for papers using the following keywords 'crowdsourcing', 'crowdsource', 'crowdsourced', 'crowdsourcer', and 'crowdsources' (the keyword 'crowdsourc*' was used to replace all the 
aforementioned keywords in certain allowable databases like Emerald, IEEE, and Sage). This choice of keywords was based on the perception that they are representative and have been popularly used by other reviewers in the field (Estellés-Arolas \& GonzálezLadrón-de-Guevara, 2012; Hossain et al., 2015). As a result, we identified 877 knowledge sources, consisting of 667 conference papers and 210 journal articles available in full text. The search results are represented in the top parts of Figure 4.1 and detailed in Table 4.1.

Table 4.1. Crowdsourcing sources on the eight bibliographic databases

\begin{tabular}{|l|r|r|r|}
\hline Bibliographic databases & Conference papers & Journal articles & Total no. of sources \\
\hline ACM & 408 & 3 & 411 \\
\hline EBSCOHost & 0 & 6 & 6 \\
\hline Emerald & 0 & 11 & 11 \\
\hline IEEE & 170 & 47 & 217 \\
\hline Sage & 0 & 20 & 20 \\
\hline Science Direct & 0 & 53 & 53 \\
\hline Springer Link & 89 & 58 & 147 \\
\hline Wiley & 0 & 12 & 12 \\
\hline Total & $\mathbf{6 6 7}$ & $\mathbf{2 1 0}$ & $\mathbf{8 7 7}$ \\
\hline
\end{tabular}

\section{Filtering Sources}

Although hundreds of sources were identified by the keyword search, many of them were clearly irrelevant to the subject of the dissertation. Following a screening technique suggested by Okoli (2015), this step filtered out the irrelevant sources using the two following actions. First, we excluded posters, tutorials, extended abstracts, and work in progress, papers which are normally too preliminary to be considered as knowledge sources. In this process, we found 22 duplicates that were stored or indexed by more than one bibliographic databases. They were also removed from the pool. Additionally, we eliminated conference papers that had been extended and published as journal versions to prevent duplication. Second, we eliminated sources applying crowdsourcing to education, medical and behaviour research, and games with a purpose because these sources have quite a different focus compared to our organisational view. We also eliminated crowd funding sources, in which organisations raise capital for investments (Palacios et al., 2016), and thus are distinct from our BPC definition. This elimination was based on the sources' titles, keywords, and abstracts. Through the filtering steps, the list of sources was sharpened into a pool of 536 sources. 


\section{Classifying Sources}

After excluding irrelevant sources, this step included sources closely related to the research problem. To check whether a source focuses on BPC and thus keep it in the pool, we analysed the source topics. However, codifying topics was not a straightforward task as there was no complete classification frame specifically relevant to BPC. To address this challenge, we conducted a deductive and an inductive classification. In the deductive approach, we generated a list of pre-defined themes, based on the three stages of the research model (Section 2.4) and research foci suggested by Kittur et al. (2013), like workflow design, task assignment, task design, and quality control. If a source addressed one of the themes, it was kept in the pool and the list of themes was updated with new topics form the source.

The inductive approach was applied in cases where no classification schema could be found. For instance, there was no schema to classify sources related to the decision whether to crowdsource or not. In these cases, we followed the procedure described on the right-hand side of Figure 4.1. The procedure is illustrated through the following example. First, we started by scanning the pool to choose sources whose titles apparently related to the decision to crowdsource, i.e. a source entitled 'To crowdsource or not to crowdsource?'. Second, we reviewed the chosen sources for identifying the relevant keywords, terms, and themes related to the crowdsourcing decision. This formed a list of terms, which was iteratively updated. Third, every unclassified source was checked to see whether it related to the term list. If so, the source was kept in the pool and the topics addressed by the source were used to update the term list. Otherwise, the source was eliminated. As a result of this procedure, we ended up adding related sources to the reviewed pool and building a term list for the codification.

Overall, we classified 238 sources related to BPC. We noted that during the classification process there were many cases where sources would broadly refer to the list of terms but present indirect links to BPC. In these cases, a decision to include the sources rather than exclude them was made in order to keep the scoping review comprehensive. Making such decision was also a part of the 'wisdom of researchers', which suggests including diverse opinions that can latter on be collectively aggregated into stronger positions. 


\section{Data Extraction}

This step extracted data from the reviewed sources to identify building blocks, decision factors, and activities of BPC. For this purpose, we developed a coding form. To test the form, a PhD student was asked to code 20 random sources and the results were compared with the researcher's coding of the same sources. This led to small modifications of the coding form. The form codified four dimensions: general information, topic, findings, and application context. First, the first dimension was general information about the source, e.g. reference, year of publication, and whether it is a conference paper or a journal article, which is typically extracted by other reviews (Okoli \& Schabram, 2010). Second, we codified the topics using the three stages of the research model and the term list, which was iteratively updated as described in the above section. Another considered dimension was the research findings, which are necessary to understand the BPC process. A part of this dimension included whether findings can be generalised to other situations or are limited to particular contexts (Mingers, 2003). Finally, the last considered dimension codified the practical outcomes of the sources, focussing on useful recommendations about BPC establishment. We also extracted to whom the recommendations were targeted and the crowdsourcing contexts where the outcomes could be applied to. Details of the coding form is presented in Appendix C.

\section{Data Synthesis}

This step aggregated the data extracted by the coding forms. We reviewed the extracted data for building blocks, processes, decision factors, and activities that guided BPC establishment. This was a four-phase procedure. First, we analysed extracted topics and findings for these elements, which were compared and aggregated. Second, we merged the 'conceptually similar' elements. For instance, quality estimation (Baba \& Kashima, 2013) and quality control (Allahbakhsh et al., 2013) were merged. Furthermore, many elements were linked to each other, e.g. expert evaluation is a technique to ensure quality control (Allahbakhsh et al., 2013). To rationalise the relationships among them, we mapped some sub-elements into more generic ones. Finally, we synthesised the sources' recommendations that were related to particular elements. As a result, elements extracted from individual sources were synthesised and transformed into thematic elements related to BPC establishment. They are discussed in the next sections. 


\subsubsection{Results of the Scoping Review}

This section reports results from the scoping review. As a result of the previous steps, we ended up working with 238 sources related to BPC. The demographic information of these sources shows that $71 \%$ of them are conference papers and $29 \%$ are journal articles, which is consistent with the significant role of conference publications in IS and computer science (Freyne, Coyle, Smyth, \& Cunningham, 2010). The number of publications per year are presented in Figure 4.2, which shows a steady increase on the number of crowdsourcing studies published since 2008. This reflects the increasing maturity of the crowdsourcing field. This review also confirms the ad hoc nature of the crowdsourcing field as a large part of the reviewed sources (65\%) provide findings that can only be generalised to a similar situation (the bottom parts of the columns in Figure 4.2). Regarding to whom the implications of the reviewed sources are targeted to, the three most popular ones are managers, process designers, and programmers, which are essentially aligned to the three-stage model discussed in Section 2.4.

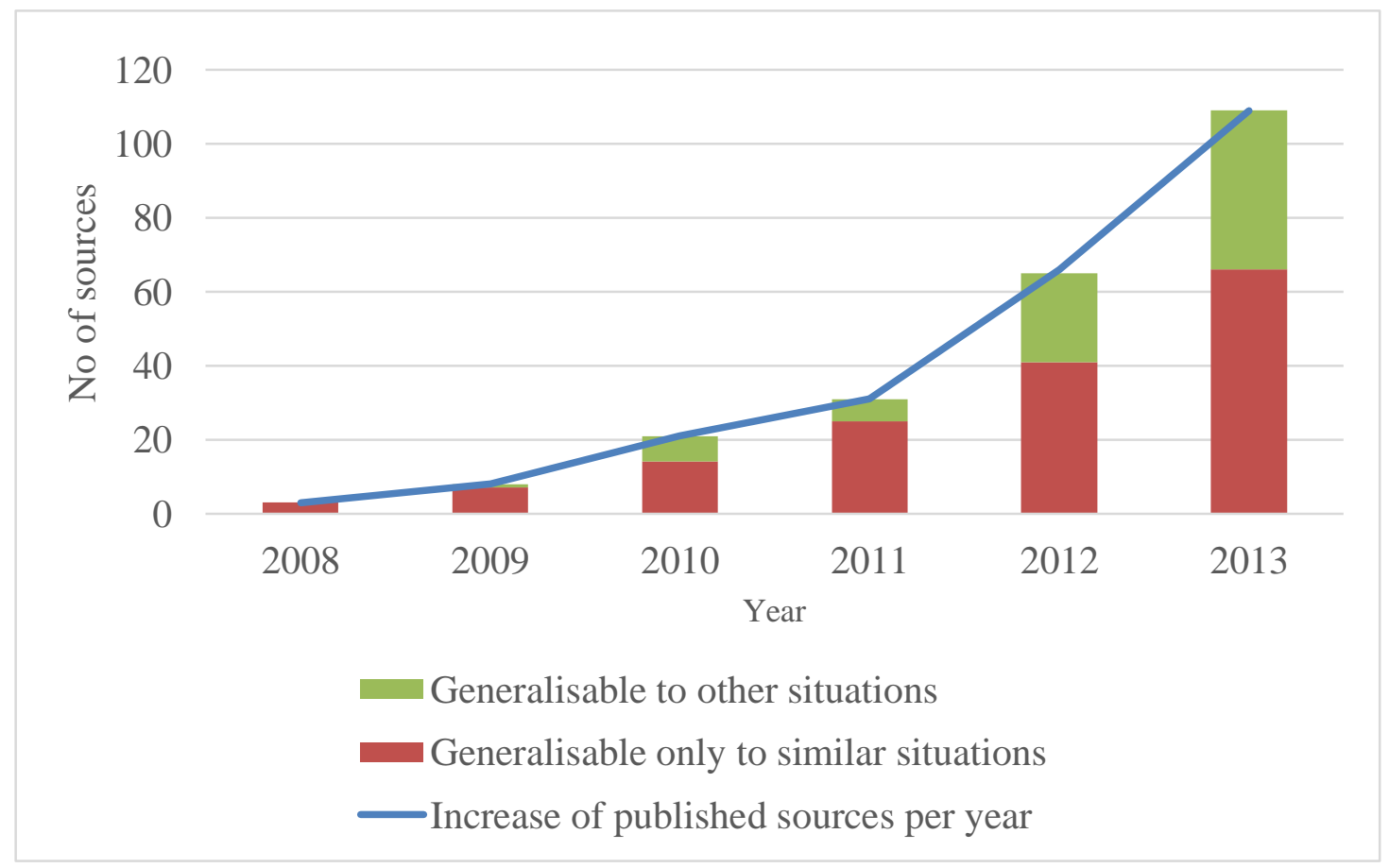

Figure 4.2. Reviewed sources per year

We now report the results of the scoping review in more detail. Considering RQ1, we analysed the reviewed sources for building blocks of BPC. 


\subsubsection{Building Blocks of BPC}

Our analysis revealed a diversity of building blocks, which are abstract elements of BPC. In particular, more than 20 building blocks and their sub-elements were identified. However, the number of sources supporting each of them was highly different. For instance, 'quality control' was supported by more than 40 sources, while some subelements like 'guide crowdsourcing with artificial intelligent' were supported by only a few sources. Based on the 'wisdom of researchers' suggesting that aggregating results from groups of researchers outperform individual ones, we refined this list by concentrating only on building blocks supported by more than a certain number of reviewed sources. Choosing this number was quite sensitive. If the number was small, we might include too many building blocks, which unnecessarily increases the complexity of the analysis at this early stage. On the other hand, if the number was large, we might include only a few building blocks and thus might not represent the domain diversity. Given that, we selected a cut-off of 10 sources to balance between representation and complexity. Another reason for choosing this value was that there was a gap between the numbers of supporting sources before (e.g. 16 sources supporting 'technical configuration') and after the cut-off value (nine and eight sources supporting 'real-time response' and 'benefit \& opportunity of crowdsourcing' respectively). As a result, Table 4.2 summarises the main BPC building blocks that are supported by at least ten sources.

Table 4.2. Main building blocks of BPC

\begin{tabular}{|l|c|}
\hline \multicolumn{1}{|c|}{ Building blocks of BPC } & $\begin{array}{c}\text { No. of supporting sources } \\
(\mathbf{n}>=\mathbf{1 0})\end{array}$ \\
\hline Quality control & 42 \\
\hline Incentive mechanism & 37 \\
\hline Crowd management & 32 \\
\hline Task design & 29 \\
\hline Result aggregation & 26 \\
\hline Workflow design & 25 \\
\hline Capability \& Characteristic of crowdsourcing & 23 \\
\hline Task assignment & 21 \\
\hline Output & 17 \\
\hline Circumstance to crowdsource \& Decision factors & 16 \\
\hline Platform & 16 \\
\hline Technical configuration & 16 \\
\hline
\end{tabular}


From this table, the most popular building block is quality control, which has been suggested by 42 sources. Quality control refers to several techniques ensuring that the "[crowdsourcing] outcome fulfils the requirements of the requester [organization]" (Allahbakhsh et al., 2013, p. 77). As crowdsourcing workers are voluntary, and thus it is hard for organisations to control their performance, quality control techniques are strongly relevant in a crowdsourcing strategy (Kittur et al., 2013; Zhao \& Zhu, 2014). Moreover, incentive mechanisms and crowd management are also popular, being suggested by 37 and 32 sources respectively. To a lesser extent, Table 4.2 also indicates other relevant building blocks of BPC and their supporting sources, such as task design, result aggregation, workflow design, etc.

Overall, all building blocks identified in Table 4.2 emerge as key elements of BPC. These building blocks indicate repeatable activities within crowdsourcing processes, which backs the BPC concept that considers crowdsourcing as a repeated business process rather than an one-off activity. Further, as the identified building blocks are salient building blocks of BPC, we suggest using them to model and structure BPC, which is the focus of the next research stage. In short, our analysis has identified a set of common building blocks, serving the basic structure of BPC.

\subsubsection{Factors Influencing the Decision to Crowdsource}

Using the same pool of knowledge sources, we also identified the important role of the decision to crowdsource in BPC establishment. This important role is partly empirical, given the building block 'circumstance to crowdsource and decision factors' in Table 4.2, and partly theoretical, based on its starting position in the BPC process (discussed in Section 2.4).

Given the important role, we further analysed factors and sub-factors influencing the decision to crowdsource. The analysis followed the aforementioned review procedure, with two extensions. First, the timeframe conducting the analysis was 2014, one year after the initial search. To keep the research up-to-date, we conducted forward searches based on the pool of sources. More precisely, we used the 'cited by' function in Google Scholar to identify the recent publications that cited the sources. The results from these searches increased the number of relevant sources on the decision to crowdsource to 50 . Second, 
the coding process was slightly modified for identifying directions of influence on the decision to crowdsource. We added quotes on the potential factors and marked ' + ' for factors that positively influence the decision and '-' for the ones that negatively influence the decision, similar to the method used by Smith et al. (2008).

Table 4.3. Factors that influence the decision to crowdsource

\begin{tabular}{|c|c|c|c|}
\hline Decision Factors & $\begin{array}{l}\text { Factor's properties/ Sub- } \\
\text { factors }\end{array}$ & $\begin{array}{c}\text { No. of } \\
\text { supporting } \\
\text { sources }\end{array}$ & $\begin{array}{l}\text { No. of non- } \\
\text { supporting } \\
\text { sources }\end{array}$ \\
\hline \multirow[t]{8}{*}{ Task } & & 30 & 1 \\
\hline & Ease of delineation & 10 & \\
\hline & Partitionable & 8 & \\
\hline & $\begin{array}{l}\text { Ease of integration with existing } \\
\text { business processes }\end{array}$ & 7 & \\
\hline & Done through the Internet & 5 & \\
\hline & Confidential information (-) & 3 & 1 \\
\hline & $\begin{array}{l}\text { High interaction or requiring } \\
\text { training (-) }\end{array}$ & 2 & \\
\hline & Hard to be automated & 1 & \\
\hline \multirow[t]{5}{*}{$\begin{array}{l}\text { Availability of the crowd } \\
\text { to perform the task }\end{array}$} & & 19 & \\
\hline & Number of members & 9 & \\
\hline & Diversity & 6 & \\
\hline & Knowledge & 5 & \\
\hline & Internet access & 3 & \\
\hline \multirow[t]{3}{*}{ Risks (-) } & & 14 & 1 \\
\hline & Low quality results (-) & 8 & \\
\hline & Loss of intellectual property (-) & 4 & 1 \\
\hline \multirow[t]{2}{*}{ Infrastructure } & & 12 & \\
\hline & $\begin{array}{l}\text { Availability of crowdsourcing } \\
\text { platform }\end{array}$ & 10 & \\
\hline $\begin{array}{l}\text { Expertise to manage the } \\
\text { crowdsourcing activity }\end{array}$ & & 6 & \\
\hline Small budget & & 4 & 4 \\
\hline \multirow[t]{3}{*}{$\begin{array}{l}\text { Lack of internal human } \\
\text { resources to accomplish } \\
\text { the task }\end{array}$} & & 3 & \\
\hline & Number of employees & 3 & \\
\hline & Knowledge & 2 & \\
\hline $\begin{array}{l}\text { Lack of internal } \\
\text { commitment (-) }\end{array}$ & & 3 & \\
\hline $\begin{array}{l}\text { Slow in technology } \\
\text { adoption (-) }\end{array}$ & & 1 & \\
\hline
\end{tabular}


Table 4.3 highlights the set of factors that influence the decision to crowdsource, and the number of sources supporting and not supporting these factors. In particular, we found nine main factors, which were then decomposed into sixteen sub-factors or properties. For each factor and sub-factor, we show the number of studies that suggest considering (or not considering) it when making a crowdsourcing decision. As seen in Table 4.3, we note that the number of supporting sources on a generic factor and the sum of the corresponding references in its sub-factors/properties may differ. The reason is that, in some cases, a source may concern several sub-factors and thus is coded multiple times, e.g. ease of delineation and partition of task (Afuah \& Tucci, 2012). In other cases, some sources study a generic factor as a whole without concerning its sub-factors, e.g. crowdsourcing task (Saxton et al., 2013).

The results from Table 4.3 indicate that 'task' is the most salient factor influencing the decision to crowdsource. $60 \%$ of the sources suggest this factor, sometimes under different names such as challenges (Seltzer \& Mahmoudi, 2013), problems (Brabham, 2008a; Muhdi et al., 2011), and crowd work (Kittur et al., 2013). This salience is because the task factor is where the substantive decision starts from. It is the first question that has to be answered when crowdsourcing (Malone et al., 2010). This factor is also important as it determines several aspects of a crowdsourcing strategy, including the targeted crowd that has the ability to perform the task, the chosen platform for publishing the type of tasks, and the internal experts supporting crowdsourcing activities. Table 4.3 also presents seven sub-factors of tasks that influence the decision to crowdsource. Four of them that positively influence the decision are whether tasks are easy to delineate (10 sources), to partition (8 sources), to integrate with existing business processes ( 7 sources), and to be done through the Internet ( 5 sources). To a lesser extent, three other sub-factors show that the decision is negatively influenced when the task includes confidential information, requires high interaction, or can be automated.

Besides the task, two factors that are most addressed by the reviewed sources are the availability of the crowd and risk. The crowd, which comprises who will perform a task, was found in $38 \%$ of the reviewed sources. These results are not surprising because the crowd is one of the three key underpinnings behind the crowdsourcing concept, as shown in Chapter 2. Out of 50,14 sources suggest the risk factor, which has a negative impact on the decision to crowdsource (i.e. more risk means less opportunity to crowdsource). 
To a lesser extent, other factors like infrastructure availability, availability of crowdsourcing experts to manage tasks, budget, internal human resources, and internal commitment also seem to influence the decision to crowdsource. Lastly, the level of organisations' technology adoption is the least addressed factor.

In summary, the review allowed us to systematically identify a set of factors that influence the decision to crowdsource. Using the identified factors, we can evaluate whether BPC is a suitable approach for a particular organisational context. Yet the relationships, similarity, and disparity among these factors still need to be examined and structured, which is the focus of the Stage 4.2 below.

\subsubsection{Summary and Remarks}

This stage conducted a scoping review of domain knowledge sources through a systematic process. The process retrieved 877 sources from eight bibliographic databases and finally considered 238 sources relevant to BPC. An overview on the reviewed sources confirmed the ad hoc nature of the BPC domain, which has supported the motivation of the dissertation to study BPC. Analysing the sources in detail, the results revealed and synthesised the major building blocks of BPC. Of them, there were twelve most salient BPC building blocks supported by at least ten reviewed sources (Table 4.2). The analysis also identified factors influencing the decision to crowdsource. It revealed nine factors and sixteen sub-factors that should be considered in the crowdsourcing decision. The identified building blocks, decision factors, and synthesised knowledge provide raw materials for the next research stages.

Overall, the scoping review offers accumulated knowledge of what the literature has reported in the domain. It has confirmed that there are repeatable processes of crowdsourcing strategies, through the identification of building blocks repeatedly suggested by the knowledge sources. The repeatable processes are the important antecedent of BPC and properly constitute business processes of crowdsourcing. Regarding the nature of the review, since the review process was arranged systematically and presented explicitly, it is possible for the review process and its results to be reproduced. This increases rigour of the review process and adds confidence to the review results. All in all, a combination of knowledge accumulation and systematic-ness constitutes the value of the scoping knowledge sources. 


\subsection{Stage 2: Conceptual Model}

Using the raw materials extracted from the knowledge sources, this stage articulated and built a conceptual model supporting the establishment of crowdsourcing as an organisational business process. Such a conceptual model had important roles in this research. The model, which articulated the raw materials into organised BPC information, provided an abstract and holistic view on the BPC domain (Cross, 1982). With its articulation, the model also underpinned the conceptualisation of BPC, and thus provided a means to explore the field. This role has been suggested by Hevner et al. (2004) that "design science research efforts may begin with simplified conceptualizations and representations of problems" (p. 85). The role of the conceptual model should also be seen as a design science outcome, where a conceptual model in design science constitutes an IS artefact per se (Hevner et al., 2004).

As the built model served as an IS artefact in design science, it should be rigorously evaluated. The current stage evaluated the model using a case study approach. More precisely, this evaluation considered the model in two crowdsourcing projects, which confirmed the adequateness and utility of the model. When considering this evaluation in the research process, the case study provided empirical evaluation of the model, which complemented the previous research efforts to conceptualise BPC.

\subsubsection{A Process Model for BPC Establishment}

To build the conceptual model, we followed guidance from Webster and Watson (2002) and Jabareen (2009) for conceptualising models from extant literature. These authors suggest that a conceptual model can be built and generalised based on a literature review. In particular, Webster and Watson (2002) suggest analysing the related literature for main concepts and processes, which are main materials for model construction. Agreeing with this suggestion, Jabareen (2009) further recommends viewing a conceptual model as not only a simple set of concepts, but rather as an organised structure where each concept plays an integral role. Following these suggestions, the current research stage used the key building blocks drawn from the scoping review, and structured them in a meaningful way. Since these building blocks were repeatable processes of crowdsourcing, this structure led us to construct a process model of BPC. 
We structured the original BPC building blocks (Table 4.2) to construct the process model of BPC. However, structuring these building blocks was not a straightforward task, since they covered very different concerns. Addressing this difficulty, the three-stage framework discussed in Section 2.4 was used as a starting point for the structuring process. We tried to allocate each building block into one of the three stages: decision to crowdsource, design, and configuration. The allocations on the decision to crowdsource and configuration were transparent, because they exhibited strong conceptual links. For instance, building blocks such as 'circumstance to crowdsource and decision factors' and 'characteristic of crowdsourcing' were logically linked to the decision to crowdsource. Similarly, 'technical configuration' was also clearly linked to the configuration activity. However, allocations of building blocks to the design activity were more difficult since the links extracted from the reviewed sources were more diffuse. To help logically organise the building blocks, we classified these building blocks into plan-time and operation-time categories according to when they are processed. The 'task design' and 'workflow design' were related with the plan-time category, as they should be done before the tasks are sent to the crowd. The remaining building blocks, including 'crowd management', 'quality control' and 'incentive mechanism' included activities that are operationalised while the crowd performs tasks. In particular, crowd management includes profiling the crowd; quality control includes identifying cheating behaviours; and incentive mechanism includes dynamic pricing, all of which process information while the crowd performs tasks. As a result, this structuring organisation led to the process model shown in Figure 4.3.

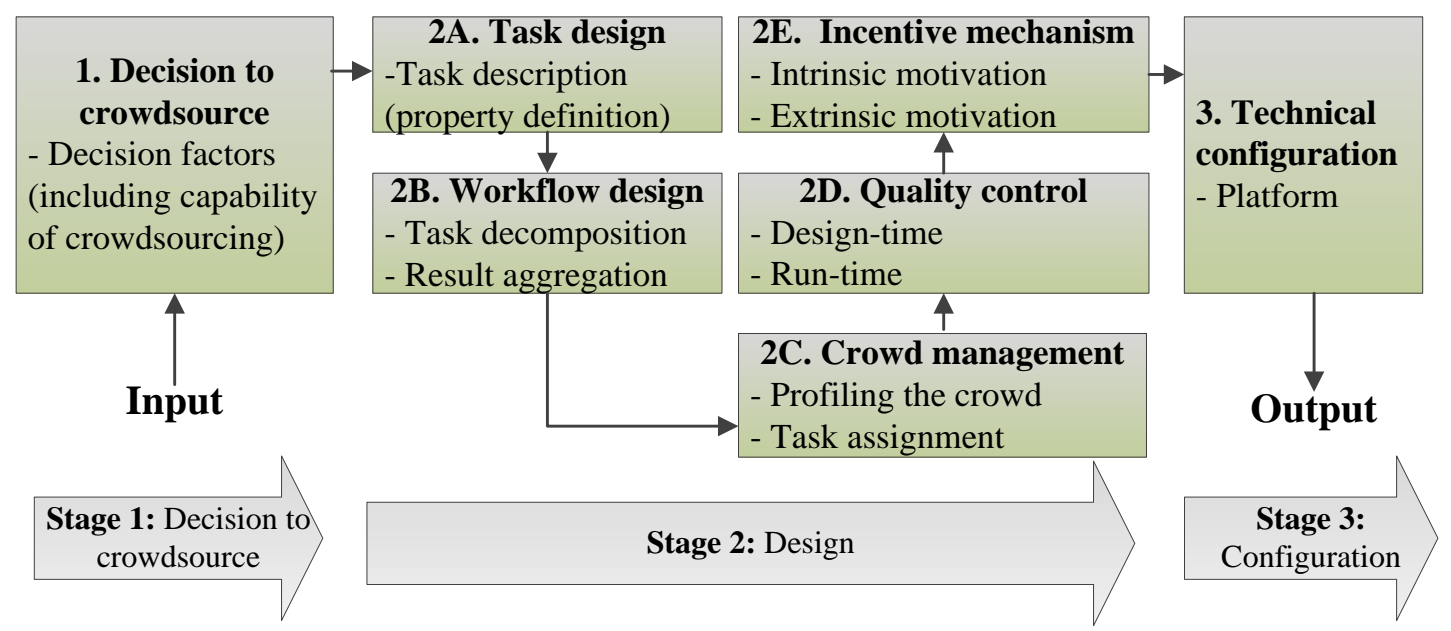

Figure 4.3. A process model for BPC establishment 
We now describe the process model in more detail. As seen in Figure 4.3, the model adopts the input-process-output (Pedersen et al., 2013) and stage-gate configurations (Cooper, 2008) that are typical of process models. It consists of seven components structured into three stages, which are described as follows.

Decision to crowdsource. The crowdsourcing process is triggered by an opportunity to crowdsource a piece of work, which starts a decision to crowdsource (Lüttgens et al., 2014; Muhdi et al., 2011; Wexler, 2011). This component initially conceptualises the crowdsourcing strategy in order to "decide whether the crowdsourcing approach is appropriate to solve their internal problem/problems [tasks]" (Muhdi et al., 2011, p. 322). It is a logical antecedent to any crowdsourcing project, aligning to a 'make or buy' decision in outsourcing projects (Dibbern et al., 2004). By making it explicit in the model, we signal that the decision to crowdsource should be founded on a logical assessment of the crowdsourcing context adequacy.

To make a logical decision to crowdsource or not, organisations need to evaluate several decisional factors. Table 4.3 has already identified several factors influencing the decision to crowdsource. However, we note that many factors in Table 4.3 may link to each other, which needs to be further arranged. Given that, we decided to structure these factors into a decision framework in order to support managers making informed decisions when they come to crowdsourcing. Yet, to keep the flow of the current section focusing on the process model and due to the important role of the decision to crowdsource, we present this framework separately in the next section.

Design. After the decision to crowdsource has been made, this stage covers a set of design activities necessary to operationalise the decision. It includes five components: task design, workflow design, crowd management, quality control, and incentive mechanism. Task design aims at transforming the conceptual ideas about the crowdsourcing tasks into a concrete task description (Model component 2A). Most of the reviewed sources recommend clearly defining the tasks that are crowdsourced (Malone et al., 2010; Rosen, 2011). The aim of this component is to designate a complete task description that can be given to the potential crowd members who may perform the tasks. To define these tasks, the properties suggested by Zheng et al. (2011) and Tokarchuk et al. (2012), like significance, autonomy, etc., should be taken into account. 
The next component concerns workflow design. This involves task decomposition and result aggregation (Model component 2B). The former decomposes the list of tasks into smaller tasks, which can often be performed with massive parallelism. This activity has been suggested by several researchers to increase the potential number of workers interested in participating in the open call (Afuah \& Tucci, 2012; Kulkarni, Can, \& Hartmann, 2012). While researchers largely agree that task decomposition is extremely useful for simple and independent tasks, several reviewed sources suggest this activity for complex and problem solving tasks (Afuah \& Tucci, 2012; Stol \& Fitzgerald, 2014). A counterpart of decomposition is result aggregation, which concerns the definition of how the outputs from the smaller tasks will be put together so that the objectives of the overall task may be fulfilled (Geiger et al., 2011). Result aggregation is closely linked to task decomposition as they are two sides of the same coin. Kittur et al. (2013) explain this relationship as a workflow that "facilitates decomposing tasks into subtasks, managing the dependencies between subtasks, and assembling the results" (p. 5).

Crowd management is a design component that refers to how organisations manage the crowd members in order to accomplish the defined tasks (Model component 2C). The reviewed sources suggest two sub-components of crowd management: profiling the crowd and assigning tasks. First, organisations analyse the required capacity of crowd members for performing a task (Allahbakhsh et al., 2012; Kittur et al., 2013), and use this evaluation to build member profiles. Based on these profiles, organisations can determine an overall picture of the crowd and may impose constraints to crowd recruitment (Chandler \& Kapelner, 2013; Stewart et al., 2010). Second, based on the crowd profiles, task assignment can be executed. That is, tasks can be assigned to crowd members who have appropriate profiles. Examples of existing task assignment mechanisms include the auction-based mechanism (Satzger et al., 2011) and the scheduled mechanism (Khazankin, Satzger, \& Dustdar, 2012b).

According to Table 4.2, quality control should be regarded as the most critical model component (Model component 2D). One distinctive characteristic of crowdsourcing is that tasks may be performed by crowd members with very different backgrounds, skills and expertise (Hirth, Hoßfeld, \& Tran-Gia, 2012). This sometimes leads to a number of low-quality contributions. Thus quality control mechanisms are critical to ensure the outputs meet the organisation's quality goals (Allahbakhsh et al., 2013; Ipeirotis et al., 
2010). By and large, quality control mechanisms can be grouped into design-time and run-time mechanisms (Allahbakhsh et al., 2013). At design-time, organisations can design tasks and workflows in a robust way, to increase the chances of receiving highquality contributions. For instance, Eickhoff and De Vries (2013) recommend that defining tasks in an unambiguous and abstract thinking way can increase quality contributions. At run-time, organisations can consider several active quality control mechanisms like expert reviews, peer reviews, gold standards, output agreements, and even peer assessments with majority voting (Allahbakhsh et al., 2013).

Crowdsourcing relies on members of the crowd voluntarily performing tasks. Thus, organisations need incentive mechanisms to attract and engage these voluntary members in their open calls (Model component 2E). The reviewed sources suggest that incentive mechanisms should be developed based on two main types of motivation: intrinsic and extrinsic. For extrinsic motivation, most of the investigated sources have examined the adoption of financial incentives (Kaufmann et al., 2011; Mason \& Watts, 2009). Regarding intrinsic motivation, a variety of factors have been suggested by the extant literature, such as fun (Doan et al., 2011), meaningful tasks (Chandler \& Kapelner, 2013), and love of the community (Kaufmann et al., 2011).

Configuration. The final component considers how to configure a crowdsourcing process for instantiation in computational systems. Since this activity mainly concerns an in-depth technical view, for instance, adopting specific architectures, frameworks, and proprietary or open computational platforms, the business perspective adopted by this study limits our considerations regarding this component. Besides, since several crowdsourcing platforms are readily available, we expect this component to be significantly constrained by the services provided by them. Furthermore, we note the extant literature has already proposed several tools supporting the configuration process. That is, we expect that in the near future, given a designed crowdsourcing process, tools may be able to automatically translate such designs into process instantiation capable of running on specific crowdsourcing platforms. Examples of such translation tools include Turkit (Little et al., 2010), Crowdforge (Kittur et al., 2011), and BPMN4Crowd (Tranquillini et al., 2015). Given that, we regard the main output of this component as a configuration file necessary for implementing the crowdsourcing process, but we do not further research the low-level details that already examined by the translation tools. 


\subsubsection{A Framework Supporting the Decision to Crowdsource}

The decision to crowdsource plays an important role in the crowdsourcing process. This role has been highlighted in the process model positioning the decision to crowdsource as the first component starting BPC (Figure 4.3). A similar role has been supported by several researchers (Lu et al., 2015; Lüttgens et al., 2014; Muhdi et al., 2011). Given the importance, researchers have proposed several factors influencing the decision to crowdsource, which have already been identified and summarised in Table 4.3.

In this section, we used the identified factors to build an analytical framework for supporting the decision to crowdsource. To this end, the 'wisdom of researchers' was applied to Table 4.3, leading to the elimination of factors suggested by only one reviewed source and focusing on factors suggested by multiple sources. We then structured the remaining factors in a meaningful manageable way. Specifically, we adapted the multilayer approach proposed by Vicente (1999), which highlights the multiple concerns that need to be understood in the decision. Consequently, we classified the decision factors in four layers, including the task, people, management, and environment. These layers are depicted as a decision framework in Figure 4.4, which is explained below.

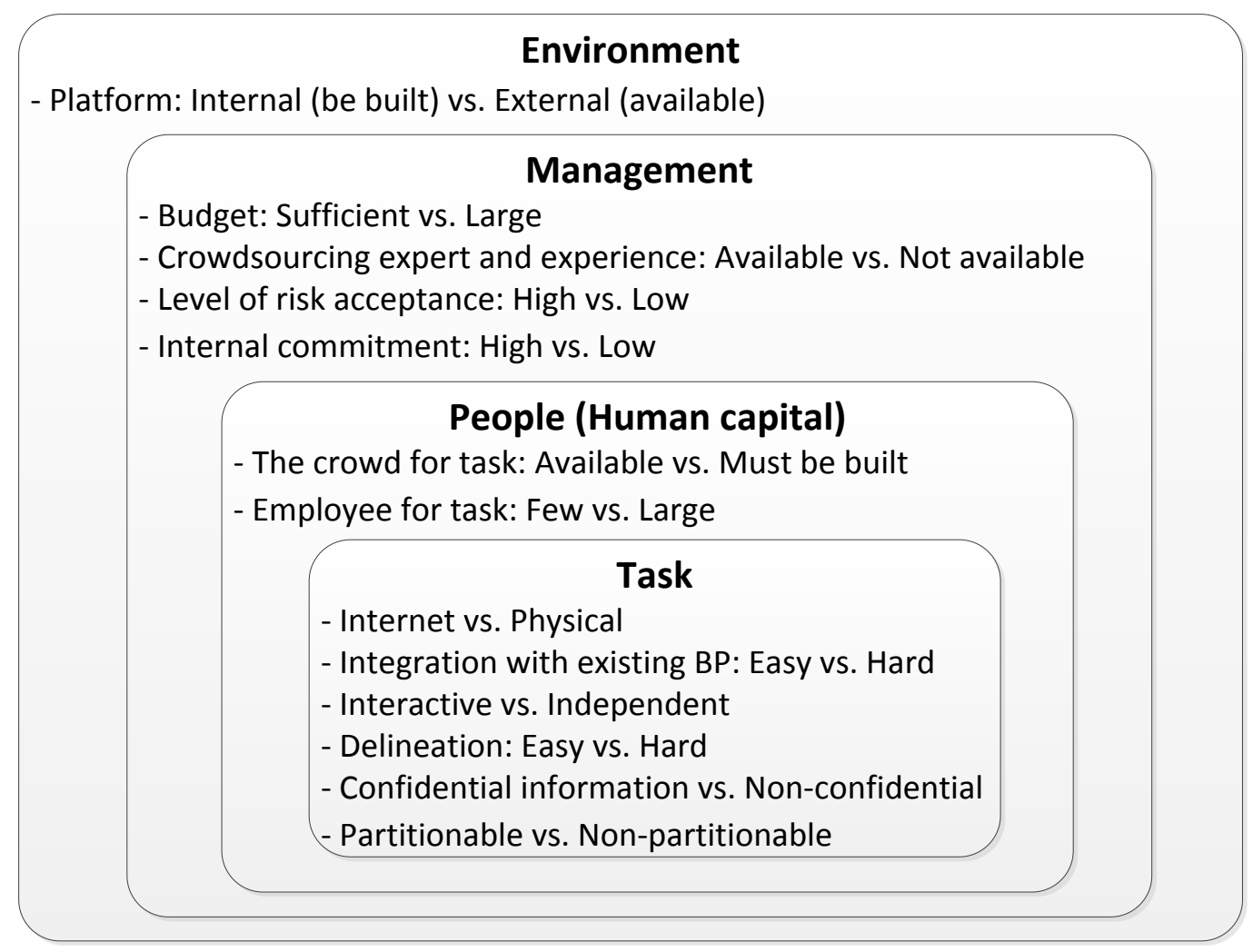

Figure 4.4. A framework that supports the decision to crowdsource 
Task Properties. According to Table 4.3, the reviewed sources suggest tasks as a key factor in the decision to crowdsource (Kazman \& Chen, 2009; Rouse, 2010; Zhao \& Zhu, 2014). From these sources, using the crowd may be good for certain tasks, but not for all kinds of tasks. Consequently, it is critical to examine task characteristics for evaluation whether an organisational task is suitable to be crowdsourced or not (Muntés-Mulero et al., 2013). This key role leads us to position this factor in the core layer of the framework. In this layer, we define six task properties.

The first property is whether a task can be performed or delivered online, i.e. its inputs/outputs can be delivered and collected through the Internet. Most of the reviewed sources consistently suggest that crowdsourcing should only be used for Internet activities (Brabham, 2008a; Doan et al., 2011; Muntés-Mulero et al., 2013). Some researchers go further adding this property to the definition of crowdsourcing, which turns this factor into one of the key underpinnings of crowdsourcing activities (Section 2.1.1). We could only find one exception in the related literature where a crowdsourcing task was not done over the Internet, but deployed through physical kiosks (Heimerl, Gawalt, Chen, Parikh, \& Hartmann, 2012).

The second property concerns the integration between crowdsourcing and the existing organisational business processes. This integration tightens and coordinates the external tasks and internal business processes (Tranquillini et al., 2015), which is strongly aligned with the BPC perspective of the dissertation. Furthermore, the important role of this factor is supported by several reviewed sources, which suggest examining not only individualised crowdsourcing tasks but the whole business process (Kittur et al., 2013; Sakamoto, Tanaka, Yu, \& Nickerson, 2011). The importance of this factor has increased recently due to the increasing adoption of crowdsourcing for complex organisational processes, including product development processes (Djelassi \& Decoopman, 2013), industrial problems (Muntés-Mulero et al., 2013), and software development processes (LaToza \& Hoek, 2016; Stol \& Fitzgerald, 2014).

Interaction is the third considered property, which focuses on the ties between the organisation and the crowd members during crowdsourcing activities. Overall, a decision to crowdsource seems unsuitable for interactive tasks that require frequent exchanges between the organisation and the crowd, or between members of the crowd (Burger- 
Helmchen \& Pénin, 2010). The reason is that it is quite hard to promote interaction when the crowd members are anonymous agents (Afuah \& Tucci, 2012). Similarly, MuntésMulero et al. (2013) also suggest avoiding crowdsourcing if complex training is required to fulfil a task. As a result, independent tasks that do not require a lot of interaction and training to be accomplished are more compatible to crowdsource.

Ten out of fifty reviewed sources highlight the fourth property, 'ease of delineation', in the decision to crowdsource (Table 4.3), which considers how the task is defined and scoped. Zogaj et al. (2014), Seltzer and Mahmoudi (2013), and Lloret et al. (2012) all suggest the positive influence of this property on the decision to crowdsource. More precisely, organisations should adopt a crowdsourcing strategy when they have welldefined and clearly-scoped tasks. The ease of delineation helps maximise the potential number of workers by increasing the crowd's understanding and so improve their approach to the task (Afuah \& Tucci, 2012). It is worth noting that task delineation may have different levels of detail, according to different stages of the crowdsourcing process, from highly abstract in the decision to crowdsource to more specific in the design and configuration.

The fifth property is whether or not tasks include confidential information, which could result in privacy and security issues. Since crowdsourcing tasks are usually sent to anonymous members of the crowd, Muntés-Mulero et al. (2013) argue that tasks with confidential information are not suitable for crowdsourcing. In a similar vein, BurgerHelmchen and Pénin (2010) suggest that the decision to crowdsource should only be made if intellectual property rights can be clearly defined. Although agreeing with the suggestion, other researchers believe that additional efforts may deal with and mitigate the problem of sensitive information. Lu et al. (2015) and Feller et al. (2012) suggest decomposing tasks into a large number of smaller tasks to conceal the overall picture, which decreases the likelihood of privacy breaches and claims regarding intellectual property.

The sixth and final property is the ease with which a task can be partitioned into smaller pieces of work. The influence of this property on the decision to crowdsource is suggested by several reviewed sources. Malone et al. (2010), when discussing the collective intelligence of the crowd, point out that a crowdsourcing strategy is more adequate for 
tasks that can be partitioned. Similarly, Afuah and Tucci (2012), regarding problemsolving tasks, hypothesise that this property positively influences probability of choosing a crowdsourcing strategy. Furthermore, this property indirectly affects the decision to crowdsource through strengthening the other aforementioned properties. Partitionable tasks are expected to be easier to delineate (Feller et al., 2012) and to protect sensitive information ( $\mathrm{Lu}$ et al., 2015), each of which positively influences the decision to crowdsource.

People. When making the decision to crowdsource, an organisation should consider the role of human capital playing in the crowdsourcing process, in terms of the crowd members and internal human resources (Afuah \& Tucci, 2012). The availability of the crowd members to perform tasks is the key factor deciding the choice of crowdsourcing as tasks in the crowdsourcing strategy are processed by the crowd members. In general, Djelassi and Decoopman (2013) and Doan et al. (2011) suggest that the high availability of members increases the possibility of adopting a crowdsourcing strategy. Afuah and Tucci (2012), examining crowdsourcing contests, identify a similar positive influence.

The availability of the crowd should be further considered through four sub-factors: the number of members in the crowd, Internet access, knowledge, and diversity. According to Table 4.3, the number of members and their ability to access the Internet are two determinants for crowd availability. Both Malone et al. (2010) and Marjanovic et al. (2012) indicate that the chance of an organisation choosing to crowdsource increases when there is a large pool of people to procure for the task. The requirement of Internet access within the targeted crowd is related to the fact that almost all crowdsourcing tasks are performed through the Internet. Consequently, Internet access influences the number of members available for crowdsourcing tasks (Brabham, 2008a; Saxton et al., 2013), and thus affects the decision whether to crowdsource or not. The other two sub-factors, i.e. knowledge and diversity, also play an important role in the crowd availability. Yet, their roles seem to depend on the nature of the task. For instance, some tasks, like software development (Stol \& Fitzgerald, 2014), require a certain type of knowledge from the crowd members, while others, such as solving a generic problem or innovation (Boudreau \& Lakhani, 2013), need a crowd with diverse backgrounds. In summary, the decision to crowdsource is influenced by "the constant availability of sufficient quantity and quality [knowledge and/or diversity] of online workers" (Corney et al., 2010, p. 244). 
The reviewed sources also suggest considering the availability of internal employees when making the decision to crowdsource. If an organisation has too few internal employees in comparison to large human resources required for the task, choosing crowdsourcing to fulfil the human resource gap is suggested (Malone et al., 2010). Lu et al. (2015) go further to explain this decision in terms of both number of employees and their knowledge for tasks. With some tasks, like image tagging and translation, requiring a huge number of human resources that often exceed an organisation's capability, crowdsourcing is a good (if not the only) option. Agreeing with the suggestion, Afuah and Tucci (2012) further considered the internal human resources regarding whether the knowledge meets the requirements for tasks. Consequently, they recommend using crowdsourcing if "the knowledge required to solve the problem falls outside the focal agent's knowledge neighbourhood" (Afuah \& Tucci, 2012, p. 369).

To sum up, the framework suggests that both high availability of the crowd and scarcity of internal employees for the tasks increase the possibility to choose crowdsourcing. When comparing the two factors, the availability of the crowd should receive higher priority. The reason is that the crowd is one key underpinning of crowdsourcing (Section 2.1.1), which is again highlighted here by many review sources, i.e. nineteen out of fifty sources in the reviewed pool, compared to three sources suggesting the role of scarce internal employees. Furthermore, though organisations may have enough internal employees for tasks, crowdsourcing is still a good approach that can bring competitive advantages for the organisations, e.g. increasing customer relationship. This can be inferred from many existing crowdsourcing projects promoted by well-resourced organisations, like Westpac bank (Westpac, 2013).

Management. Whether to crowdsource or not is a complex decision, which can influence the success of the whole project. Thus, it has to receive major attention from managers (Djelassi \& Decoopman, 2013). From a managerial perspective, Rouse (2010) advises that the decision to crowdsource should only be made after examining costs, coordination, and risks. Recent studies additionally suggest that employees' commitment is another factor influencing the decision to crowdsource (Lüttgens et al., 2014; Simula, 2013). Consequently, the management layer in our framework focuses on four factors: the project budget, the availability of expertise to coordinate the crowdsourcing activity, risks, and internal employees' commitment. 
When evaluating whether crowdsourcing is a suitable strategy, it is important to compare its efficiency in realising organisational goals in comparison with other alternatives. As cost saving is a key criterion for measuring efficiency (Muhdi et al., 2011), the budget of a crowdsourcing project influences the decision to crowdsource. Although there is a high agreement on the important role of budget in the decision, the reviewed sources seem to disagree on how this factor influences the decision to crowdsource. As seen via Table 4.3, four sources suggest a low budget, whereas an equal number of sources suggest a reasonable budget before making the crowdsourcing decision. In particular, some sources support that crowdsourcing is a preferred option when a project does not have enough money to hire new employees, or is a small-budget project (Malone et al., 2010). Whereas, others argue that a reasonable budget is required because though the amount of money to pay the crowd may be small, other costs, like coordination and transaction costs, may increase ( $\mathrm{Lu}$ et al., 2015). Although further studies are needed to solve this disagreement, we suggest that the decision to crowdsource should be made based on having sufficient budget. That is, the budget is not enough to perform tasks in the traditional way, i.e. internal sources and outsourcing, but is sufficient to cover the crowdsourcing process.

Another considered factor in this layer is whether organisations allocate appropriate expertise and experience to coordinate multiple activities of crowdsourcing. This factor greatly influences the success of crowdsourcing, as stated by Muhdi et al. (2011) that at the beginning of a crowdsourcing project, "a source of experience and expertise in crowdsourcing can be helpful to match company expectations and the realistic possibilities of crowdsourcing" (p. 323). As Rouse (2010) suggests, a lack of coordination can lead to a drain of resources and substantial delays.

By analysing the reviewed sources, we have identified a few risks that should be considered when deciding to crowdsource. According to Table 4.3, the most salient ones are the risks of low quality results (Kannangara \& Uguccioni, 2013; Naroditskiy et al., 2013) and loss of intellectual property (Schenk \& Guittard, 2011). In crowdsourcing where tasks are performed by voluntary crowd members, organisations have little control over members' behaviour (Zhao \& Zhu, 2014), and this could lead to poor contributions to the project. As a result, the risk of low quality results should be considered. Another risk is the loss of intellectual property (Marjanovic et al., 2012), which mainly links to 
skilled tasks. When relying on the crowd members for these types of tasks, organisational knowledge may have to be transferred to them (Afuah \& Tucci, 2012) and after the tasks are accomplished, knowledge related to the task may remain in the crowd. This implies the risk of losing intellectual property. Burger-Helmchen and Pénin (2010) claim that crowdsourcing should only be seen as a viable option if intellectual property can be managed and controlled. We further note that managing intellectual property is not only about hiding sensitive information, as mentioned in the task layer, but can be extended to other mechanisms, such as patents (Burger-Helmchen \& Pénin, 2010) and intermediary platforms (Feller et al., 2012). In summary, organisations have more chance of making the decision to crowdsource if they can accept and manage the two aforementioned risks. The fourth and final factor we consider in this layer is the organisational employees' commitment to crowdsourcing activities, a concern suggested by recent studies (Lüttgens et al., 2014; Simula, 2013). This factor refers to the conflicting interests of employees and managers regarding the crowdsourcing activity, which relates to overcome the issue of the 'not invented here syndrome' (Katz \& Allen, 1982). Although only a few articles in crowdsourcing literature consider this factor, we believe it is an important managerial concern because limited organisational employees' commitment "can jeopardise the success of an entire crowdsourcing project" (Muhdi et al., 2011, p. 322). This factor is further important as several tasks in a crowdsourcing project, such as task definition and workflow design, are performed internally by organisational employees and managers (Whitla, 2009; Zhao \& Zhu, 2014). As a result, a lack of employees' commitment may decrease the ability to choose crowdsourcing (Lüttgens et al., 2014).

Environment. The primary factor in this layer is the choice over the use of either internal or external crowdsourcing platforms. In terms of cost, using an external platform saves development cost, which makes the decision to crowdsource more competitive. From a resource-based view, Lu et al. (2015) support this argument by clearly specifying that "decisions on the use of online microsourcing [crowdsourcing] will be driven by the ability of online sourcing platforms to provide cheap service solutions, complement current resources, fill a resource gap, and to give access to a large pool of resources" (p. 4). Some other reasons to adopt external platforms include the large and varied pools of members, the speed of launching the crowdsourcing project, and in some cases, protecting intellectual property (Feller et al., 2012; Mason \& Suri, 2012; Zogaj et al., 2014). 
To sum up, the decision framework developed in this section has two characteristics. First, it structures the factors influencing the decision to crowdsource into the corresponding layers, of task, people, management, and environment, which are not apparent in individual sources of knowledge. Consequently, it can be used as a decision framework per se, supporting managers in their crowdsourcing decisions. Second, the framework details the first component of the process model (Figure 4.3), and thus can also be seen as an integrated plugin of the process model.

\subsubsection{Evaluation of the Model: Case Studies}

\subsubsection{Overview of the Approach}

After the construction of the process model, we decided to evaluate the model using case studies for three reasons. First, case studies allowed the model to be evaluated in the practical environments. Another reason came from the complex nature of crowdsourcing. Evaluating a model that captured such a high level of complexity required in-depth and detailed explanations about their components, links and overall structure. The capacity to discuss the model in such detail was a distinctive characteristic of case studies. These reasons were supported by Yin (2013b), who stated that "for evaluations, the ability to address the complexity and contextual conditions nevertheless establishes case study methods as a viable alternative among the other methodological choices" (p. 322). The third and final reason was that case studies are considered appropriate for evaluating design science artefacts, as suggested by Gill et al. (2015) regarding the evaluation of a social architecture framework.

For the case study evaluation, we considered the adequateness and utility of the model. We defined adequateness as 'the degree to which the components and their arrangement in the model align with the activities done in the studied crowdsourcing project', and utility as 'the usefulness of the model perceived by the crowdsourcing project managers and coordinators'. Using these two metrics, we collected and analysed data from two crowdsourcing projects. 


\subsubsection{Case Study Design}

We followed the guidelines provided by Yin (2013a, 2013b) for designing case study evaluation research, including how to select cases, collect data, analyse data, and validity.

\section{Case Selection}

The selection of crowdsourcing projects was based on comparability and access to source material. First, we selected projects with a comparable team size, between 2 and 10 members. This range of team size was sufficiently large to include multiple project roles, which the model aims to support, but not so large as to hold a diversity of settings that overshadow the evaluation purposes. Second, we chose crowdsourcing projects where we had access to project participants and other data sources. As a result, two crowdsourcing projects, Crowd Tagging (CT) and Logo Design Contest (LDC), were selected.

The CT project was part of a bigger plan aiming to uncover the impact of New Zealand predators on biodiversity in urban areas. This plan involved the installation of motiontriggered cameras in 40 locations in New Zealand, which collected more than 65.000 pictures. The CT project aimed at identifying the animals captured in these pictures. Because of the large number of pictures that needed to be analysed, the project launched a website with an open call to help tag the pictures. The project involved a team of four members: project manager, designer, web developer, and consultant. The call went live from June to December 2014. As a result, the project attracted over 300 users. About half of them tagged more than 20 pictures.

The other project, LDC, utilised the crowd for artistic design. A University in the Mekong delta, Vietnam was founded in 2013 from what began as a tertiary education centre. As a part of this transformation process, the University needed a new logo that would represent the spirit of the University. To design the logo, the University adopted a crowdsourcing approach that opened the logo design to designers from both inside and outside the University. It was in this spirit that the LDC project was created. The project started in May 2013 and finished in December 2013, when the winning logo was officially adopted by the University. The project had a leader, who made all project decisions, and a coordinator who instantiated and controlled the contest. The project also involved the University Board, consisting of eight members, who made key strategic decisions about 
the project planning. When the project was launched, it received 68 logo designs from the crowd. Three of them were selected and declared as the winning solutions: two were awarded for creative prizes and one was awarded for the final winning solution, which is the current logo of the University.

\section{Data Collection}

We collected data from multiple sources, both primary and secondary. Secondary sources included press releases, the open calls, meeting reports, and project websites, all of which provided materials necessary to clarify key project activities. The activities and their relationships were further detailed and validated in interviews. Across the two case studies, we conducted three in-depth interviews with project leaders and other participants, both face-to-face and through Skype. Due to the small size of the project teams, these interviewees wore 'many hats' and therefore could provide insights into several perspectives of the crowdsourcing projects. Besides being interviewed about the activities performed in the projects, the interviewees were asked to analyse a printed version of the model presented in Figure 4.3 and were asked to make a judgment and produce comments about the usefulness of the model. The protocol for the interview process is included as Appendix D. A summary of demographic information about the cases and their data sources is presented in Table 4.4.

Table 4.4. Demographic information

\begin{tabular}{|l|l|l|}
\hline & \multicolumn{1}{|c|}{$\begin{array}{c}\text { Crowd Tagging } \\
\text { (CT) }\end{array}$} & $\begin{array}{c}\text { Logo Design Contest } \\
\text { (LDC) }\end{array}$ \\
\hline Number of project members & 3 & 10 \\
\hline Project duration & 6 months & 7 months \\
\hline Project purpose & $\begin{array}{l}\text { To tag pictures about } \\
\text { animals in New Zealand }\end{array}$ & $\begin{array}{l}\text { To design a logo for the } \\
\text { University }\end{array}$ \\
\hline Interviews & 1 & 2 \\
\hline Roles of interviewees & - Project leader & $\begin{array}{l}\text { - Project leader } \\
\text { - Project coordinator }\end{array}$ \\
\hline Other data sources & $\begin{array}{l}\text { - Press and media } \\
\text { - Website, tutorial } \\
\text { - Internal documents } \\
\text { (e.g. example } \\
\text { submissions) }\end{array}$ & $\begin{array}{l}\text { - Press and media } \\
\text { - The open call } \\
\text { - Website } \\
\text { - Internal documents } \\
\text { (e.g. meeting reports, } \\
\text { example submissions) }\end{array}$ \\
\hline
\end{tabular}




\section{Data Analysis}

To prepare data for analysis, we first arranged a full description of each case, including details about the project, project team, and project activities. We then used the process model to map the project activities into the model components, while critically analysing the interviewees' comments about the model. More precisely, this empirical analysis included the two following activities.

Adequateness analysis: This analysis followed a pattern matching technique (Yin, 2013a). We looked for major similarities, patterns, and notable differences between the model components and the activities reported for each project. We analysed each project starting from secondary data, which included considerable information about the project activities, followed by the analysis of the interview and supplementary materials. The identified activities were finally mapped in the model for comparing the similarities and differences between them.

Utility analysis: We gathered judgements and comments from the interviewees regarding the perceived utility of the model. During the interviews, we asked evaluation questions, such as 'what do you think about the model components?' and 'what do you think about the sequence of the model components?'. Analysing answers of these questions, we then focus more on identifying patterns of 'usefulness', 'future use' and 'future improvement', rather than 'yes or no' answers as these direct answers are usually biased.

\section{Validity}

To allow judging levels of accuracy and strengthening knowledge claims of the case study, we followed techniques suggested by Yin (2013a) to establish validity. Table 4.5 presents those most relevant to the case study, and summarises how we handle them.

Table 4.5. Validity of the case studies

\begin{tabular}{|l|l|}
\hline $\begin{array}{l}\text { Construct validity } \\
\text { - Use multiple sources of evidence } \\
\text { - Reports reviewed by key project members }\end{array}$ & $\begin{array}{l}\text { External validity } \\
\text { - Use literal replications to choose the } \\
\text { crowdsourcing projects }\end{array}$ \\
\hline $\begin{array}{l}\text { Internal validity } \\
\text { - Insights from the crowdsourcing literature } \\
\text { (using materials from the scoping review for } \\
\text { the model construction) }\end{array}$ & $\begin{array}{l}\text { Reliability } \\
\text { - Use case study protocol (including } \\
\text { interview protocol) } \\
\text { - Pattern matching in data analysis }\end{array}$ \\
$\begin{array}{l}\text { Develop and maintain case study } \\
\text { descriptions }\end{array}$ \\
\hline
\end{tabular}




\subsubsection{Case Study Results}

The case study results are structured according the two investigated metrics, adequateness and perceived usefulness, which are subsequently presented in this section.

\section{Adequateness of the Model}

To report on model adequateness, we graphically represent the project activities of the two cases using the model as a baseline. This highlights not only the similarities but also the differences between our model and the investigated projects. Figure 4.5 and Figure 4.6 summarise the activities of the CT project and the LDC project respectively. To increase readability, the figures represent the similarities in normal font; differences in italic font; and sub-activities in smaller font size.

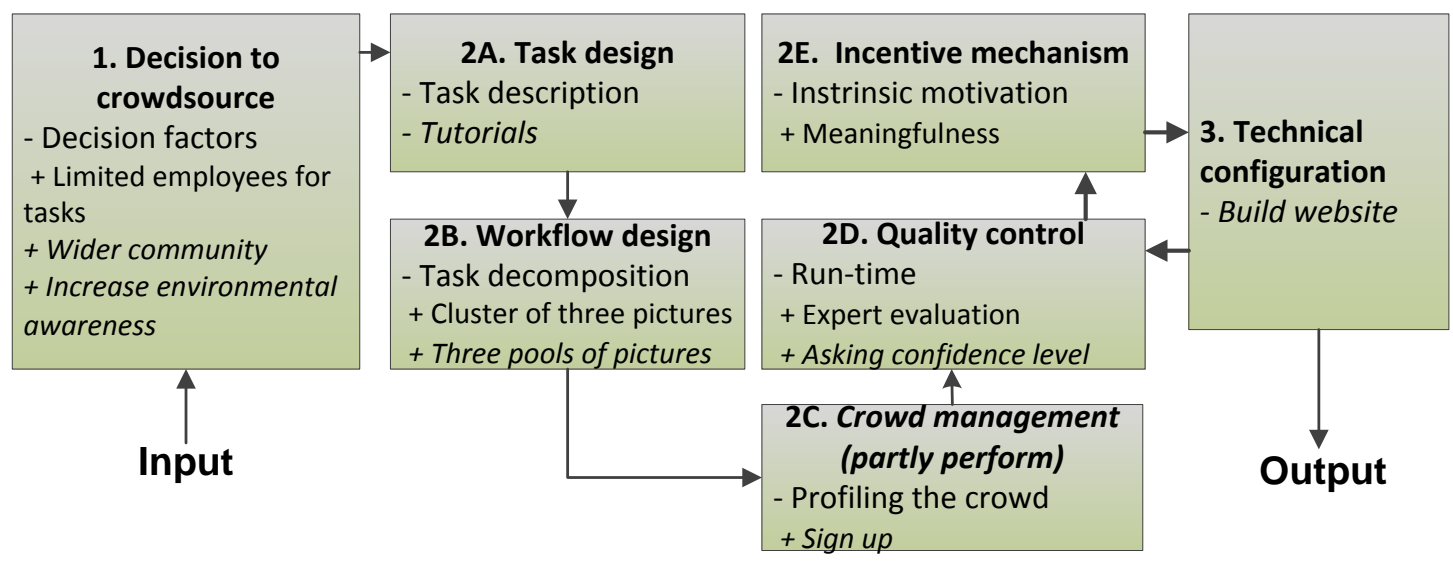

Figure 4.5. Activities of Crowd Tagging (CT)

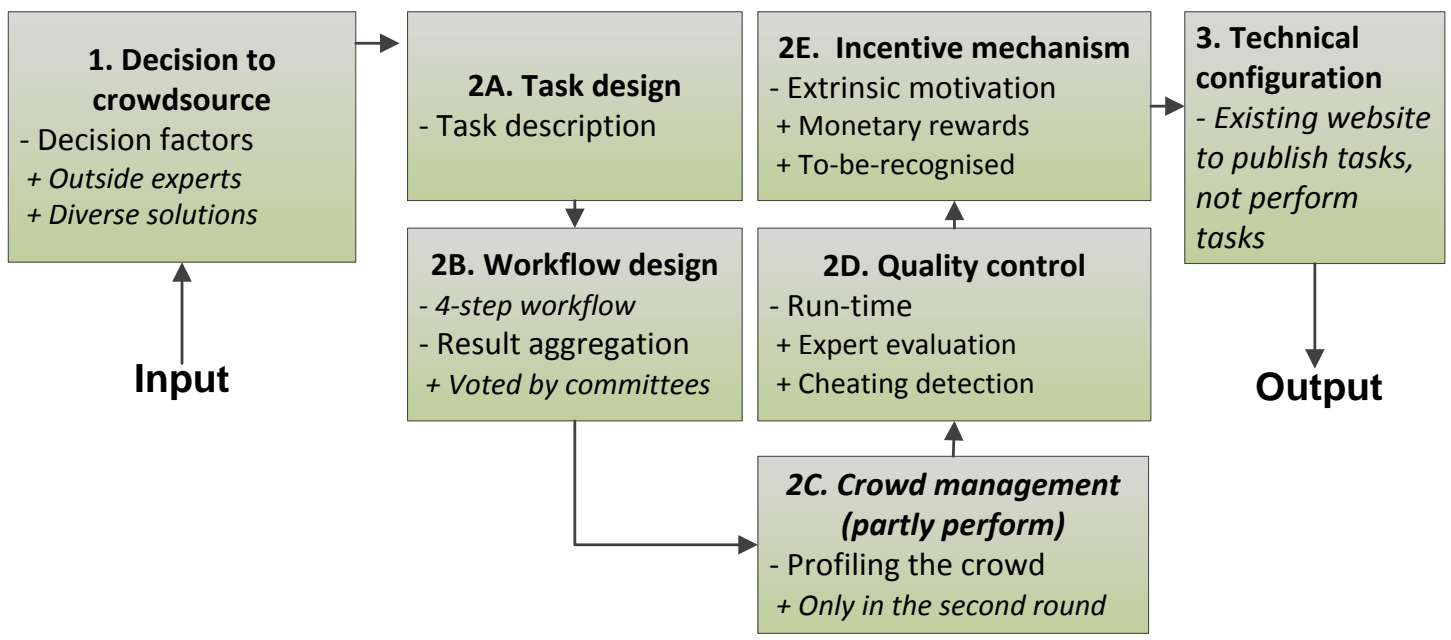

Figure 4.6. Activities of Logo Design Contest (LDC) 
Based on these graphical representations, we observe high adequateness of the model components. Both representations show strong concordance between the model components and the projects' activities. Examples include the strong alignment on the decision to crowdsource, task design, workflow design, incentive mechanism, quality control, and partial alignment on crowd management and technical configuration. Several project sub-activities are also aligned with the model. However, both cases reveal several additional (sub) activities that are necessary to instantiate the components in practice. Examples include developing a tutorial in the task design of the CT case, and aggregating results through voting in the workflow design of the LDC case. Nevertheless, we find a strong alignment between the model components and the two projects, which suggests high adequateness of the model.

Specifically regarding the interdependencies suggested by the model, the two investigated projects are also largely aligned, i.e. they generally adopt the sequence of steps from input, decision to crowdsource, several aspects of crowdsourcing design, configuration, and finally to output. This alignment is stronger in the LDC case where most components follow the model sequence. In the CT case, we find strong alignment in the first four components, but some differences in the relationships among the last three components. More precisely, the three last components of CT were developed in a more iterative way, rather than following a sequential relationship. More details about the activities and their interdependencies are presented below.

\section{Crowd Tagging $(C T)$}

The CT project started with an input consisting of a large number of pictures to be analysed. To process these pictures, the project manager decided to adopt crowdsourcing. He stated three supporting reasons: 1) limited human resources to process the vast amount of data; 2) allowing the wider community to access the collected data; and 3) increasing environmental awareness of the community. While the later reasons are specific to the nature of CT as a citizen science project, the first reason, considered as the most important factor by the project manager, is consistent with the 'decision to crowdsource' component of the process model. More precisely, we consider the lack of internal employees to perform tasks as a factor driving the decision to crowdsource (Afuah \& Tucci, 2012; Malone et al., 2010). Another reason CT should and did use crowdsourcing is the nature 
of the tasks. More precisely, tasks in CT were Internet-based; did not require interactive; were not confidential, and were partitionable. Thus, they are appropriate to crowdsource (consistent to Figure 4.4).

After deciding to crowdsource, the project manager specified the crowdsourcing process itself, starting with task design. A task description was developed to promote the general aims of the project and explain how the task could be fulfilled by the crowd: "this research aims to evaluate the use of remote cameras to estimate abundances of non-native predators in urban environments. You will be shown a series of images, taken earlier this year, from various cameras placed around the Wellington city and asked to identify the animal in the photograph" [CT, Website]. The task design is consistent with the model component $2 \mathrm{~A}$. We also note the project included a tutorial and a visual explanation of the task, which served to train the crowd on how to perform the tagging. Such focus on training seems appropriate for this type of task, and the literature suggests that training the crowd may improve the results (Park, Shoemark, \& Morency, 2014).

The CT project designed the crowdsourcing workflow through task decomposition. First, the whole activity was divided into sub-tasks of tagging three pictures, which the project alluded to as a cluster. This clustering was directly related to how data was collected in the project: "the camera takes three pictures every time they detect something. Thus, the group of three pictures helps make the task easier to perform" [CT, Project manager]. The project also divided the whole set of pictures into three pools: sign-up pool, working pool, and finished pool. The first pool included 20 clusters (of three related pictures), and the person who just signed up would start tagging the clusters in this pool. After a user finished ten clusters from the sign-up pool, the website would direct the user to the working pool. This pool included the remaining pictures that needed to be tagged, and thus was the main working zone. When a cluster had been tagged more than three times, it was considered finished and was moved to the finished pool. This pool stored the tagging results. While the three-pool decomposition is expected to improve reliability as seen below, we note that this decomposition can, and should, be extended for training purposes. More precisely, the first group can be used as gold standard data to give instant feedback and explanations as to why the crowd submissions may be (in)correct. By doing so, the crowd can learn and possibly provide better performance (Le, Edmonds, Hester, $\&$ Biewald, 2010). 
According to the proposed model, crowd management aims at understanding the targeted crowd, which enables the assignment of tasks to suitable individuals to improve performance (Allahbakhsh et al., 2012; Khazankin et al., 2012b). The CT project manages the crowd by collecting users' information and evaluating their confidence levels on task performance. Collecting demographic information about the users was done at sign-up, which was required before a user could perform a task. More importantly, the project also managed the confidence levels by using two methods. The first method was based on the first pool with known answers for the tagging pictures. By comparing users' tags with the known answers, "we can say how reliable the users are". [CT, Project manager]. Another method asked the users directly how confident they are about their submissions in order to manage the confidence levels.

Since tagging was performed by voluntary users, there was no guarantee that the results would be of high quality. Thus, quality control seems necessary for projects similar to CT (Allahbakhsh et al., 2013). However, the CT project seems to have been limited in its quality control, comparing to what were suggested in the BPC model. CT was mainly based on expert evaluation after receiving tags from the crowd. This approach led to two concerns. First, this evaluation will heavily depend on the opinion of evaluator, as seen via "I see what the people say and what I say" [CT, Project manager]. Another issue was the large amount of data that needed to be evaluated; and the project currently does not yet address this issue but sees it as future work.

To attract the crowd, the project manager considered both extrinsic and intrinsic incentive mechanisms. Regarding the former, the project manager initially thought about providing vouchers to a popular, local wildlife sanctuary (Zealandia). However, he finally decided not to do so as he believed the users would be keen enough to contribute to the citizen science project anyway. As a result, the project was mainly based on intrinsic incentives. Similar to other citizen science projects (Brabham, 2012b), this project suggests meaningfulness as an altruistic contribution to science, as stated in the website "every image you tag will help us to better understand the relationships between New Zealand's invasive mammals and native species".

In its technical configuration, CT built a crowdsourcing website that allows broadcasting the open call. This website also functioned as a platform, which enabled users to tag the 
pictures. CT decided to build its own website, rather than using some existing platforms, since the project members wanted to have full control over the whole set of crowdsourcing activities.

\section{Logo Design Contest (LDC)}

In LDC, the decision to crowdsource was based on two main factors: diverse solutions and external participants. The main reason for choosing crowdsourcing was the ability of the crowd to provide diverse and innovative solutions, as summarised by the project coordinator: "the university has decided to conduct the open contest to find ideas that are 'standard' [i.e. meeting the requirements] and creative". This is consistent with other crowdsourcing cases where external contributors can bring unique and innovative ideas (Brabham, 2010; Leimeister et al., 2009). Another factor influencing the decision to crowdsource was to utilise design contributors from outside the university. As logo design can be seen as a complex task (Schenk \& Guittard, 2011), a certain level of expertise is necessary to generate a good design. Interestingly, saving costs (compared to hiring experts) was not considered as an important factor in the decision to crowdsource.

A key activity in crowdsourcing is task design (Model component 2A). Task design in LDC was presented through the announcement that was published on the University website and the local press. This announcement included the requirements for the logo, terms and conditions to join the contest, the submission deadline, and the prizes. Within these elements, the requirements played an important role as they specified what the solution should look like (Zheng et al., 2011). This considered two aspects: meaning of the logo and technical requirements. Meaning requirements were that the designed logo should represent the spirit of the technical University. The technical requirements specified, for instance, how many pixels were needed and the length of the slogan. We noted that while the technical requirements were specific, the meaning requirements were quite abstract. On the one hand, this abstraction left plenty of room for creativity in the design solutions. However, on the other hand, it did not fully show what the University board desired about the solution, which led to an extension of the contest because of several queries for clarifying the requirements [LDC, Project Coordinator].

The workflow design was an interesting activity with two distinctive aspects. First, while the model, consistent to Afuah and Tucci (2012), suggested task decomposition, LDC did 
not crowdsource decomposed tasks, but the whole logo design. This can be explained by the nature of logo design, which could be difficult to break down into smaller tasks. Additionally, crowdsourcing a whole task has been successfully adopted for several design contests, including bus stop shelter design (Brabham, 2012b) and T-shirt design (Howe, 2006b). Second, LDC published its workflow in the open call. According the LDC announcement, the project workflow consisted of four steps: the crowd designs and submit their solutions; a preliminarily evaluation is conducted by the board; a short-list of submissions is chosen and given feedback, based on the board evaluation; and the final submissions are evaluated, ranked, and awarded. This provides transparency to the participants when explaining to them what will happen during the project.

The crowd management, which is specified in the model as task assignment and profiling the crowd, was not a focus in LDC. The project did not match the task to any specific members. Another aspect of the crowd management, which includes profiling the crowd (Allahbakhsh et al., 2012), was only processed in LDC when submissions were chosen for the second round. This was considered a limitation of LDC: "the management of crowd information was limited, which might be because we did not specify rules about providing information" [LDC, Project Coordinator]. As part of the crowd management, LDC had some communication with the contestants who wanted to find out more about the requirements. From the contest point of view, this kind of communication should be limited as it may create advantages for those contestants. Instead, a 'Q\&A' section on the website, similar to the one deployed by Threadless (2015), should have been used.

To control quality, the LDC project used expert evaluation (Zhao \& Zhu, 2014). In particular, the committee for aggregating results were also the evaluators, who assessed the submission quality and provided feedbacks. Since the number of submissions was not large (68 submissions), the use of a committee was a feasible approach. The project found a few cheating submissions that were likely copied from other logos. These submissions were mainly identified by the external experts who were experienced with logos and logo design contests [LDC, Project Coordinator].

To attract participants, the project used mainly extrinsic mechanisms, which consisted of monetary rewards and recognition by others. Like other contests, the monetary rewards were only provided for the winning solutions, which, in the LDC case, were two creative 
prizes and one final winning prize. The creative and winning prizes are quite valuable, equivalent to one and five month's salary of a typical office worker, respectively. Another motivation for the participants was that the project announced the winners on the University website, which is aligned with the to-be-recognised motivator (Brabham, $2012 b)$. Both of these motivations were clearly presented in the open call.

The technical configuration was rather simpler in this project, as LDC only used the website as a channel to publish the task and used emails to receive the submissions. This was because the project members were not aware of existing platforms/websites that can support crowdsourcing contests [LDC, Project Manager].

Overall, the results from the two cases confirm the adequateness of the proposed model to structure the project activities. Indeed, the two cases reveal a high alignment between the project activities and the model components. Adequateness is further confirmed in the interviews. The interviewees, when we show them the graphical representations of the project using the model, suggest these representations capture their projects activities. This quote evidences the suggestion: "we may miss some of the points, but we touch all of them" [CT, Project manager]. With the high adequateness, we expect that these members have a positive perceived utility of the model, as confirmed next.

\section{Perceived Utility of the Model}

Examining the perceived utility of the model, we interviewed the project members about the model, its components and sequence. The results were that all interviewees found the model to be a useful tool for structuring the crowdsourcing projects. This are demonstrated by the following comments.

"I think it will be nice to follow the model. [...]. Yes, I want to use the model, following this flow or at least have something to follow" [CT, Project manager]

"The model is very well constructed and all of its activities should be necessarily for the project" [LDC, Coordinator]

"As I said, I think this model is totally suitable. There is only slightly different on its progress, yet the meaning and purpose are similar. The approach and the steps are also similar" [LDC, Project Manager] 
Finding the usefulness of the model, these participants were extremely enthusiastic about applying the model for the future crowdsourcing projects:

"I think that any future crowdsourcing projects should apply strictly these steps, which will create better results" [LDC, Coordinator]

"From my opinion, the model can be suitable for many activities that need the resources from the crowd" [LDC, Project Manager]

In the model construction, we classified its components into plan-time and operationtime. It is interesting to find that the same idea was corroborated by a project manager. When we showed him the graphical representation of the model, he grouped the activities of the LDC project into planning and implementation, and states that:

"The component $2 \mathrm{~A}$ and $2 \mathrm{~B}$ [in the model] are similar to the planning phase of the project. The other components, including $2 \mathrm{C}, 2 \mathrm{D}, 2 \mathrm{E}$, and 3 , are implementation" [LDC, Project Manager]

These comments expressed an agreement over the perceived usefulness of the model. Furthermore, the interviewees were curious to apply the model to future projects. Interestingly, when we discussed what aspects of the model are most useful, we found slightly different views between the project manager and coordinator roles. For instance, in the LDC case, while the project manager viewed the model as a tool for making decisions and management, the project coordinator instead stressed the role of the model in supporting communication among project members and in achieving a consensus. These differences suggest that usefulness can be perceived from different angles. Through this point, we highlight that if different roles can generate different insights when using the model, then the model's utility is expanded.

In summary, we conducted two case studies evaluating the process model. The results of the case studies found strong evidence that the model can represent the key activities of crowdsourcing projects. Furthermore, we also obtained evidence of the perceived usefulness of the model, inspired by the reception of the crowdsourcing experts. Consequently, we suggest that the proposed model addresses most organisational concerns within the crowdsourcing process, and that the model can be useful to support crowdsourcing projects. 


\subsubsection{Summary and Remarks}

To guide organisations in their establishment of BPC, this stage developed a conceptual model allowing organisations to understand the main building blocks of BPC. Using the identified building blocks extracted in the previous stage, we constructed a process model of BPC consisting of seven components. The construction was based on the 'wisdom of researchers', which enabled us to build the model faithfully representing BPC. The model was evaluated using the case study approach. Two crowdsourcing projects were used for this evaluation. The results indicate that the model is adequate and useful in structuring the main crowdsourcing activities.

Overall, the model represents the main structures of BPC to support the establishment of crowdsourcing as an organisational business process. It provides a broad view of what activities need to be considered when planning, designing and instantiating crowdsourcing processes. This broad view, on the one hand, overcomes the excessive ad hoc criticism found in the crowdsourcing literature (Geiger \& Schader, 2014; Man-Ching et al., 2011). On the other hand, it represents only the abstract view but not the deconstructed view, both of which together characterise BPC. From the deconstructed view, the process model and its components need to be further analysed into detailed elements. The following stage addresses this need, which builds an ontology from both abstract and deconstructed views. 


\subsection{Stage 3: Ontology}

The purpose of this stage was to examine BPC from both abstract and deconstructed views. For this purpose, we built an ontology of BPC that captured main concepts and relationships in the domain (Corcho et al., 2003). As a result, the BPC ontology provided the ontological structure necessary to understand the main constituents of BPC. Furthermore, ontologies can enhance reasoning knowledge (Valaski et al., 2012), and thus constructing a BPC ontology made a further step towards supporting BPC establishment. Keeping the foundations of design science research, we built and then evaluated the ontology. Specifically, we built the ontology following the conceptual model and using raw knowledge materials from the previous stages. We evaluated the ontology by comparing it with an ontological version generated by software.

\subsubsection{Building a BPC Ontology}

To start the building activity, we reviewed the ontology engineering literature to identify and justify the activities of ontology construction. This led us to adopt the two activities commonly used in ontology engineering: ontology capture (Uschold \& King, 1995) and knowledge organisation (Küçük \& Arslan, 2014).

\section{The ontology capture}

This activity aimed at deriving ontological elements. We analysed the knowledge sources extracted by our scoping review for concepts, hierarchical relationships, decision-making relationships, and business rules related to crowdsourcing. This analysis was supported by the following ontology schema, which provided a structured meta-model for knowledge source analysis (Levy \& Ellis, 2006; Okoli \& Schabram, 2010). The ontology schema is graphically presented in Figure 4.7. It consisted of two main areas named ontological representation and knowledge representation, which were used to analysed the knowledge sources in both deductive and inductive approaches. 


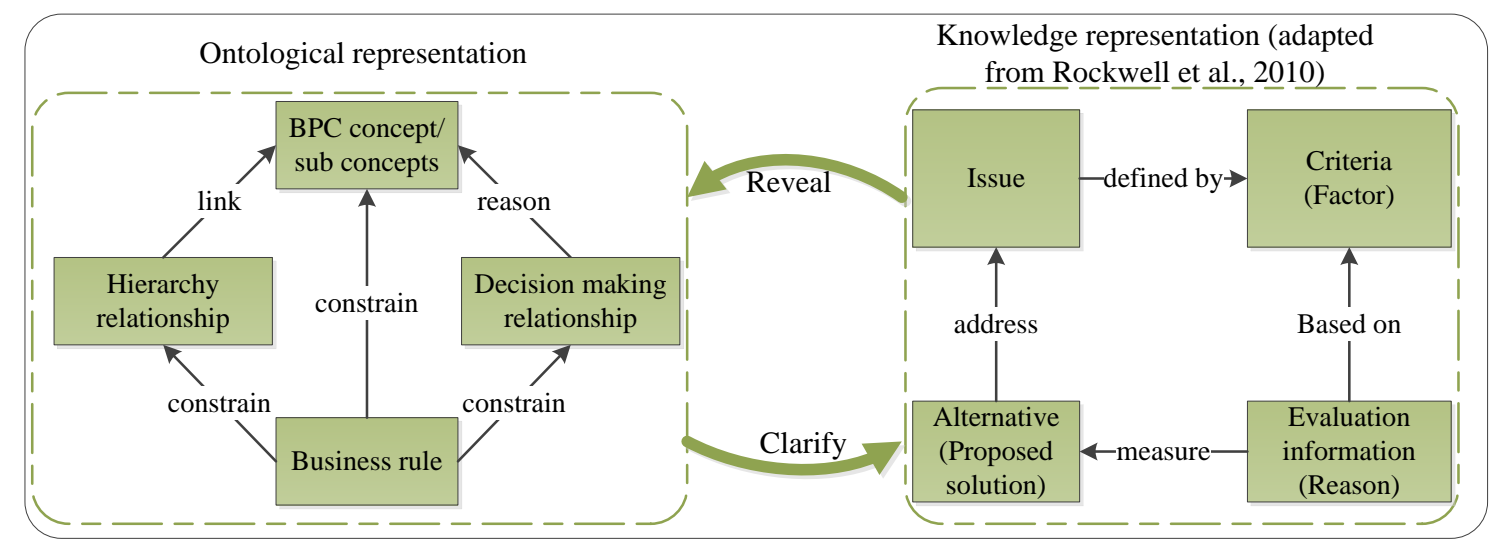

Figure 4.7. Ontology capture schema (adapted from Rockwell et al., 2010)

In the left-hand side of Figure 4.7, the ontological representation included four ontological elements: concepts, hierarchy relationships, decision-making relationships, and business rules. While concepts and hierarchy relationships were important to structure a domain knowledge (Corcho et al., 2003; López et al., 2004), decision-making relationships and business rules provided reasoning knowledge and thus were also critical when developing an enterprise system. The ontological representation was mainly used for deduction.

When deducting, we analysed the sources for concepts and sub-concepts using the components of conceptual model as the pre-defined themes. For each extracted concept/sub-concept, we specified its name, synonym, and description. Since ontologies include both the concepts and the linked extensions between concepts (Corcho et al., 2003; López et al., 2004), the next considered element was the relationship. In this element, we analysed not only hierarchical relationships but also decision-making relationships. The former referred to taxonomic structures in the domain (López et al., 2004), where we adopted five hierarchy relationships commonly used in ontology engineering, including 'is a', 'include', 'categorise', 'instance of', and 'based on'. The latter referred to reasoning relationships supporting decision-making. In this type of relationship, we chose the following ones: 'positively influence', 'negatively influence', and 'associate', which were popularly suggested in the literature (e.g. Chandler \& Kapelner, 2013; Hoßfeld et al., 2013). The last considered element concerned business rules, which added constraints to the concepts and relationships.

In the analysis, we faced an issue that some emerging elements did not align with our predefined codes. This issue is normally expected in emerging research fields like 
crowdsourcing where diverse views and methods are adopted (as discussed in Chapter 2). Addressing this issue, we used an inductive analysis that allowed studying and characterising knowledge from the bottom up. Thus, we adapted the knowledge representation approach proposed by Rockwell et al. (2010) (the right-hand side of Figure 4.7), which characterised knowledge by four categories: issue, criteria, evaluation information, and solution alternatives. Based on these categories, we applied the following questions when analysing the knowledge sources: what are the main issues related to BPC? How can these issues be defined, i.e. characterised by what factors? What alternatives can be chosen to address the issues? And how can we evaluate the proposed alternatives?

We note that the two aspects of the ontology schema (ontological representation and knowledge representation) support each other, and together assist the data analysis. While the ontological representation allows eliciting existing knowledge, the knowledge representation helps further clarify and fulfil the knowledge gaps. Another strong point of this schema is its ability to be used in both deductive and inductive approaches. Whilst the deductive analysis ensures that the captured elements align with the conceptual model and thus address core concepts of the crowdsourcing domain, the inductive analysis captures the emerging nature of the crowdsourcing field. This schema was applied to every source in our pool. As a result, we captured a large number of ontological elements: concepts, hierarchy relationships, decision-making relationships, and business rules, which are structured in the next step.

\section{The knowledge organisation}

This activity synthesised the ontological elements extracted in the previous activity and organised them to form the ultimate BPC ontology. The 'wisdom of researchers' was again applied to each ontological element: distilling concepts, hierarchy relationships, decision-making relationships, and business rules. This allowed finding salient elements that were supported by multiple sources of knowledge. We noted that during synthesis, there were several cases where the sources were inconsistent on certain extracted elements. For instance, different reviewed sources proposed different hierarchical relationships for quality control mechanisms. Some structured quality control into designtime and run-time mechanisms (Allahbakhsh et al., 2013). A slightly different 
categorisation defined before-task, during-task, and after-task mechanisms (Alonso, 2013). Other authors proposed completely different categories, including supervised and unsupervised mechanisms (Baba \& Kashima, 2013). In these cases, the 'wisdom of researchers' helped to choose the elements supported by the majority of sources.

Based on the synthesised elements, we then organised and structured them into the ontology. Since the relationships revealed the fundamental structure of the BPC domain, the ontology ended up being organised around them. It was nevertheless important to note that the organisation process was highly iterative, where we followed a trial-and-error process and updated the ontology several times. The organisation process was also performed using inductive and deductive strategies. This could be exemplified with the procedure of obtaining hierarchical relationships. In the deductive synthesis, we followed guidance from the relationships suggested by a majority of the reviewed sources, e.g. quality control can be classified into design-time and run-time mechanisms (Allahbakhsh et al., 2013; Alonso, 2013). However, no guidance was found for some groups of concepts. In these cases, we aligned with Nickerson et al. (2012) and inductively identified common characteristics of the (sub) concepts and then proposed their classification schema.

\subsubsection{An Ontology of BPC}

We now report the results from the ontology building. The section starts with the most popular concepts of BPC, and then summarises the hierarchy relationships that are used to organise the ontology. Subsequently, decision making relationships and business rules in the domain are described.

\subsubsection{Salient Concepts}

Adopting the 'wisdom of researchers', we focus on concepts suggested by multiple knowledge sources. Table 4.6 presents the 39 most salient (sub) concepts supported by at least 10 sources. At a high level, Table 4.6 represents the main building blocks of BPC, while at a more detailed level it clarifies these building blocks with their categories and sub-concepts. This clarification suggests that the conceptualisation captured in Table 4.6 has a more detailed level of abstraction compared to Table 4.2. To increase readability, 
Table 4.6 shows several building blocks of BPC in bolds, categories in italic, parent concepts in Capital-first-letters, and sub concepts and attributes in all-lower-letters.

Table 4.6. $\quad$ Salient concepts of BPC

\begin{tabular}{|l|r|}
\hline \multicolumn{1}{|c|}{ Concept } & $\begin{array}{c}\text { No. of } \\
\text { supporting } \\
\text { Sources }\end{array}$ \\
\hline Quality control & $\mathbf{6 9}$ \\
\hline Design-time & 11 \\
\hline worker selection & 16 \\
\hline Run-time & 13 \\
\hline identifying malicious behaviour & 19 \\
\hline gold standard & 16 \\
\hline output agreement & 12 \\
\hline Incentive mechanism & $\mathbf{4 6}$ \\
\hline monetary reward & 29 \\
\hline fun & 11 \\
\hline Crowdsourcing output & $\mathbf{3 8}$ \\
\hline output quality & 36 \\
\hline Task design & $\mathbf{3 7}$ \\
\hline Task description & 10 \\
\hline Crowd management & $\mathbf{3 4}$ \\
\hline Task assignment & 20 \\
\hline Profiling the crowd & 10 \\
\hline worker profile & 10 \\
\hline worker reputation & 10 \\
\hline Crowdsourcing task & $\mathbf{3 4}$ \\
\hline simple task & 13 \\
\hline complex task & 12 \\
\hline
\end{tabular}

\begin{tabular}{|l|r|}
\hline \multicolumn{1}{|c|}{ Concept } & \multicolumn{1}{|c|}{$\begin{array}{c}\text { No. of } \\
\text { supporting } \\
\text { Sources }\end{array}$} \\
\hline Decision to crowdsource & $\mathbf{2 6}$ \\
\hline Decision factor & 19 \\
\hline Task characteristic & 30 \\
\hline ease of task delineation & 13 \\
\hline partitioned task & 11 \\
\hline Availability of the crowd & 19 \\
\hline Risk \& Challenge & 16 \\
\hline \begin{tabular}{l|r|} 
Availability of crowdsourcing \\
platform
\end{tabular} & 10 \\
\hline Characteristics of the crowd & $\mathbf{2 3}$ \\
\hline Type of worker & 12 \\
\hline Motivation of the crowd & 10 \\
\hline Workflow design & $\mathbf{2 1}$ \\
\hline Result aggregation & 29 \\
\hline Task decomposition & 10 \\
\hline Control and feedback & $\mathbf{1 7}$ \\
\hline Technical configuration & $\mathbf{1 4}$ \\
\hline Platform (intermediary) & 13 \\
\hline Name conventions & \\
Building blocks are in Bold; categories are in \\
italic; parent concepts are in
\end{tabular}

In Table 4.6, the concepts were supported by at least ten knowledge sources, which indicated that they were consensually salient in the BPC domain. Thus, they were treated as the core elements of the ontology. Besides these core elements, we noted that other concepts were still considered in the ontology construction. The reason was that the importance of a concept should be indicated by not only the number of supporting sources but the relationships with other concepts, given the important roles of relationships in ontologies (Guarino, Oberle, \& Staab, 2009; Sánchez \& Moreno, 2008). A dictionary explicitly defining these concepts is provided in Appendix E 


\subsubsection{Hierarchy Relationships}

Using the concepts identified in the previous section, we organised them hierarchically to give a more holistic picture on the BPC domain. Our analysis disclosed a diverse hierarchical structure of BPC, which can be seen by counting each type of relationship: 'include' (78 sources), 'instance of' (30 sources), 'categorise' (22 sources), 'based on' (22 sources), and 'is a' (19 sources). To organise the identified relationships and concepts in a manageable way, we followed a trial-and-error process, in which we tried different structures, including a tree structure, a network structure, and a layer structure. Yet, the first two structures did not appear to be suitable to our goals. The tree structure consisted of several branches with division concerns, and thus was different from the holistic view of BPC. While supporting a holistic view, the network structure made the ontology representation too complex, with many links and crosscuts.

As a result, we adopt the layered structure, which diminishes complexity by arranging concepts and relationships into layers. Furthermore, the layered view is appropriate for integrating crowdsourcing into the organisation as integration is usually done at different levels (layers) of concerns (Giachetti, 2004; Hasselbring, 2000). The layered structure is presented in Figure 4.8. In this figure, the three kinds of relationships that are represented are 'include', 'categorise', and 'based on'. We note that the 'is a' relationship has been transferred to 'include' and 'categorise' relationships, and the 'instance of' relationships are not shown in order to reduce the complexity and to increase readability of the figure.

Figure 4.8 represents BPC main concepts and hierarchical relationships of BPC, which according to Corcho et al. (2003) captures a lightweight ontology of BPC. The ontology is organised in four layers with an increasing level of detail from inner to outer. At the heart of the framework, the core layer represents main concerns that should be focused in order to establish BPC. This layer captures the most abstract building blocks of BPC, which are aligned with the conceptual model (Section 4.2). To clarify these building blocks, the other concepts identified in Table 4.6 are further organised. They are presented in the next layers as: process layer, data layer, and data attribute layer, which is one typical schema for classifying IS objects (Giachetti, 2004; Zachman, 1987). 


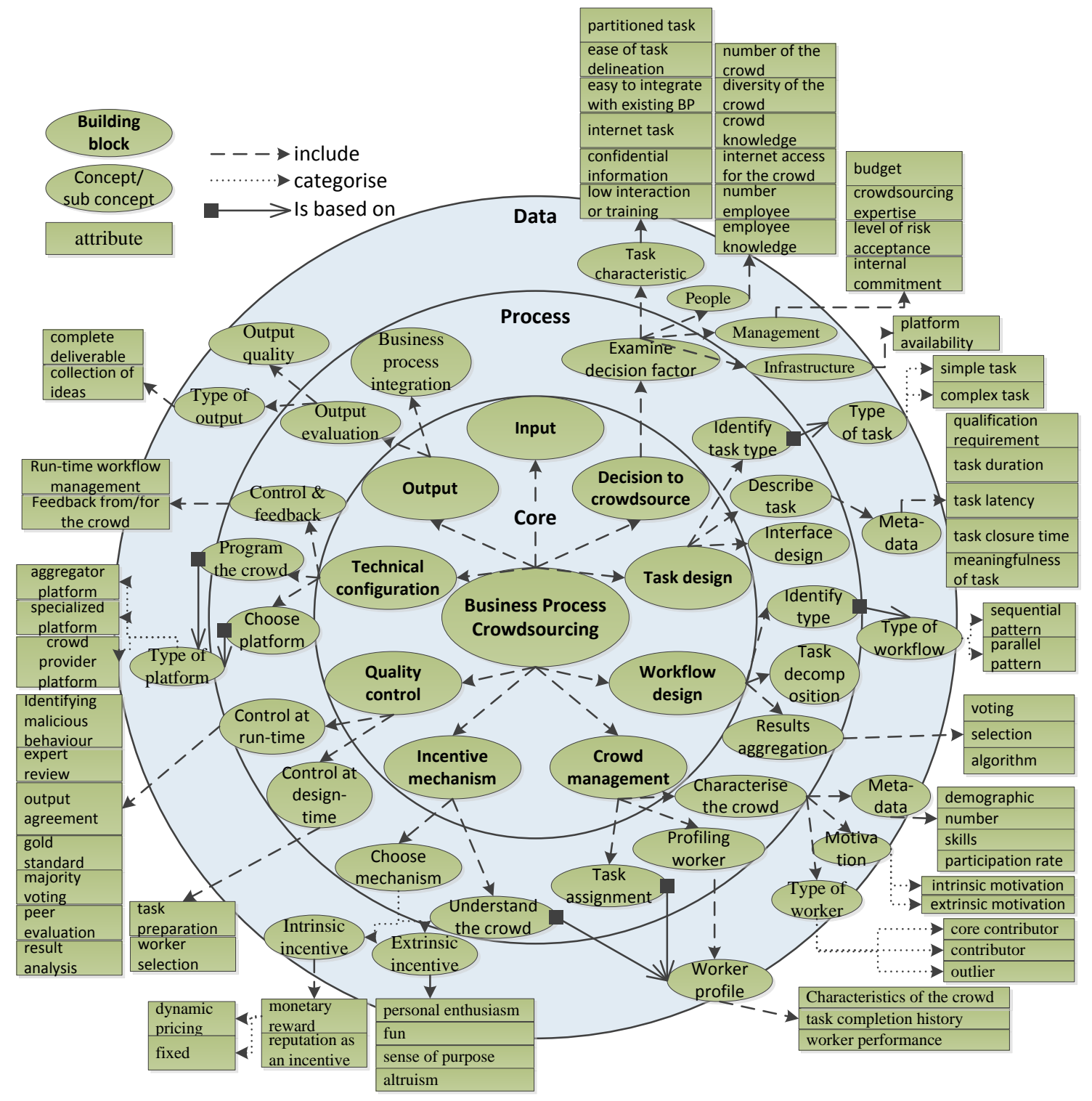

\section{Figure 4.8. A lightweight ontology of BPC}

As seen in Figure 4.8, the process layer describes plans of action that are performed in particular building blocks. In other words, the layer details each building block through a set of activities addressing a particular concern in BPC establishment. As a result, the process layer links with the core layer mainly through the 'include' relationship. For instance, the workflow design includes three activities: identifying type of task (Dai, Lin, \& Weld, 2013), task decomposition, and results aggregation (Kittur et al., 2013). From an IS perspective, most processes/activities require certain information and data to be used (Berente, Vandenbosch, \& Aubert, 2009), which suggests a strong link between this layer and the next data and data attribute layers. 
The next layer is the data layer that shows data entities and information used by the activities. For instance, to examine factors of the decision to crowdsource, decision makers need to process four data entities about task characteristics, people, management, and infrastructure, as already discussed in Section 4.2.1.1. Regarding the relationships between the process and data layers, the data usage is denoted through two main types of relationships: 'include' and 'based on'. Some activities clarify (or include) data entities, e.g. 'characterising the crowd' includes clarification of 'meta-data'. Other activities are founded on pre-defined data, e.g. the activity 'identify type of workflow' is based on data about 'type of workflow'.

The outermost layer then represents the attributes of each data entity. This representation is expressed through 'include' and 'categorise' relationships. The 'include' relationship shows that an attribute is a part of the data entity. For example, the meta-data for task description includes qualification requirement, task duration (Chilton, Horton, Miller, \& Azenkot, 2010), and may also consists of other attributes. Also presenting the whole-part relationship, the 'categorise' relationship further requires that all of the attributes make up an exhaustive decomposition of the whole concept. For instance, the reviewed sources suggest three categories of workers: core contributor, contributor, and outlier (Chanal \& Caron-Fasan, 2010; Stewart et al., 2010).

From the hierarchical relationships to structure the lightweight ontology, we note here two interesting points. First, these relationships enable explicitly structuring the related (sub) concepts in the domain. For instance, Figure 4.8 shows that quality control can be managed at both design-time and run-time, each of which includes several detailed mechanisms for quality control management. Second, by ontologically structuring these relationships, some interesting links that were not revealed by individual sources are shown in Figure 4.8. The connection between incentive mechanism and crowd management can be seen as an example. In particular, organisations should understand the targeted workers when designing incentive mechanism (Chanal \& Caron-Fasan, 2010). This understanding can be achieved through worker profiles built by the 'crowd management' building block (Khazankin, Psaier, Schall, \& Dustdar, 2011). This suggests a close link between 'incentive mechanism' and 'crowd management', which is presented via the concept of 'understand the crowd' in Figure 4.8. 


\subsubsection{Decision Making Relationships and Business Rules}

As mentioned earlier, ontologies can be classified into lightweight ones representing structured knowledge and heavyweight ones capturing both structured and reasoning knowledge of a domain (Corcho et al., 2003; Valaski et al., 2012). For the purpose of BPC establishment, the reasoning knowledge is important as it can guide the establishment activities. Thus, this section aims to add reasoning knowledge to the lightweight ontology, thus turning it into a heavyweight ontology.

For this purpose, our analysis revealed several decision-making relationships and business rules in the BPC domain. Regarding decision-making relationships, the reviewed sources identified a number of relationships, including 89 'positive influences', 17 'negative influences', and 10 'associations'. Again, the "wisdom of researchers" was applied to choose the relationships either suggested by multiple sources or linked salient concepts. We then organised the chosen relationships based on the lightweight ontology but removed the process layer since only a few decision-making relationships could be identified in the layer. For simplification, Table 4.7 summarises the association relationships while Figure 4.9 represents the other relationships.

\section{Table 4.7. Association relationships in BPC domain}

\begin{tabular}{|l|l|l|}
\hline \multicolumn{1}{|c|}{ Concept 1 } & Relationship & \multicolumn{1}{c|}{ Concept 2 } \\
\hline Output quality & associate & $\begin{array}{l}\text { Worker profile, task design, quality control, } \\
\text { task complexity, and monetary reward }\end{array}$ \\
\hline Type of task & associate & $\begin{array}{l}\text { Type of workers, incentive mechanism, benefits } \\
\text { for organisations, task design, and result } \\
\text { aggregation }\end{array}$ \\
\hline $\begin{array}{l}\text { Incentive } \\
\text { mechanism }\end{array}$ & associate & Type of worker \\
\hline Task design & associate & Quality control \\
\hline
\end{tabular}




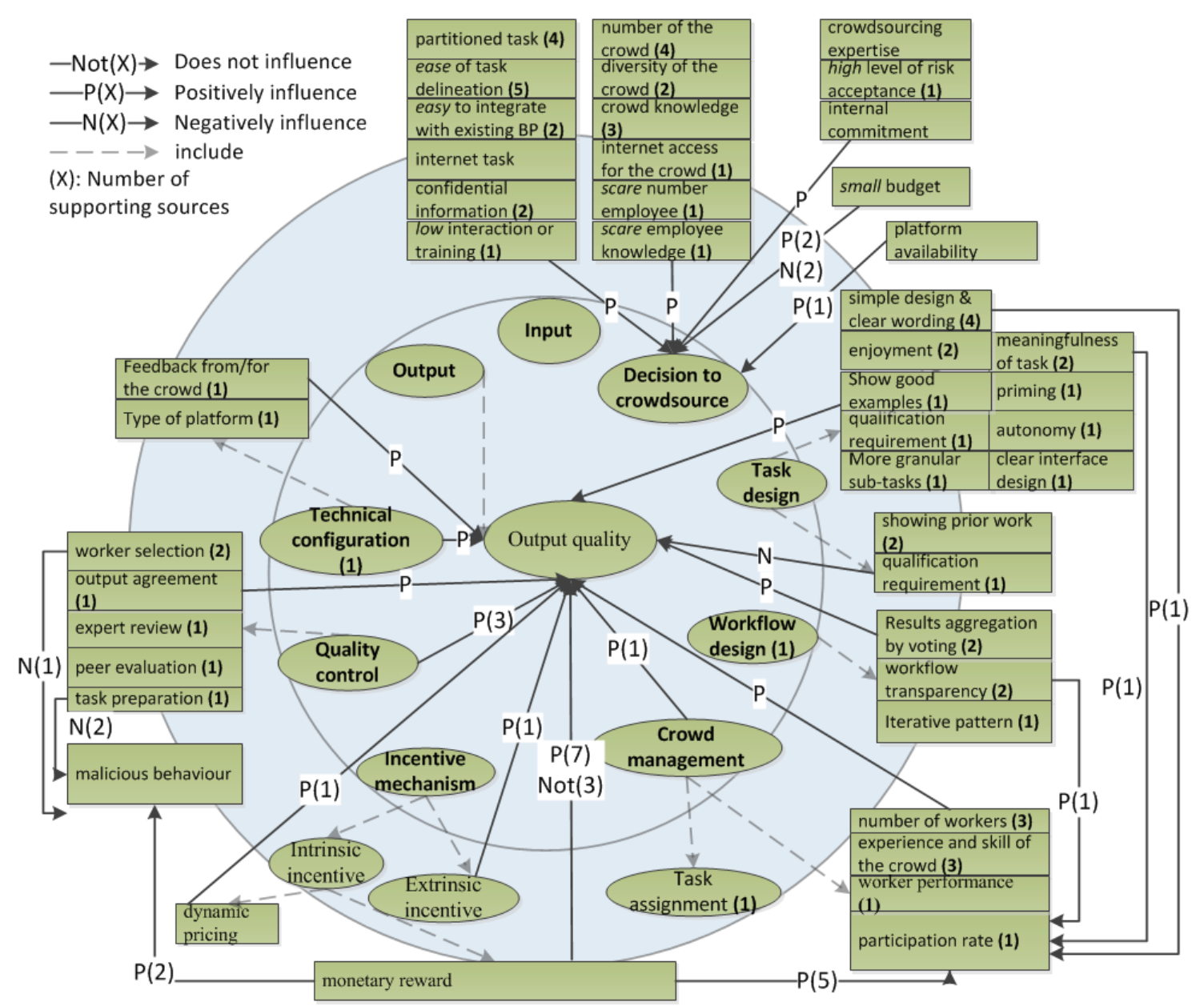

Figure 4.9. Positive and negative influence relationships

Figure 4.9 presents the key decision-making relationships in the BPC domain. Besides the 'include' relationships adopted from the lightweight ontology, there are two main relationships: positive $(\mathrm{P})$ and negative $(\mathrm{N})$ influences. The numbers, shown either on the line or behind the concept, indicate how many sources supporting a particular relationship. For instance, there are three reviewed sources suggesting that quality control positively influences output quality. In addition to the main relationships, Figure 4.9 also notes some cases where the reviewed sources do not find statistical evidence to support a particular relationship, which is presented as 'does not influence'.

As represented in Figure 4.9, our analysis reveals three important findings. First, although there are diverse relationships between different concepts, the key one is how to influence crowdsourcing 'output quality' (Archak, 2010; Chandler \& Kapelner, 2013). This is logical as 'output quality' indicates the success of crowdsourcing projects. Furthermore, a crowdsourcing project is effective only if it can achieve high quality output. As a result, we have allocated the 'output quality' concept in the centre of Figure 4.9. Second, a few 
conflicting relationships can be found, such as the influence of monetary reward on output quality. While seven sources suggest a positive influence, three other sources do not find statistically significant results to support the influence. In these cases, further research is necessary to test and confirm the relationship. Finally, we found that the number of sources supporting a particular decision-making relationship is rather low (mainly from one to three). This lowness is logical as the analysed sources consist of academic articles/papers in the IS field, where "IS have not been interested in publishing replications of prior studies" (Dennis \& Valacich, 2014, p. 1).

Based on the foundation offered by the hierarchy and decision-making relationships (Figure 4.8 and Figure 4.9), the business rules constraining concepts in the BPC domain has also revealed. These business rules were extracted from the knowledge sources, and represented through concepts and/or relationships of the ontology. Table 4.8 presents some examples of the business rules related to 'output quality' only. The rules define three constraints related to 'output quality': how good the crowdsourcing output is in comparison to experts'; the role of task redundancy in output quality; and the moderate role of quality control on the relationship between crowd workers and output quality. The business rules, combining with the decision-making relationships, form the reasoning knowledge guiding organisations in their BPC establishment.

Table 4.8. $\quad$ Some examples of business rules

\section{Example of business rules}

For taxonomy creation, output quality of crowdsourcing is equivalent to $80-90 \%$ of expert output (Chilton, Little, Edge, Weld, \& Landay, 2013)

The more redundancy in performing a task (in iterative workflow), the better outcome of task results (Karger, Oh, \& Shah, 2013)

Without quality control, more crowd workers are needed to achieve the same level of output quality (Tetreault, Chodorow, \& Madnani, 2014)

\subsubsection{BPC Ontology Evaluation: Triangulation}

This section discusses the evaluation of the proposed ontology. According to Venable et al. (2012), evaluation in design science helps: 1) assess artefact's utility as to whether it achieves the stated purpose; 2) compare the built artefact against other artefacts; and 3) consider side-effects and weaknesses of the artefact for future improvements. This dissertation chose to evaluate the ontology using the second approach for two reasons. First, this type of approach, compared to the other approaches, fits with the constrained 
resources of a $\mathrm{PhD}$ project. The other approaches evaluate whether the artefact works and how to improve it, which needs considerable efforts on practical, long-run applications. According to Gregor and Hevner (2013), these approaches may not be feasible in research projects with limited resources like the current case. Second, the use of different evaluation methods in a design science project has been widely suggested (Sonnenberg \& vom Brocke, 2012a; Venable et al., 2016). As the previous research stage has already used the case study evaluation and the following stage will use experiments and focus groups, the use of triangulation in the current stage can provide a complementary evaluation. As a result, triangulation, where we compared different versions of the ontology, was chosen for this evaluation.

Having adopted triangulation to evaluate the BPC ontology, we considered two metrics popular to assess ontology: clarity (Akdemir, Turaga, \& Chellappa, 2008; Fan, Hua, Storey, \& Zhao, 2016) and coverage (Fan et al., 2016; Shanks et al., 2003). We defined clarity as 'the degree to which the ontology clarifies concept meanings and reduces ambiguity in the domain', and coverage as 'the level that the ontology covers the semantics in the domain'. Using these two metrics, we compared our ontology with an ontological version generated by software.

We developed an automated version of the ontology using the same sources of information. This version was built automatically using a software tool - OntoGen that generates ontologies from text (Fortuna et al., 2007). While our ontology building was based on a detailed review of BPC sources, the automated ontology was built using the abstracts of the same sources. This is because abstracts are expected to consist of key concepts, relationships, and findings of the sources. Furthermore, the use of abstracts for ontology building is suggested by Vogrinčič and Bosnić (2011) regarding the use of article abstracts for their ontology construction. When using OntoGen to process the abstracts of BPC sources, the outcome is presented in Figure 4.10.

We then analytically compared our own ontology with the automated ontology. We find a high consistency on the main ontological elements. When comparing Figure 4.8 and Figure 4.10, a strong match is noted for the core building blocks (i.e. tasks, quality control, incentive mechanism, technical configuration, and the crowd). Furthermore, several detailed concepts are also similar, e.g. intrinsic motivation and extrinsic motivation can 
be found in both figures. Despite a few differences, we find high consistent results between the two ontological versions. As a result, the comparison validates our ontology building process through triangulation as suggested by Carlsson et al. (2011) that "to strengthen the validity of design [theories], test triangulation may be beneficial” (p. 117).

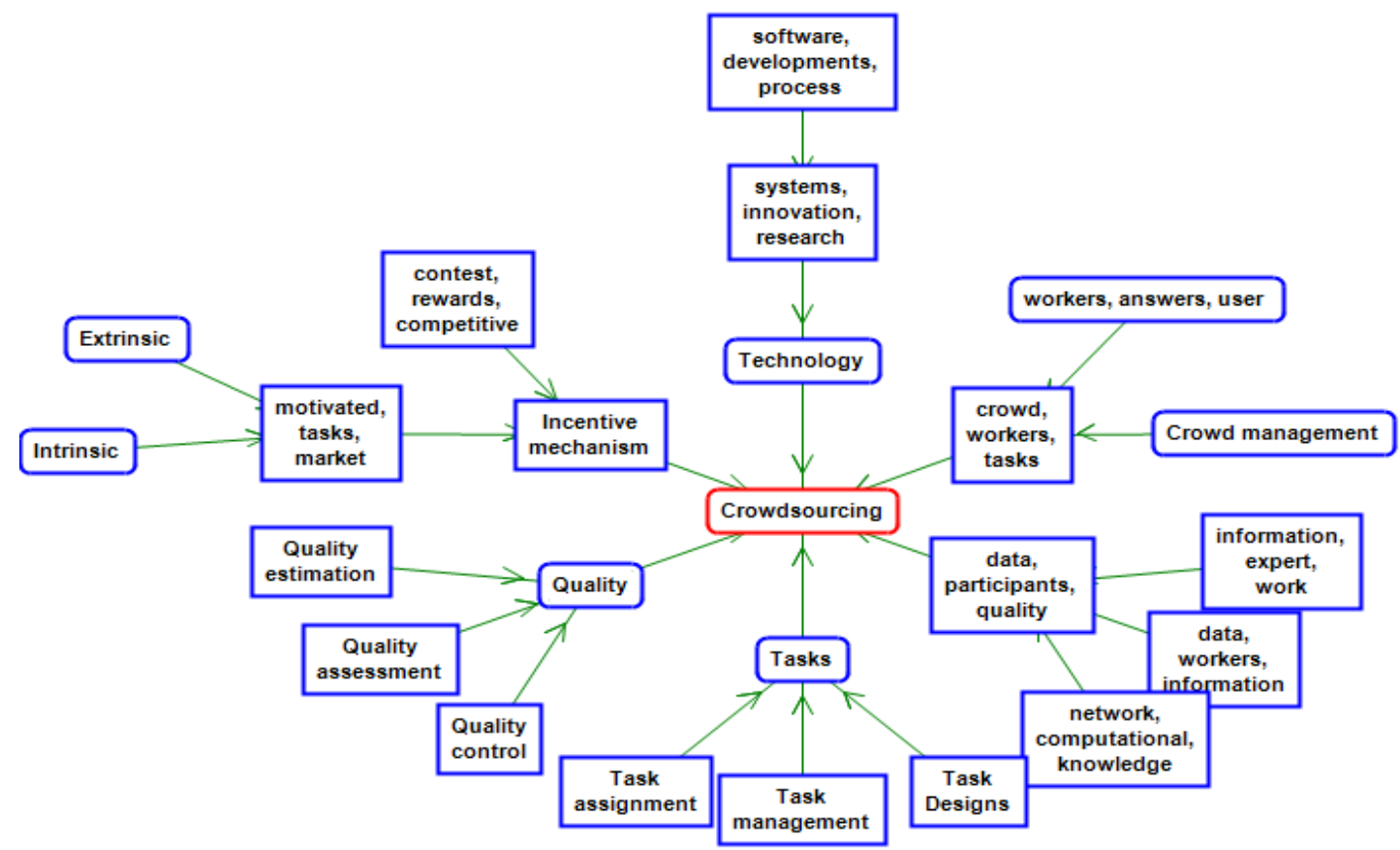

Figure 4.10. Ontological version generated by OntoGen (OntoGen was developed by Fortuna et al., 2007)

We further consider the triangulation results according to the two investigated metrics, coverage and clarity. We find that the results confirm the high coverage of the ontology. More precisely, most of the concepts generated by OutoGen have already been captured by our ontology (comparing Figure 4.8 with Figure 4.10). Further, our ontology covers not only concepts, but also hierarchical and decision-making relationships, while the automatic version is quite limited regarding the type of relationships, e.g. excluding nonhierarchical relationships. These points indicate that our ontology has covered a wider range of semantics in the domain.

Regarding clarity, our ontology advances the automatic version through two important points. First, our ontology provides clearer concept meaning. More precisely, since the automated approach groups and defines concepts based on the frequency of occurrence, rather than meaning, several extraneous composite concepts have occurred, e.g. the combination of network, computational, and knowledge. Such composite concepts do not provide clear meaning and thus make the automatic ontology harder to understand. In 
contrast, our ontology decomposes the composite building blocks into detailed concepts to make it easier to understand. Finally, our ontology distinguishes different types of concepts, e.g. building blocks, activities, data, and attributes, which is necessary for the related information system development. This capacity was not yet supported by the tool.

Given the discussion, the triangulation results allow us suggesting that the ontology highly covers and clarifies the domain semantics. Thus, we strongly believe that our approach, which builds the ontology through systematic analysis and organisation of BPC knowledge sources, is appropriate to construct the ontology of BPC. This appropriateness is supported by Osterwalder (2004) and Miah et al. (2009) regarding their similar approaches to construct domain ontologies in the IS discipline.

\subsubsection{Summary and Remarks}

This section built a lightweight and then heavyweight BPC ontology. We analysed and structured the BPC knowledge sources to identify the main concepts, hierarchical relationships, decision-making relationships, and business rules defining BPC. These elements were then organised into a lightweight ontology considering BPC building blocks, business processes, data entities, data attributes, and their hierarchy relationships. Then, decision-making relationships and business rules were added, which turned the ontology into a heavyweight ontology. To evaluate the ontology, we compared it with an automated ontology generated by OntoGen, which strengthens its validity (Carlsson et al., 2011). The results show high coverage and clarity of our constructed ontology.

The role of the BPC ontology can be seen either together with the conceptual model or as a standalone artefact. Comparing to the conceptual model, the ontology provides a more detailed decomposition of BPC, decomposing the building blocks into detailed concepts and their relationships. As a result, the conceptual model and ontology can be used as two interrelated levels of BPC decompositions, which allow organisations analyse, plan, and deploy a business process based on crowdsourcing. As a standalone artefact, the BPC ontology provides a knowledge base that consolidates the domain knowledge. It structures key concepts, hierarchical structures, and decision-making relationships of the domain, from which knowledge can be interred. With this knowledge base, instantiated artefacts can be further constructed and developed to support BPC, which is the focus of the next research stage. 


\subsection{Stage 4: Decision Tool}

This stage constructed a decision tool supporting the establishment of BPC. This construction fulfils a need in the BPC domain and plays an important role in the research process. In the BPC domain, there is a need for decision support systems to address the complexity of BPC establishment. The complexity of BPC establishment has been revealed in the previous research stages and also emphasised by other researchers (Kucherbaev et al., 2013; Tranquillini et al., 2015). More precisely, BPC establishment involves not only the several stages deploying in the crowdsourcing strategy, but also several information structures supporting the establishment (as seen via Figure 4.8). Given this complexity, it is necessary to help decision makers - managers and process designers - making analytical decisions in BPC establishment. This type of support is within the typical domain of Decision Support Systems (DSSs) (Arnott \& Pervan, 2014; Hosack, Hall, Paradice, \& Courtney, 2012). More precisely, DSSs can address the complexity by supporting managers and process designers in order to make informed decisions in BPC establishment. Consequently, the construction of a decision tool supporting BPC establishment fulfils the need in the domain.

In the research, the role of this stage is essential for both alignment with the other stages and for itself. In alignment with the other stages, this stage articulates the knowledge, which was extracted by the first stage, conceptualised by the second stage, and ontologically detailed by the third stage, in order to build a decision tool regarding the BPC process. Consequently, it contributes to move forward the theoretical efforts from the previous stages. By itself, this stage has its unique outcome and position in the research process. The outcome of this stage is a decision tool supporting BPC, which is clarified as an instantiation artefact per se (Hevner et al., 2004). Furthermore, this stage is also important due to its ultimate position in the research process. This position implies that the success of tool development has a great influence on the success of the whole project. If we can demonstrate the utility of the tool in practice, this increases confidence for not only the tool construction but also the knowledge base built into the tool.

Given the important roles, the decision tool should be constructed and evaluated in a rigorous way. In the construction, we ensured rigour through a solid knowledge base and 
an appropriate development method. Regarding the former, we built an architecture that guided the development process. This architecture embraced the BPC ontology as its knowledge base module, which solidly supported DSS development (Delir Haghighi, Burstein, Zaslavsky, \& Arbon, 2013; Miah et al., 2014). We also structured a set of decision tables based on the decision framework (Figure 4.4), which were operationalised and embraced in the tool. Regarding the development method, we followed Lim et al.'s (2008) suggestion to adopt a rapid prototyping method. This method allowed managing rigour through iterative development, assessment and revision of a few prototypes (Kordon, 2002; Lim et al., 2008). The method was appropriate for DSS development, as suggested by Miah et al. (2009) and Antunes et al. (2014).

In the tool evaluation, a combination of two different evaluation techniques enabled rigour (Sonnenberg \& vom Brocke, 2012a; Venable et al., 2016). We purposefully adopted two techniques with quite different natures: experiments and focus groups. The former is a quantitative, individual-based, and artificial evaluation, whereas the latter is a qualitative, group-based, and naturalistic evaluation. These two techniques allowed us to assess the tool not only by measuring the level of support that the tool can provide, but also by examining the participants' opinions on the usefulness of the tool. To further ensure rigour, this stage followed rigour guidance for conducting experiments suggested by Montgomery (2012), and focus groups suggested by Tremblay et al. (2010).

Based on the above discussion and in alignment between the decision tool with the conceptual model and ontology, we note that the tool should accomplish the following requirements:

- Assist managers deciding whether to establish BPC or not. This assistance should be given as guidelines and recommendations.

- Build a comprehensive, integrated view of BPC. In other words, the tool should support the integrated BPC process, not individual activities. The literature has suggested that such a comprehensive view can be reached by using sound domain ontologies (Miah et al., 2014; Osterwalder \& Pigneur, 2004).

- Support micro-decisions related to the BPC process (Figure 4.8). That is, within each building blocks, the (sub) issues, their alternatives, and guidance to choose among these alternatives should be specified.

- As an instantiation artefact, the tool should provide a means for processing and presenting knowledge related to BPC establishment. 


\subsubsection{Architecture and Decision Tables}

DSS architecture defines key components of the DSS system. Though diverse types of DSSs have been developed, their abstract architecture seems fairly consistent. Holsapple (2008) summarises this consistence and suggests an overall architecture of DSSs consisting of four components: language component, problem-processing component, knowledge component, and presentation component. Given that Holsapple's (2008) suggested components are abstract and clearly separate the important concerns of DSSs, they were adopted to design the decision tool. We note that the term 'component' was already used to refer to the model components in Section 4.2. To avoid confusion, we refer below to the architectural components as modules.

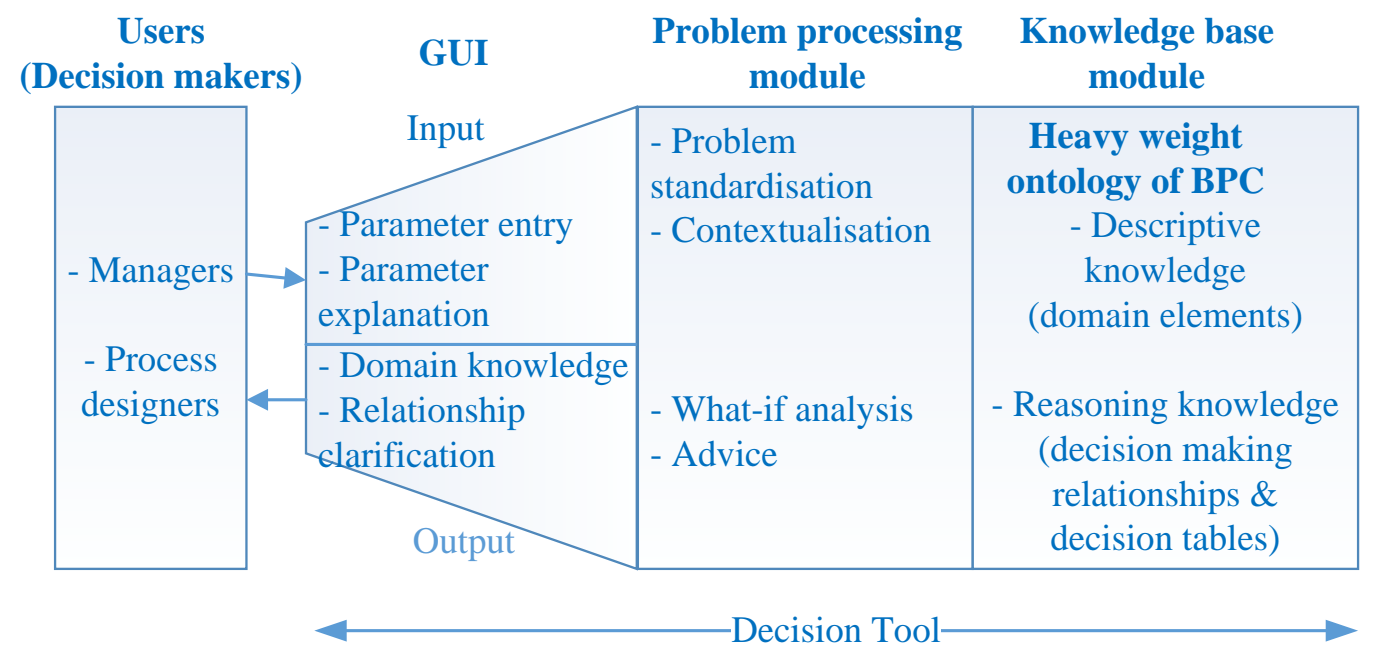

Figure 4.11. Tool architecture (adapted from Holsapple, 2008)

The tool's architecture was based on Holsapple's (2008) abstract architecture, where the language and presentation modules were combined into the graphical user interface (GUI). Consequently, the architecture consisted of three modules: GUI, problem processing module, and knowledge base module, which are depicted in Figure 4.11. The GUI module is responsible for the interaction between the tool and the users. It receives input parameters from the users, offers descriptions about the parameters, and provides related advice. The problem process module handles these inputs, where they are used to formulate the decision and the related context. This module also controls the flow of inputs by adapting what elements the GUI presents, which manipulates data entries based on the knowledge module. The knowledge module is based on the BPC ontology constructed in Section 4.3. Based on the ontology, the knowledge module can perform what-if analysis by comparing the domain knowledge with the input parameters. 
Consequently, the tool can identify inconsistencies in the inputs and provide advice on how to set up a BPC process for a particular organisational context, which in turn are presented as GUI's outputs.

In the construction of the knowledge module, we faced a challenge related to reasoning. In particular, there was a current lack of reasoning rules for making the decision to crowdsource or not (Zhao \& Zhu, 2014). Addressing the challenge, we further examined the decision factors presented in Figure 4.4 for actionable rules, which were structured by decision tables. Decision tables are suggested by Huysmans et al. (2011) as the most effective technique in terms of presentation and interpretability, compared to decision trees, propositional rules, and oblique rules. Furthermore, decision tables are easy to embed within computer software like the decision tool.

\section{Decision Tables for Making the Decision to Crowdsource}

This section presents a series of decision tables that provide actionable recommendations for making the decision to crowdsource. These tables were drawn from the decision factors (Table 4.3) and decision framework (Figure 4.4). Given the four layers of the decision framework, three decision tables were arranged according to the layers: task properties, people, and management. An exception is the environment layer that has only one decision factor, and thus does not need a separate table. Table 4.9, Table 4.10 and Table 4.11 present the three decision tables.

Table 4.9. Decision table: Task properties

\begin{tabular}{|c|c|c|c|c|c|c|c|c|c|c|c|}
\hline Conditions & & & & & & & & & & & \\
\hline Internet: Yes (Y) vs. No (N) & $\mathrm{N}$ & $\mathrm{Y}$ & $\mathrm{Y}$ & $\mathrm{Y}$ & $\mathrm{Y}$ & $\mathrm{Y}$ & $\mathrm{Y}$ & $\mathrm{Y}$ & $\mathrm{Y}$ & $\mathrm{Y}$ & $\mathrm{Y}$ \\
\hline Ease of integration with existing BP & - & $\mathrm{N}$ & $\mathrm{Y}$ & $\mathrm{Y}$ & $\mathrm{Y}$ & $\mathrm{Y}$ & $\mathrm{Y}$ & $\mathrm{Y}$ & $\mathrm{Y}$ & $\mathrm{Y}$ & $\mathrm{Y}$ \\
\hline Interactive & - & - & $\mathrm{Y}$ & $\mathrm{N}$ & $\mathrm{N}$ & $\mathrm{N}$ & $\mathrm{N}$ & $\mathrm{N}$ & $\mathrm{N}$ & $\mathrm{N}$ & $\mathrm{N}$ \\
\hline Ease of delineation & - & - & - & $\mathrm{Y}$ & $\mathrm{Y}$ & $\mathrm{Y}$ & $\mathrm{Y}$ & $\mathrm{N}$ & $\mathrm{N}$ & $\mathrm{N}$ & $\mathrm{N}$ \\
\hline Confidential information & - & - & - & $\mathrm{Y}$ & $\mathrm{Y}$ & $\mathrm{N}$ & $\mathrm{N}$ & $\mathrm{Y}$ & $\mathrm{Y}$ & $\mathrm{N}$ & $\mathrm{N}$ \\
\hline Partitionable & - & - & - & $\mathrm{Y}$ & $\mathrm{N}$ & $\mathrm{Y}$ & $\mathrm{N}$ & $\mathrm{Y}$ & $\mathrm{N}$ & $\mathrm{Y}$ & $\mathrm{N}$ \\
\hline \multicolumn{12}{|l|}{ Actions } \\
\hline Not to crowdsource & $\mathrm{X}$ & $\mathrm{X}$ & $\mathrm{X}$ & & & & & & & & \\
\hline Should crowdsource & & & & & & $\mathrm{X}$ & & & & & \\
\hline $\begin{array}{l}\text { Crowdsource with additional action } \\
\text { (CSwAA): clearly define task in the latter } \\
\text { stages of the BPC process }\end{array}$ & & & & & & & & $\mathrm{X}$ & $X$ & $X$ & $X$ \\
\hline CSwAA: hiding confidential information & & & & $\mathrm{X}$ & $\mathrm{X}$ & & & $\mathrm{X}$ & $\mathrm{X}$ & & \\
\hline CSwAA: only crowdsource as a contest & & & & & $\mathrm{X}$ & & $\mathrm{X}$ & & $\mathrm{X}$ & & $\mathrm{X}$ \\
\hline
\end{tabular}


Task properties with their central roles in the decision to crowdsource are presented in the first decision table (Table 4.9). From our knowledge base, the decision to crowdsource should only be made for tasks that satisfy three conditions: 1) can be performed through the Internet (Brabham, 2008a; Muntés-Mulero et al., 2013); 2) enable to integrate with the organisational business processes (Kittur et al., 2013; Sakamoto et al., 2011; Tranquillini et al., 2015); and 3) do not require many interactions (Afuah \& Tucci, 2012; Burger-Helmchen \& Pénin, 2010; Muntés-Mulero et al., 2013). In addition to these definite conditions, there are three other 'should-be' conditions. First, tasks should be well defined, which may be processed in the latter stages of the BPC process (Lloret et al., 2012; Muhdi et al., 2011; Zogaj et al., 2014). If tasks include confidential information, it is necessary to perform additional actions for hiding sensitive information (Feller et al., 2012; Lu et al., 2015). Finally, tasks that can be divided into small pieces of work have more chance to crowdsource (Afuah \& Tucci, 2012; Malone et al., 2010). An exception is the case of crowdsourcing contests, where several tasks that are not necessarily divisible are successfully crowdsourced in the form of open contests.

Table 4.10. Decision table: People

\begin{tabular}{|l|c|c|c|}
\hline Conditions & & & \\
\hline The crowd for task: Available (A) vs. Not available (N) & N & A & A \\
\hline Employees for task: Few (F) vs. Many (M) & - & F & M \\
\hline Actions & & & \\
\hline Not to crowdsource & $\mathrm{X}$ & & \\
\hline Should crowdsource & & $\mathrm{X}$ & \\
\hline CSwAA: consider other factors & & & $\mathrm{X}$ \\
\hline
\end{tabular}

Table 4.10 considers the roles of the availability of the crowd and organisational employees playing in the BPC process. Djelassi and Decoopman (2013) and Saxton et al. (2013) all agree on the availability of the crowd as a pre-condition for crowdsourcing. Without meeting this condition, the decision to crowdsource is inappropriate. The second condition considered in this table is the availability of internal employees. Following Afuah and Tucci (2012), we suggest that organisations should crowdsource in cases where they cannot allocated employees or neighboured agents to accomplish the tasks. In a similar vein, Lu et al. (2015) from a resource-based view advise "when a firm finds that its internal [human] resources and capabilities cannot satisfy the company's strategic objectives, the external acquisition of complementary resources and capabilities becomes 
necessary" (p. 5). Finally, if both conditions in Table 4.10 are satisfied, we suggest that crowdsourcing is still a good choice, yet advise further analysis of other factors, like task properties and management factors, before making the final decision.

Table 4.11. Decision table: Management

\begin{tabular}{|l|l|l|l|l|l|l|l|l|l|l|l|l|l|}
\hline Conditions & & & & & & & & & & & & & \\
\hline Budget: Sufficient (S) vs. Large (L) & S & S & S & S & S & L & L & L & L & L & L & L & L \\
\hline $\begin{array}{l}\text { Crowdsourcing expert: Available } \\
\text { (A) vs. Not available (N) }\end{array}$ & N & A & A & A & A & A & A & A & A & N & N & N & N \\
\hline $\begin{array}{l}\text { Acceptance level of risk: High (H) } \\
\text { vs. Low (L) }\end{array}$ & - & H & H & L & L & H & H & L & L & H & H & L & L \\
\hline $\begin{array}{l}\text { Internal commitment: High (H) vs. } \\
\text { Low (L) }\end{array}$ & - & H & L & H & L & H & L & H & L & H & L & H & L \\
\hline Actions & & & & & & & & & & & & & \\
\hline Not to crowdsource & X & & & & & & & & & & & & \\
\hline Should crowdsource & & & & & & & & & & X & X & X & X \\
\hline $\begin{array}{l}\text { CSwAA: hire outside experts (due } \\
\text { to large budget) }\end{array}$ & & & & X & X & & & X & X & & & X & X \\
\hline $\begin{array}{l}\text { CSwAA: implement mechanisms } \\
\text { for controlling risks }\end{array}$ & & & X & & X & & X & & X & & X & & X \\
\hline $\begin{array}{l}\text { CSwAA: implement strategies for } \\
\text { increasing internal commitment }\end{array}$ & & & & & X & & & & & & & \\
\hline
\end{tabular}

Table 4.11 summarises the decision factors regarding management aspects: budget, availability of crowdsourcing experts, risk, and internal commitment. Several studies suggest that a sufficient budget is required for a crowdsourcing decision (Djelassi \& Decoopman, 2013; Lofi, Selke, \& Balke, 2012; Lu et al., 2015). Though the cost of crowdsourcing activities is usually small, other related costs such as quality control, service costs, coordination costs, and incentive mechanisms, may be significant. As a result, crowdsourcing should be appropriate for projects where the budget is sufficient. That is, the budget is not enough to perform the tasks in a traditional way, i.e. internal sourcing or outsourcing (Malone et al., 2010), but enough to cover the BPC process (Lu et al., 2015). In addition, crowdsourcing expertise and experience is necessary to coordinate the activities (Muhdi et al., 2011; Rouse, 2010). Thus, if a project has limited crowdsourcing expertise, hiring outside experts should be considered. Where hiring cannot be arranged due to limited budget, the project should not be crowdsourced.

Crowdsourcing also needs the project to have a high level of risk acceptance and internal commitment. As crowdsourcing relies on anonymous members of the crowd, it involves 
several risks, including low quality results and loss of intellectual property (Kannangara \& Uguccioni, 2013; Naroditskiy et al., 2013; Schenk \& Guittard, 2011). Consequently, like any other sourcing projects, mechanisms for controlling risks should be implemented. Furthermore, another factor that can jeopardise the adoption of crowdsourcing is to have a low level of employees' commitment to crowdsourcing (Brabham, 2008a; Lüttgens et al., 2014; Simula, 2013). This is because internal employees may fear losing their jobs because of crowdsourcing and thus create barriers for its adoption. To increase internal commitment, we suggest empowering key individuals who drive the crowdsourcing project (Lüttgens et al., 2014), and restructuring the internal incentive systems similar to what has been done in open innovation in order to overcome employees' negative attitudes (Huston \& Sakkab, 2006).

Finally, as the lone environmental factor of the decision framework (Figure 4.4), the availability of crowdsourcing platforms should be evaluated. Several researchers suggest the high availability of platforms is often critical for crowdsourcing activities (Chanal \& Caron-Fasan, 2010; Lüttgens et al., 2014; Zogaj et al., 2014), though it is also possible to build an organisational crowdsourcing platform. The reasons for adopting existing platforms include the large pool of crowd members (Mason \& Suri, 2012), low setup efforts (Wang, Hoang, \& Kan, 2013) and, in some cases, protection of intellectual property (Feller et al., 2012). Agreeing with these benefits, we note that there are two types of existing platforms: specialised and horizontal platforms. Specialised platforms concentrate on particular types of tasks, e.g. InnoCentive for problem solving tasks (Hirth et al., 2011), and thus have their own specialised members. Horizontal platforms, like AMT, may address different types of tasks and thus have diverse crowd members (Kucherbaev et al., 2013). This distinction may also influence the choice of using existing platforms. For instance, a crowdsourcing project having multiple dissimilar tasks may be more suited to a horizontal platform than a specialised one.

In summary, these decision tables have captured the reasoning rules for making the decision to crowdsource. With these reasoning rules, we are now ready to develop the decision tool. 


\subsubsection{Tool Development}

Looking back to Figure 4.11, the tool architecture was used to guide our development. As this development followed the rapid prototyping method (Lim et al., 2008), it was realised through two prototypes. Both prototypes were designed as web applications, using PHP and MySQL. The first prototype was developed and assessed in order to provide revision feedback. The second prototype development used this feedback to improve its functionality, and served as a tool supporting decision makers making informed decisions in BPC establishment. The following sections describe each of them.

\subsubsection{The First Prototype}

We developed a decision prototype to support the establishment of BPC. Concerning two main types of decision-makers in the BPC process, namely project managers and process designers, the prototype was designed with two main functions: Tool 1 and Tool 2. Tool 1 defines the project context, and analyses whether to crowdsource or not using the decision tables (Table 4.9, Table 4.10, and Table 4.11). As a result, Tool 1 may suggest whether crowdsourcing is an appropriate choice for the project or not, and possible actions that may increase (or decrease) the probability of crowdsourcing. Tool 2 suggests the main workflows in a BPC process, i.e. task design, workflow design, crowd management, incentive mechanism, quality control, technical configuration and output (aligning with the innermost layer of the ontology - Figure 4.8). Within each workflow, the tool suggests activities that should be operationalised and design options that support the operationalization. The outputs of Tool 2 are the concrete designs and related what-if advice. The GUI of the two tools are presented in Figure 4.12 and Figure 4.13. 


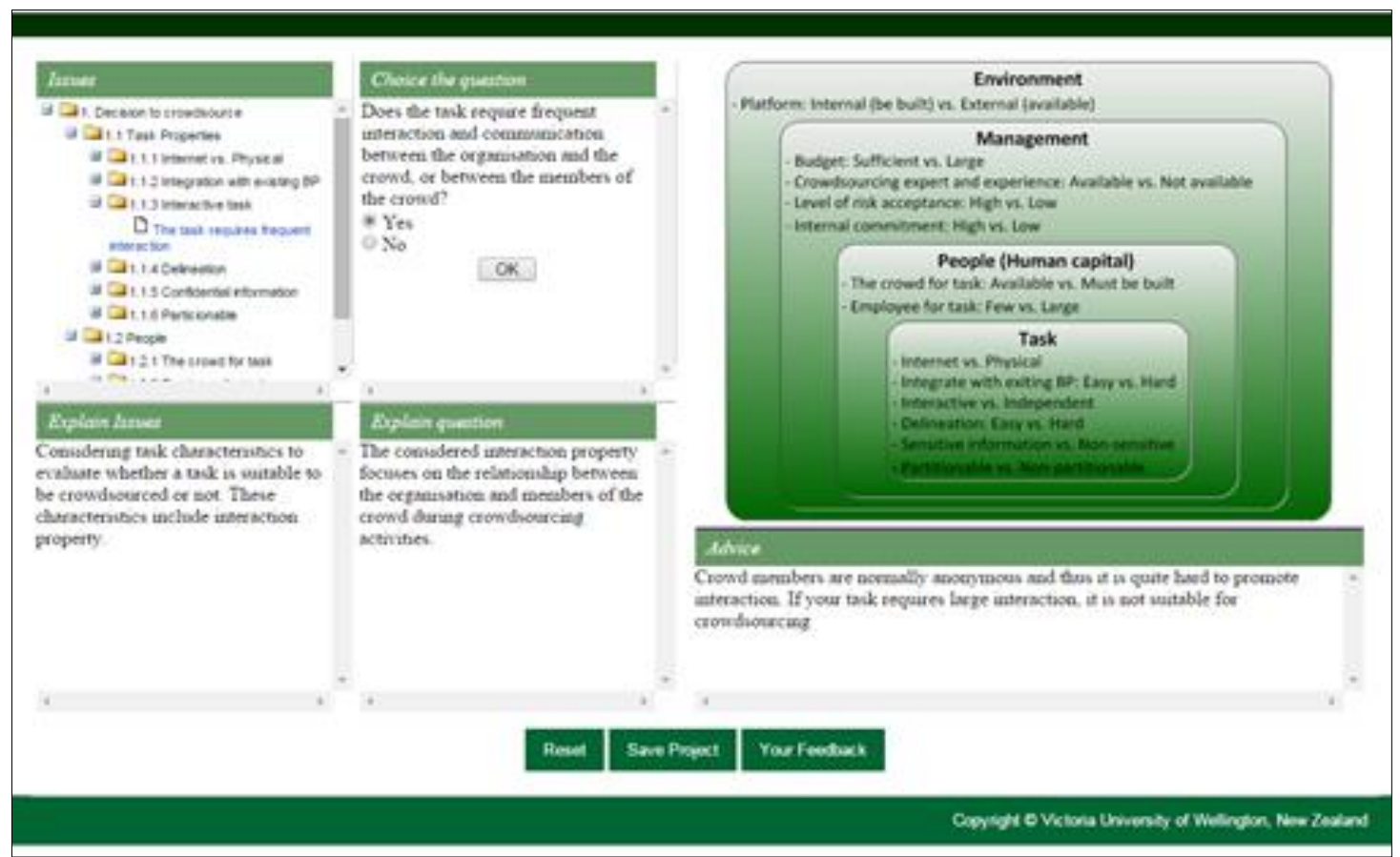

Figure 4.12. Tool 1: The decision to crowdsource

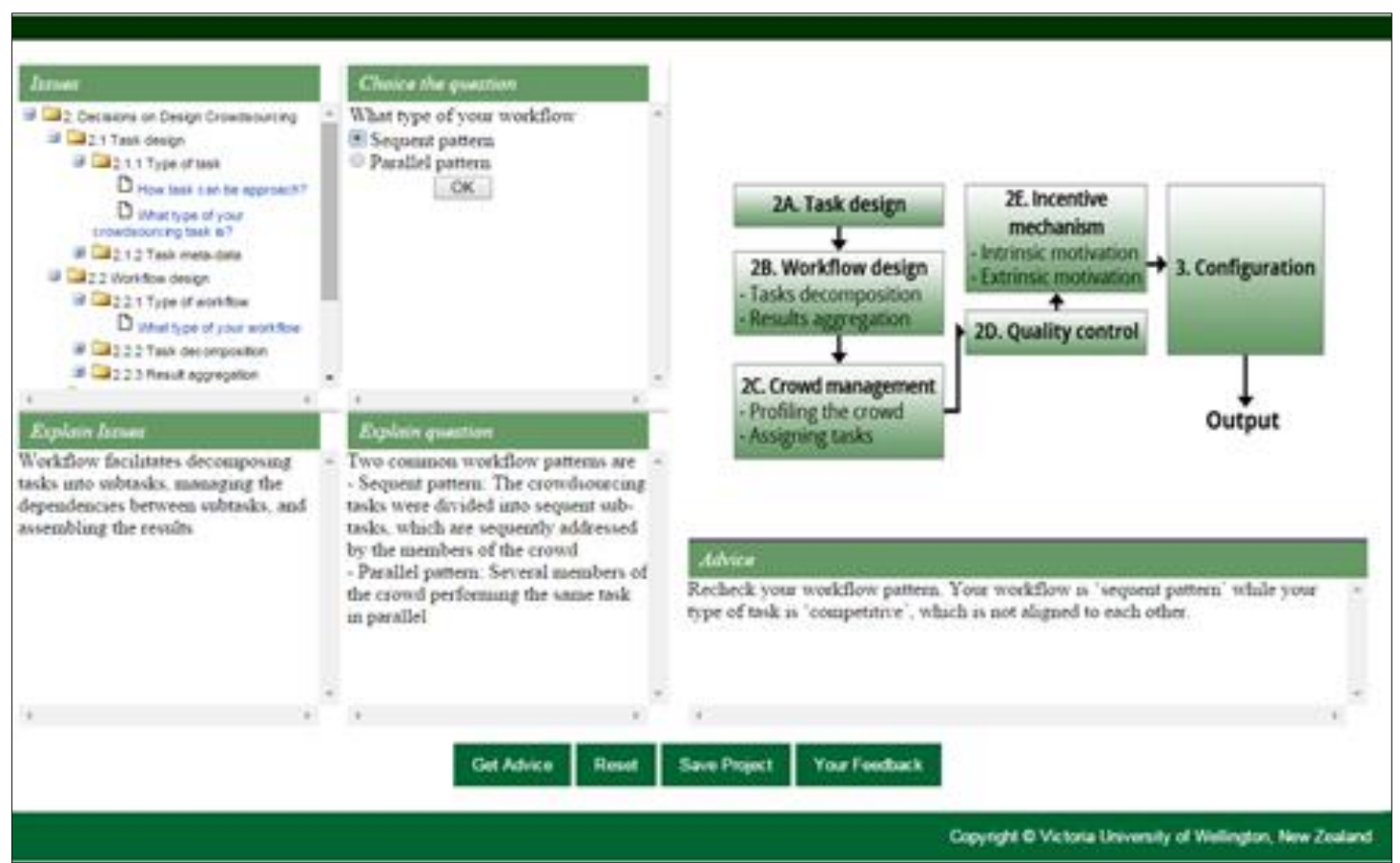

Figure 4.13. Tool 2: Process design of BPC establishment

Even though the two tools served different purposes, they were intentionally designed with a consistent user-interface. The GUIs were organised in four areas, abstractly presented in Figure 4.14. The left-hand side was dedicated to user inputs, allowing users to navigate within pre-defined issues (decision issues and design issues). These issues 
were aligned with the overview diagrams of the decision framework and design process, presented in the right-hand side. The middle area presented a pre-defined question and input parameters according to the chosen issue. When the user answers the question, the tool provided appropriate advice based on the reasoning knowledge.

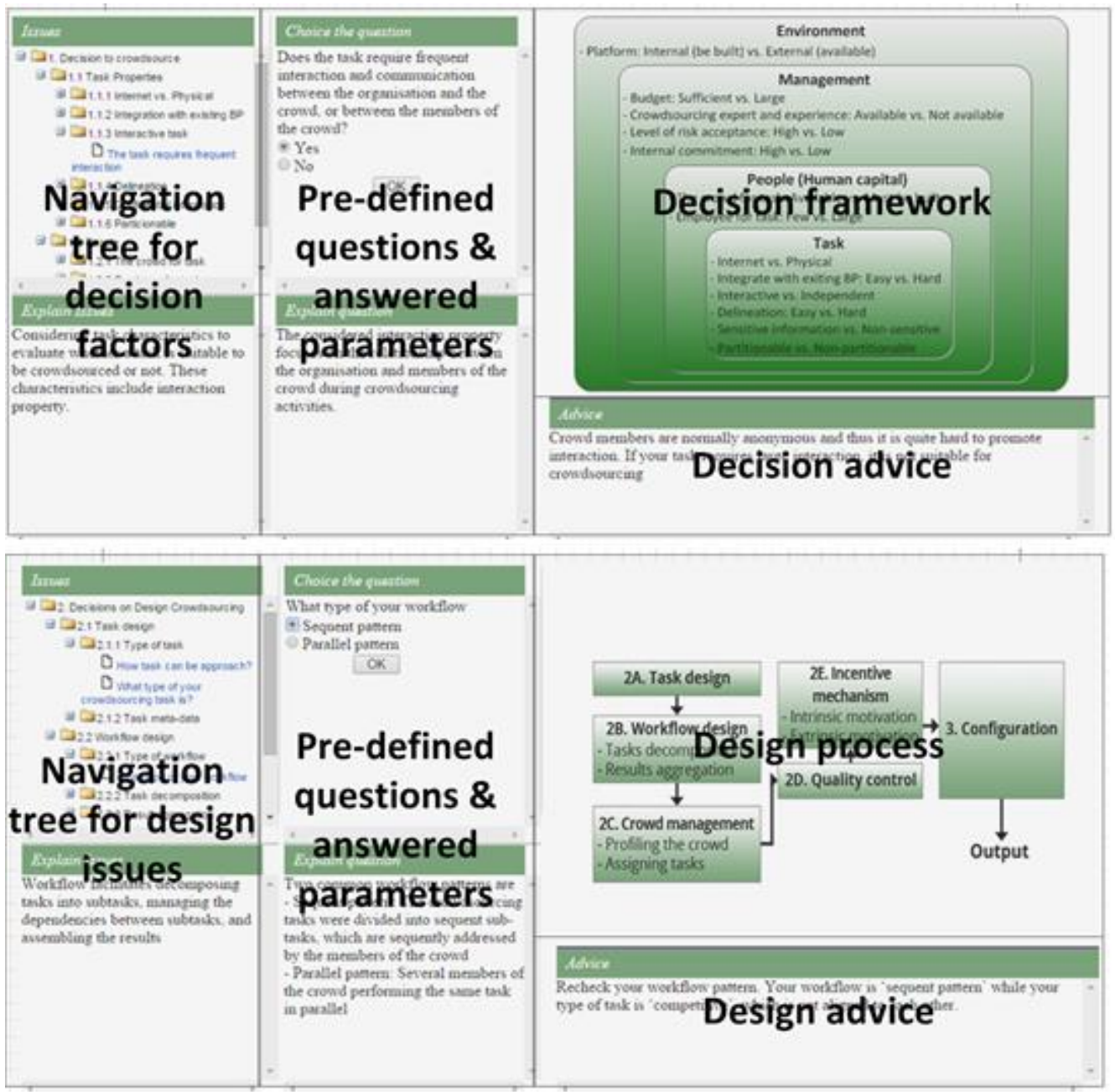

Figure 4.14. Abstract views of Tool 1 and Tool 2

This prototype had to be evaluated to provide feedback for the next round of development. To evaluate the prototype, a pilot experiment was conducted. The aim of the experiment was twofold. The first aim came from the prototyping perspective that determined whether the tool met its performance requirements and thus helped to identify possible improvements. The second aim, which originated from the evaluation point of view, sought to test the experimental materials for the evaluation of the tool (Dennis \& Valacich, 2001). In the subsequent section, we focus on the first aim, while the second one will be discussed in the later sections. 


\subsubsection{Evaluation: Pilot Experiment}

The prototype was evaluated through a pilot experiment. In the experiment, we grouped participants into two groups: A and B. We asked all participants to perform two exercises, which were designed according to the decision to crowdsource and the process design. In exercise 1 (testing Tool 1), group A was the control group performing the exercise without the tool, while group B was the treatment group using the tool. In exercise 2 (testing Tool 2), the roles of the two groups were swapped to test Tool 1 and Tool 2 independently. The performance of participants was measured through their answers on the two exercises. In this experimental design, the prototype would be considered useful if the treatment group's performance outperforms the control group's. Besides analysing performance, we also observed how the participants used the tool during the experiment. This observation, combined with the participants' review, provided feedback to revise the prototype.

Overall, 49 students participated in the experiment. They were second and third-year IT students in the Can Tho University of Technology (CTUET). During the experiment, three of them did not use the prototype as requested, and thus their answers were removed from the final dataset. As a result, there were 46 participants remaining: 26 participants in group A and 20 participants in group B. Table 4.12 presents the final sample, and Figure 4.15 shows pictures of the two groups in the experiment.

Table 4.12. Design of pilot experiment

\begin{tabular}{|l|l|l|}
\hline & $\begin{array}{l}\text { Exercise 1: decision to } \\
\text { crowdsource }\end{array}$ & $\begin{array}{l}\text { Exercise 2: crowdsourcing } \\
\text { process design }\end{array}$ \\
\hline $\begin{array}{l}\text { Group A } \\
\text { (26 participants) }\end{array}$ & $\begin{array}{l}\text { Control group } \\
\text { (without Tool 1) }\end{array}$ & $\begin{array}{l}\text { Treatment group } \\
\text { (using Tool 2) }\end{array}$ \\
\hline $\begin{array}{l}\text { Group B } \\
\text { (20 participants) }\end{array}$ & $\begin{array}{l}\text { Treatment group } \\
\text { (using Tool 1) }\end{array}$ & $\begin{array}{l}\text { Control group } \\
\text { (without Tool 2) }\end{array}$ \\
\hline
\end{tabular}
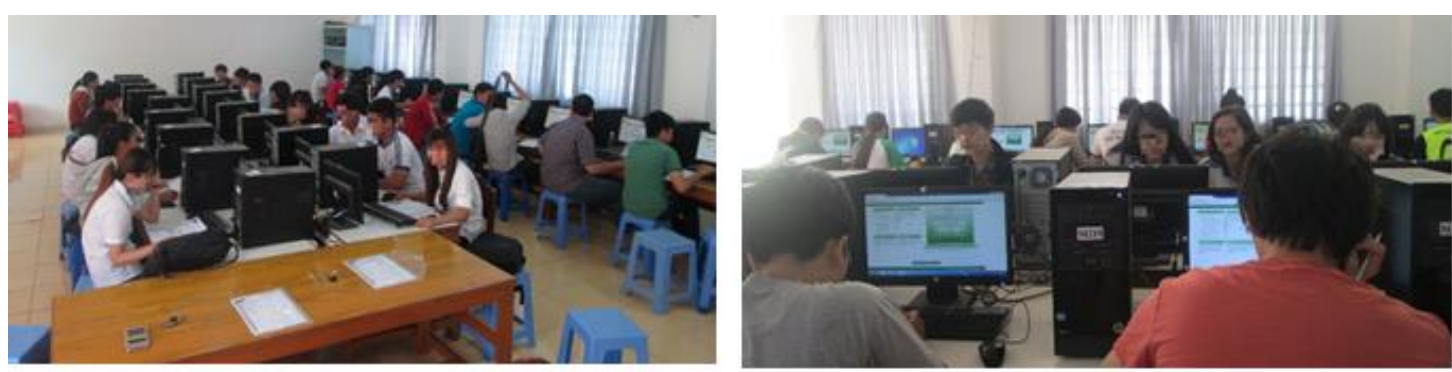

Figure 4.15. Pilot experiment: Pictures of Group A (left) and Group B (right) 


\section{Pilot Experiment Results}

We received 46 valid answers from the participants. Based on pre-defined standard answers, we calculated solution scores for each answer. More precisely, we used the following formulation: A correct answer was scored 1; 'No Idea' was scored 0.5; a wrong answer was scored 0 . With the formulation, the participants' score on each exercise was calculated. Given that each exercise consisted of four questions, the score scale ranged from 0 to 4.

Table 4.13. Pilot experiment: Score frequency in the two exercises

\begin{tabular}{|r|r|r|r|r|}
\hline Score & \multicolumn{2}{|c|}{ Exercise 1 } & \multicolumn{2}{c|}{$\begin{array}{c}\text { Exercise 2 } \\
\text { (Swapped the group role) }\end{array}$} \\
\cline { 2 - 5 } & $\begin{array}{c}\text { Group A - } \\
\text { without the tool }\end{array}$ & $\begin{array}{c}\text { Group B - } \\
\text { using the tool }\end{array}$ & $\begin{array}{c}\text { Group A - } \\
\text { using the tool }\end{array}$ & $\begin{array}{c}\text { Group B - } \\
\text { without the tool }\end{array}$ \\
\hline 2 & 2 & 8 & 3 & 3 \\
\hline 2.5 & 3 & 2 & 0 & 2 \\
\hline 3 & 18 & 10 & 12 & 7 \\
\hline 3.5 & 0 & 0 & 0 & 1 \\
\hline 4 & 3 & 0 & 11 & 7 \\
\hline
\end{tabular}

Table 4.13 presents the frequency of scores in the experiment. Via Table 4.13, it seems that in exercise 1 the group without the tool outperforms the other group, while in exercise 2 it is the group using the tool has higher performance. This can partly be seen via the highest scores (the last row in Table 4.13) of each group in both exercises. To further confirm this observation, we statistically analysed the data. As the data are not normally distributed, as suggested by the Shapiro-Wilk tests, we analysed the data using MannWhitney tests (Pfeiffer, Benbasat, \& Rothlauf, 2014). The descriptive data and the pvalues of the Mann-Whitney tests are presented in Table 4.14 and Table 4.15.

Table 4.14. Descriptive data and p-value: Exercise 1

\begin{tabular}{|l|c|c|c|c|c|c|c|}
\hline \multirow{2}{*}{ Exercise 1 } & \multirow{2}{*}{ p-value } & \multicolumn{3}{c|}{$\begin{array}{c}\text { Without Tool 1 } \\
\text { (Group A) }\end{array}$} & \multicolumn{3}{c|}{$\begin{array}{c}\text { Using Tool 1 } \\
\text { (Group B) }\end{array}$} \\
\cline { 3 - 9 } & & $\boldsymbol{N}$ & Mean & Std. & $\boldsymbol{N}$ & Mean & Std. \\
\hline Solution score & 0.08 & 26 & 2.98 & .48 & 20 & 2.55 & .48 \\
\hline
\end{tabular}

Table 4.15. Descriptive data and p-value: Exercise 2

\begin{tabular}{|c|c|c|c|c|c|c|c|}
\hline Exercise 2 & p-value & \multicolumn{3}{|c|}{$\begin{array}{c}\text { Without Tool 2 } \\
\text { (Group B) }\end{array}$} & \multicolumn{3}{c|}{$\begin{array}{c}\text { Using Tool 2 } \\
\text { (Group A) }\end{array}$} \\
\cline { 3 - 8 } & & $\boldsymbol{N}$ & Mean & Std. & $\boldsymbol{N}$ & Mean & Std. \\
\hline Solution score & .51 & 20 & 3.18 & .73 & 26 & 3.31 & .68 \\
\hline
\end{tabular}


Table 4.14 and Table 4.15 show mixed results regarding the usefulness of the tools. More precisely, the group using Tool 1 has a lower score compared to the other group (Table 4.14), while the group using Tool 2 has a higher score compared to the other group (Table 4.15). However, the differences between the groups with and without the tool in both exercises are not significantly supported at the significant level of 0.05 ( $p$-value $=0.08$ for exercise 1 and $p$-value $=0.51$ for exercise 2 ). Given these results, we cannot reject the assumption that there are differences between the treatment and control groups.

Although the differences are not significantly supported, they indicate two important points. Regarding Table 4.15, the slightly higher performance of participants using the tool (mean of 3.31 versus 3.18) is likely enough to warrant further study using Tool 2 for supporting the crowdsourcing process design. From the results, we expect that in further evaluation the tool will show its usefulness in designing the crowdsourcing processes. Regarding Table 4.14, participants using the tool are less performance than the others (mean of 2.55 versus 2.98 ). This suggests that this early prototype may not fully satisfy the design requirements. Thus, it is necessary to analyse participants' feedback for revision and development of the second prototype.

\subsubsection{Feedback for Revision and the Second Prototype}

Given the mixed results of the first prototype performance, this step assessed and identified feedback to further improve the tool. The use of the feedback loop from the evaluation to improve the succeeding design has been suggested by several design science researchers (Mettler, Eurich, \& Winter, 2014; Sonnenberg \& vom Brocke, 2012a). For this purpose, we analysed the participants' comments and our observation of how the participants interacted with the tool during the experiments. The analysis led to three important notes for the tool revision.

First, the tree structure of the prototype offered an effective way to access decision/design issues. We observed that participants preferred to use this structure to navigate to the issues that were most related to their context rather than step-by-step accessing them, which was aligned with what managers and process designers actually do in practice. As a side effect of too fast navigation, we observed that some participants jumped into the final decision (and answered questions in the exercises) based only on addressing one 
certain issue, rather than considering all related issues. This might lead to incorrect answers due to incomplete context awareness, which was particularly true for exercise 1, where the decision to crowdsource required considering all related factors. This might partly explain the unexpected results of using Tool 1. Given the discussion, one necessary revision was to keep the tree structure for navigation and to add a function for the final project's advice, complementary to the issues' advice.

Second, backing the tool with the BPC ontology was appropriate. During the experiment, the participants were effectively supported by having concept explanations, alternative parameters, and advice. This effectiveness could be clearly seen with Tool 2, which increased the performance mean in Table 4.15. Such support was enabled in the tool through the ontology. The appropriateness of using ontologies for founding the DSS design is suggested by Miah et al. (2014) and Amailef and Lu (2013) regarding the use of ontologies supporting DSSs in rural business operators and emergency response respectively.

Finally, besides our own observation, we also asked participants at the end of the experiment to give feedback for improving the tool. We received some major feedback related to loading time and working space. Regarding the loading time, some participants complained about the long waiting time. As the first prototype did not use interactive programming languages, it loaded a few times for presenting questions, parameters, question definition and advice. The loading time became longer when the number of participants accessing the tool increased. Addressing this issue, the next prototype should provide the information instantly, which required using interactive programming languages like JavaScript. Regarding working space, some participants suggested removing the model and process diagrams in the tools in order to provide more working space, i.e. issues, questions, and advice. Basically, the users preferred faster interaction and a simpler interface where the issue and advice functions are the focus.

\section{The Second Prototype}

Presenting in Figure 4.16 and Figure 4.17, the second prototyping addressed the three aforementioned notes. First, it continued using the tree structure for navigation, and further added the project's advice (area 1 in the figures). Consequently, users can receive both advice for a particular aspect and integrated advice for the whole project. Second, 
we kept using the BPC ontology for backing this prototype. Furthermore, the role of the ontology was extended to serve as a basic profile of crowdsourcing projects. This profile can be adapted regarding project conditions and intervention plans. Through this adaption, the tool can detect any inconsistencies in the input data, and provide advice for the whole project.

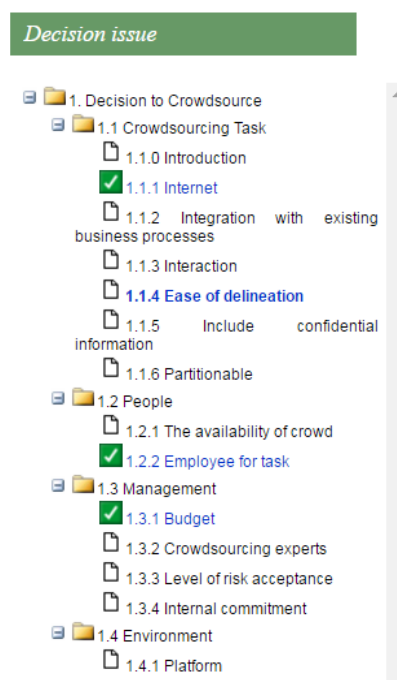

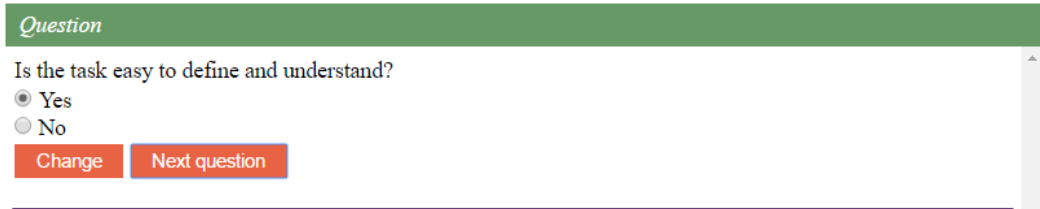

1.1.4 Ease of delineation

This property refers to how the task is defined. Normally, requirements and expected outcomes need to be specified and to be easy for the crowd to understand.

\section{Advice}

This property in your project (easy of task delineation) is suitable with crowdsourcing. In addition, more information is needed to assess the whole project for the final decision.

- Use the decision tree on the left-hand side to navigate to the decision issue.

- Once all the project information was input, you may choose: Summary Project \& Advice to access the report for the whole project OR Save Project to save the project information and return to the list of projects.

\section{Project Summary \& Project Advice $\quad$ Save Project}

Figure 4.16. Second prototyping: Decision to crowdsource (Tool 1)

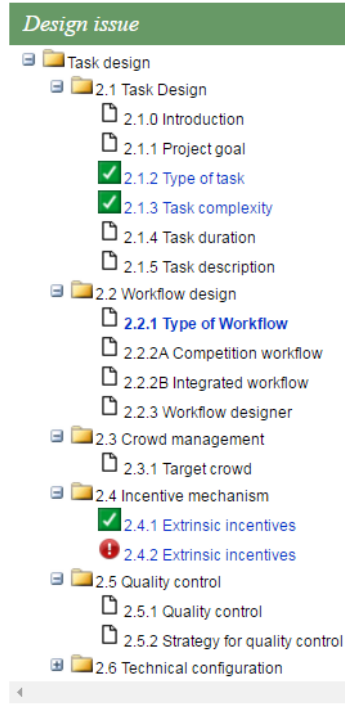

\section{Question}

What does the workflow of the project looks like?

- Competition workflow

Divide-and-integration workflow

Change Next question

\subsubsection{Type of Workflow}

2 popular workflow types are:

- Competition workflow: the crowd members will perform the same task, and the best results will be chosen - Divide-and-integration: Tasks will be divied into sub-tasks, and their results will be integrated at the end

\section{Advice}

Navigate to question 2.2.2A for choosing suitable steps of the competition workflow.

- Use the decision tree on the left-hand side to navigate to the decision issue.

- Once all the project information was input, you may choose: Summary Project \& Advice to access the report for the whole project OR Save Project to save the project information and return to the list of projects.

Project Summary \& Project Advice

Save Project

Figure 4.17. Second prototype: Process design (Tool 2) 
Finally, the user interface of the second prototype was also updated. The updated interface consisted of three main areas: issue, input, and advice. The decision issue was located in the left-hand side, which presents the pre-defined issues and provides an overview about the decision making process. As already mentioned, it allowed users to navigate by a tree structure to choose an issue. The input area was presented in the (top) right-hand side. It presented a pre-defined question and parameters according to the chosen issue, as well as an explanation of the question and parameters. The bottom right-hand side was dedicated to providing advice. We noted that advice here is based on the contingent information specified by the users. Consequently, they could change when more information was provided. As a result, the complete advice was provided after the user declared all the related information. Besides, JavaScript as an interactive programming language was also added to the interface in order to provide instant interaction.

For further interaction with the prototype, we invite the interested readers to visit https://crowdsourcing.sim.vuw.ac.nz (username: SIM20; password: SIM20). In what follows, we detail the empirical evaluation of the second prototype for its utility and perceived usefulness using experiments and focus groups.

\subsubsection{Evaluation: Experiment}

\subsubsection{Experimental Overview}

To collect empirical evidence about the utility of the decision tool, we conducted a series of six experiments. The use of experiments as an evaluation method has been suggested by both design science literature (Hevner \& Chatterjee, 2010; Peffers et al., 2012; Venable et al., 2016), and DSS literature (Rhee \& Rao, 2008). The suggestion is clarified by Mettler et al. (2014) that "design experiments are an ideal technique for testing newly developed artifacts and for systematically deriving design improvements" (p. 224). In the current study, the experiments can be further characterised as ex-post and artificial evaluation. According to Venable (2012), the ex-post characteristic refers to the evaluation of an instantiated artefact, which is the decision tool in our research. The artificial characteristic is related to the controlled nature of the experiments, which assesses the tool in a lab setting. 
We designed and conducted the experiments following the standard procedures for experimental research suggested by Montgomery (2012). The aim of the experiments was to study the utility of the tool in supporting the BPC decisions. For this purpose, we defined utility as 'having the ability to make difference on the performance between participants using the tool and others who do not use the tool'. Naturally, the experiments followed a simple comparative model with two conditions: using and not using the tool (Montgomery, 2012). This defined the independent variable of the experiments as the level of decision support: the usage and absence of the tool. Given the two supporting levels, groups of participants that used the tool were treatment groups, while the others were control groups. The dependent variable was the decision-making performance. All participants were asked to perform two exercises, designed according to the decision to crowdsource and the process design. The performance was measured through the participants' answers on the two exercises. Intuitively, the tool would be considered useful if the treatment groups had a higher performance compared to the control groups.

Given the discussion and the tool consisting of two distinct functions, supporting the decision to crowdsource (Tool 1) and crowdsourcing process design (Tool 2), two hypothesises were proposed:

Hypothesis 1 (H1): The usage of Tool 1 leads to better performance on making the decision to crowdsource.

Hypothesis 2 (H2): The usage of Tool 2 leads to better performance on designing crowdsourcing processes.

We expected that both hypotheses could hold. That is, the decision tool could increase user performance in both activities of BPC and thus demonstrate its utility.

\subsubsection{Experimental Design}

As comparative experiments, we could simply design the experiments with the treatment groups using both Tool 1 and Tool 2, and the control groups without either of them. However, such a design may generate a learning effect. That is, the participants, when they come to Tool 2, already have experience with using Tool 1, which may prevent our ability to evaluate the two tools separately. Given that, we decided to swap the group roles in the two exercises, i.e. group A was the control group in exercise 1 and then was changed 
to the treatment group in exercise 2; the swapping role of group B was vice versa. Table 4.16 represents this role swapping.

Table 4.16. Experimental design: Swapping roles between the two groups

\begin{tabular}{|l|l|l|}
\hline & Exercise 1: decision to crowdsource & \multicolumn{1}{c|}{ Exercise 2: process design } \\
\hline Group A & Control group (without Tool 1) & Treatment group (using Tool 2) \\
\hline Group B & Treatment group (using Tool 1) & Control group (without Tool 2) \\
\hline
\end{tabular}

\section{Participants}

Participants were recruited from students of the Can Tho University of Technology (CTUT), Vietnam. The participants received a 10 NZD gift card for mobile phone recharge. To keep the participants homogeneous, the recruitment was based on class units, which were a combination of major and study year in CTUT. In particular, we recruited second and third year students, with a background in Information Technology (IT) and Industrial Management (IM). As a result, six experimental sessions were formed. In each session, the participants were randomly assigned to group A and group B. The numbers of participants in each session are presented in Table 4.17.

Table 4.17. Numbers of participants per session (chronological order)

\begin{tabular}{|c|c|c|r|c|c|}
\hline Session & Major & $\begin{array}{c}\text { Study } \\
\text { Year }\end{array}$ & $\begin{array}{c}\text { Number of } \\
\text { participants }\end{array}$ & $\begin{array}{c}\text { Group A } \\
\text { (Exercise 1 without } \\
\text { the tool; exercise 2 } \\
\text { using the tool) }\end{array}$ & $\begin{array}{c}\text { Group B } \\
\text { (Exercise 1 using } \\
\text { the tool; exercise 2 } \\
\text { without the tool) }\end{array}$ \\
\hline $\mathbf{1}$ & IM & Second & 19 & 10 & 9 \\
\hline $\mathbf{2}$ & IT & Second & 40 & 22 & 19 \\
\hline $\mathbf{3}$ & IM & Third & 38 & 18 & 20 \\
\hline $\mathbf{4}$ & IT & Third & 25 & 12 & 13 \\
\hline $\mathbf{5}$ & IT & Second & 24 & 15 & 9 \\
\hline $\mathbf{6}$ & IM & Second & 44 & 21 & 22 \\
\hline
\end{tabular}

We acknowledged the concerns of using students as proxy for crowdsourcing practitioners, in particular, with respect to their crowdsourcing experience. However, the tool was designed in a way that general practitioners, with less experience, can benefit from using the tool. Thus, students with less experience are appropriate for testing the tool. Furthermore, the use of students as experimental participants is very popular in software engineering (Sjøberg et al., 2005), and is considered appropriate to test computer-based tools (Dennis \& Valacich, 2001). 


\section{Procedure and Materials}

All sessions of the experiments were conducted between July and September 2015. Starting each session, participants were tutored to become familiar with the crowdsourcing concept and instructed on how to use the tools. They were then randomly placed into two computer labs according to groups A and B. Participants then had to complete exercise 1 and then exercise 2, each of which took about 30 minutes. The same exercises were delivered to both group A and group B. The only difference between the two groups was the treatment, where one group was instructed to use Tool 1/Tool 2 for addressing the exercises while the other group was not. At the end of each exercise, the participants handed their answers to the researcher.

The two exercises were developed in relation to the BPC decisions. In particular, exercise 1 , focusing on the decision to crowdsource, included four different scenarios where crowdsourcing was a possibility. Each scenario had a short description and a question asking 'should the task [in the scenario] be crowdsourced?'. To make the scenarios diverse and close to practice, the scenario descriptions highlighted different decision factors, which were adapted from Afuah and Tucci (2012). Exercise 2 addressed issues related to the design of BPC processes. It consisted of two scenarios based on the case studies described in Section 4.2.2. Each scenario had a description and two design questions asking about different aspects of the BPC processes: task division, task description, incentive mechanism, and quality control. These aspects were adopted from Kittur et al. (2013). An experimental sheet is presented in Appendix F.

Regarding the questions of the exercises, each of the two exercises consisted of four questions (yes/no and multiple-choice). We also asked the participants to explain their reason(s) for making the choice. The questions asked several crowdsourcing aspects, which were based on other studies to increase the neutrality of the experimental evaluation (Afuah \& Tucci, 2012; Kittur et al., 2013). Also for the neutral purpose, when answering the questions, the participants might choose 'No Idea' if they thought that the scenarios did not provide enough information to answer a particular question.

By the end of experimental sessions, the participants were asked to complete a short survey on the perceived usefulness of the tool. The survey consisted of four questions adopted from Venkatesh and Davis (2000). 


\section{Pilot Experiment}

A pilot experiment was conducted, serving two purposes. First, from a tool design point of view, the pilot experiment aimed at evaluating the tool for improving its design. Second, from an experimental point of view, the pilot experiment served as a test to refine the experiment materials (Dennis \& Valacich, 2001). Since the first purpose and the results of the pilot experiment were already presented in Section 4.4.2.2, the second purpose is presented here.

Learning from the pilot experiment, a few changes had been applied to the main experiments. First, the pilot experiment recruited only students with the IT background, which might lead to limited results in the decision to crowdsource since IT students might neglect a managerial focus. The main experiments extended the recruitment to include both IT and management students. The mix of students with different backgrounds also contributed to the robustness of the main experiment. Second, the lowest score in both exercises in the pilot experiments was two, which was quite high in a 0-to-4 range. This indicated that the pilot exercises were not complex enough to discriminate results (Dennis \& Valacich, 2001). Addressing this issue, the main experiments increased the complexity of the exercises and asked participants to justify reasons for their answers. Finally, we already noted in the pilot experiment that some participants did not use the tool and answered the questions randomly. To increase the possible interaction, the main experiments asked participants to save their interaction with the tool.

\section{Measurement}

Solution scores were used to measure the participant performance. Due to the increased complexity of the exercises, scoring the participants' answers was not a straightforward task. Given that, a marking team was formed with four lecturers from CTUT (excluding the researcher). This team started by making standard answers and formulating the score. They formulated the score as: a wrong answer was scored 0; a correct answer was 0.5 , plus meaningful explanation was an additional 0.5 ; 'No idea' was either 0 or 0.5 depending on the explanation. This meant each answer scored either 0 (zero), 0.5, or 1. Given the four questions and answers in each exercise, the score scale was from 0 to 4.

Using this formulation, the team started by marking together ten participants' answers and discussed any differences. After discussion and consensus on the marking, they did 
their marking individually. Additionally, the marking was arranged in a way that each session, i.e. both control and treatment groups, was marked by a single marker, which could reduce marking bias when comparing the score between the two groups. At the end of the marking process, the researcher compared the means of the scores among the sessions. One session had quite a high mean compared to others. A moderation meeting was organised to review the marks of the session, leading to a few changes on its scores.

\subsubsection{Experimental Results}

Overall, 190 students participated in the experiments. All participants' answers were scored and recorded in the sample. Starting the analysis, we tested the normality assumption of the sample. As the solution scores were treated as discontinuous measures (the minimum difference between two scores is 0.5 ), we expected that the normality assumption might not hold, which was confirmed by the Shapiro-Wilk tests, i.e. $p$-values $<0.001$ for both exercises. These results strongly guided our choice of statistical tests in the following analysis. Table 4.18 shows descriptive statistics of the sample regarding each session.

Table 4.18. Descriptive results of six experimental sessions

\begin{tabular}{|c|c|c|c|c|c|}
\hline Exercise & Session & $\mathbf{N}$ & Mean & Std. & Mean rank \\
\hline \multirow[t]{7}{*}{ Exercise 1} & 1 & 19 & 2.55 & .74 & 95.47 \\
\hline & 2 & 40 & 2.40 & .95 & 89.10 \\
\hline & 3 & 38 & 2.46 & .92 & 91.84 \\
\hline & 4 & 25 & 2.46 & .83 & 90.78 \\
\hline & 5 & 24 & 2.75 & .78 & 106.88 \\
\hline & 6 & 44 & 2.61 & .92 & 100.97 \\
\hline & Total & 190 & 2.53 & .88 & \\
\hline \multirow[t]{7}{*}{ Exercise 2} & 1 & 19 & 2.71 & .89 & 98.74 \\
\hline & 2 & 40 & 2.40 & .70 & 75.98 \\
\hline & 3 & 38 & 2.71 & .84 & 98.84 \\
\hline & 4 & 25 & 2.68 & .79 & 96.18 \\
\hline & 5 & 24 & 2.96 & .72 & 113.29 \\
\hline & 6 & 44 & 2.74 & .82 & 98.88 \\
\hline & Total & 190 & 2.68 & .80 & \\
\hline
\end{tabular}

Starting the analysis, we looked at the directions of measures within each session. Table 4.19 presents the descriptive statistics, comparing the measure means between the group using the tool and the group without the tool in the two exercises. Overall, the directions 
of measures were in line with our expectation in the two hypotheses. Almost all treatment groups had higher means than the control groups. One exception occurred in session 4 regarding exercise 1 where the treatment group had a lower mean than the other group. For all sessions (the last row of Table 4.19), the integrated means are consistent with the hypothesis directions.

Table 4.19. Comparison between groups using the tool and without the tool

\begin{tabular}{|c|c|c|c|c|}
\hline & \multicolumn{2}{|c|}{ Exercise 1} & \multicolumn{2}{|c|}{$\begin{array}{c}\text { Exercise } 2 \\
\text { (Swapped the group role) }\end{array}$} \\
\hline & $\begin{array}{r}\text { Group } \mathbf{A}- \\
\text { Without tool } \\
\text { Mean } \\
\text { (Std.) }\end{array}$ & $\begin{array}{r}\text { Group B - } \\
\text { Using the tool } \\
\text { Mean } \\
(\text { Std. })\end{array}$ & $\begin{array}{r}\text { Group A - } \\
\text { Using the tool } \\
\text { Mean } \\
\text { (Std.) }\end{array}$ & $\begin{array}{r}\text { Group } \text { B - } \\
\text { Without tool } \\
\text { Mean } \\
\text { (Std.) }\end{array}$ \\
\hline \multirow{2}{*}{ Session 1} & 2.35 & 2.78 & 2.85 & 2.56 \\
\hline & $(0.85)$ & $(0.57)$ & $(0.88)$ & $(0.92)$ \\
\hline \multirow{2}{*}{ Session 2} & 2.34 & 2.47 & 2.71 & 2.03 \\
\hline & $(1.07)$ & $(0.79)$ & $(0.55)$ & $(0.70)$ \\
\hline \multirow{2}{*}{ Session 3} & 2.16 & 2.73 & 2.89 & 2.55 \\
\hline & $(0.84)$ & $(0.92)$ & $(0.78)$ & $(0.89)$ \\
\hline \multirow{2}{*}{ Session 4} & 2.54 & 2.39 & 3.00 & 2.39 \\
\hline & $(0.78)$ & $(0.89)$ & $(0.60)$ & $(0.85)$ \\
\hline \multirow{2}{*}{ Session 5} & 2.57 & 3.06 & 3.23 & 2.50 \\
\hline & $(0.75)$ & $(0.77)$ & $(0.68)$ & $(0.56)$ \\
\hline \multirow{2}{*}{ Session 6} & 2.48 & 2.75 & 3.25 & 2.22 \\
\hline & $(0.88)$ & $(0.97)$ & $(0.67)$ & $(0.63)$ \\
\hline \multirow{2}{*}{ All sessions } & 2.40 & 2.67 & 2.99 & 2.34 \\
\hline & $(0.87)$ & $(\mathbf{0 . 8 6})$ & $(0.70)$ & $(0.77)$ \\
\hline
\end{tabular}

To use integrated data from these sessions, we first checked the potential differences of the scores among the sessions. As our sample datasets were not normally distributed, we used the non-parametric Kruskal-Wallis tests, which are an accepted alternative to ANOVA in case the datasets come from non-normally distributed population (Soh, Markus, \& Goh, 2006). The results of the Kruskal-Wallis tests showed that there were no significant differences among the six sessions for both exercise $1(p$-value $=0.788)$ and exercise 2 ( $p$-value $=0.145)$ at the 0.05 level. These results allowed us to analyse the datasets in an integrated way.

Using the integrated dataset, we tested the hypotheses H1 and H2. We chose MannWhitney tests to compare the different performance between the treatment and control groups because first, the tests were appropriate to the non-normally distributed population of the performance scores (Anderson, Sweeney, \& Williams, 2011; Pfeiffer et al., 2014). 
Second, the discontinuous measures used in the study called for the use of non-parametric tests, which might lead to having higher power compared to parametric tests (Soh et al., 2006). Finally, the distribution-free nature of the Mann-Whitney tests placed few restrictions on the dataset, and thus allowed us to analyse the dataset integrated from six experimental sessions. A similar use of the Mann-Whitney tests for analysing integrated datasets was reported by others, such as Mendling et al. (2012).

For each exercise, the Mann-Whitney tests were applied to the integrated dataset. We ran the tests using SPSS version 23.0. In this SPSS version, the software provided two ways to perform the Mann-Whitney tests: traditional (Legacy Dialogs) and new procedure (Nonparametric tests for independent sample). While the traditional procedure assumed the treatment and control samples had a similar-shape distribution, the new procedure actually tested this assumption. As the new procedure provided a more comprehensive test, we adopted and ran it on our integrated dataset. Regarding to exercise 1, the results of the Mann-Whitney tests are presented in Table 4.20. The distributions of the two groups are graphically shown in Figure 4.18.

Table 4.20. Results of Mann-Whitney tests on exercise 1

\begin{tabular}{|c|r|r|r|r|r|r|r|r|c|}
\hline \multirow{2}{*}{ Exercise 1 } & \multirow{2}{*}{ p-value } & \multicolumn{3}{|c|}{ Group A -Without tool } & \multicolumn{3}{|c|}{ Group B - Using the tool } \\
\cline { 5 - 9 } & & N & Mean & Std. & $\begin{array}{c}\text { Mean } \\
\text { Rank }\end{array}$ & N & Mean & Std. & $\begin{array}{c}\text { Mean } \\
\text { Rank }\end{array}$ \\
\hline Solution score & $\begin{array}{r}0.03 \\
(0.03)\end{array}$ & 99 & 2.40 & 0.87 & 87.44 & 91 & 2.67 & 0.86 & 104.27 \\
\hline
\end{tabular}

Note: the p-value of t-test is shown in parentheses for comparison purpose.

Independent-Samples Mann-Whitney U Test

\section{Group}

A

B

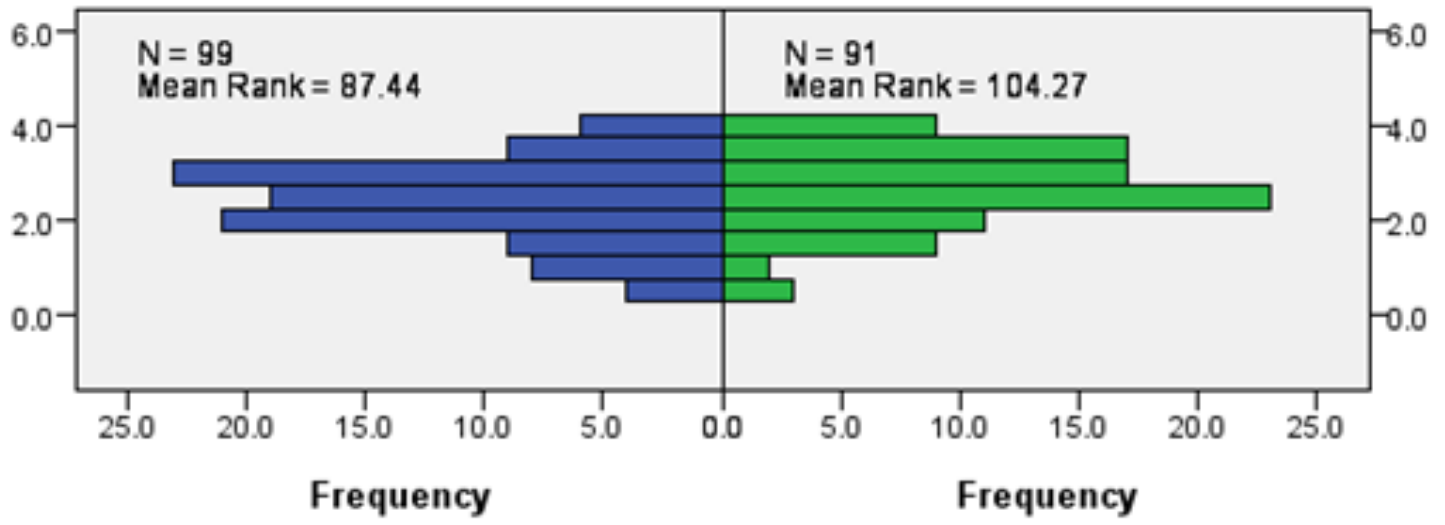

Figure 4.18. Distributions of Group A and Group B: Exercise 1 
Regarding exercise 2, the results of the Mann-Whitney tests are presented in Table 4.21. The distributions of the two groups are graphically shown in Figure 4.19. We note the swapping roles of the two groups, group A using the tool and group B without the tool.

Table 4.21. Results of Mann-Whitney tests on exercise 2

\begin{tabular}{|c|r|r|r|r|r|r|r|r|c|}
\hline \multirow{2}{*}{ Exercise 2 } & \multirow{2}{*}{ p-value } & \multicolumn{3}{|c|}{ Group A - Using the tool } & \multicolumn{4}{|c|}{ Group B -Without tool } \\
\cline { 5 - 9 } & & $\mathbf{N}$ & Mean & Std. & $\begin{array}{c}\text { Mean } \\
\text { Rank }\end{array}$ & N & Mean & Std. & $\begin{array}{c}\text { Mean } \\
\text { Rank }\end{array}$ \\
\hline Solution score & $\begin{array}{r}<0.001 \\
(<0.001)\end{array}$ & 99 & 2.99 & 0.70 & 116.62 & 91 & 2.34 & 0.07 & 72.53 \\
\hline
\end{tabular}

Note: the p-value of t-test is shown in parentheses for comparison.

\section{Independent-Samples Mann-Whitney U Test}

Group

A $\quad$ B

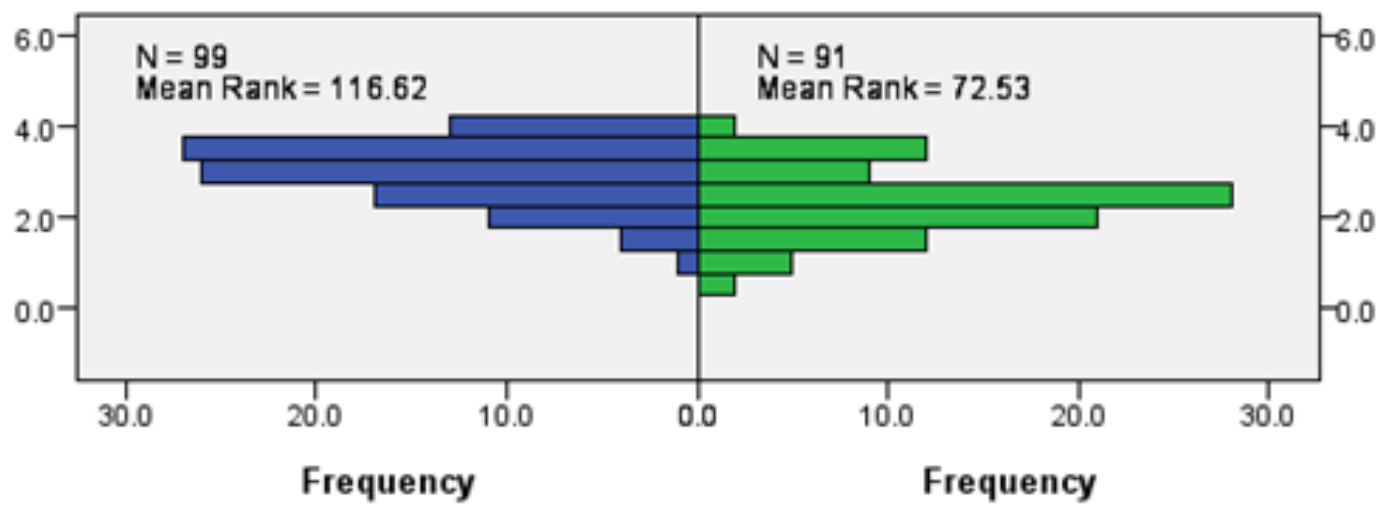

Figure 4.19. Distributions of Group A and Group B: Exercise 2

The experimental results shown in Table 4.20 and Table 4.21 support the hypotheses $\mathrm{H} 1$ and H2. More precisely, the results show that the performance of the treatment groups were indeed higher than the control groups (mean rank $=104.27$ vs. 87.44 regarding exercise 1, and 116.62 vs. 72.53 regarding exercise 2). Furthermore, the results confirm that the differences are significant at a 0.05 level in both exercise 1 ( $p$-value $=0.03$ and $U=5,302.5)$ and exercise 2 ( $p$-value $<0.001$ and $U=2,414.0)$. From these results, we suggest to accept both hypotheses. In other words, the tool can help improve the participants' performance on BPC decisions. When comparing between the two p-values, we note that although the two hypotheses are both statistically supported, the support to accept $\mathrm{H} 2$ is stronger than $\mathrm{H} 1$, which will be further discussed in relation to the focus group evaluation. In summary, we conclude that the two tools improve the BPC decisionmaking performance. 
Besides the performance analysis that showed the utility of the tool, we also analysed the tool usefulness perceived by the participants. We did this by analysing the survey data collected at the end of the experiments. The survey consisted of four questions rating the perceived usefulness (PU1 - PU4). Of the 190 participants, 181 completed the survey. Table 4.22 shows the statistics of the survey results.

Table 4.22. $\quad$ Statistics of usefulness perceived by experimental participants

\begin{tabular}{|l|r|r|}
\hline \multicolumn{1}{|c|}{ Perceived Usefulness } & \multicolumn{1}{|c|}{ Mean } & \multicolumn{1}{|c|}{ Std. } \\
\hline $\begin{array}{l}\text { PU1 - Using the tool allows me to better answer the questions } \\
\text { in the exercises }\end{array}$ & 3.98 & .632 \\
\hline $\begin{array}{l}\text { PU2 - Using the tool allows me to faster answer the questions } \\
\text { in the exercises }\end{array}$ & 4.01 & .796 \\
\hline $\begin{array}{l}\text { PU3 - Using the tool allows me to better understand the } \\
\text { questions in the exercises }\end{array}$ & 3.96 & .766 \\
\hline PU4 - I find using the tool useful & 4.28 & .667 \\
\hline
\end{tabular}

Note: We used 1-5 scale to rate the PUs where 1 is useless and 5 is useful.

The results show that all items display a tendency towards perceived usefulness, i.e. all means $>3.9$ on the 1-to-5 scale that varied from extreme uselessness (1) to extreme usefulness (5). In other words, the participants perceive the tool to be useful for their tasks. However, we note that the perceived usefulness here needs to be interpreted carefully. This is because when we examine the dataset of participants who did not perform well when using the tool (scoring less than or equal to 2 on the 0 -to- 4 scale), their perceived usefulness is still high (mean of 4.07). This indicates that the participants might answer the survey without considering the tool performance. An explanation for this is that the participants know the researcher is also a lecturer in the university, leading to a potential bias when students evaluate their instructors (Marsh, 2007).

\section{Validity}

To strengthen the knowledge claims from the experiments, we had to identify and handle several threats to validity (Wohlin et al., 2012). Table 4.23 presents those that were most relevant to the experiments, and summarises how we handled them. 


\section{Table 4.23. Validity of the experiments}

Conclusion validity: concerns issues that may influence the correctness of the conclusion. Two aspects were focused: the use of appropriate statistical tests and avoiding irrelevancies in the experimental setting.

- We screened data for their appropriateness with the statistical tests. As the normality assumption did not hold, we used Mann-Whitney tests to analyse the difference between the control and treatment groups.

- We further analysed the possible influence of other factors like participants' background, gender, and year of study on the results. Both the chi-square tests and Mann-Whitney tests rejected hypotheses that the performance was different across different categories of background, gender, and year of study. Consequently, these results reduced the threads of irrelevant factors influencing the results.

Internal validity: requires causal relationship between treatment and outcome.

- We randomly assigned participants to the control and treatment groups

- To avoid learning effect, we swapped group roles in exercise 2 (the control group became the treatment group, and vice versa).

- This validity also related to the dropout rate. In the pilot experiments, some participants did not use the tool to address tasks, leading to a small rate of dropout in the treatment group. The main experiments handled this issue by asking the participants to save their answers, which increased their interaction with the tool and thus minimised the dropout rate.

Construct validity: concerns how the measures represent their theoretical basic.

- To measure the performance, we used two exercises consisting of four questions, which were developed based on previous studies (Afuah \& Tucci, 2012; Kittur et al., 2013). Yet, we noted the exploratory nature of our study, which suggests that the constructs still need to be further analysed and tested.

External validity: concerns the generalizability level of the results

- Kruskal-Wallis tests were particularly motivated by external validity considerations. The tests confirmed that there were no significant differences between the six sessions, thereby increasing the external validity.

- As noted earlier, using students as proxies for crowdsourcing practitioners might threat an external validity. However, as the tools proved to be helpful for students with less crowdsourcing experience, they would certainly be helpful for crowdsourcing practitioners. Furthermore, Höst et al. (2000), who compared between students and professionals as experimental subjects in software engineering, found very minor differences between the two groups. 
In summary, we conducted experiments to evaluate the decision tool supporting BPC establishment. More precisely, we examined how much the tool helped making informed decisions for the scenario applications. We designed the experiments where the treatment groups used the tool and the control groups did not use the tool to address the same exercises. Two hypotheses were tested on using the tool to support the decision to crowdsource (H1) and the process design (H2). Six experimental sessions with 190 participants were conducted, which together formed an integrated dataset of the experiments. Since the dataset was not normally distributed, we used non-parametric tests to analyse the data. The results provide support for both hypotheses, which suggests that the use of the tool (comprising of Tool 1 and Tool 2) leads to better performance on BPC decisions. In short, the tool is useful in supporting BPC establishment.

\subsubsection{Evaluation: Focus Group}

\subsubsection{Overview of the Approach}

The previous section has already evaluated the tool through controlled experiments, which can have precision, but are not very strong in exploring the participants' perception. To examine the tool usefulness perceived by the participants, this section presents the focus group approach to evaluate the tool. While the justification for using focus groups has been discussed in Chapter 3, here we highlight how complementary the focus groups are to the above experiments, by examining two aspects. First, focus groups provide qualitative evaluation (Krueger \& Casey, 2014; Tremblay et al., 2012), complementary to the quantitative data of the experiments. Second, focus groups, having the strength of group discussion, can give us key interaction-based insights that may not surface in the experiments.

Adopting the focus group approach, our aim was to gather qualitative evidence of the tool's utility. This aim identified the nature of the focus group. Tremblay et al. (2010) categorised focus groups being used in design science into exploratory ones that generated design features of artefacts, and confirmatory ones that gathered evidences of the artefact utility. Considering our aim, the confirmatory focus groups were adopted. More precisely, we used the focus groups to confirm the tool utility perceived by the participants, regarding three aspects: 1) perceived usefulness that measures 'what ways 
the tool can contribute to BPC establishment'; 2) perceived ease of use that measures 'the ability that the users can comprehend the tools to perform their targeted tasks'; and 3) possible improvements of the tool.

\subsubsection{Focus Group Design}

To guide the focus group design, we adapted the procedure of how to conduct focus groups in design science proposed by Tremblay et al. (2010). Figure 4.20 presents a summary of the adapted procedure, consisting of problem formulation, sample frame and moderator, question route development, conduct of focus group, and data analysis. Besides the problem formulation that was already presented in the previous section, the rest of the procedure is presented in this section.

\section{Formulate Research Problem}

- Confirmatory focus groups to evaluate the decision tool, regarding perceived usefulness, perceived easy to use, \& possible improvements

\section{Identify Sample Frame \& Moderator}

- Number of groups: 2 confirmatory focus groups

- Size of groups: 4 to 6 participants

- Recruitment: Crowdsourcing experts \& PhD students with related research focus

- Moderator: the researcher plays the role of the focus group moderator

Plan focus group agenda

\section{Develop a Questioning Route}

- Develop questions directing the discussion

\section{Conduct Focus Group}

- There were three sections in the focus group

- Introduce the decision tool

- Make crowdsourcing decision without and with the tool

- Group discussion

\section{Analyse and Interpret Data}

- Transcribe focus group discussion

- Coding with the three predefined measures

- Report main themes

Figure 4.20. Focus group procedure (adapted from Tremblay et al., 2010) 


\section{Sample Frame and Moderator}

Four key decisions were made in this stage: the number of focus groups, the desired number of participants in each group, recruitment participants, and moderator identification. The literature was not clear about the number of groups necessary to evaluate IS artefacts. For instance, Gibson and Arnott (2007) used one focus group to evaluate a business intelligence system, while Miah et al. (2009) conducted three focus groups to acquire knowledge backing the design artefact. More recently, Tremblay et al. (2010) suggested that focus groups for design science should include one pilot, two exploratory, and two confirmatory groups. Considering the suggestion, combining with the confirmatory nature of our evaluation, the current study conducted two focus groups. Regarding group size, focus groups normally include a range from four to twelve participants. Within this range, Tremblay et al. (2010) explain some trade-offs between smaller and larger group sizes. For instance, smaller groups require group members to participate more, while larger groups increase complexity. These authors explicitly suggest using about six participants as "large focus groups (more than six) could be tricky in design research since the subject matter is more complex than traditional focus group topics" (Tremblay et al., 2010, p. 603). Following this suggestion, we recruited one group with four participants and the other group with six participants.

Regarding the nature of the two groups, we recruited one with diverse crowdsourcing backgrounds for assessing different perspectives of the tool, and one with homogeneous crowdsourcing experts for thorough assessing the tool. In the first group, we recruited four $\mathrm{PhD}$ students with backgrounds covering crowdsourcing, social media, social network, and IS quality evaluation. This group helped assess the tool from the view of general users, who in practice might come to the tool with less crowdsourcing background. Furthermore, it served as a pilot test on the script, setting, and question route of the focus groups. The second group recruited six crowdsourcing practitioners and researchers, who had more than one year of crowdsourcing experience. The aim of this focus group was to thoroughly evaluate the tool from the view of crowdsourcing experts. Table 4.24 and Table 4.25 show demographic characteristics of the focus group samples. 
Table 4.24. $\quad$ Sample offocus group 1: PhD students

\begin{tabular}{|l|l|l|l|l|l|}
\hline Gender & Age & $\begin{array}{c}\text { Crowdsourcing } \\
\text { expertise }\end{array}$ & $\begin{array}{c}\text { Current } \\
\text { position/ } \\
\text { occupation }\end{array}$ & $\begin{array}{r}\text { Years of } \\
\text { crowdsourcing } \\
\text { experience }\end{array}$ & Research focus \\
\hline Female & $25-34$ & Researcher & PhD student & $<6$ month & Crowdsourcing \\
\hline Male & $25-34$ & $\begin{array}{l}\text { Interested in } \\
\text { crowdsourcing }\end{array}$ & PhD student & $<6$ month & Social network \\
\hline Male & $25-34$ & $\begin{array}{l}\text { Interested in } \\
\text { crowdsourcing }\end{array}$ & PhD student & $<6$ month & $\begin{array}{l}\text { IS service } \\
\text { evaluation }\end{array}$ \\
\hline Female & $25-34$ & $\begin{array}{l}\text { Interested in } \\
\text { crowdsourcing }\end{array}$ & $\begin{array}{l}\text { PhD student } \\
\text { Lecturer }\end{array}$ & $<6$ month & Social media \\
\hline
\end{tabular}

Table 4.25. $\quad$ Sample offocus group 2: Crowdsourcing experts

\begin{tabular}{|l|l|l|l|l|l|}
\hline Gender & Age & $\begin{array}{c}\text { Crowdsourcing } \\
\text { expertise }\end{array}$ & $\begin{array}{c}\text { Current } \\
\text { position/ } \\
\text { occupation }\end{array}$ & $\begin{array}{c}\text { Years of } \\
\text { crowdsourcing } \\
\text { experience }\end{array}$ & $\begin{array}{l}\text { Years of work } \\
\text { experience }\end{array}$ \\
\hline Male & $45-54$ & $\begin{array}{l}\text { Practitioner/ } \\
\text { Researcher }\end{array}$ & $\begin{array}{l}\text { Associate } \\
\text { director library } \\
\text { technology } \\
\text { service }\end{array}$ & $>2$ years & $>5$ years \\
\hline Female & $>55$ & Practitioner & Contractor & $>2$ years & $>5$ years \\
\hline Male & $25-34$ & Researcher & & $1-2$ years & $1-2$ years \\
\hline Male & $>55$ & Researcher & Senior Lecturer & $>2$ years & $>5$ years \\
\hline Male & $35-44$ & Practitioner & $\begin{array}{l}\text { Platform owner } \\
\text { Digital }\end{array}$ & $>2$ years & $>5$ years \\
collections & Lecturer & $1-2$ years & $>5$ years \\
\hline Female & $>55$ & Researcher & Lears &
\end{tabular}

As focus groups need to be moderated, the researcher acted as a moderator of the focus groups. This role included introducing the decision tool to the participants, facilitating the discussion, and dealing with the dynamic in the group discussion. As the research was also the artefact designer, the researcher came to the moderator role with an open mind regarding the tool evaluation. That is, the researcher viewed the focus group as a good opportunity to receive suggestions for improvements, constructive feedback and (sometimes) criticism. During the focus group, the moderator sometimes answered questions on how to use the tool, as participants could not be expected to be completely familiar with the tool functionality after just a short introduction. 


\section{Questioning Route and Crowdsourcing Scenario Development}

Before the focus groups, we developed a questioning route, which would set the direction of the group discussion. The questioning route included ten questions used by the moderator to initiate the discussion. Due to the pilot role of the first focus group, these questions were slightly revised in the second focus group. A partial questioning route is presented in Appendix G.

\section{Focus Group Conduct}

The focus groups were held in a meeting room at the School of Information Management, Victoria University of Wellington. The room had several laptops accessing the decision tool. After the welcome, the moderator introduced the tool functionality to help the participants become familiar with the tool. Then, the moderator delivered two scenarios to the participants, each of which included a short description and five decisions related to the decision to crowdsource and process design. These scenarios, which were developed based on the two case studies in Section 4.2.2, ensured the participants used the tool and thus enabled them to discuss the tool. The moderator encouraged the participants played the role of a crowdsourcing decision maker addressing these scenarios. At the beginning, the participants were asked to make the decisions on the related BPC aspects without the tool. Then, they were asked to access the decision tool and reconsider their decisions using the tool. They were prompted to write out both their initial decisions and revised decisions (if there were any).

The ensuing discussion revolved around how the decision tool was used and its ability to support the decision-making process. Starting the discussion, the moderator asked some initial questions about the tool characteristics, and then allowed the discussion to flow. The discussion were audio recorded and transcribed. At the end of the discussion, both the initial and revised decisions of the participants were handed to the researcher. Some notes were also taken by the moderator during the discussion. All these activities took about 1.5 hours for each focus group.

\section{Analysis}

For data analysis of design science focus groups, Tremblay et al. (2010) note several qualitative analysis techniques that can be used and highlight the use of template analysis due to its flexible and simple procedure. Template analysis is referred to as "thematic 
analysis that balances a relatively high degree of structure in the process of analysing textual data with the flexibility to adapt it to a need of a particular study" (King, 2012, p. 426). Following Tremblay et al.'s (2010) suggestion, we adopted the template analysis and further viewed the technique as appropriate for two reasons. Our focus groups evaluated the tool with three pre-defined measures: perceived usefulness, ease of use, and possible improvements, while one distinctive characteristic of template analysis is the use of pre-defined themes (Brooks, McCluskey, Turley, \& King, 2015). Furthermore, this analysis technique can be used within the design science paradigm, as suggested by Tremblay et al. (2012).

Adopting the template analysis, we developed the initial templates based on the three measures and some lower codes focusing on some aspects of the measures. A code is defined as "a label attached to a section of text to index it as relating to a theme or issue in the data which the researcher has identified as important to his or her interpretation" (King, 2004, p. 257). We then applied the codes to the transcribed focus group discussions. More precisely, we reviewed the transcripts and identified sections of the text relevant to our codes. During this process, we created some other codes to explore the entire range of the discussion. Table 4.26 presents an example of the coding schema. The codes were then aggregated into categories and themes.

Table 4.26. Example of coding schema

\begin{tabular}{|l|l|l|}
\hline \multicolumn{1}{|c|}{ Measure } & \multicolumn{1}{|c|}{ Code } & \multicolumn{1}{c|}{ Definition } \\
\hline $\begin{array}{l}\text { Perceived } \\
\text { usefulness }\end{array}$ & Decision framing & $\begin{array}{l}\text { The ability to frame crowdsourcing } \\
\text { decisions, which may lead to change in these } \\
\text { decisions prior and after using the tool }\end{array}$ \\
\cline { 2 - 3 } & Additional information & $\begin{array}{l}\text { The complementary information provided by } \\
\text { the tool, in addition to what the participants } \\
\text { had already known without the tool }\end{array}$ \\
\hline $\begin{array}{l}\text { Perceived ease } \\
\text { of use }\end{array}$ & Ease of use & $\begin{array}{l}\text { Participants can comprehend the tools to } \\
\text { perform their targeted tasks }\end{array}$ \\
\hline $\begin{array}{l}\text { Possible } \\
\text { improvement }\end{array}$ & Knowledge improvement & $\begin{array}{l}\text { Suggestion to improve the tool regarding } \\
\text { crowdsourcing information, factors, advice, } \\
\text { and decisions }\end{array}$ \\
\cline { 2 - 3 } & Technical improvement & $\begin{array}{l}\text { Suggestion to improve the tool regarding } \\
\text { user interface and technical functions }\end{array}$ \\
\hline
\end{tabular}




\subsubsection{Focus Group Results}

The results of the focus groups are structured according to the three investigated measures: perceived usefulness, perceived ease of use, and possible improvements. Regarding the first, perceived usefulness was further analysed with two aspects: the ability of the tool to provide additional and structured information to the participants, and the ability to change the participants' decisions after using the tool. For this analysis, the two codes 'additional information' and 'Decision framing' were examined. In general, there were mixed results regarding the two aspects. On the one hand, we found that the decision tool provided additional structured information for making the decisions, as demonstrated by the following comments:

"It is definitely promoting a lot of the right things to help make a correct decision."

"The tool makes it clear why it was saying not to do [crowdsourcing], so you feel confident, it was an informed decision."

"The tool provides more concrete [information]. I have some abstract ideas, it helps the actually specifics."

Further analysing this aspect, we found quite a common template that the tool reminded the participants of something that they forgot, which highlighted the ability of the tool for providing structured information related to BPC.

"It forces me to think about the risk which I haven't thought about when I did it [crowdsourcing]."

"With the tool, it brought up some privacy issues."

"I think for me, this is absolutely helpful to say have you thought about this."

On the other hand, the supporting evidence for framing decisions was not strong. In other words, the results were mixed regarding how the tool could frame crowdsourcing decisions and change the participants' decisions. Some participants changed at least one decision as a result of using the tool. Reflecting that, when being asked whether they changed their decision after using the tool, some participants wrote out:

"Yes, quality aspects have changed, which is good to point out."

"Yes, the decision to crowdsourcing function [of the tool] influenced me." 
"Yes, [I] have added the role of the internal experts and have stated that this is a complex project."

However, other participants did not change their decisions. In some cases, the participants' thought covered the tool's framing, as seen via "the tool provides advice that follows my own understanding”. In a few cases, even though the tool suggested different decisions, the users still kept their own decisions. As one of them stated that "I still rely on my own decision making".

Regarding perceived ease of use, most participants made very similar comments and suggested that the tool was clear and understandable. For example, one of them commented: "Yes, the tool is easy to use and I don't have any difficulty to learn it". This aspect was also confirmed by the fact that all the participants learned how to use the tool through a short introduction in the focus groups. They mastered the tool quickly, except one participant who needed further explanation about the tool's navigation during the focus group.

Finally, we also analysed the possible improvements for the tool, including knowledge improvements and technical improvements. The focus groups suggested a few knowledge improvements. Some participants suggested adding more decision factors and design issues that should be considered in the tools, including confidentiality, sustainability, timeline, life cycle, whether tasks can be automatic, and crowd engagement. For instance, one participant recommended that the decision to crowdsource should examine the confidentiality of the crowd. We note that, on the one hand, these suggested factors and issues would be interesting to explore further with the possibility of the tool revisions. On the other hand, as these factors and issues were not suggested by the 'wisdom of researchers' in our knowledge sources analysis (Section 4.1), they might not be key factors for different crowdsourcing contexts. Thus, the suggested factors and issues should be further examined before possibly including or excluding them into our tool.

Another interesting suggestion was to give different weights to the decision factors and issues, and ultimately to use these weights for aggregating and generating the final decision. One participant stated "the idea of weighting is interesting too, where some of these issues are perhaps more important than others". Although such a weighting approach was not included in the tool yet, it was aligned with the nature of the tool. That 
is, the tool was developed based on the 'wisdom of researchers' and thus the numbers of the supporting sources could be used as weights.

During the discussion, some technical improvements were also suggested. We noted that some suggestions were not actual problems after some explanation and discussion. For instance, a participant commented on why the tool did not immediately move to the next question after the user answered a question. The explanation was that after the user answered a particular question, the tool needed to show advice for the current question, and thus needed not to move to the next question. Besides that, other suggestions might improve the tool, and thus they should be further considered. Examples included a confusion where two issues in the design tool had the same headings; and some questions, pre-defined answers, and advice needed to be clarified, e.g. to provide more examples to clarify the questions and pre-defined answers.

Overall, the focus group results were positive towards the tool utility. Table 4.27 summarises the main evaluation findings of the focus groups. As seen in Table 4.27, we find clear evidence of perceived usefulness that the tool can provide additional structured information related to BPC decisions. It is also evidence of perceived ease of use. Yet, evidence of decision framing that the tool can change participants' decisions is mixed. Further, some possible improvements for the tool are also noted. In summary, we suggest that the tool provides promising support to BPC decisions though some improvements are desired.

Table 4.27. Summary of focus group results

\begin{tabular}{|l|l|l|c|}
\hline $\begin{array}{l}\text { Focus } \\
\text { Group }\end{array}$ & Supporting evidence & Counter-evidence & $\begin{array}{c}\text { Evaluation results } \\
\text { (Evidence of utility) }\end{array}$ \\
\hline Perceived usefulness - Providing additional information & \multicolumn{2}{|l|}{ Yes } \\
\hline FG 1 & $\begin{array}{l}\text { Several instances where the } \\
\text { tool was useful to provide } \\
\text { information and structures for } \\
\text { the BPC decisions }\end{array}$ & None & Yes \\
\hline FG 2 & $\begin{array}{l}\text { A common template where } \\
\text { the tool reminded the } \\
\text { participants something that } \\
\text { they did not think of without } \\
\text { the tool }\end{array}$ & & \\
\hline
\end{tabular}




\begin{tabular}{|c|c|c|c|}
\hline \begin{tabular}{|l|} 
Focus \\
Group
\end{tabular} & Supporting evidence & Counter-evidence & $\begin{array}{l}\text { Evaluation results } \\
\text { (Evidence of utility) }\end{array}$ \\
\hline \multicolumn{4}{|c|}{ Perceived usefulness - Decision framing } \\
\hline FG 1 & $\begin{array}{l}\text { After using the tool to frame } \\
\text { crowdsourcing decisions, } \\
\text { some participants changed at } \\
\text { least one decision }\end{array}$ & $\begin{array}{l}\text { A few participants made } \\
\text { a decision on their own } \\
\text { knowledge }\end{array}$ & Mixed \\
\hline FG 2 & $\begin{array}{l}\text { After using the tool to frame } \\
\text { crowdsourcing decisions, } \\
\text { some participants changed at } \\
\text { least one decision }\end{array}$ & $\begin{array}{l}\text { Some participants } \\
\text { suggested the tool did not } \\
\text { change their decisions }\end{array}$ & Mixed \\
\hline \multicolumn{4}{|c|}{ Perceived easy to use } \\
\hline FG 1 & $\begin{array}{l}\text { Most participants suggested } \\
\text { the tool was easy to } \\
\text { understand }\end{array}$ & None & Yes \\
\hline FG 2 & $\begin{array}{l}\text { Most participants suggested } \\
\text { the tool was easy to } \\
\text { understand }\end{array}$ & $\begin{array}{l}\text { One participant needed } \\
\text { support on how to use the } \\
\text { tool }\end{array}$ & Yes \\
\hline \multicolumn{4}{|c|}{ Possible improvement - Knowledge improvement } \\
\hline FG 1 & & $\begin{array}{l}\text { A few suggestions for } \\
\text { knowledge } \\
\text { improvements }\end{array}$ & $\begin{array}{c}\text { High } \\
\text { (a few suggested } \\
\text { improvements) } \\
\end{array}$ \\
\hline FG 2 & & $\begin{array}{l}\text { Some suggestions for } \\
\text { additional decision } \\
\text { factors/design issues, and } \\
\text { for weighting them }\end{array}$ & $\begin{array}{c}\text { Average } \\
\text { (some suggested } \\
\text { improvements) }\end{array}$ \\
\hline \multicolumn{4}{|c|}{ Possible improvement - Technical improvement } \\
\hline FG 1 & & $\begin{array}{l}\text { Some suggestions for } \\
\text { technical improvements }\end{array}$ & $\begin{array}{c}\text { Average } \\
\text { (some suggested } \\
\text { improvements) } \\
\end{array}$ \\
\hline FG 2 & & $\begin{array}{l}\text { Some suggestions for } \\
\text { technical improvements }\end{array}$ & $\begin{array}{c}\text { Average } \\
\text { (some suggested } \\
\text { improvements) } \\
\end{array}$ \\
\hline
\end{tabular}

Before concluding the section, we recall a note from the experiment results. That is, the experimental results supported the utility of both main functions of the tool (Tool 1 and Tool 2), yet the statistical support of Tool 2 was stronger than Tool 1. This note can now be explained with the focus group results. To clarify, Tool 1 focuses on the decision to crowdsource, which is a go/no-go decision. The focus group results show that this type of decision is quite hard to change (mixed results on decision framing), which is aligned with the equal support of the experimental results for the Tool 1's utility. Differently, 
Tool 2 focuses on the BPC design issues. The focus group results show that providing structured information is largely helpful for these issues (strong results that the tool can provide structured information). This possibly explains why the experimental results strongly supports the Tool 2's utility.

In conclusion, the focus group results provide qualitative evaluation on the tool, complementary to the experimental evaluation. The focus groups generate rich discussion and assessment the tool from different angles. These rich data help point out what areas the tool can make contributions and what still need to be improved. As a result, the qualitative evaluation here, together with the quantitative evaluation from the experiments, strengthens the confidence on the utility of the decision tool.

\subsubsection{Summary and Remarks}

This stage constructed a decision tool supporting BPC establishment, operationalising the ontological knowledge base developed in the previous stage. Considering the complexity of the construction, we developed a tool architecture and adopted a rapid prototyping method (Kordon, 2002; Lim et al., 2008). The tool architecture consisted of three main modules: GUI, information-processing module, and a knowledge module that was backed by the BPC ontology (Section 4.3). We used the architecture to develop two prototypes. While the first prototype enabled us to test the tool for revision feedback, the second one was targeted to support the project managers and process designers, making informed decisions in BPC establishment.

The decision tool was carefully evaluated. We assessed the tool using experiments as quantitative evaluation and focus groups as qualitative evaluation. In the experiments, 190 participants were asked to address two crowdsourcing exercises. They were allocated to the control groups without the tool and the treatment groups with the tool. The results find significantly higher performance of the treatment groups compared to the control groups in both exercises. This suggests the tool is useful in supporting BPC decisions.

To further evaluate the tool, two focus groups were conducted. One group consisted of crowdsourcing experts, and the other consisted of $\mathrm{PhD}$ students with the related backgrounds. In each focus group, the participants considered some BPC decisions without and then with the tool. Based on the interaction with the tool, they then discussed about the tool functionality and its support for making BPC decisions. The focus group 
results likely confirm the utility of the tool, especially regarding its ability to structure and provide useful information in establishing BPC.

The combination of the two evaluation techniques increases our confidence when suggesting the tool as a means for supporting BPC establishment.

\subsection{Chapter Summary}

This chapter has presented the main findings of the thesis, which were structured within the four stages of the research process. The first stage scoped knowledge sources in the BPC domain. The other stages built a set of three BPC artefacts: a conceptual model, ontology, and a decision support tool, and evaluated them using the case study, triangulation, experiments, and focus groups. These artefacts have contributed to move BPC establishment forward. The findings from these stages are discussed in the next chapter in order to answer the research questions. 


\section{INTEGRATED RESULTS}

The preceding chapter has presented the detailed findings of the research. This chapter examines them from a more integrated perspective, highlighting the interrelated nature of the research. The chapter starts with an overview of the findings. Next, it presents our consolidation of the findings for answering the research questions. Finally, a summary of the chapter is presented.

\subsection{Overall Results}

The research results have been formed from the four research stages. While the previous chapter presented the results as sequential stages' outcomes, these results are related due to the interrelated nature of the research. This section examines the results from an integrated viewpoint in order to provide an overall picture of the research outcomes. In particular, four major integrated outcomes are discussed.

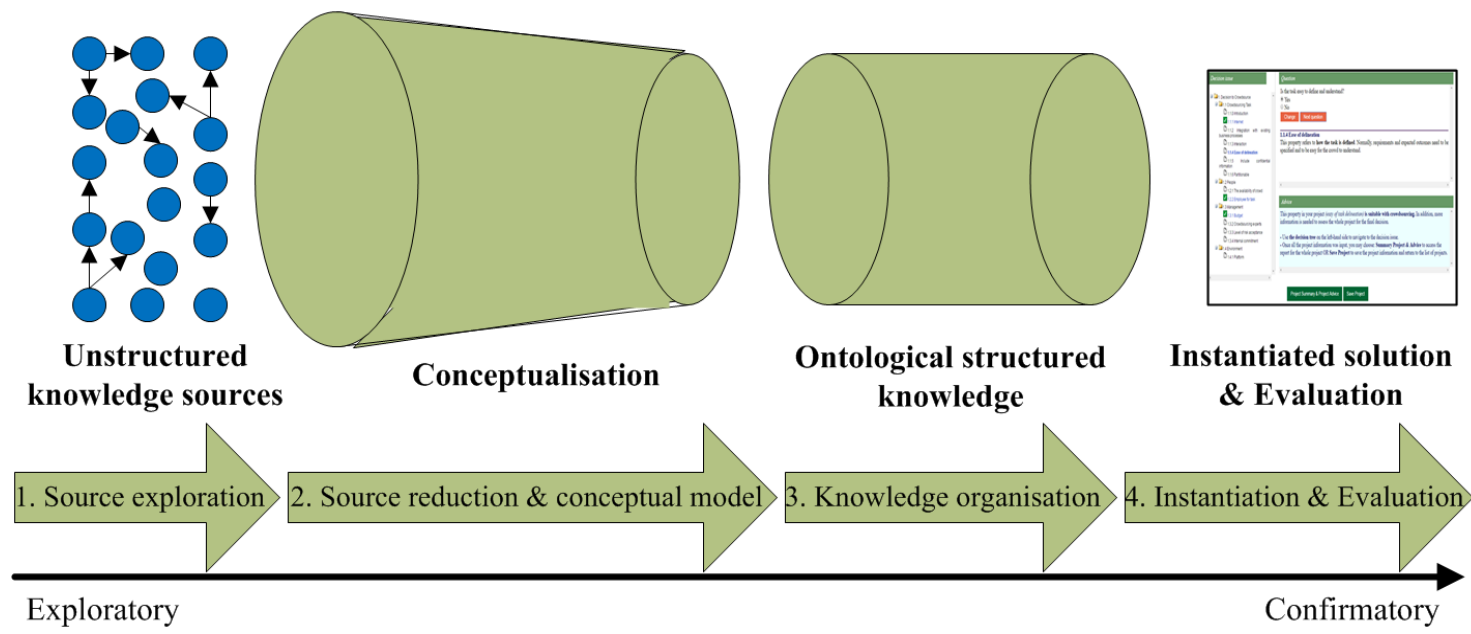

Figure 5.1. Interrelated knowledge in structuring BPC

First, we note that the research results are interrelated in structuring the BPC domain. This is because the four research stages together examine the BPC domain, aligning to the exploratory-confirmatory continuum suggested by Miles et al. (2014). Figure 5.1 illustrates this interrelation. The first stage explored the knowledge sources in the domain, which had not been structured before. The second stage deducted the knowledge sources and conceptualised the BPC concept. It offered a conceptual model that synthesised 
unstructured knowledge into the focused building blocks of BPC. The third stage, extending this conceptualisation, organised knowledge in the domain using an ontological structure. The final stage instantiated a decision tool founding on the ontological structure, and then evaluated and confirmed the tool utility. In reflecting through the research stages, BPC knowledge has been sequentially structured, starting from diverse unstructured knowledge sources, to abstract conceptualisation, to an ontological structure, and finally to the instantiated decision tool supporting BPC establishment. Consequently, we suggest that the research results enable different yet interrelated knowledge for structuring BPC.

Second, the research results also suggest interrelated yet different levels of abstraction for understanding BPC establishment. This difference allows us to speak both abstractly about managerial aspects of BPC, and more concretely about its building blocks and detailed processes. The conceptual model, ontology, and decision support tool form three levels of BPC abstraction, which are depicted in Figure 5.2. In the figure, the conceptual model presents abstract building blocks of BPC; the ontology specifies these building blocks into detailed elements, including processes, activities, data, and their relationships; and the decision tool operationalises these ontological elements with decision tables, what-if scenarios, and contextual recommendations. Given the three levels of abstraction, it is possible for different stakeholders to focus on different levels of concern but still reach consistency on BPC establishment. These consistent yet different levels of foci are an important requirement to establish complex business processes involving multiple stakeholders like BPC (Berente et al., 2009; Giachetti, 2004; Hasselbring, 2000).

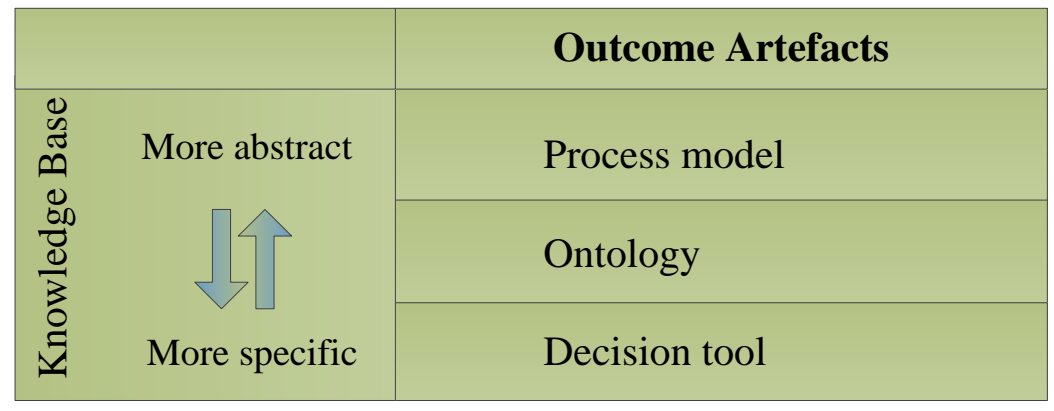

Figure 5.2. Interrelated yet different levels of abstraction: Outcome artefacts

The first two outcomes lead to the third interrelated result, which is the ability to trace back the BPC knowledge through the research stages (the upward arrow of Figure 5.2). That is, operationalised knowledge in the decision tool can properly be traced back to the 
ontological elements, which can be mapped to the model components and in turn traced back to the knowledge sources. The traceability comes from the systematic approach brought by the design science research, where we systematically structure the research activities and explicitly justify and present key decisions made in these activities. This systematic approach is similar to the evidence-based strategy in design science (Denyer \& Tranfield, 2006; Van Aken, 2005; Van Aken \& Romme, 2012).

The fourth and final integrated outcome is the multiple iterations of build and evaluate activities in the research process. Inspired by the design cycle suggested by Hevner and Chatterjee (2010), we designed and then evaluated each artefact before moving to the next research stage. These iterations allow us to better understand the design problem, its solution, and how the solution addresses the problem through evaluation. The iterations also enhance the relevance and rigour of the research stages by continuous evaluating the outcome artefacts (Hevner \& Chatterjee, 2010; Sonnenberg \& vom Brocke, 2012b). As a result, the build-evaluate iterations strengthen the relevance and rigour of the entire research process and its generated BPC knowledge.

\subsection{Answering the Research Questions}

This section organises the research results from a thematic viewpoint in order to answer the research questions. Four research questions have guided the dissertation, which are recollected here.

1. RQ1: What are the main building blocks of BPC that can be identified in the domain?

2. RQ2: What are the structures of the identified building blocks for conceptualising $\mathrm{BPC}$ ?

3. RQ3: How to construct a domain ontology of BPC that organises the unstructured knowledge sources in the domain?

4. RQ4: How to construct a decision tool supporting organisations in establishing BPC?

Table 5.1 summarises the research results that answer the research questions. The table is structured around four columns. The first column shows the four stages of the design science research. Then, as design science highlights both design processes and design products, the second and third columns present the research activities and research outcomes respectively. The final column refers to the research questions being addressed. 
Table 5.1. Summary of results that answer the research questions

\begin{tabular}{|c|c|c|c|}
\hline $\begin{array}{l}\text { Research } \\
\text { Stage }\end{array}$ & Research Activity & Research Outcome & RQ \\
\hline $\begin{array}{l}\text { 1. Scoping } \\
\text { knowledge } \\
\text { sources }\end{array}$ & $\begin{array}{l}\text { BPC Knowledge Base } \\
\text { - Identified and analysed } 238 \\
\text { knowledge sources } \\
\text { - Synthesised BPC building blocks } \\
\text { supported by at least } 10 \text { sources } \\
\text { - Synthesised factors influencing } \\
\text { the decision to crowdsource }\end{array}$ & $\begin{array}{l}\text { - } 12 \text { building blocks of BPC } \\
\text { (Table 4.2) } \\
\text { - Additional outcomes: nine } \\
\text { factors and sixteen sub-factors } \\
\text { influencing the decision to } \\
\text { crowdsource (Table 4.3) }\end{array}$ & RQ1 \\
\hline \multirow[t]{2}{*}{$\begin{array}{l}\text { 2. Develop } \\
\text { the IS } \\
\text { Artefact }\end{array}$} & $\begin{array}{l}\text { Conceptual Model } \\
\text { - Synthesised the most salient BPC } \\
\text { building blocks } \\
\text { - Applied the analytic framework } \\
\text { to arrange the model components } \\
\text { - Defined the components } \\
\text { - Developed a framework } \\
\text { supporting the decision to } \\
\text { crowdsource }\end{array}$ & $\begin{array}{l}\text { - A process model of BPC } \\
\text { (Figure 4.3) } \\
\text { - } 3 \text { stages: decision to } \\
\text { crowdsource, design, and } \\
\text { configuration } \\
\text { - } 7 \text { components in the three } \\
\text { stages } \\
\text { - Additional outcomes: A } \\
\text { decision framework of the } \\
\text { decision to crowdsource } \\
\text { (Figure 4.4) }\end{array}$ & \multirow[t]{2}{*}{ RQ2 } \\
\hline & $\begin{array}{l}\text { Case Study Evaluation (two } \\
\text { crowdsourcing projects) } \\
\text { - Collected multiple data sources, } \\
\text { including interviews with key } \\
\text { informants } \\
\text { - Analysed the project activities, } \\
\text { using the model } \\
\text { - Analysed the utility of the model } \\
\text { perceived by the interviewees }\end{array}$ & $\begin{array}{l}\text { - High representation of the } \\
\text { projects' activities (Figure } 4.5 \\
\text { and Figure 4.6) } \\
\text { - Usefulness perceived by the } \\
\text { interviewees for planning and } \\
\text { running crowdsourcing } \\
\text { projects (Section 4.2.2.3) }\end{array}$ & \\
\hline $\begin{array}{l}\text { 3. Develop } \\
\text { the IS } \\
\text { Artefact }\end{array}$ & $\begin{array}{l}\text { Domain Ontology of BPC } \\
\text { - Ontology capture } \\
\circ \text { Analysed the knowledge } \\
\text { sources in detail } \\
\circ \text { Identified ontological } \\
\text { elements: concepts, } \\
\text { hierarchical relationships, and } \\
\text { decision-making relationships } \\
\text { - Knowledge organisation } \\
\circ \text { Synthesised the ontological } \\
\text { elements } \\
\circ \text { Organised the ontological } \\
\text { elements using a layered } \\
\text { structure }\end{array}$ & $\begin{array}{l}\text { - Lightweight ontology of BPC } \\
\text { (Figure 4.8) } \\
039 \text { salient concepts (Table } \\
4.6 \text { ) } \\
\text { - Five types of hierarchical } \\
\text { relationships } \\
\text { - Heavyweight ontology of } \\
\text { BPC } \\
\text { - Decision-making } \\
\text { relationships (Section } \\
4.3 .2 .3 \text { ), which turns the } \\
\text { lightweight into the } \\
\text { heavyweight ontology }\end{array}$ & $\begin{array}{l}\text { RQ2, } \\
\text { RQ3 }\end{array}$ \\
\hline
\end{tabular}




\begin{tabular}{|c|c|c|c|}
\hline & $\begin{array}{l}\text { Triangulation Evaluation } \\
\text { - Compared the BPC ontology } \\
\text { with a version generated by } \\
\text { OntoGen } \\
\circ \text { Took abstracts of the same } \\
\text { knowledge sources as input } \\
\circ \text { Used OntoGen to generate an } \\
\text { ontological version } \\
\text { Compared our ontology with } \\
\text { the generated version }\end{array}$ & $\begin{array}{l}\text { - High coverage of domain } \\
\text { concepts and relationships } \\
\text { - High clarity of the domain } \\
\text { semantics } \\
\text { - Our ontology provides clearer } \\
\text { meaning and capturing both } \\
\text { hierarchical and decision- } \\
\text { making relationships. }\end{array}$ & \\
\hline \multirow{3}{*}{$\begin{array}{l}\text { 4. Develop } \\
\text { the } \\
\text { Instantiated } \\
\text { Artefact }\end{array}$} & $\begin{array}{l}\text { Decision Tool } \\
\text { - Based on the ontology } \\
\text { - Developed two prototypes } \\
\text { o Used the first one for gathering } \\
\text { feedback } \\
\text { Developed the second } \\
\text { prototype based on the } \\
\text { feedback }\end{array}$ & $\begin{array}{l}\text { - A decision tool with two main } \\
\text { functions } \\
\text { O Supporting the decision to } \\
\text { crowdsource (Figure 4.16) } \\
\text { Supporting process design } \\
\text { (Figure 4.17) }\end{array}$ & \multirow{3}{*}{ RQ4 } \\
\hline & $\begin{array}{l}\text { Experimental Evaluation } \\
\text { - Conducted six experiment } \\
\text { sessions } \\
\text { - } 190 \text { participants } \\
\text { - Two experimental settings } \\
\circ \text { One group used the tool } \\
\circ \text { The other without the tool } \\
\text { (baseline) }\end{array}$ & $\begin{array}{l}\text { - Group using the tool shows } \\
\text { higher performance than the } \\
\text { baseline. } \\
\text { (p-value }=0.03 \text { for the } \\
\text { statistical difference in Tool } \\
\text { 1) } \\
\text { (p-value }<0.001 \text { for the } \\
\text { statistical difference in Tool } \\
\text { 2) }\end{array}$ & \\
\hline & $\begin{array}{l}\text { Focus Group Evaluation } \\
\text { - Conducted } 2 \text { focus groups } \\
\text { - } 10 \text { participants } \\
\circ 6 \text { crowdsourcing experts } \\
\circ 4 \text { PhD students with related } \\
\text { backgrounds }\end{array}$ & $\begin{array}{l}\text { - Strong evidence that the tool } \\
\text { provides structured } \\
\text { information } \\
\text { - Mixed evidence that the tool } \\
\text { frames and changes } \\
\text { participants' decisions } \\
\text { - Strong evidence of ease of use } \\
\text { - A few suggestions for } \\
\text { improvements of the tool }\end{array}$ & \\
\hline
\end{tabular}

Based on the research results summarised in Table 5.1, we now discuss the answers of each research question. 


\section{RQ1. What are the main building blocks of BPC that can be identified in the domain?}

We addressed this question by identifying 12 building blocks pertaining to BPC. They are captured in Table 4.2, and listed here: quality control, incentive mechanisms, crowd management, task design, task assignment, result aggregation, workflow design, capability and characteristics of crowdsourcing, circumstance to crowdsource and decision factors, platform, output and technical configuration. These building blocks were synthesised from the knowledge sources in the domain. Indeed, each of them was synthesised from and supported by more than ten knowledge sources. This strong support indicates that the identified building blocks are the most salient elements in the domain. The support further indicates that these building blocks are highly repeatable in crowdsourcing processes. A combination of salience and repeatability suggests that the identified building blocks are the key elements of BPC.

We further examine whether the identified building blocks are at the right abstract level to conceptualise BPC, which must balance between the high and low levels of granularity. In fact, they are at the right level. With high granularity, we have showed that these building blocks are abstract enough to conceptualise BPC. This can clearly be seen when the building blocks were utilised to develop a model conceptualising BPC and validated through the case study (Section 4.2). With low granularity, each building block can be further decomposed into processes, activities, data, and data entities, as shown in Figure 4.8. In other words, the building blocks do not just stay in the high abstraction, but link to detailed elements to describe and structure BPC. Consequently, we conclude that the 12 identified building blocks are at the right level to conceptualise BPC. Thereby, we answer RQ1.

\section{RQ2: What are the structures of the identified building blocks for conceptualising BPC?}

We addressed this question at three levels of abstraction. At a very high level, we propose a three-stage framework arranging the abstract activities of BPC. In the framework, BPC is structured as a sequence of three stages: decision to crowdsource, design, and configuration (Figure 2.3). The decision to crowdsource is the starting point of BPC, where organisations consider the appropriateness of the crowdsourcing approach. After 
the decision to crowdsource, design concerns what have to be made to instantiate a concrete crowdsourcing process. Then, configuration concerns the materialisation of a design into a concrete system. This abstract structure was proposed through the narrative review (Section 2.4), and confirmed by the scoping review where the three stages were used to organise the building blocks of BPC (Section 4.2).

At the conceptual level, we developed a process model that logically organises the activities necessary to be considered when establishing BPC. The model consists of seven components covering the main BPC activities (Figure 4.3). These activities represent the salient building blocks in the domain, which were identified by the scoping knowledge sources. The activities have been structured and put together based on both the timeline when the activities should be formed and their relationships with the three aforementioned abstract stages. As a result, the process model structures essential BPC activities and their relationships, which shows how to establish BPC.

To test the structure of the process model, we used the case study approach. More precisely, we examined the model in two existing crowdsourcing projects. The case study results suggest the high adequateness and usefulness of the model in structuring the main projects' activities. It can be observed from the results that the model lays a foundation of how organisations might rationally analyse and manage the crowdsourcing activities. Furthermore, the results give insights into how the model and its structure can be applied in practice. In summary, we address the research question at this conceptual level by structuring the identified building blocks into a validated BPC process model.

Finally, we also addressed this question at the ontological level. At this level, we propose a lightweight ontology organising the identified building blocks and their decomposed elements in a layered structure (Figure 4.8). In this structure, the identified building blocks form the inner layer of the ontology, and sequentially link to their decomposed elements in the other process, data, and data attribute layers. This shows the relationships between the identified building blocks and their decomposed elements. The details of the ontology will be discussed in the next section.

Overall, we answer RQ2 through a combination of the three-stage framework, process model, and lightweight ontology. Each of them provides a structure of the identified building blocks at different levels of abstraction. We note that within these levels of 
abstraction, the process model should account for the largest part of answering RQ2 since it directly structures the BPC building blocks.

We note that when addressing RQ1 and RQ2, we discover the important role of the decision to crowdsource in BPC establishment. This importance led us to an analysis of the decision to crowdsource and its decision factors. The results reveal nine factors and sixteen sub-factors that influence the decision to crowdsource (Table 4.3). We suggest that by focusing on these decision factors, organisations can evaluate whether crowdsourcing is appropriate for their contexts. The results also reveal the relationships between these factors, which allow us to structure the decision factors into a decision framework considering the task, people, management and environmental concerns. The decision framework suggests to managers what criterion need to be addressed before making the decision to crowdsource (Figure 4.4).

\section{RQ3: How to construct a domain ontology of BPC that organises the unstructured knowledge sources in the domain?}

This research question and the next one are the 'how to' research questions, typically formulated in design science (Gregor \& Hevner, 2013). This type of question requires us not only to describe the outcome artefacts but also to show the process generating and validating the artefacts. Thus, we organise our answers for the two research questions around three aspects: build activity, outcome artefacts, and evaluate activity.

We built a domain ontology of BPC through two main activities: ontology capture and knowledge organisation. The ontology capture analysed the knowledge sources for ontological elements. The results reveal 39 salient concepts, a large number of hierarchical relationships grouped in five categories, and more than 100 decision-making relationships (Section 4.3.2). Together, the ontological elements provide the materials for the ontology construction. Using the materials, the knowledge organisation selected the salient ontological elements, which were based on the 'wisdom of researchers'. In this activity, we organised the selected elements through a trial-and-error process with different structures. We finally decided to use a layered structure to organise the selected elements, which manage complexity by arranging them into layers (Figure 4.8). The result of the build activity is an ontology of BPC. 
We proposed a new ontology that provides a sound knowledge base supporting the establishment of BPC. Two versions of the ontology have been proposed: lightweight and heavyweight. The lightweight ontology organised the captured concepts and hierarchical relationships in the domain (Figure 4.8). It thus provides an ontological structure necessary to understand the constituents of BPC. Based on the lightweight one, the heavyweight ontology added decision-making relationships to the domain (Figure 4.9). Thus, it provides reasoning knowledge that guides organisations what should be done in a specific BPC activity. Taken together, the lightweight and then heavyweight ontology serve as a blueprint, supporting organisations in their BPC establishment.

The ontology was evaluated by triangulation. We compared our ontology with a version generated by OntoGen (Fortuna et al., 2007). The results are the high consistency with the main ontological elements, which suggests a high coverage of our ontology. Further, our ontology advances the automatic version in terms of clarity, when it captured both hierarchical and decision-making relationships and provided clearer meanings of the ontological concepts (Section 4.3.3). As a result, the triangulation increases the validity and confidence of the proposed ontology.

In summary, we propose a domain ontology of BPC that organises the knowledge in the domain. We also explicitly describe how the ontology was built and evaluated. Thereby, we answer RQ3.

\section{RQ4: How to construct a decision tool supporting organisations in establishing BPC?}

As this is again a 'how to' research question, we answer it around three aspects: build activity, outcome artefacts, and evaluate activity. This dissertation developed a decision tool supporting managers and process designers addressing the complexity of BPC. The tool was constructed based on the BPC ontology. The use of ontologies backing decision support systems is largely suggested in the literature (Delir Haghighi et al., 2013; Miah et al., 2014; Osterwalder, 2004; Ostrowski et al., 2014). We instantiated the tool through two prototypes. The first one was used for gathering feedback. The second one used the feedback for improving the tool functionality. This prototyping approach allows us to manage the rigour of the tool construction through iterations of prototyping and 
improvement (Kordon, 2002; Lim et al., 2008). The construction results are a decision tool supporting BPC establishment.

Regarding the outcome artefact, we provided a decision support tool for BPC establishment. The tool has consolidated and guided decision makers through a structured decision-making process. It consists of two main functions: the decision to crowdsource and process design. In supporting the decision to crowdsource, it enables decision makers to define their crowdsourcing context and analyse whether crowdsourcing is a suitable approach (Figure 4.16). In supporting the process design, the tool provides 'what-if' analysis and advice regarding project conditions and intervention plans (Figure 4.17). We note that there is a strong link between the tool and the BPC ontology, which served as a knowledge module of the tool (Figure 4.11). This link ensures the tool interoperability and its capability for supporting the entire BPC process.

For evaluating the utility of the constructed tool, we conducted several experiments and two focus groups. In the experiments, 190 participants tested the tool for supporting the decision to crowdsource and process design. The experimental results provide strong evidence for the positive impact of the tool. That is, the groups using the tool show higher performance than the other groups. This high higher performance is statistically confirmed in both the decision to crowdsource ( $\mathrm{p}$-value $=0.03$ as shown in Table 4.20) and process design ( $\mathrm{p}$-value $<0.001$ as shown in Table 4.21). Consequently, the experimental results allow us to confirm that using the tool can increase decision performance when establishing BPC.

We also evaluated the tool using the focus group approach. More precisely, we conducted two focus groups: one group of crowdsourcing experts and the other group of $\mathrm{PhD}$ students with backgrounds related to crowdsourcing. The findings of the focus groups confirm the tool utility for providing and structuring information on BPC establishment (Section 4.4.4). The utility template, like "it [the tool] is definitely promoting a lot of the right things to help make a correct decision", have been repeatedly found in the focus group discussion. Furthermore, the results show that the tool is easy to use. Yet, evidence on whether the tool can change participants' decisions on certain BPC decisions are mixed. Some participants after using the tool changed some of their decisions, while others did not. Some suggestions for improving the tool are also noted. 
Reflecting on the evaluations, the combination of the experimental and focus group results confirms the tool utility quantitatively and qualitatively. We highlight that the combination of the two evaluation techniques has strengthened the certainty of the confirmation. More precisely, the experiments results are based on statistical power. Complementarily, the focus group results are based on thematic templates. The use of multiple complementary evaluation methods has been repeatedly highlighted in the design science literature (Mettler et al., 2014; Sonnenberg \& vom Brocke, 2012a; Venable et al., 2016).

Overall, the combination of the tool development, its prototypes, the experimental and focus group evaluations that confirm the tool utility in supporting BPC establishment, has answered the final research question.

\subsection{Chapter Summary}

This chapter has viewed the research results from an integrated perspective. It presented four main integrated findings, highlighting the interrelated nature of the research process. The chapter also answered the research questions by thematically consolidating the research findings. As a result, we suggest that although the outcomes of the research can be seen in each of the research stages, they can also be grouped as thematic and integrated findings, which provide a comprehensive picture of the research outcomes.

The following chapter discusses the contributions and limitations of the research. 


\section{DISCUSSION}

This chapter discusses the research outcomes in light of its main contributions, implications and limitations. The chapter starts with four major contributions of the research. We then discuss how the research findings contribute to the knowledge base, in terms of design products and design processes. Following this is a discussion of contributions towards organisational practice. Then, limitations are discussed.

\subsection{Research Contributions}

Having been a design science endeavour, our work contributes knowledge throughout its research activities, from problem definition, to sound research process, to solutions and their reflection, and to communication of the research results. Consequently, as it is very hard to provide an exhaustive list of all research contributions, we have identified four major contributions. Each of them is discussed in the following sections.

\subsubsection{A New Approach for Establishing Crowdsourcing as an Organisational Business Process}

At the beginning of the dissertation, we noted that organisations face the challenge of how to establish crowdsourcing as an organisational business process. Despite a decade of research, most crowdsourcing research still relied heavily on an ad-hoc perspective, studying individual aspects of the crowdsourcing process. In many cases, these studies explored and investigated crowdsourcing as a one-off process, rather than a common organisational practice. Consequently, the challenge still remains.

Our first approach to this challenge is the introduction of a business process lens on crowdsourcing processes, designating the concept of BPC. While the term BPC was used previously (La Vecchia \& Cisternino, 2010), it is this dissertation that elevates and defines the BPC concept. We suggest that BPC is best conceptualised as balancing between the business process construct and the crowdsourcing construct. Consisting of the business process construct, BPC serves as a template for creating multiple, real life instances of the same crowdsourcing process. Our conceptualisation of BPC is partly theoretical, 
based on crowdsourcing literature and business process literature, and partly empirical, based on our observation that existing crowdsourcing processes have several activities that are repeatedly performed, as confirmed below.

The BPC conceptualisation can only stand with the condition that there are common repeatable activities of existing crowdsourcing processes. In this research, the condition has been met. Our scoping review has confirmed that there is a set of common activities in the existing crowdsourcing processes, repeatedly found in multiple knowledge sources. These common activities, which have also been supported by other recent reviews (Amrollahi, 2015; Hosseini et al., 2015a), enable us to confirm the condition backing the BPC concept. Further, these common activities also suggest the basic building blocks of BPC (Table 4.2).

Using the building blocks identified by the scoping review, we conceptualise BPC through a process model. The model, on the one hand, clarifies the BPC conceptualisation through a process viewpoint with multiple structured activities that are necessary to establish crowdsourcing as an organisational business process. On the other hand, the model keeps the BPC conceptualisation focus. That is, the model focuses on the core repeatable building blocks of BPC. With this focus, the model defines the abstract structure of BPC, and treats new crowdsourcing processes as real life instances of the same core building blocks (Figure 4.3). All in all, the process model, with its focus and business process lens, places BPC in a space quite distinct from one-off processes and their instances (Lüttgens et al., 2014; Stol \& Fitzgerald, 2014).

\subsubsection{The Importance of the Ontology}

Having introduced the concept of BPC, the dissertation also proposes an ontology that offers knowledge structures around this concept. The ontology provides various unique benefits in BPC conceptualisation. We now discuss these benefits from three main research perspectives of the dissertation: IS, design science, and DSS.

Ontologies have played an important role in representing IS domains (Fonseca \& Martin, 2007; Guo et al., 2009; Wand \& Weber, 1995). In this vein, our proposed ontology represents the BPC domain. More precisely, it defines BPC building blocks, processes, data, and data entities. It also structures the domain by presenting the hierarchical and decision-making relationships (Figure 4.8 and Figure 4.9). As a result, the ontology offers 
a scaffold for understanding the BPC domain. The representation of the ontology can be further characterised in two aspects: clarity (Akdemir et al., 2008; Fan et al., 2016) and coverage (Fan et al., 2016; Shanks et al., 2003).

The BPC ontology has high clarity contributing to the understanding of the domain, which can be seen via three points. First, it defines not only domain concepts but also hierarchical relationships and decision-making relationships, which increases shared understanding in the domain. Second, the ontology helps reduce semantic ambiguity. As noted previously, conflicting views and opinions exist in the domain, which leads to certain levels of semantic ambiguity. The ontology manages the conflicts through the 'wisdom of researchers', using the majority of knowledge sources as an indicator to address the conflicts. Finally, a combination of the ontology with the conceptual model and decision tool has provided three levels of abstraction for understanding the domain (Figure 5.2). All these points contribute to the high clarity of the ontology.

The BPC ontology also has a high coverage of domain concepts and relationships. This high coverage comes mainly from our grounded approach, which allows the ontological elements freely emerge. As a result, the ontological elements cover diverse aspects of the domain. We note however that in the grounding process, we made a decision that might reduce the coverage level of the ontology. That is, the decision to focus on the concepts supported by at least ten knowledge sources. Acknowledging the concern, we however have retained our decision since we have to balance the trade-off between coverage and complexity. Further, the evaluation of the ontology has lately shown that our decision is appropriate. More precisely, the comparison of our ontology with a version generated by OntoGen has shown that the BPC ontology broadly covers the domain. These results confirm the high coverage of the BPC ontology.

Before moving to the next perspective, we note here the nature of our ontology. If we follow Sharman et al. (2004) classifying ontologies as: top-level, domain, and application, our ontology should be seen as a domain ontology since we strictly focus on the BPC area. Furthermore, it should be treated as an informal ontology, rather than a formal one that would be defined using representation formalism languages. We nevertheless note that developing an informal ontology before transferring it into a formal one is a common, acceptable practice (Wong, Liu, \& Bennamoun, 2012). Considering the BPC ontology in 
the lightweight-heavyweight continuum (Corcho et al., 2003), our work is aligned to the heavyweight ontologies since we examine not only concepts but also decision-making relationships and business rules in the BPC domain. As a result, we have contributed a heavyweight informal ontology to the BPC domain.

Design science, as a significant paradigm of IS research, also highlights the role of ontologies. While agreeing with the ontology roles aforementioned in the IS perspective, design science in particular suggests the contributions of ontologies for building knowledge bases (Miah et al., 2007; Miah et al., 2014; Osterwalder \& Pigneur, 2004; Ostrowski et al., 2014). In the dissertation, the ontology has offered a BPC knowledge base. It builds the knowledge base through structuring the key concepts, hierarchical structures, and decision-making relationships, from which knowledge can be inferred. Furthermore, the knowledge base role of the ontology has been clearly revealed when the ontology formed the basis for tool construction. This is because founding artefact construction is a distinctive characteristic of knowledge bases (Hevner \& Chatterjee, 2010). We note that the knowledge base offered by the ontology should not be limited only to construct the decision tool, but can also be used to constructing other designscience artefacts, e.g. artefacts to standardise crowdsourcing processes. In short, we offer an ontological knowledge base for artefact development in the BPC domain.

Finally, we consider the ontology from the DSS (decision support system) perspective. In DSS literature, we identify two main roles of ontologies. The first role views ontologies as vocabulary frameworks defining terms, concepts and decision alternatives for certain DSS environments (Chen, Chen, Hsu, \& Li, 2011; Van Valkenhoef, Tervonen, Zwinkels, De Brock, \& Hillege, 2013). The second role, extending the first one, views ontologies as reasoning means, which structure logics of the DSS solutions (Amailef \& Lu, 2013; Gennari et al., 2003; Miah et al., 2007). The BPC ontology in the current study is aligned with the second role, ontology-supported reasoning, for three reasons. First, the ontology helps develop reasoning knowledge, which has been showed via the exemplar of the decision tables (Table 4.9, Table 4.10, and Table 4.11). Second, the reasoning role is aligned with the knowledge base role of the ontology, mentioned earlier in the design science perspective. Lastly, the ontology was actually integrated into the decision tool as a reasoning module (Figure 4.11), which confirms its role as the ontology-supported reasoning means. 
Overall, the BPC ontology plays several critical roles in the current research. It is a domain ontology clarifying and covering the BPC domain. It also serves as a designscience knowledge base consolidating the existing knowledge for artefact development. Furthermore, it as a heavyweight ontology supports reasoning, which has been operationalised in the decision tool. These roles suggest the value of the BPC ontology.

\subsubsection{Empirical Findings}

In the above discussion, we have discussed BPC conceptualisation and its ontological structure for establishing crowdsourcing processes. In addition to these theoretical efforts, the dissertation also brings empirical results that provide evidence on how our theoretical work can be operationalised to improve the establishment of BPC. Empirically, we constructed a decision tool and assessed it using experiments and focus groups.

The successful construction of the tool means four things. First, the tool construction has proved that BPC can actually be operationalised in practice. Second, the tool, which was developed based on the BPC ontology, has demonstrated the feasibility of the ontology. That is, the ontology can be implemented in a working system. Third, the construction has created an instantiation artefact (Hevner et al., 2004; March \& Smith, 1995), which is a decision tool providing a means for decision makers to establish BPC step-by-step and to guide them in this establishment. Finally, the tool enables concrete assessments of its utility towards BPC establishment.

A mixed method was used for empirical assessment of the tool. We deployed a sequence of 1) the experiments to test whether the tool is useful for improving performance on BPC establishment and 2) the focus group to understand what aspects of the tool's usefulness are perceived by the participants. In the experiments consisting of 190 participants, the findings confirm that the use of the tool leads to better performance on both functions of the tool: the decision to crowdsource and crowdsourcing process design. From the results, we suggest that the tool is useful for BPC establishment. We note that although both functions are useful, and both are statistically supported, the support for process design ( $\mathrm{p}$-value $<0.001)$ is stronger than for the decision to crowdsource ( $\mathrm{p}$-value $=0.03$ ). The experimental results alone cannot explain the difference, which has addressed in the focus group evaluation. In summary, the experiments provide empirical evidence suggesting 
the usefulness of the tool. While this usefulness is supported statistically, some of its aspects should be further evaluated and discussed.

Serving our intention to further evaluate the tool, two focus groups were conducted to gain insights on what aspects of the tool utility were perceived by the participants. The focus group results show that the tool benefits in terms of structuring BPC establishment and providing additional information for making informed decisions. It is also found that participants when using the tool have a positive perception towards ease of use, and they suggest a few possible improvements. There are mixed results on whether the tool may change the participants' decisions. Overall, the focus group results are positive towards the tool utility. They also help as a support to compare with the experimental results, as presented below.

Together, the two evaluation results enable us to confirm the tool utility, using both quantitative, individual-based, and controlled experiments, as well as qualitative, groupbased, and likely naturalistic focus groups. It is also interesting to discuss their complementary findings. The focus group findings suggest that the tool is more useful for providing additional information than for changing participants' decisions. This provides a possible explanation for the different levels of support for the tool's utility in the experimental results regarding the decision to crowdsource and process design. Possibly, the equal support regarding the decision to crowdsource comes from the moderate ability of the tool that might or might not change participants' decisions, while the strong support regarding the process design comes from the strength of the tool that provides additional structured information in the design process.

Overall, the importance of the dissertation relies not only on theoretical efforts, but also on having as much empirical evidence as possible. We have discussed the evidence from the experiments with 190 participants, and two focus groups. Apart from these, other empirical evidence was also collected and incorporated into the research results, including case studies of two crowdsourcing projects, and a pilot experiment with 46 participants. As a result, the empirical results have complemented and supported our theoretical efforts on BPC establishment. 


\subsubsection{Progression of Business Process Crowdsourcing}

So far, we have presented our theoretical and empirical contributions to BPC establishment, which are expected to move the development of the BPC concept forward. To clarify this movement, we examine the progression of the concept in comparison with the literature review in Chapter 2. In that chapter, we reviewed four main research strands: the broad concept of crowdsourcing, crowdsourcing classifications, crowdsourcing processes, and the research foci of BPC. At that time, the four review strands covered quite broad aspects of crowdsourcing to form a foundation for our research. It is instructive if we re-examine these strands, focusing only on the concept of BPC.

Focusing on the BPC concept, we propose five phases of the concept progression. These phases are shown in Figure 6.1. In the first phase, research conceptualised the overarching crowdsourcing concept by specifying its ideas and definitions (Estellés-Arolas \& González-Ladrón-de-Guevara, 2012; Howe, 2006a), but did not mention BPC. The second phase started to classify different elements (Schenk \& Guittard, 2011; Zhao \& Zhu, 2014), in order to structure the crowdsourcing domain. At first, these structures were simple, just focusing on particular crowdsourcing elements. Also in this phase, a large number of studies researched ad hoc foci of crowdsourcing, which created a 'shopping list' of individual elements. Only in the third phase, the high-level building blocks of crowdsourcing processes became available. A few researchers were able to combine the individual elements forming an abstract crowdsourcing process and its building blocks (Amrollahi, 2015; Pedersen et al., 2013; Zogaj et al., 2014). Some of these building blocks are abstract and repeatable, which can be synthesised into BPC building blocks.

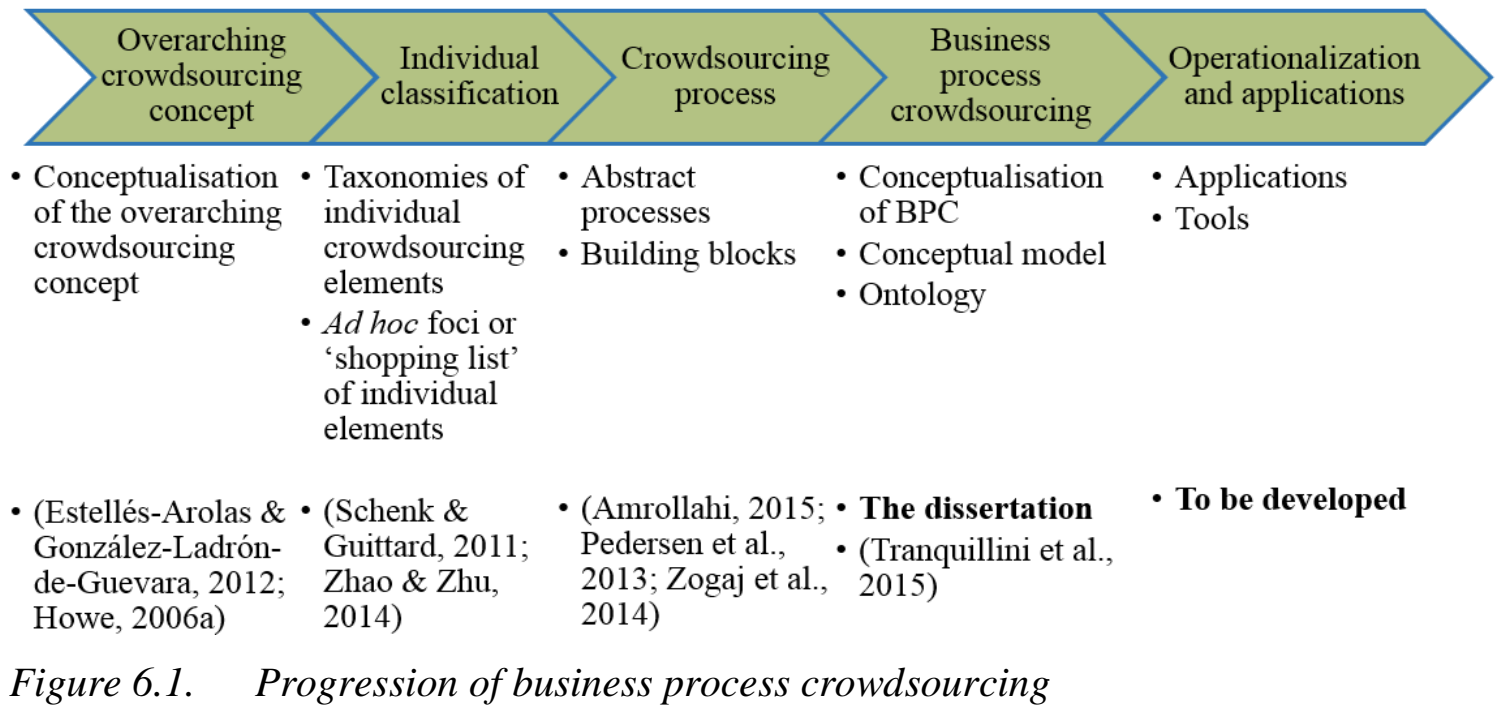


The fourth phase is the ongoing position of BPC. The target of this phase is to conceptualise and model the BPC concept leading to the proposition of reference models and ontologies. This phase is the focus of the dissertation. The dissertation conceptualised BPC using the building blocks synthesised from the scoping review (Section 4.1). We developed a process model (Section 4.2) and a heavyweight ontology guiding BPC (Section 4.3), which together provide a solid knowledge base for BPC establishment. Apart from our work, this phase also includes other recent models (Hetmank, 2014; Tranquillini et al., 2015), which enact and implement business processes based on crowdsourcing. Collectively, since this phase consists of our work that provides means to conceptualise, analyse, and design BPC, and the other work that provides means to enact and implement BPC (Hetmank, 2014; Tranquillini et al., 2015), this phase offers a solid scaffold supporting the whole business process based on crowdsourcing, from analysis, to design, and to implementation.

In the last phase, the models and ontologies of the previous phase can be applied to IS applications. Although our decision tool is an example of such applications (Section 4.4), we suggest that this is a to-be-developed area where diverse BPC tools and applications should be developed.

Overall, these five phases reflect the progression and expected development of BPC. They show the evolution of the domain, from an overarching concept, to individual structures, to abstract processes, to business process crowdsourcing, and to diverse BPC applications. Through this evolution of BPC, we think that the domain will continue progressing and further providing more applications to benefit organisations.

In summary, this section showed our contributions to the BPC domain. The contributions include the introduction of BPC conceptualisation, important roles of the ontology, empirical findings that show how our work operationalise and supports BPC, and progression of BPC. Together, they allow us to suggest that our work has contributed to move BPC forward in its progression in order to actually become an organisational business process. 


\subsection{Other Contributions to Design-Science Knowledge Base}

Apart from the four major contributions discussed above, the research also yields other contributions to the knowledge base, which is a key requirement of design science research. As design science research involves two complementary aspects: design product and design process (Gregor \& Jones, 2007; Hevner et al., 2004), we structure the research contributions around both of them.

\subsubsection{Design Product - Three Outcome Artefacts}

From the design product viewpoint, the central contributions of this research are purposeful artefacts for conceptualising, structuring and supporting BPC. More precisely, three artefacts are added to the BPC domain: a process model, an ontology, and a decision tool. These artefacts have been extensively discussed in this dissertation and originally presented as Figure 5.2. We now clarify their contributions by adapting the contribution framework proposed by Gregor and Hevner (2013). Figure 6.2 presents this adaption, which has included the three artefacts in relation to their contribution types.

\begin{tabular}{|c|c|c|}
\hline & Outcome Artefacts & $\begin{array}{c}\text { Contribution Types } \\
\text { (Gregor \& Hevner, 2013) }\end{array}$ \\
\hline \multirow{3}{*}{ 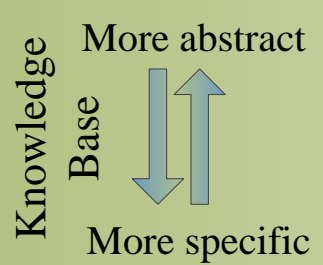 } & Process model & Conceptual model \\
\hline & Ontology & Model \& Operational rules \\
\hline & Decision tool & Instantiation \\
\hline
\end{tabular}

Figure 6.2. Research design products (adapted from Gregor \& Hevner, 2013)

In Figure 6.2, the outcome artefacts are positioned in three levels of abstraction. In the most abstract level, the research contributes a new BPC process model. Although a few models and frameworks have already been proposed in the crowdsourcing field, our artefact is the first to conceptualise BPC. More precisely, the model creates a new basis for conceptualising BPC by combining its building blocks identified in the domain (Figure 4.3). We also demonstrate how to use the model in practice, via case studies. As a result, the artefact fulfils the current need for a model guiding organisations establishing a business process based on crowdsourcing (Amrollahi, 2015; Djelassi \& Decoopman, 2013; Lüttgens et al., 2014). 
Moving to the second level of abstraction, the dissertation offers a BPC ontology. The roles of the ontology have been extensively discussed in Section 6.1.2. Here we further highlight that the ontology also serves as an IS artefact per se (Osterwalder, 2004). The ontology addresses the lack of a heavyweight ontology in the domain. Although two prior lightweight ontologies were proposed (Hetmank, 2014; Luz, Silva, \& Novais, 2015), they mainly attempt to classify and define crowdsourcing concepts. Thus, the domain has still needed an ontology to structure these concepts and manage domain reasoning. Our ontology addresses this need and extends the existing work by not only defining concepts, but also 1) capturing hierarchical relationships that classify the concepts into main building blocks, processes, data, and data attributes, and 2) adding decision-making relationships that enhance reasoning knowledge. In short, we contribute the ontology as an artefact addressing the important need in the BPC domain.

In the specific level of abstraction, we built and validated a decision support tool for establishing BPC, which according to Gregor and Hevner (2013) is an instantiated artefact. As an instantiated artefact, the tool operationalises the ontological structures in the computer-based prototypes. Furthermore, it offers two further contributions to crowdsourcing DSS development. First, although a few DSSs exist in the crowdsourcing field, many of them aim at supporting individual workflows like task assignment (Geiger $\&$ Schader, 2014) and result aggregation (Prokesch \& Wohlenberg, 2014) rather than the whole integrated crowdsourcing process. Our tool extends these DSS works when it provides support for the whole BPC. Second, the domain had noted a shortage of empirical evidence on the use of DSS systems, e.g. in the case of crowdsourcing recommendation systems that "one of the most fundamental issues for future research [...] is the empirical evaluation of its general utility to the crowdsourcing process" (Geiger \& Schader, 2014, p. 11). We have carefully evaluated the tool using both experiments and focus groups. The empirical results from the tool evaluations address the above shortage, and show the empirical impact of DSSs on supporting crowdsourcing.

To sum up, from the design product viewpoint, the process model, ontology and decision tool are the innovative artefacts that we contribute to the BPC domain. We note that these artefacts can be used either as separate artefacts, or as a set of artefacts in capturing BPC knowledge from abstract to concrete as illustrated in Figure 6.2. Whether separately or as a set, their use guides organisations in their BPC establishment. 


\subsubsection{Design Process - A Design Method for Emerging DSS Environments}

As we have elevated the design process viewpoint to be as equally important as the design product viewpoint, the dissertation established its research process carefully. Reflecting this process, we highlight its systematic and transparent characteristics. The systematic characteristic is reflected through our justification of every research technique used in the process, and in particular, through the choice of a scoping review that refers to a systematic procedure of analysing and synthesising a body of knowledge (Paré et al., 2015). The transparent characteristic is reflected through Chapter 3 and Chapter 4, where we described the research activities and research decisions as explicitly as possible.

Given the systematic and transparent characteristics, our research process, to some extent, generalises a design method that guides DSS development in an emerging areas. We discussed the proposed method in our recent publication (Thuan, Antunes, \& Johnstone, 2015a). The following section summarises the key points of the method, focusing on the need for such a design science method, our proposed method, and its evaluation.

The need of design science methods for emerging DSS areas. There is a high demand for design science methods addressing emergent DSS areas, like big data, social media, and mobile computing. We have discussed this demand for emerging areas in Section 3.2, and further specify it here for DSSs in particular. To develop decision support systems in these emerging areas, the current dissertation, consistent with recent DSS literature reviews (Arnott \& Pervan, 2008, 2014), suggests the key role of design science for two reasons. First, design science emphasises a rigorous approach to advance current knowledge on design and development (Hevner et al., 2004). This is highly applicable to emerging DSS environments, where decision-support tasks involve consolidating domain knowledge for better decision-making (Nemati, Steiger, Iyer, \& Herschel, 2002). Second, design science aims at developing innovative artefacts to address unstructured issues, which are also the major target of DSS research in emerging areas. As a result, design science is an appropriate paradigm to address the emerging DSS environments.

In spite of this appropriateness, the application of existing design methods may not be suitable for emerging DSS environments, where significant wickedness and dynamism exist. Most existing methods require either established theories or meta-artefacts related to the problem (e.g. Carlsson et al., 2011; Pries-Heje \& Baskerville, 2008). However, this 
requirement is hardly met in emerging areas, as explained by Paré et al. (2015, p. 188), that in such emerging issues/areas "an accumulated body of research exists but there is a lack of appropriate theories or current theories are inadequate in addressing existing research problems". Furthermore, the existing methods do not address the issue of lacking common understanding due to the unestablished nature of the emerging areas. Without a common understanding about related concepts, there is a high risk of providing irrelevant and/or incomplete support for decision makers. Given this situation, we highlight the need of a design method specifically tailored to emerging low-theoretical DSS environments.

A Design Science Method for Emerging DSS Environments. Addressing the need, we have proposed a design method guiding DSS development in low-theoretical domains. Since the method targets low-maturity domains, it should focus on alternative knowledge sources rather than theories. Again, we refer to the three types of knowledge sources suggested by Hevner and Chatterjee (2010): scientific theories; experience and expertise; and meta-artefacts. In the emerging and dynamic DSS environments, we suggest basing the method on the second type of knowledge source, leading to a design science method grounding experience and expertise reported in individual knowledge sources.

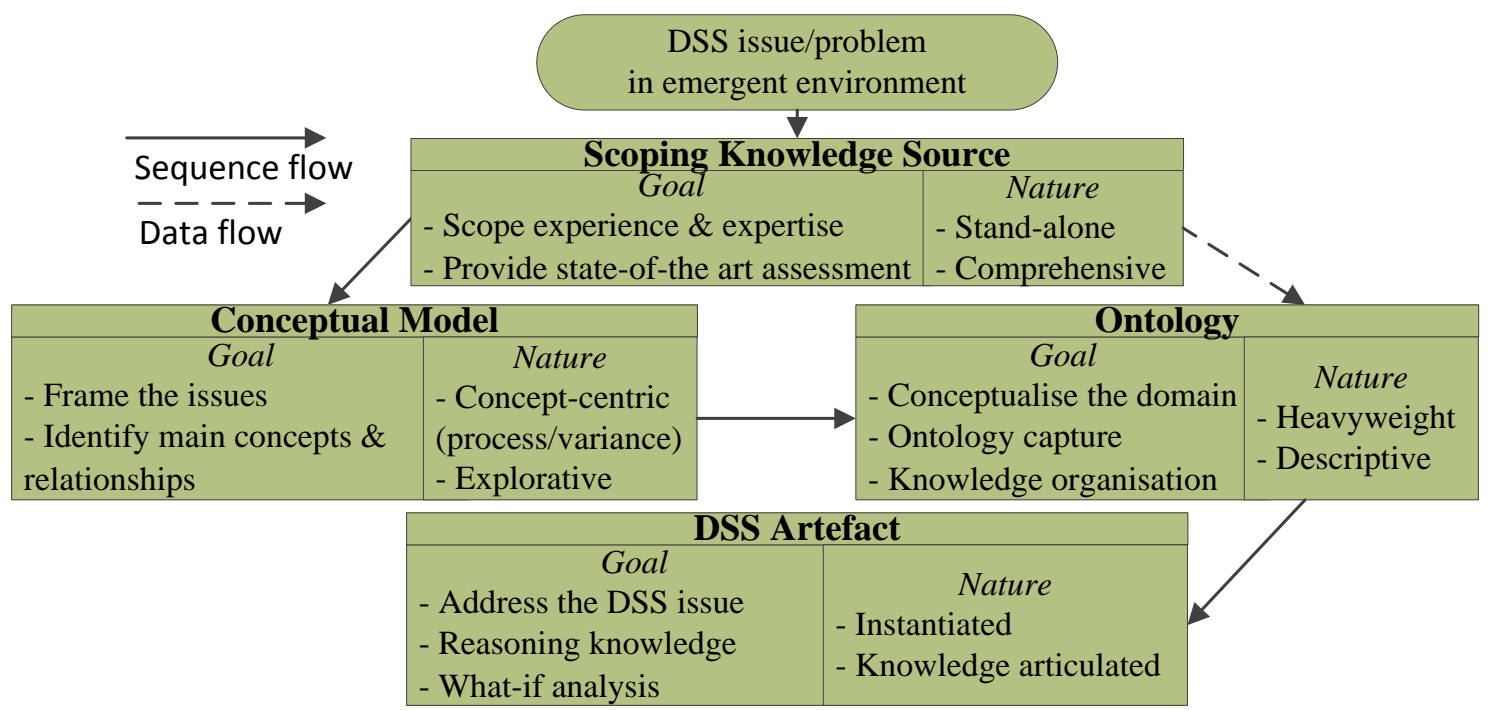

Figure 6.3. The SCOA method (Thuan et al., 2015a)

We named the proposed method as SCOA. SCOA defines four conceptual components: Scoping knowledge source, Conceptual model, Ontology, and DSS Artefact. Figure 6.3 graphically represent these components. Although they have already been used in design science research, SCOA represents them in a heuristic way that can ground experience and expertise knowledge in the low-theoretical DSS domains. To keep the discussion 
chapter focused, we do not present the details of the SCOA elements, but refer interested readers to our published paper (Thuan et al., 2015a).

Evaluation of SCOA. SCOA is evaluated by applying it in the context of a published project and illustrated in the context of the dissertation. We retroactively exercised SCOA with the published project focusing on business modelling, which was an emerging research area at that time and thus had no overarching theory (Osterwalder \& Pigneur, 2002). Similar to the SCOA method, the study conducted a review of the business model literature and used it as the main data for developing a conceptual model and an ontology. Using the outcome of the review, these authors elaborated a conceptual model, which was named 'the 4 pillars of the business model ontology' (Osterwalder \& Pigneur, 2002). Then, Osterwalder (2004) developed an ontology detailing the conceptual model, which was also influenced by the literature review (the data flow of the SCOA method). In particular, the author elected nine concepts that were "mentioned by at least two authors [references]" as the main concepts of the proposed ontology (Osterwalder, 2004, p. 11). Regarding artefact instantiation, a set of prototypes and an e-business model tool were developed (Osterwalder, 2004), which were based on the developed ontology.

In this published research project, the research process is very similar to the SCOA method, from reviewing the knowledge source, to the conceptual model, to ontology, and to DSS artefacts. Both the conceptual model and ontology are based on data extracted from the review, while together they support the artefact development. Furthermore, the idea of using concepts suggested by two references is what we referred to as the "wisdom of the researchers'. Besides the major similarity, some minor differences between the SCOA method and the case can still be found. For instance, the case adopts a narrative review, whereas SCOA suggests scoping knowledge sources, which is more rigorous (Paré et al., 2015) and thus more suitable for design science.

We have also demonstrated the proposed method in the context of the dissertation. More precisely, since BPC is a low-theory emerging area, the research process of the dissertation is strictly similar to SCOA. As shown in Chapter 4, we conducted the scoping review, constructed the process model and heavyweight ontology, and developed the decision tool supporting BPC establishment. As a result, SCOA has been successfully operationalised in the context of the dissertation. 
Table 6.1 summarises the evaluation and demonstration of the SCOA method. In Table 6.1, SCOA is largely aligned with several parts of the published project and are operationalised successfully in the dissertation. We note that using illustrative cases is one of the most popular ways to evaluate and demonstrate design method artefacts like SCOA (Peffers et al., 2012; Peffers et al., 2007). Given that, and that the SCOA method has been published (Thuan et al., 2015a), we suggest SCOA for guiding DSS development in areas where expertise and experience are dominant sources of knowledge.

Table 6.1. Summary of the two research projects using the SCOA language

\begin{tabular}{|l|l|}
\hline \multicolumn{1}{|c|}{ The business model } & \multicolumn{1}{c|}{ The current research } \\
\hline $\begin{array}{l}\text { Narrative review: identify the research gap and } \\
\text { explore the business model literature }\end{array}$ & $\begin{array}{l}\text { Scoping review: identify and synthesise main } \\
\text { building blocks of BPC }\end{array}$ \\
\hline $\begin{array}{l}\text { Explorative model: constitute the essential } \\
\text { business-model concepts }\end{array}$ & $\begin{array}{l}\text { Process model: } \text { construct a conceptual } \\
\text { process of BPC }\end{array}$ \\
\hline $\begin{array}{l}\text { Formal heavyweight ontology: provide a } \\
\text { foundation for understanding and measuring } \\
\text { business models }\end{array}$ & $\begin{array}{l}\text { Heavyweight } \text { ontology: } \text { structure domain } \\
\text { knowledge and capture business rules }\end{array}$ \\
\hline $\begin{array}{l}\text { Prototyping installation: verify the ontology } \\
\text { and provide a business modelling tool }\end{array}$ & $\begin{array}{l}\text { DSS artefact: design a decision tool } \\
\text { supporting BPC establishment }\end{array}$ \\
\hline
\end{tabular}

To sum up, we highlight the systematic and explicit characteristics of the dissertation's research process. Reflecting the research process, we propose the SCOA method for guiding emerging DSS environments, where theory is scarce and expertise and experience is dominant. We note that SCOA is the first effort to develop a design science method addressing emerging DSS domains.

\subsection{Contributions to Practice}

From a practical point of view, our study provides several practical contributions for organisations, decision makers, process designers, and project managers. The study provides organisations practical insights how to establish business processes based on crowdsourcing. In particular, organisations can use the conceptual model (Figure 4.3) and ontology (Figure 4.8 and Figure 4.9) as a blueprint for analysing, planning and deploying crowdsourcing processes. The model provides defined steps on how to establish a crowdsourcing process; and the ontology presents structured activities, data, and data 
attributes in order to accomplish these steps. Together, they enable organisations to take advantage by integrating crowdsourcing into their organisational business processes.

Another practical contribution comes from the proposed decision framework (Figure 4.4) and the set of decision tables (Table 4.9 Table 4.10 and Table 4.11). They support decision makers to evaluate whether crowdsourcing is an appropriate strategy. The framework guides decision makers on what factors should be considered when making crowdsourcing decisions. Based on the framework, the decision tables formulate decision rules, which interpret and ease the decision-making process (Huysmans et al., 2011). As a result, we suggest that organisations can use the decision framework and decision tables as a practical means to measure their readiness for crowdsourcing.

The study provides a computer-based tool supporting BPC establishment. The tool structures concepts, relationships, business rules, and what-if scenarios, which supports managers and process designers in their BPC decision. This practical support is highlighted in particular through the experiments, where the results show that the tool can improve decision makers' performance in both the decision to crowdsource and process design. Furthermore, while the tool supports are mostly important to process designers, they may also be relevant to crowdsourcing platforms. By examining the tool, platform developers can integrate similar supports to assist their crowdsourcing customers.

Finally, one interesting implication for the use of the tool comes from the focus group results, which show that the tool can remind users of certain crowdsourcing aspects that they forgot. This implies that the tool can be used for cross checking crowdsourcing projects. In particular, the tool can advise project managers what aspects that should be focused and what are possibly missing in their projects. Managers can also compare their project plan with what have been suggested by the tool in order to analyse and monitor the projects. This use of cross checking is further highlighted as the tool has been launched as a web tool, ready for managers to visit and exercise their crowdsourcing projects.

\subsection{Limitations of the Research}

Through a critical lens, this dissertation inevitably still has certain limitations. First, we understand the risk of building a knowledge base from very diverse knowledge sources, 
whose bias and limitations may be transferred to the knowledge base (Kitchenham, 2007). Understanding this concern, we however note that the use of diverse knowledge sources benefits from the 'wisdom of researchers', which utilises diverse opinions for developing a more comprehensive view of particular phenomena like BPC (Surowiecki, 2004).

Another limitation comes from our decision to choose the cut-off value of ten knowledge sources when applying the 'wisdom of researchers'. This decision might exclude some interesting concepts and relationships in the 'long tail' that were supported by less than ten sources. We nevertheless note that this decision was made in order to balance between complexity and representation. If the chosen value was low, many concepts would be selected and the complexity would increase (Jonker \& Pennink, 2010). In contrast, if the value was high, only a few building blocks would be selected, which would reduce the representation of the model and the ontology. After testing several values, we finally chose ten as the cut-off value that balances complexity and representation.

There is another limitation related to the development of the decision tool, which focuses on "proof of concept" prototypes. The tool was developed through the rapid prototyping method, and thus targeted only at the level of evaluation and demonstration. Although the tool can be redesigned to meet industry targets, future research could implement the tool by applying proper software engineering methods. Besides, when we experimented with the tool, it was recognised that using students as proxies for crowdsourcing decision makers would be a limitation. Yet, we note that the use of students to experiment with software tools is an acceptable practice. Sjøberg et al. (2005) survey 113 software controlled experiments and show that "87 percent of the subjects were students" (p. 751). Furthermore, we have addressed the limitation with complementary data, where we used focus groups with crowdsourcing experts in order to triangulate our results.

Finally, we have proposed the SCOA method in Section 6.2.2. Although the method has been demonstrated and evaluated by applying it to a published research project and our dissertation, this application is limited to two single cases. Consequently, to increase generalizability of the method, we would need to apply SCOA across a variety of DSS domains, where expertise and experience are predominant. 


\section{CONCLUSION AND FUTURE WORK}

In this thesis we have presented our efforts towards establishing crowdsourcing as an organisational business process, particularly in establishing Business Process Crowdsourcing, BPC. Returning to our starting point, we have observed that organisations are often unsure about the way to best structure different crowdsourcing activities and integrate them with other organisational business processes. It also seems that this challenge comes from the predominant view in the domain that crowdsourcing is a one-off process. Furthermore, promising research stream from business, such as the use of a business process lens, has scarcely been adopted in the domain.

Addressing the challenge, one main contribution of the research is the introduction of BPC that views crowdsourcing as an integrated business process, rather than a one-off process. We have established BPC from the design-centric approach in that the majority of our work is centred on the iterations of design and evaluation. These iterations bring to the domain several IS artefacts, starting with a knowledge base constructed from scoping knowledge sources. Based on the knowledge base, we propose and validate a process model guiding organisations to manage the main building blocks necessary to establish BPC. Building on the process model, we propose an ontology that structures the BPC domain. It consists of the concepts, hierarchical relationships, and decision-making relationships necessary to establish crowdsourcing as an organisational business process. We note that both the process model and ontology are founded on the knowledge base. Thus, they represent a synthesis of the domain knowledge and as a result add a step forward to the conceptual and ontological structure of the BPC domain.

As a benefit of the ontological approach, it enables us to implement a tool that assists managers and process designers addressing the complexity of BPC establishment. The tool helps make informed decisions in BPC establishment, including decisions in adopting, designing and configuring novel crowdsourcing business processes. Regarding its evaluation, the tool was assessed through experiments and focus groups, which have shown positive results towards the utility. These results, together with other evaluations throughout the research, suggest that the decision tool together with the conceptual model and BPC ontology should be utilised to establish BPC. 
Overall, our conclusions from this dissertation are positive towards the establishment of crowdsourcing as an organisational business process. The conceptual model, ontology, and decision tool, constructed and validated in the research, should be used to support the establishment. Consequently, we offer a body of knowledge, which has a strong theoretical ontological basis and promising empirical results, as a first attempt to establish the chosen phenomenon. By doing so, we hope that our attempt will motivate future researchers to investigate this important $\mathrm{BPC}$ domain. In this hope, we outline below a number of possible paths for future research.

\subsection{Future Work}

This dissertation opens several paths for further exploring the potential of BPC and analogous to the general research field of crowdsourcing. Future research should use the ontological elements: the concepts, hierarchical relationships, and decision-making relationships to design crowdsourcing experiments and field studies. In other words, the ontology serves as a basic for developing a broad research agenda in the area. In this agenda, additional research should focus on the decision-making relationships, given the low number of supporting sources for this type of relationships in the domain.

Future research should aim to move the knowledge provided by the artefacts built in this study forward to a higher level of abstraction with BPC design theories. This research direction, aligning with Gregor and Hevner (2013), suggests that with the proposed artefacts and instantiations, related design theories can be developed. Such BPC design theories can add generality to our proposed artefacts. For instance, as the BPC process model has been grounded from knowledge sources in the domain, the model is expected to be applicable in a variety of BPC contexts. Therefore, future research should further apply the model in different contexts, which will show its application principles and thus provide a basis for a theory explaining and predicting its use.

Our work also presents large research opportunities for further design-based efforts in both academia and industry. In particular, as a solid knowledge base in the domain, the BPC ontology can be used to construct different artefacts. Some that we can think of at this point in time are knowledge-based and collaborative-based systems, which are some 
common applications based on ontologies (Chandrasekaran, Josephson, \& Benjamins, 1999). Others, that only time could uncover, may emerge from the combination of interoperability, reasoning and knowledge organisation provided by the ontology.

From a technical perspective, while we have already proposed a decision tool for BPC, our work mainly focuses on the business process aspects of the crowdsourcing process. Thus, it is interesting to further develop and integrate our work from a more technical standpoint. We note that several toolkits that configure and program crowdsourcing processes have existed (Kittur et al., 2011; Kucherbaev et al., 2013; Little et al., 2010; Tranquillini et al., 2015). Given that, future research may investigate how to connect the decision-support focus in our work and existing technical toolkits. This connection would offer a decision support system that would assist organisations, from the time they analyse, model, and design BPC, until the time they instantiate it using a particular set of programming toolkits.

Lastly, we have proposed the SCOA method for guiding DSS development in emerging areas. Reflecting this proposal, we suggest two research directions. First, we acknowledge that while the method was evaluated in two projects, the concern over generalizability of the method is certainly a valid argument. Thus, an interesting research direction is to apply and test the method in multiple DSS low-theory areas, which would help strengthen method generalizability. Second, we note that DSSs may be developed in areas where a strong theoretical foundation may not be established yet. Consequently, we strongly call for new design methods and/or DSS methods to guide the development of decision support systems in the emerging areas.

In conclusion of this dissertation, it is clear that crowdsourcing has been an important sourcing strategy for organisations in the last decade, and this trend is expected to continue with business process crowdsourcing. By establishing crowdsourcing as an organisational business process, organisations can take full advantage of the strategy. This dissertation proposes a set of BPC artefacts that supports the establishment of crowdsourcing as an organisational business process. Furthermore, a solid knowledge base of BPC is built and enriched through theoretical, ontological, and empirical scaffoldings. This solid knowledge base is promising for the future development of the domain to progress towards a mature crowdsourcing strategy. 


\section{REFERENCES}

Adepetu, A., Ahmed, K. A., Al Abd, Y., Al Zaabi, A., \& Svetinovic, D. (2012). CrowdREquire: A Requirements Engineering Crowdsourcing Platform. 2012 AAAI Spring Symposium Series.

Afuah, A., \& Tucci, C. L. (2012). Crowdsourcing as a solution to distant search. Academy of Management Review, 37(3), 355-375.

Ågerfalk, P. J., \& Fitzgerald, B. (2008). Outsourcing to an Unknown Workforce: Exploring Opensurcing as a Global Sourcing Strategy. MIS Quarterly, 385-409.

Ågerfalk, P. J., Fitzgerald, B., \& Stol, K.-J. (2015). Crowdsourcing Software Sourcing in the Age of Open (pp. 45-60): Springer International Publishing.

Akdemir, U., Turaga, P., \& Chellappa, R. (2008). An ontology based approach for activity recognition from video. Proceedings of the 16th ACM international conference on Multimedia, 709-712.

Allahbakhsh, M., Benatallah, B., Ignjatovic, A., Motahari-Nezhad, H. R., Bertino, E., \& Dustdar, S. (2013). Quality control in crowdsourcing systems: Issues and directions. Internet Computing, IEEE, 17(2), 76-81.

Allahbakhsh, M., Ignjatovic, A., Benatallah, B., Beheshti, S., Bertino, E., \& Foo, N. (2012). Reputation management in crowdsourcing systems. Paper presented at the 8th International Conference on Collaborative Computing: Networking, Applications and Worksharing (CollaborateCom).

Alonso, O. (2013). Implementing crowdsourcing-based relevance experimentation: an industrial perspective. Information retrieval, 16(2), 101-120.

Alonso, O., \& Baeza-Yates, R. (2011). Design and implementation of relevance assessments using crowdsourcing. In P. Clough, C. Foley, C. Gurrin, G. F. Jones, W. Kraaij, H. Lee, \& V. Mudoch (Eds.), LNCS (Vol. 6611, pp. 153-164): Springer Berlin Heidelberg.

Amailef, K., \& Lu, J. (2013). Ontology-supported case-based reasoning approach for intelligent $\mathrm{m}$-Government emergency response services. Decision Support Systems, 55(1), 79-97.

Amrollahi, A. (2015). A Process Model for Crowdsourcing: Insights from the Literature on Implementation. Proceedings of the Australasian Conference on Information Systems 2015, Paper 18.

Anderson, D. R., Sweeney, D. J., \& Williams, T. A. (2011). Statistics for business and economics: South-Westerb, Cengage Learning. 
Andriole, S. J. (2010). Business impact of Web 2.0 technologies. Communications of the ACM, 53(12), 67-79.

Antunes, P., Herskovic, V., Ochoa, S. F., \& Pino, J. A. (2012). Structuring dimensions for collaborative systems evaluation. ACM Computing Surveys (CSUR), 44(2), Article 8.

Antunes, P., Zurita, G., Baloian, N., \& Sapateiro, C. (2014). Integrating decision-making support in geocollaboration tools. Group Decision and Negotiation, 23(2), 211233.

Archak, N. (2010). Money, glory and cheap talk: analyzing strategic behavior of contestants in simultaneous crowdsourcing contests on TopCoder.com. Proceedings of the 19th international conference on World wide web, 21-30.

Arnott, D., \& Pervan, G. (2008). Eight key issues for the decision support systems discipline. Decision Support Systems, 44(3), 657-672.

Arnott, D., \& Pervan, G. (2014). A critical analysis of decision support systems research revisited: the rise of design science. Journal of Information Technology, 29(4), 269-293.

Baba, Y., \& Kashima, H. (2013). Statistical quality estimation for general crowdsourcing tasks. Proceedings of the 19th ACM SIGKDD international conference on Knowledge discovery and data mining, 554-562.

Bacon, C. J., \& Fitzgerald, B. (2001). A systemic framework for the field of information systems. ACM SIGMIS Database, 32(2), 46-67.

Bailey, K. D. (1994). Typologies and taxonomies: an introduction to classification techniques: Sage.

Barclay, C., \& Osei-Bryson, K.-M. (2010). Project performance development framework: An approach for developing performance criteria \& measures for information systems (IS) projects. International Journal of Production Economics, 124(1), 272-292.

Baskerville, R. L., \& Myers, M. D. (2002). Information systems as a reference discipline. MIS Quarterly, 1-14.

Berente, N., Vandenbosch, B., \& Aubert, B. (2009). Information flows and business process integration. Business Process Management Journal, 15(1), 119-141.

Bonabeau, E. (2009). Decisions 2.0: The power of collective intelligence. MIT Sloan Management Review, 50(2), 45-52.

Boudreau, K. J., \& Lakhani, K. R. (2013). Using the crowd as an innovation partner. Harvard business review, 91(4), 60-69.

Bozzon, A., Brambilla, M., Ceri, S., \& Mauri, A. (2013). Reactive crowdsourcing. Proceedings of the 22nd international conference on World wide web, 153-164. 
Brabham, D. C. (2008a). Crowdsourcing as a Model for Problem Solving: An Introduction and Cases. Convergence: The International Journal of Research into New Media Technologies, 14(1), 75-90.

Brabham, D. C. (2008b). Moving the crowd at iStockphoto: The composition of the crowd and motivations for participation in a crowdsourcing application. First monday, 13(6).

Brabham, D. C. (2009). Crowdsourcing the Public Participation Process for Planning Projects. Planning Theory, 8(3), 242-262.

Brabham, D. C. (2010). Moving the crowd at Threadless. Information, Communication \& Society, 13(8), 1122-1145.

Brabham, D. C. (2011). The myth of amateur crowds. Information, Communication \& Society, 15(3), 394-410.

Brabham, D. C. (2012a). A Model for Leveraging Online Communities. In A. Delwiche \& J. Henderson (Eds.), The Participatory Cultures Handbook. New York: Routledge.

Brabham, D. C. (2012b). Motivations for Participation in a Crowdsourcing Application to Improve Public Engagement in Transit Planning. Journal of Applied Communication Research, 40(3), 307-328.

Brabham, D. C. (2013). Crowdsourcing. Canbridge, MA: The MIT Press.

Brabham, D. C., Ribisl, K. M., Kirchner, T. R., \& Bernhardt, J. M. (2014). Crowdsourcing Applications for Public Health. American Journal of Preventive Medicine, 46(2), 179-187.

Brooks, J., McCluskey, S., Turley, E., \& King, N. (2015). The utility of template analysis in qualitative psychology research. Qualitative Research in Psychology, 12(2), 202-222.

Buecheler, T., Sieg, J. H., Füchslin, R. M., \& Pfeifer, R. (2010). Crowdsourcing, open innovation and collective intelligence in the scientific method: a research agenda and operational framework. Proceedings of the twelfth international conference on the synthesis and simulation of living systems, Odense, Denmark.

Burger-Helmchen, T., \& Pénin, J. (2010). The limits of crowdsourcing inventive activities: What do transaction cost theory and the evolutionary theories of the firm teach us. Paper presented at the Workshop on Open Source Innovation, Strasbourg, France.

Carlsson, S. A., Henningsson, S., Hrastinski, S., \& Keller, C. (2011). Socio-technical IS design science research: developing design theory for IS integration management. Information Systems and e-Business Management, 9(1), 109-131. 
Chanal, V., \& Caron-Fasan, M. L. (2010). The difficulties involved in developing business models open to innovation communities: the case of a crowdsourcing platform.M@n@gement, 13(4),318-340.

Chandler, D., \& Kapelner, A. (2013). Breaking monotony with meaning: Motivation in crowdsourcing markets. Journal of Economic Behavior \& Organization, 90, 123133.

Chandrasekaran, B., Josephson, J. R., \& Benjamins, V. R. (1999). What are ontologies, and why do we need them? IEEE Intelligent Systems and their Applications, 14(1), 20-26.

Chatzimilioudis, G., Konstantinidis, A., Laoudias, C., \& Zeinalipour-Yazti, D. (2012). Crowdsourcing with smartphones. Internet Computing, IEEE, 16(5), 36-44.

Chen, C. C., Chen, K., Hsu, C.-Y., \& Li, Y.-C. J. (2011). Developing guideline-based decision support systems using protégé and jess. Computer Methods and Programs in Biomedicine, 102(3), 288-294.

Chesbrough, H. (2013). Open business models: How to thrive in the new innovation landscape: Harvard Business Press.

Chilton, L. B., Horton, J. J., Miller, R. C., \& Azenkot, S. (2010). Task search in a human computation market. Proceedings of the ACM SIGKDD workshop on human computation, 1-9.

Chilton, L. B., Little, G., Edge, D., Weld, D. S., \& Landay, J. A. (2013). Cascade: Crowdsourcing taxonomy creation. Proceedings of the SIGCHI Conference on Human Factors in Computing Systems, 1999-2008.

Cooper, R. G. (2008). Perspective: The Stage-Gate ${ }^{\circledR}$ idea-to-launch process-Update, what's new, and NexGen systems*. Journal of Product Innovation Management, 25(3), 213-232.

Corcho, O., López, M. F., \& Gómez-Pérez, A. (2003). Methodologies, tools and languages for building ontologies. Where is their meeting point? Data \& Knowledge Engineering, 46(1), 41-64.

Corney, J., Torres-Sanchez, C., Jagadeesan, A. P., Yan, X., Regli, W., \& Medellin, H. (2010). Putting the crowd to work in a knowledge-based factory. Advanced Engineering Informatics, 24(3), 243-250.

Cross, N. (1982). Designerly ways of knowing. Design studies, 3(4), 221-227.

Currim, F., \& Ram, S. (2012). Modeling spatial and temporal set-based constraints during conceptual database design. Information Systems Research, 23(1), 109-128.

Dai, P., Lin, C. H., \& Weld, D. S. (2013). POMDP-based control of workflows for crowdsourcing. Artificial Intelligence, 202, 52-85. 
Delir Haghighi, P., Burstein, F., Zaslavsky, A., \& Arbon, P. (2013). Development and evaluation of ontology for intelligent decision support in medical emergency management for mass gatherings. Decision Support Systems, 54(2), 1192-1204.

Dennis, A. R., \& Valacich, J. S. (2001). Conducting experimental research in information systems. Communications of the Association for Information Systems, 7(1).

Dennis, A. R., \& Valacich, J. S. (2014). A Replication Manifesto. AIS Transactions on Replication Research, 1(1), 1-4.

Denyer, D., \& Tranfield, D. (2006). Using qualitative research synthesis to build an actionable knowledge base. Management Decision, 44(2), 213-227.

Dibbern, J., Goles, T., Hirschheim, R., \& Jayatilaka, B. (2004). Information systems outsourcing: a survey and analysis of the literature. ACM SIGMIS Database, 35(4), 6-102.

DiPalantino, D., \& Vojnovic, M. (2009). Crowdsourcing and all-pay auctions. Proceedings of the 10th ACM conference on Electronic commerce, 119-128.

Djelassi, S., \& Decoopman, I. (2013). Customers' participation in product development through crowdsourcing: Issues and implications. Industrial Marketing Management, 42(5), 683-692.

Doan, A., Ramakrishnan, R., \& Halevy, A. Y. (2011). Crowdsourcing systems on the world-wide web. Communications of the ACM, 54(4), 86-96.

Durward, D., Blohm, I., \& Leimeister, J. M. (2016). Crowd Work. Business \& Information Systems Engineering, [Online first], 1-6. doi:10.1007/s12599-0160438-0

Eickhoff, C., \& De Vries, A. (2013). Increasing cheat robustness of crowdsourcing tasks. Information Retrieval, 16(2), 121-137.

Estellés-Arolas, E., \& González-Ladrón-de-Guevara, F. (2012). Towards an integrated crowdsourcing definition. Journal of Information science, 38(2), 189-200.

Estellés-Arolas, E., Navarro-Giner, R., \& González-Ladrón-de-Guevara, F. (2015). Crowdsourcing Fundamentals: Definition and Typology Advances in Crowdsourcing (pp. 33-48): Springer.

Fan, S., Hua, Z., Storey, V. C., \& Zhao, J. L. (2016). A process ontology based approach to easing semantic ambiguity in business process modeling. Data \& Knowledge Engineering, 102, 57-77.

Feller, J., Finnegan, P., Hayes, J., \& O’Reilly, P. (2012). 'Orchestrating'sustainable crowdsourcing: A characterisation of solver brokerages. The Journal of Strategic Information Systems, 21(3), 216-232.

Fettke, P., \& Loos, P. (2003). Classification of reference models: a methodology and its application. Information Systems and e-Business Management, 1(1), 35-53. 
Fonseca, F., \& Martin, J. (2007). Learning the differences between ontologies and conceptual schemas through ontology-driven information systems. Journal of the Association for Information Systems, 8(2), Article 2.

Fortuna, B., Grobelnik, M., \& Mladenic, D. (2007). OntoGen: Semi-automatic Ontology Editor. In M. Smith \& G. Salvendy (Eds.), Human Interface and the Management of Information. Interacting in Information Environments (Vol. 4558, pp. 309318): Springer.

Freyne, J., Coyle, L., Smyth, B., \& Cunningham, P. (2010). Relative status of journal and conference publications in computer science. Communications of the ACM, 53(11), 124-132.

Gadiraju, U., Kawase, R., \& Dietze, S. (2014). A taxonomy of microtasks on the web. Proceedings of the 25th ACM conference on Hypertext and social media, 218223.

Geiger, D., Rosemann, M., Fielt, E., \& Schader, M. (2012). Crowdsourcing Information Systems - Definition, Typology, and Design. Proceedings of the 33rd International Conference on Information Systems.

Geiger, D., \& Schader, M. (2014). Personalized task recommendation in crowdsourcing information systems - Current state of the art. Decision Support Systems, 65, 316.

Geiger, D., Seedorf, S., Schulze, T., Nickerson, R. C., \& Schader, M. (2011). Managing the crowd: towards a taxonomy of crowdsourcing processes. Proceedings of the Seventeenth Americas Conference on Information Systems, Paper 430.

Gennari, J. H., Musen, M. A., Fergerson, R. W., Grosso, W. E., Crubézy, M., Eriksson, H., Noy, N. F., \& Tu, S. W. (2003). The evolution of Protégé: an environment for knowledge-based systems development. International Journal of Humancomputer studies, 58(1), 89-123.

Giachetti, R. E. (2004). A framework to review the information integration of the enterprise. International Journal of Production Research, 42(6), 1147-1166.

Gibson, M., \& Arnott, D. (2007). The use of focus groups in design science research. ACIS 2007 Proceedings, Paper 14.

Gill, A. Q., Alam, S. L., \& Eustace, J. (2015). Social Architecture: An Emergency Management Case Study. Australasian Journal of Information Systems, 19, 2340.

Gomes, C., Schneider, D., Moraes, K., \& de Souza, J. (2012). Crowdsourcing for music: Survey and taxonomy. 2012 IEEE International Conference on Systems, Man, and Cybernetics (SMC), 832-839. 
Gonnokami, K., Morishima, A., \& Kitagawa, H. (2013). Condition-Task-Store: A Declarative Abstraction for Microtask-based Complex Crowdsourcing. Proceedings of the 1st VLDB Workshop on Databases and Crowdsourcing, 2025.

Grace, K., Maher, M. L., Preece, J., Yeh, T., Stangle, A., \& Boston, C. (2015). A Process Model for Crowdsourcing Design: A Case Study in Citizen Science. In J. S. Gero \& S. Hanna (Eds.), Design Computing and Cognition'14 (pp. 245-262): Springer.

Gregor, S. (2006). The nature of theory in information systems. MIS Quarterly, 30(3), 611-642.

Gregor, S., \& Hevner, A. R. (2013). Positioning and presenting design science research for maximum impact. MIS Quarterly, 37(2), 337-355.

Gregor, S., \& Jones, D. (2007). The anatomy of a design theory. Journal of the Association for Information Systems, 8(5), 312-335.

Guarino, N., Oberle, D., \& Staab, S. (2009). What is an Ontology? In S. Staab \& R. Studer (Eds.), Handbook on ontologies (pp. 1-17): Springer.

Guo, T., Schwartz, D. G., Burstein, F., \& Linger, H. (2009). Codifying collaborative knowledge: using Wikipedia as a basis for automated ontology learning. Knowledge Management Research \& Practice, 7(3), 206-217.

Hasselbring, W. (2000). Information system integration. Communications of the ACM, 43(6), 32-38.

Haythornthwaite, C. (2009). Crowds and Communities: Light and Heavyweight Models of Peer Production. 42nd Hawaii International Conference on System Sciences HICSS '09, 1-10.

Heimerl, K., Gawalt, B., Chen, K., Parikh, T., \& Hartmann, B. (2012). CommunitySourcing: engaging local crowds to perform expert work via physical kiosks. Proceedings of the 2012 ACM annual conference on Human Factors in Computing Systems, 1539-1548.

Hetmank, L. (2013). Components and Functions of Crowdsourcing Systems-A Systematic Literature Review. Paper presented at the 11th International Conference on Wirtschaftsinformatik, Leipzig, Germany.

Hetmank, L. (2014). Developing an Ontology for Enterprise Crowdsourcing. Paper presented at the Multikonferenz Wirtschaftsinformatik, Paderborn.

Hevner, A. (2007). The three cycle view of design science research. Scandinavian Journal of Information Systems, 19(2), 87.

Hevner, A., \& Chatterjee, S. (2010). Design Research in Information Systems: Theory and Practice. Integrated Series in Information Systems (Vol. 22): Springer Berlin Heidelberg. 
Hevner, A., March, S. T., Park, J., \& Ram, S. (2004). Design science in information systems research. MIS Quarterly, 28(1), 75-105.

Hirth, M., Hoßfeld, T., \& Tran-Gia, P. (2011). Anatomy of a crowdsourcing platformusing the example of microworkers.com. Paper presented at the Fifth International Conference on Innovative Mobile and Internet Services in Ubiquitous Computing (IMIS), Seoul

Hirth, M., Hoßfeld, T., \& Tran-Gia, P. (2012). Analyzing costs and accuracy of validation mechanisms for crowdsourcing platforms. Mathematical and Computer Modelling, 57(11-12), 2918-2932.

Holsapple, C. W. (2008). DSS architecture and types. In F. Burstein \& C. W. Holsapple (Eds.), Handbook on Decision Support Systems 1 (pp. 163-189): Springer.

Hosack, B., Hall, D., Paradice, D., \& Courtney, J. F. (2012). A look toward the future: decision support systems research is alive and well. Journal of the Association for Information Systems, 13(5), 315-340.

Hossain, M. (2012). Users' motivation to participate in online crowdsourcing platforms. Paper presented at the 2012 International Conference on Innovation Management and Technology Research (ICIMTR).

Hossain, M., Kauranen, I., \& Busi, M. (2015). Crowdsourcing: a comprehensive literature review. Strategic Outsourcing: An International Journal, 8(1), 2-22.

Hosseini, M., Phalp, K., Taylor, J., \& Ali, R. (2014). The four pillars of crowdsourcing: A reference model. 2014 IEEE Eighth International Conference on Research Challenges in Information Science (RCIS), 1-12.

Hosseini, M., Phalp, K., Taylor, J., \& Ali, R. (2015a). On the Configuration of Crowdsourcing Projects. International Journal of Information System Modeling and Design (IJISMD), 6(3), 27-45.

Hosseini, M., Shahri, A., Phalp, K., Taylor, J., \& Ali, R. (2015b). Crowdsourcing: A taxonomy and systematic mapping study. Computer Science Review, 17, 43-69.

Hoßfeld, T., Keimel, C., Hirth, M., Gardlo, B., Habigt, J., Diepold, K., \& Tran-Gia, P. (2013). Best Practices for QoE Crowdtesting: QoE Assessment with Crowdsourcing. IEEE Transactions on Multimedia, 16(2), 541-558.

Höst, M., Regnell, B., \& Wohlin, C. (2000). Using students as subjects - a comparative study of students and professionals in lead-time impact assessment. Empirical Software Engineering, 5(3), 201-214.

Howe, J. (2006a, June). Crowdsourcing: A Definition. Crowdsourcing: Tracking the Rise of the Amateur (Weblog). Retrieved from http://crowdsourcing.typepad.com/cs/2006/06/crowdsourcing_a.html

Howe, J. (2006b). The rise of crowdsourcing. Wired Magazine 2006, 14, 1-4. 
Howe, J. (2008). Crowdsourcing: How the power of the crowd is driving the future of business: Century.

Huberman, B. A., Romero, D. M., \& Wu, F. (2009). Crowdsourcing, attention and productivity. Journal of Information science, 35(6), 758-765.

Huston, L., \& Sakkab, N. (2006). Connect and develop. Harvard business review, 84(3), 58-66.

Huysmans, J., Dejaeger, K., Mues, C., Vanthienen, J., \& Baesens, B. (2011). An empirical evaluation of the comprehensibility of decision table, tree and rule based predictive models. Decision Support Systems, 51(1), 141-154.

Iivari, J. (2007). A paradigmatic analysis of information systems as a design science. Scandinavian Journal of Information Systems, 19(2), Article 5.

Ipeirotis, P. G., Provost, F., \& Wang, J. (2010). Quality management on amazon mechanical turk. Proceedings of the ACM SIGKDD workshop on human computation, 64-67.

Jabareen, Y. (2009). Building a conceptual framework: philosophy, definitions, and procedure. International Journal of Qualitative Methods, 8(4), 49-62.

Jeppesen, L. B., \& Lakhani, K. R. (2010). Marginality and problem-solving effectiveness in broadcast search. Organization Science, 21(5), 1016-1033.

Jonker, J., \& Pennink, B. W. (2010). Conceptual Models The Essence of Research Methodology (pp. 43-63): Springer Berlin Heidelberg.

Kannangara, S. N., \& Uguccioni, P. (2013). Risk Management in Crowdsourcing-Based Business Ecosystems. Technology Innovation Management Review 3(12), 32-38.

Karger, D. R., Oh, S., \& Shah, D. (2013). Efficient crowdsourcing for multi-class labeling. ACM SIGMETRICS Performance Evaluation Review, 41(1), 81-92.

Katz, R., \& Allen, T. J. (1982). Investigating the Not Invented Here (NIH) syndrome: A look at the performance, tenure, and communication patterns of $50 \mathrm{R} \& \mathrm{D}$ Project Groups. $R \& D$ Management, 12(1), 7-20.

Kaufmann, N., Schulze, T., \& Veit, D. (2011). More than fun and money. Worker motivation in crowdsourcing-a study on mechanical turk. Proceedings of the Seventeenth Americas Conference on Information Systems, Detroit, MI, Paper 340.

Kazai, G. (2010). An Exploration of the Influence that Task Parameters have on the Performance of Crowds. Proceedings of the CrowdConf 2010.

Kazai, G., Kamps, J., \& Milic-Frayling, N. (2011). Worker types and personality traits in crowdsourcing relevance labels. Proceedings of the 20th ACM international conference on Information and knowledge management, 1941-1944. 
Kazman, R., \& Chen, H. M. (2009). The metropolis model a new logic for development of crowdsourced systems. Communications of the ACM, 52(7), 76-84.

Khazankin, R., Psaier, H., Schall, D., \& Dustdar, S. (2011). QoS-Based Task Scheduling in Crowdsourcing Environments. In G. Kappel, Z. Maamar, \& H. MotahariNezhad (Eds.), Service-Oriented Computing (Vol. 7084, pp. 297-311): Springer.

Khazankin, R., Satzger, B., \& Dustdar, S. (2012a). Optimized execution of business processes on crowdsourcing platforms. IEEE 8th International Conference on Collaborative Computing: Networking, Applications and Worksharing, 443 - 451.

Khazankin, R., Satzger, B., \& Dustdar, S. (2012b). Predicting QoS in Scheduled Crowdsourcing. In J. Ralyté, X. Franch, S. Brinkkemper, \& S. Wrycza (Eds.), Advanced Information Systems Engineering (Vol. 7328, pp. 460-472): Springer Berlin Heidelberg.

King, N. (2004). Using templates in the thematic analysis of texts. In C. Cassell \& G. Symon (Eds.), Essential guide to qualitative methods in organizational research (pp. 256-270): SAGE Publications.

King, N. (2012). Doing template analysis. Qualitative organizational research: Core methods and current challenges, 426-450.

Kitchenham, B. (2007). Guidelines for performing systematic literature reviews in software engineering. Ver. 2.3 EBSE Technical Report.

Kittur, A., Nickerson, J., Bernstein, M., Gerber, E., Shaw, A., Zimmerman, J., Lease, M., \& Horton, J. (2013). The Future of Crowd Work. Proceedings of the 2013 Conference on Computer Supported Cooperative Work.

Kittur, A., Smus, B., Khamkar, S., \& Kraut, R. E. (2011). Crowdforge: Crowdsourcing complex work. Proceedings of the 24th annual ACM symposium on User interface software and technology, 43-52.

Kleemann, F., Voß, G. G., \& Rieder, K. (2008). Un (der) paid Innovators: The Commercial Utilization of Consumer Work through Crowdsourcing. Science, Technology \& Innovation Studies, 4(1), 5-26.

Kohlborn, T. (2012). Identification and evaluation of service bundles for governmental one-stop portals. (Doctor of Philosophy), Queensland University of Technology.

Kordon, F. (2002). An introduction to rapid system prototyping. IEEE Transactions on Software Engineering, 28(9), 817-821.

Krueger, R. A., \& Casey, M. A. (2014). Focus groups: A practical guide for applied research: Sage publications.

Kucherbaev, P., Tranquillini, S., Daniel, F., Casati, F., Marchese, M., Brambilla, M., \& Fraternali, P. (2013). Business Processes for the Crowd Computer. In M. L. Rosa 
\& P. Soffer (Eds.), Business Process Management Workshops (pp. 256-267): Springer Berlin Heidelberg.

Küçük, D., \& Arslan, Y. (2014). Semi-automatic construction of a domain ontology for wind energy using Wikipedia articles. Renewable Energy, 62, 484-489.

Kulkarni, A., Can, M., \& Hartmann, B. (2012). Collaboratively crowdsourcing workflows with turkomatic. Proceedings of the ACM 2012 Conference on Computer Supported Cooperative Work, 1003-1012.

La Vecchia, G., \& Cisternino, A. (2010). Collaborative Workforce, Business Process Crowdsourcing as an Alternative of BPO. In F. Daniel \& F. M. Facca (Eds.), ICWE2010. LNCS (Vol. 6385, pp. 425-430): Springer.

Lacity, M. C., Solomon, S., Yan, A., \& Willcocks, L. P. (2011). Business process outsourcing studies: a critical review and research directions. Journal of information technology, 26(4), 221-258.

LaToza, T. D., \& Hoek, A. v. d. (2016). Crowdsourcing in Software Engineering: Models, Motivations, and Challenges. IEEE Software, 33(1), 74-80. doi:10.1109/MS.2016.12

Le, J., Edmonds, A., Hester, V., \& Biewald, L. (2010). Ensuring quality in crowdsourced search relevance evaluation: The effects of training question distribution. Paper presented at the SIGIR 2010 workshop on crowdsourcing for search evaluation.

Leimeister, J. M., Huber, M., Bretschneider, U., \& Krcmar, H. (2009). Leveraging Crowdsourcing: Activation-Supporting Components for IT-Based Ideas Competition. Journal of Management Information Systems, 26(1), 197-224.

Levac, D., Colquhoun, H., \& O'Brien, K. K. (2010). Scoping studies: advancing the methodology. Implement Science, 5(1), 1-9.

Levy, Y., \& Ellis, T. J. (2006). A systems approach to conduct an effective literature review in support of information systems research. Informing Science: International Journal of an Emerging Transdiscipline, 9, 181-212.

Lim, Y.-K., Stolterman, E., \& Tenenberg, J. (2008). The anatomy of prototypes: Prototypes as filters, prototypes as manifestations of design ideas. ACM Transactions on Computer-Human Interaction (TOCHI), 15(2), Article 7.

Little, G., Chilton, L. B., Goldman, M., \& Miller, R. C. (2010). Turkit: human computation algorithms on mechanical turk. Proceedings of the 23nd annual ACM symposium on User interface software and technology, 57-66.

Lloret, E., Plaza, L., \& Aker, A. (2012). Analyzing the capabilities of crowdsourcing services for text summarization. Language Resources and Evaluation, 47(2), 337369. 
Lofi, C., Selke, J., \& Balke, W. T. (2012). Information extraction meets crowdsourcing: A promising couple. Datenbank-Spektrum, 12(2), 109-120.

Lopez, M., Vukovic, M., \& Laredo, J. (2010). PeopleCloud Service for Enterprise Crowdsourcing. 2010 IEEE International Conference on Services Computing (SCC), 538-545.

López, M. F., Gómez-Pérez, A., \& Corcho, O. (2004). Ontological Engineering: with examples from the areas of Knowledge Management, e-Commerce and the Semantic Web: Springer.

Lu, B., Hirschheim, R., \& Schwarz, A. (2015). Examining the antecedent factors of online microsourcing. Information Systems Frontiers, 17(3), 601-617.

Lüttgens, D., Pollok, P., Antons, D., \& Piller, F. (2014). Wisdom of the crowd and capabilities of a few: internal success factors of crowdsourcing for innovation. Journal of Business Economics, 84(3), 339-374.

Luz, N., Silva, N., \& Novais, P. (2015). A survey of task-oriented crowdsourcing. Artificial Intelligence Review, 44(2), 187-213.

Majchrzak, A., \& Malhotra, A. (2013). Towards an information systems perspective and research agenda on crowdsourcing for innovation. The Journal of Strategic Information Systems, 22(4), 257-268.

Malone, T. W., Laubacher, R., \& Dellarocas, C. (2010). The collective intelligence genome. IEEE Engineering Management Review, 38(3), 38- 52.

Man-Ching, Y., King, I., \& Kwong-Sak, L. (2011). A Survey of Crowdsourcing Systems. 2011 IEEE third international conference on Privacy, security, risk and trust (passat), and 2011 IEEE third international conference on social computing (socialcom), 766-773.

March, S. T., \& Smith, G. F. (1995). Design and natural science research on information technology. Decision Support Systems, 15(4), 251-266.

Marjanovic, S., Fry, C., \& Chataway, J. (2012). Crowdsourcing based business models: In search of evidence for innovation 2.0. Science and Public Policy, 39(3), 318332.

Marsh, H. W. (2007). Students' evaluations of university teaching: Dimensionality, reliability, validity, potential biases and usefulness. In R. P. Perry \& J. C. Smart (Eds.), The scholarship of teaching and learning in higher education: An evidence-based perspective (pp. 319-383): Springer.

Martineau, E. (2012). A typology of crowdsourcing participation styles. (Master Thesis), Concordia University.

Mason, W., \& Suri, S. (2012). Conducting behavioral research on Amazon's Mechanical Turk. Behavior research methods, 44(1), 1-23. 
Mason, W., \& Watts, D. J. (2009). Financial incentives and the "performance of crowds". Proceedings of the ACM SIGKDD Workshop on Human Computation, 77-85.

Mendling, J., Strembeck, M., \& Recker, J. (2012). Factors of process model comprehension-findings from a series of experiments. Decision Support Systems, 53(1), 195-206.

Mettler, T., Eurich, M., \& Winter, R. (2014). On the use of experiments in design science research: a proposition of an evaluation framework. Communications of the Association for Information Systems, 34(1), 223-240.

Miah, S. J. (2008). An Ontology Based Design Environment for Rural Business Decision Support. (Doctor of Philosophy), Griffith University, Brisbane, Australia.

Miah, S. J., Gammack, J., \& Kerr, D. (2007). Ontology development for context-sensitive decision support. Third International Conference on Semantics, Knowledge and Grid, 475-478.

Miah, S. J., Kerr, D., \& von Hellens, L. (2014). A collective artefact design of decision support systems: design science research perspective. Information Technology \& People, 27(3), 259-279.

Miah, S. J., Kerr, D. V., \& Gammack, J. G. (2009). A methodology to allow rural extension professionals to build target-specific expert systems for Australian rural business operators. Expert Systems with Applications, 36(1), 735-744.

Miles, M. B., Huberman, A. M., \& Saldaña, J. (2014). Qualitative data analysis: A methods sourcebook: SAGE Publications, Incorporated.

Mingers, J. (2003). The paucity of multimethod research: a review of the information systems literature. Information Systems Journal, 13(3), 233-249.

Montgomery, C. D. (2012). Design and Analysis of Experiments (8 ed.). New York: John Willey \& Sons, Inc.

Muhdi, L., Daiber, M., Friesike, S., \& Boutellier, R. (2011). The crowdsourcing process: an intermediary mediated idea generation approach in the early phase of innovation. International Journal of Entrepreneurship and Innovation Management, 14(4), 315-332.

Muntés-Mulero, V., Paladini, P., Manzoor, J., Gritti, A., Larriba-Pey, J. L., \& Mijnhardt, F. (2013). Crowdsourcing for industrial problems. In J. Nin \& D. Villatoro (Eds.), Citizen in Sensor Networks. LNCS (Vol. 7685, pp. 6-18): Springer Berlin Heidelberg.

Nakatsu, R. T., Grossman, E. B., \& Iacovou, C. L. (2014). A taxonomy of crowdsourcing based on task complexity. Journal of Information Science, 40(6), 823-834.

Naroditskiy, V., Jennings, N. R., Van Hentenryck, P., \& Cebrian, M. (2013). Crowdsourcing dilemma. arXiv preprint arXiv:1304.3548. 
Nemati, H. R., Steiger, D. M., Iyer, L. S., \& Herschel, R. T. (2002). Knowledge warehouse: an architectural integration of knowledge management, decision support, artificial intelligence and data warehousing. Decision Support Systems, 33(2), 143-161.

Nickerson, R. C., Varshney, U., \& Muntermann, J. (2012). A method for taxonomy development and its application in information systems. European Journal of Information Systems, 22(3), 336-359.

Niederman, F., \& March, S. T. (2012). Design science and the accumulation of knowledge in the information systems discipline. ACM Transactions on Management Information Systems (TMIS), 3(1), Article 1.

Niehaves, B. (2007). On episemological diversity in design science: New vistas for a design-oriented IS research? ICIS 2007 Proceedings, Paper 133.

Nielsen, J. (2006, October). Participation inequality: Encouraging more users to contribute. Jakob Nielsen's alertbox. Retrieved from https://www.nngroup.com/articles/participation-inequality/

Okoli, C. (2015). A Guide to Conducting a Standalone Systematic Literature Review. Communications of the Association for Information Systems, 37(1), Article 43.

Okoli, C., \& Schabram, K. (2010). A guide to conducting a systematic literature review of information systems research. Sprouts: Working Papers on Information Systems, 10(26).

OReilly, T. (2007). What is Web 2.0: Design patterns and business models for the next generation of software. Communications \& strategies(1), 17.

Orlikowski, W. J., \& Baroudi, J. J. (1991). Studying information technology in organizations: Research approaches and assumptions. Information systems research, 2(1), 1-28.

Osterwalder, A. (2004). The business model ontology: A proposition in a design science approach. (Doctor of Philosophy), Institut d'Informatique et Organisation. Lausanne, Switzerland, University of Lausanne, Ecole des Hautes Etudes Commerciales HEC.

Osterwalder, A., \& Pigneur, Y. (2002). An eBusiness model ontology for modeling eBusiness. BLED 2002 Proceeding, Paper 2.

Osterwalder, A., \& Pigneur, Y. (2004). An ontology for e-business models. Value creation from e-business models, 65-97.

Ostrowski, L., Helfert, M., \& Gama, N. (2014). Ontology engineering step in design science research methodology: a technique to gather and reuse knowledge. Behaviour \& Information Technology, 33(5), 443-451. 
Palacios, M., Martinez-Corral, A., Nisar, A., \& Grijalvo, M. (2016). Crowdsourcing and organizational forms: Emerging trends and research implications. Journal of Business Research, 69(5), 1834-1839.

Paolacci, G., Chandler, J., \& Ipeirotis, P. (2010). Running experiments on amazon mechanical turk. Judgment and Decision Making, 5(5), 411-419.

Paré, G., Trudel, M.-C., Jaana, M., \& Kitsiou, S. (2015). Synthesizing information systems knowledge: A typology of literature reviews. Information \& Management, 52, 183-199.

Park, S., Shoemark, P., \& Morency, L.-P. (2014). Toward crowdsourcing micro-level behavior annotations: the challenges of interface, training, and generalization. Proceedings of the 19th international conference on Intelligent User Interfaces, 37-46.

Pedersen, J., Kocsis, D., Tripathi, A., Tarrell, A., Weerakoon, A., Tahmasbi, N., Jie, X., Wei, D., Onook, O., \& De Vreede, G. J. (2013). Conceptual Foundations of Crowdsourcing: A Review of IS Research. 46th Hawaii International Conference on System Sciences (HICSS), 579-588.

Peffers, K., Rothenberger, M., Tuunanen, T., \& Vaezi, R. (2012). Design science research evaluation. In K. Peffers, M. Rothenberger, \& B. Kuechler (Eds.), Design Science Research in Information Systems. Advances in Theory and Practice. LNCS (Vol. 7286, pp. 398-410): Springer Berlin Heidelberg.

Peffers, K., Tuunanen, T., Rothenberger, M. A., \& Chatterjee, S. (2007). A design science research methodology for information systems research. Journal of Management Information Systems, 24(3), 45-77.

Pfeiffer, J., Benbasat, I., \& Rothlauf, F. (2014). Minimally Restrictive Decision Support Systems. ICIS 2014 Proceedings, Paper 36.

Pinto, H. S., \& Martins, J. P. (2004). Ontologies: How can they be built? Knowledge and Information Systems, 6(4), 441-464.

Poetz, M. K., \& Schreier, M. (2012). The Value of Crowdsourcing: Can Users Really Compete with Professionals in Generating New Product Ideas? Journal of Product Innovation Management, 29(2), 245-256.

Pries-Heje, J., \& Baskerville, R. (2008). The design theory nexus. MIS Quarterly, 32(4), 731-755.

Prokesch, T., \& Wohlenberg, H. (2014). Results From A Group Wisdom Supporting System. Proceedings of the European Conference on Information Systems (ECIS) 2014, Paper 7. 
Prpić, J., Shukla, P. P., Kietzmann, J. H., \& McCarthy, I. P. (2015). How to work a crowd: Developing crowd capital through crowdsourcing. Business Horizons, 58(1), 7785.

Quinn, A. J., \& Bederson, B. B. (2011). Human computation: a survey and taxonomy of a growing field. Proceedings of the SIGCHI conference on human factors in computing systems, 1403-1412.

Ranade, G., \& Varshney, L. R. (2012). To Crowdsource or not to Crowdsource? Paper presented at the AAAI Workshop Human Comput. (HCOMP'12).

Rhee, C., \& Rao, R. H. (2008). Evaluation of Decision Support Systems. In F. Burstein \& C. W. Holsapple (Eds.), Handbook on Decision Support Systems 2: Variations (pp. 313-327). Berlin, Heidelberg: Springer Berlin Heidelberg.

Ridge, M. M. (2014). Crowdsourcing our Cultural Heritage: Ashgate Publishing, Ltd.

Rockwell, J. A., Grosse, I. R., Krishnamurty, S., \& Wileden, J. C. (2010). A semantic information model for capturing and communicating design decisions. Journal of Computing and Information Science in Engineering, 10(3), 1-8.

Rosen, P. A. (2011). Crowdsourcing Lessons for Organizations. Journal of Decision Systems, 20(3), 309-324.

Rouse, A. C. (2010). A preliminary taxonomy of crowdsourcing. Proceedings of the 21st Australasian Conference on Information Systems, Paper 76.

Sakamoto, Y., Tanaka, Y., Yu, L., \& Nickerson, J. V. (2011). The crowdsourcing design space. In D. Schmorrow \& C. Fidopiastis (Eds.), Foundations of Augmented Cognition. Directing the Future of Adaptive Systems. LNCS (Vol. 6780, pp. 346355): Springer Berlin Heidelberg.

Saldarini, R. A. (1989). Analysis and design of business information systems: Prentice Hall PTR Upper Saddle River, NJ, USA.

Sánchez, D., \& Moreno, A. (2008). Learning non-taxonomic relationships from web documents for domain ontology construction. Data \& Knowledge Engineering, 64(3), 600-623.

Satzger, B., Psaier, H., Schall, D., \& Dustdar, S. (2011). Stimulating skill evolution in market-based crowdsourcing. In S. Rinderle-Ma, F. Toumani, \& K. Wolf (Eds.), BPM 2011. LNCS (Vol. 6896, pp. 66-82): Springer Berlin Heidelberg.

Satzger, B., Psaier, H., Schall, D., \& Dustdar, S. (2012). Auction-based crowdsourcing supporting skill management. Information Systems, 38(4), 547-560.

Saxton, G. D., Oh, O., \& Kishore, R. (2013). Rules of crowdsourcing: Models, issues, and systems of control. Information Systems Management, 30(1), 2-20.

Schall, D. (2012). Service-oriented crowdsourcing: architecture, protocols and algorithms: Springer Science \& Business Media. 
Schenk, E., \& Guittard, C. (2009). Crowdsourcing: What can be Outsourced to the Crowd, and Why? Paper presented at the Workshop on Open Source Innovation, Strasbourg, France.

Schenk, E., \& Guittard, C. (2011). Towards a characterization of crowdsourcing practices. Journal of Innovation Economics, 7(1), 93-107.

Schulze, T., Seedorf, S., Geiger, D., Kaufmann, N., \& Schader, M. (2011). Exploring task properties in crowdsourcing-an empirical study on mechanical turk. ECIS2011, Paper 122.

Seltzer, E., \& Mahmoudi, D. (2013). Citizen Participation, Open Innovation, and Crowdsourcing Challenges and Opportunities for Planning. Journal of Planning Literature, 28(1), 3-18.

Shanks, G., Tansley, E., \& Weber, R. (2003). Using ontology to validate conceptual models. Communications of the ACM, 46(10), 85-89.

Sharman, R., Kishore, R., \& Ramesh, R. (2004). Computational Ontologies and Information Systems II: Formal Specification. Communications of the Association for Information Systems, 14(1), Article 9.

Shaw, M., \& Garlan, D. (1996). Software architecture: perspectives on an emerging discipline: Prentice Hall Englewood Cliffs.

Simula, H. (2013). The Rise and Fall of Crowdsourcing? 46th Hawaii International Conference on System Sciences (HICSS), 2783-2791.

Sjøberg, D. I., Hannay, J. E., Hansen, O., Kampenes, V. B., Karahasanovic, A., Liborg, N.-K., \& Rekdal, A. C. (2005). A survey of controlled experiments in software engineering. IEEE Transactions on Software Engineering, 31(9), 733-753.

Smith, M., Busi, M., Ball, P., \& Van Der Meer, R. (2008). Factors influencing an organisation's ability to manage innovation: a structured literature review and conceptual model. International Journal of Innovation Management, 12(04), 655676.

Soh, C., Markus, M. L., \& Goh, K. H. (2006). Electronic marketplaces and price transparency: strategy, information technology, and success. MIS Quarterly, 705723.

Sonnenberg, C., \& vom Brocke, J. (2012a). Evaluation Patterns for Design Science Research Artefacts. In M. Helfert \& B. Donnellan (Eds.), Practical Aspects of Design Science (Vol. 286, pp. 71-83): Springer Berlin Heidelberg.

Sonnenberg, C., \& vom Brocke, J. (2012b). Evaluations in the science of the artificialreconsidering the build-evaluate pattern in design science research. In K. Peffers, M. Rothenberger, \& B. Kuechler (Eds.), Design Science Research in Information Systems. Advances in Theory and Practice. LNCS (pp. 381-397): Springer. 
Stewart, O., Lubensky, D., \& Huerta, J. M. (2010). Crowdsourcing participation inequality: a SCOUT model for the enterprise domain. Proceedings of the ACM SIGKDD Workshop on Human Computation, 30-33.

Stol, K.-J., \& Fitzgerald, B. (2014). Two's company, three's a crowd: a case study of crowdsourcing software development. Proceedings of the 36th International Conference on Software Engineering, 187-198.

Surowiecki, J. (2004). The Wisdom of Crowds: Why the Many Are Smarter Than the Few and How Collective Wisdom Shapes Business. New York: Doubleday.

Sutherlin, G. (2013). A voice in the crowd: Broader implications for crowdsourcing translation during crisis. Journal of Information science, 39(3), 397-409.

Tetreault, J., Chodorow, M., \& Madnani, N. (2014). Bucking the trend: improved evaluation and annotation practices for ESL error detection systems. Language Resources and Evaluation, 48(1), 5-31.

Threadless. (2015). Threadless help center. Retrieved from http://support.threadless.com

Thuan, N. H., Antunes, P., \& Johnstone, D. (2015a). A Design Science Method for Emerging Decision Support Environments. Proceedings of the Australasian Conference on Information Systems 2015, Paper 32.

Thuan, N. H., Antunes, P., \& Johnstone, D. (2016). Factors Influencing the Decision to Crowdsource: a Systematic Literature Review. Information Systems Frontiers, 18(1), 47-68.

Thuan, N. H., Antunes, P., Johnstone, D., \& Ha, X. S. (2015b). Building an Enterprise Ontology of Business Process Crowdsourcing: A Design Science Approach. PACIS 2015 Proceedings. AISeL, Paper 112.

Tokarchuk, O., Cuel, R., \& Zamarian, M. (2012). Analyzing Crowd Labor and Designing Incentives for Humans in the Loop. IEEE Internet Computing Magazine, 16(5), 45-51.

Tomlinson, B., Ross, J., Paul, A., Eric, B., Donald, P., Joseph, C., Martin, M., Syavash, N., Marco, L., Birgit, P., Andrew, T., David, C., Gary, O., Six, S., Marcus, S., Fabio, R. P., Albert, A. S., Eric, M., Xavier, F., Florian, F. M., Joseph, J. K., Rebecca, W. B., Marisa, L. C., Patrick, C. S., Johanna, B., Nitesh, G., Pirjo, N., Jeff, H., Nilufar, B., \& Craig, S. (2012). Massively distributed authorship of academic papers. Proceedings of the 2012 ACM annual conference extended abstracts on Human Factors in Computing Systems Extended Abstracts.

Tranquillini, S., Daniel, F., Kucherbaev, P., \& Casati, F. (2015). Modeling, Enacting, and Integrating Custom Crowdsourcing Processes. ACM Transactions on the Web (TWEB), 9(2), Article 7. 
Tremblay, M. C., Hevner, A. R., \& Berndt, D. J. (2010). Focus groups for artifact refinement and evaluation in design research. Communications of the Association for Information Systems, 26, Article 27.

Tremblay, M. C., Hevner, A. R., \& Berndt, D. J. (2012). Design of an information volatility measure for health care decision making. Decision Support Systems, 52(2), 331-341.

Uschold, M., \& King, M. (1995). Towards a methodology for building ontologies. Paper presented at the IJCAI95 Workshop on Basic Ontological Issues in Knowledge Sharing, Montreal.

Valaski, J., Malucelli, A., \& Reinehr, S. (2012). Ontologies application in organizational learning: A literature review. Expert Systems with Applications, 39(8), 7555-7561.

Van Aken, J. E. (2004). Management research based on the paradigm of the design sciences: the quest for field-tested and grounded technological rules. Journal of management studies, 41(2), 219-246.

Van Aken, J. E. (2005). Management research as a design science: articulating the research products of mode 2 knowledge production in management. British Journal of Management, 16(1), 19-36.

Van Aken, J. E., \& Romme, A. G. L. (2012). A design science approach to evidencebased management. In D. M. Rousseau (Ed.), The Oxford handbook of evidencebased management (pp. 43-60).

Van der Aalst, W., \& Hee, K. M. (2004). Workflow management: models, methods, and systems. Cambridge, MA: The MIT Press.

Van Valkenhoef, G., Tervonen, T., Zwinkels, T., De Brock, B., \& Hillege, H. (2013). ADDIS: a decision support system for evidence-based medicine. Decision Support Systems, 55(2), 459-475.

Varshney, U., Nickerson, R., \& Muntermann, J. (2015). Towards the Development of a Taxonomic Theory. AMCIS 2015, Paper 4.

Venable, J. (2006). The role of theory and theorising in design science research. Proceedings of the 1st International Conference on Design Science in Information Systems and Technology (DESRIST 2006), 1-18.

Venable, J., Pries-Heje, J., \& Baskerville, R. (2012). A comprehensive framework for evaluation in design science research. Design Science Research in Information Systems. Advances in Theory and Practice, 423-438.

Venable, J., Pries-Heje, J., \& Baskerville, R. (2016). FEDS: a framework for evaluation in design science research. European Journal of Information Systems, 25(1), 7789. 
Venkatesh, V., \& Davis, F. D. (2000). A theoretical extension of the technology acceptance model: Four longitudinal field studies. Management science, 46(2), 186-204.

Vicente, K. J. (1999). Cognitive work analysis: Toward safe, productive, and healthy computer-based work: CRC Press.

Vogrinčič, S., \& Bosnić, Z. (2011). Ontology-based multi-label classification of economic articles. Computer Science and Information Systems, 8(1), 101-119.

Vukovic, M. (2009). Crowdsourcing for enterprises. Paper presented at the 2009 World Conference on Services-I, Los Angeles, CA.

Vukovic, M., Laredo, J., \& Rajagopal, S. (2010). Challenges and experiences in deploying enterprise crowdsourcing service. In B. Benatallah, F. Casati, G. Kappel, \& G. Rossi (Eds.), ICWE 2010. LNCS (Vol. 6189, pp. 460-467): Springer Berlin Heidelberg.

Vuurens, J. B., \& De Vries, A. P. (2012). Obtaining high-quality relevance judgments using crowdsourcing. IEEE Internet Computing, 16(5), 20-27.

Wand, Y., \& Weber, R. (1995). On the deep structure of information systems. Information Systems Journal, 5(3), 203-223.

Wang, A., Hoang, C. D. V., \& Kan, M.-Y. (2013). Perspectives on crowdsourcing annotations for natural language processing. Language resources and evaluation, 47(1), 9-31.

Webster, J., \& Watson, R. T. (2002). Analyzing the past to prepare for the future: writing a literature review. MIS Quarterly, 26(2), xiii-xxiii.

Westpac. (2013, October). Westpac New Zealand to crowdsource mobile banking apps. Retrieved from http://www.westpac.co.nz/who-we-are/newsroom/mediareleases-2013/westpac-new-zealand-to-crowdsource-mobile-banking-apps/

Wexler, M. N. (2011). Reconfiguring the sociology of the crowd: exploring crowdsourcing. International Journal of Sociology and Social Policy, 31(1/2), 620.

Whitla, P. (2009). Crowdsourcing and its application in marketing activities. Contemporary Management Research, 5(1), 15-28.

Winter, R. (2008). Design science research in Europe. European Journal of Information Systems, 17(5), 470-475.

Winter, R., \& Albani, A. (2013). Restructuring the design science research knowledge base. In R. Baskerville, M. De Marco, \& P. Spagnoletti (Eds.), Designing Organizational Systems (pp. 63-81): Springer.

Wohlin, C., Runeson, P., Höst, M., Ohlsson, M. C., Regnell, B., \& Wesslén, A. (2012). Experimentation in software engineering: Springer Science \& Business Media. 
Wong, W., Liu, W., \& Bennamoun, M. (2012). Ontology learning from text: A look back and into the future. ACM Computing Surveys (CSUR), 44(4), Article 20.

Wu, F., Wilkinson, D. M., \& Huberman, B. A. (2009). Feedback loops of attention in peer production. International Conference on Computational Science and

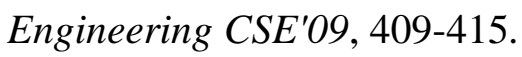

Wu, W., Tsai, W.-T., \& Li, W. (2013). An evaluation framework for software crowdsourcing. Frontiers of Computer Science, 7(5), 694-709.

Wüllenweber, K., Beimborn, D., Weitzel, T., \& König, W. (2008). The impact of process standardization on business process outsourcing success. Information Systems Frontiers, 10(2), 211-224.

Xu, Y., Ribeiro-Soriano, D. E., \& Gonzalez-Garcia, J. (2015). Crowdsourcing, innovation and firm performance. Management Decision, 53(6), 1158-1169.

Yang, J., Adamic, L. A., \& Ackerman, M. S. (2008). Crowdsourcing and knowledge sharing: strategic user behavior on taskcn. Proceedings of the 9th ACM conference on Electronic commerce, 246-255.

Yin, R. K. (2013a). Case study research: Design and methods: Sage publications.

Yin, R. K. (2013b). Validity and generalization in future case study evaluations. Evaluation, 19(3), 321-332.

Zachman, J. A. (1987). A framework for information systems architecture. IBM systems journal, 26(3), 276-292.

Zhao, Y., \& Zhu, Q. (2014). Evaluation on crowdsourcing research: Current status and future direction. Information Systems Frontiers, 16(3), 417-434.

Zheng, H., Li, D., \& Hou, W. (2011). Task Design, Motivation, and Participation in Crowdsourcing Contests. International Journal of Electronic Commerce, 15(4), 57-88.

Zogaj, S., Bretschneider, U., \& Leimeister, J. M. (2014). Managing crowdsourced software testing: a case study based insight on the challenges of a crowdsourcing intermediary. Journal of Business Economics, 84(3), 375-405. 


\section{APPENDICES}

\section{Appendix A. Crowdsourcing Classifications}

This appendix summarises the major crowdsourcing classifications, which was reviewed and summarised in Chapter 2. For each classification, we analysed its purpose, the classified objects, main variables, categories, research methods, and means of representation. The analysis followed the techniques for analysing IS taxonomies proposed by Nickerson et al. (2012) and Varshney et al. (2015). The results are summarised in the following table, which supports our review in Chapter 2.

\begin{tabular}{|c|c|c|c|c|}
\hline $\begin{array}{c}\text { Classification } \\
\text { purpose } \\
\text { Reference }\end{array}$ & \begin{tabular}{|c|} 
Classification \\
objects
\end{tabular} & $\begin{array}{c}\text { Classification } \\
\text { variables }\end{array}$ & Categories/ Types & $\begin{array}{c}\text { Method } \\
\text { Means of } \\
\text { representation } \\
\end{array}$ \\
\hline \multicolumn{5}{|l|}{ Applications } \\
\hline $\begin{array}{l}\text { Examine what } \\
\text { firms utilise } \\
\text { crowdsourcing for } \\
\text { (Whitla, 2009) }\end{array}$ & Applications & $\begin{array}{l}\text { - Function (what } \\
\text { crowdsourcing } \\
\text { is utilised for) }\end{array}$ & $\begin{array}{l}\text { - Product } \\
\text { development } \\
\text { - Advertising and } \\
\text { promotion } \\
\text { - Marketing research }\end{array}$ & $\begin{array}{l}\text { Theoretical; } \\
\text { deductive } \\
\text { Text }\end{array}$ \\
\hline $\begin{array}{l}\text { Group } \\
\text { applications } \\
\text { (Gomes et al., } \\
\text { 2012) }\end{array}$ & Applications & - Function & $\begin{array}{l}\text { - Music co-creation } \\
\text { - Decision support } \\
\text { - Crowdsourced } \\
\text { music collection } \\
\text { and management } \\
\text { (MCM) } \\
\text { - Promoting music } \\
\text { information } \\
\text { - Marketplace } \\
\text { - Crowdfunding }\end{array}$ & $\begin{array}{l}\text { Empirical; } \\
\text { inductive } \\
\text { One-dimensional } \\
\text { table }\end{array}$ \\
\hline $\begin{array}{l}\text { Conceptualise the } \\
\text { field } \\
\text { (Chatzimilioudis, } \\
\text { Konstantinidis, } \\
\text { Laoudias, \& } \\
\text { Zeinalipour-Yazti, } \\
\text { 2012) }\end{array}$ & Applications & $\begin{array}{l}\text { - Web extended } \\
\text { - Involvement } \\
\text { - Data wisdom } \\
\text { - Contribution } \\
\text { quality } \\
\text { - Incentives } \\
\text { - Human skill } \\
\text { - Sensors } \\
\text { - Location }\end{array}$ & $\begin{array}{l}\text { Taxonomy defined } \\
\text { by the combination } \\
\text { of the variables }\end{array}$ & $\begin{array}{l}\text { Not explicitly } \\
\text { stated } \\
\text { One-dimensional } \\
\text { table }\end{array}$ \\
\hline
\end{tabular}




\begin{tabular}{|c|c|c|c|c|}
\hline $\begin{array}{c}\text { Classification } \\
\text { purpose }\end{array}$ & \begin{tabular}{|c|}
$\begin{array}{c}\text { Classification } \\
\text { objects }\end{array}$ \\
\end{tabular} & $\begin{array}{c}\text { Classification } \\
\text { variables }\end{array}$ & Categories/ Types & $\begin{array}{c}\text { Method } \\
\text { Means of } \\
\text { representation }\end{array}$ \\
\hline \begin{tabular}{|l} 
Explore \\
crowdsourcing \\
(Kleemann et al., \\
$2008)$
\end{tabular} & Applications & - Function & $\begin{array}{l}\text { - Product } \\
\text { development and } \\
\text { configuration } \\
\text { - Product design } \\
\text { - Competitive bids } \\
\text { - Permanent open } \\
\text { calls } \\
\text { - Community } \\
\text { reporting } \\
\text { - Product rating } \\
\text { - Customer-to- } \\
\text { customer support }\end{array}$ & $\begin{array}{l}\text { Theoretical; } \\
\text { deductive } \\
\text { One-dimensional } \\
\text { table }\end{array}$ \\
\hline $\begin{array}{l}\text { Provide a useful } \\
\text { lens for viewing } \\
\text { crowdsourcing } \\
\text { (Brabham, 2012a, } \\
\text { 2013) }\end{array}$ & $\begin{array}{l}\text { Applications } \\
\text { (areas) }\end{array}$ & - Function & \begin{tabular}{|l|} 
- Knowledge \\
discovery and \\
management \\
- Broadcast search \\
- Peer-vetted creative \\
production \\
- Distributed human \\
intelligence
\end{tabular} & $\begin{array}{l}\text { Theoretical; } \\
\text { deductive } \\
\text { One-dimensional } \\
\text { table }\end{array}$ \\
\hline $\begin{array}{l}\text { Describe the } \\
\text { categories and the } \\
\text { characteristics of } \\
\text { crowdsourcing } \\
\text { applications } \\
\text { (Man-Ching et al., } \\
2011 \text { ) }\end{array}$ & Applications & - Function & $\begin{array}{l}\text { - Application (voting } \\
\text { system; } \\
\text { information sharing } \\
\text { system; game; } \\
\text { creative system) } \\
\text { - Algorithm } \\
\text { - Performance } \\
\text { dataset }\end{array}$ & $\begin{array}{l}\text { Theoretical; } \\
\text { deductive } \\
\text { Hierarchical } \\
\text { diagram }\end{array}$ \\
\hline $\begin{array}{l}\text { Understand } \\
\text { crowdsourcing } \\
\text { concept } \\
\text { (Zhao \& Zhu, } \\
\text { 2014) }\end{array}$ & Applications & $\begin{array}{l}\text { - Function } \\
\text { (Design \& } \\
\text { development; } \\
\text { Idea \& } \\
\text { consultant; Test } \\
\text { \& evaluation; } \\
\text { Other } \\
\text { - Context (Profit; } \\
\text { Non-profit) }\end{array}$ & $\begin{array}{l}\text { Typology defined by } \\
\text { the combination of } \\
\text { the variables }\end{array}$ & $\begin{array}{l}\text { Theoretical; } \\
\text { Two-dimensional } \\
\text { table }\end{array}$ \\
\hline \multicolumn{5}{|l|}{ Tasks } \\
\hline $\begin{array}{l}\text { Explore the } \\
\text { management } \\
\text { implications and } \\
\text { conceptualise the } \\
\text { phenomenon } \\
\text { (Rouse, } 2010)\end{array}$ & \begin{tabular}{|l|} 
Crowdsourcing \\
concept/tasks
\end{tabular} & $\begin{array}{l}\text { - Crowdsourcing } \\
\text { tasks } \\
\text { - Distribution of } \\
\text { benefits } \\
\text { - Forms of } \\
\text { motivation }\end{array}$ & $\begin{array}{l}\text { Taxonomy defined } \\
\text { by the combination } \\
\text { of the variables }\end{array}$ & $\begin{array}{l}\text { Theoretical; } \\
\text { deductive } \\
\text { Hierarchical } \\
\text { diagram }\end{array}$ \\
\hline
\end{tabular}




\begin{tabular}{|c|c|c|c|c|}
\hline $\begin{array}{c}\text { Classification } \\
\text { purpose }\end{array}$ & $\begin{array}{c}\text { Classification } \\
\text { objects }\end{array}$ & $\begin{array}{c}\text { Classification } \\
\text { variables }\end{array}$ & Categories/ Types & $\begin{array}{c}\text { Method } \\
\text { Means of } \\
\text { representation }\end{array}$ \\
\hline $\begin{array}{l}\text { Identify the } \\
\text { building blocks of } \\
\text { crowdsourcing } \\
\text { collective } \\
\text { intelligence } \\
\text { (Malone et al., } \\
2010)\end{array}$ & $\begin{array}{l}\text { Crowdsourcing } \\
\text { concept/tasks }\end{array}$ & $\begin{array}{l}\text { - What is being } \\
\text { crowdsourced } \\
\text { (Crowdsourcing } \\
\text { tasks) } \\
\text { - Who is } \\
\text { performing the } \\
\text { task } \\
\text { - Why people do } \\
\text { this } \\
\text { - How the task is } \\
\text { being done }\end{array}$ & $\begin{array}{l}\text { Framework defined } \\
\text { by the combination } \\
\text { of the variables }\end{array}$ & $\begin{array}{l}\text { Theoretical; } \\
\text { deductive } \\
\text { Two-dimensional } \\
\text { table }\end{array}$ \\
\hline $\begin{array}{l}\text { Create an } \\
\text { exhaustive } \\
\text { categorisation of } \\
\text { crowdsourcing } \\
\text { models } \\
\text { (Saxton et al., } \\
2013)\end{array}$ & $\begin{array}{l}\text { Crowdsourcing } \\
\text { models }\end{array}$ & \begin{tabular}{|l} 
- Outsourcing \\
task \\
(Crowdsourcing \\
tasks) \\
- Community user \\
role \\
- Level of \\
collaboration \\
- Managerial \\
control systems
\end{tabular} & $\begin{array}{l}\text { - Intermediary } \\
\text { - Citizen media } \\
\text { production } \\
\text { - Collaborative } \\
\text { software } \\
\text { development } \\
\text { - Digital goods sales } \\
\text { - Product design } \\
\text { - Peer-to-peer social } \\
\text { financing } \\
\text { - Consumer report } \\
\text { - Knowledge base } \\
\text { building } \\
\text { - Collaborative } \\
\text { science project }\end{array}$ & $\begin{array}{l}\text { Empirical (103 } \\
\text { websites); } \\
\text { inductive } \\
\text { Two-dimensional } \\
\text { table }\end{array}$ \\
\hline \begin{tabular}{|l|} 
Distinguish broad \\
classes of \\
crowdsourcing \\
practices and \\
characterize the \\
types of tasks \\
(Schenk \& \\
Guittard, 2009, \\
2011)
\end{tabular} & Tasks & \begin{tabular}{|l|} 
- Task complexity \\
(simple, \\
complex, \\
creative) \\
- Integration vs. \\
Selection
\end{tabular} & $\begin{array}{l}\text { Categories defined } \\
\text { by the combination } \\
\text { of the variables }\end{array}$ & $\begin{array}{l}\text { Theoretical; } \\
\text { deductive } \\
\text { Text }\end{array}$ \\
\hline $\begin{array}{l}\text { Classify } \\
\text { crowdsourcing in } \\
\text { terms of the types } \\
\text { of tasks for which } \\
\text { they are best } \\
\text { suited } \\
\text { (Nakatsu et al., } \\
2014)\end{array}$ & Tasks & \begin{tabular}{|l|} 
- Task complexity \\
(well-structured; \\
unstructured) \\
- Independent \\
tasks vs. \\
Interdependent \\
tasks \\
- Task \\
commitment \\
(high; low)
\end{tabular} & $\begin{array}{l}\text { Seven categories of } \\
\text { crowdsourcing, } \\
\text { defined by the } \\
\text { combination of the } \\
\text { variables }\end{array}$ & $\begin{array}{l}\text { Theoretical; } \\
\text { Two-dimensional } \\
\text { table with an } \\
\text { additional } \\
\text { dimension added } \\
\text { within each cell }\end{array}$ \\
\hline
\end{tabular}




\begin{tabular}{|c|c|c|c|c|}
\hline $\begin{array}{c}\text { Classification } \\
\text { purpose } \\
\text { Reference }\end{array}$ & $\begin{array}{c}\text { Classification } \\
\text { objects }\end{array}$ & $\begin{array}{c}\text { Classification } \\
\text { variables }\end{array}$ & Categories/ Types & $\begin{array}{c}\text { Method } \\
\text { Means of } \\
\text { representation } \\
\end{array}$ \\
\hline $\begin{array}{l}\text { Define the } \\
\text { concept of } \\
\text { crowdsourcing } \\
\text { inventive } \\
\text { activities } \\
\text { (Burger- } \\
\text { Helmchen \& } \\
\text { Pénin, 2010) } \\
\end{array}$ & $\begin{array}{l}\text { Crowdsourcing } \\
\text { task }\end{array}$ & $\begin{array}{l}\text { Level of } \\
\text { complexity }\end{array}$ & $\begin{array}{l}\text { - Complex } \\
\text { (crowdsourcing of } \\
\text { inventive activities) } \\
\text { - Routine } \\
\text { - Crowdsourcing of } \\
\text { content }\end{array}$ & $\begin{array}{l}\text { Theoretical; } \\
\text { deductive } \\
\text { Text }\end{array}$ \\
\hline $\begin{array}{l}\text { Understand the } \\
\text { dynamics of the } \\
\text { tasks } \\
\text { (Gadiraju, } \\
\text { Kawase, \& } \\
\text { Dietze, 2014) }\end{array}$ & Micro tasks & $\begin{array}{l}\text { - The goal of a } \\
\text { task }\end{array}$ & $\begin{array}{l}\text { - Information finding } \\
\text { - Verification \& } \\
\text { validation } \\
\text { - Interpretation \& } \\
\text { analysis } \\
\text { - Content creation } \\
\text { - Surveys } \\
\text { - Content access }\end{array}$ & $\begin{array}{l}\text { Empirical } \\
\text { (surveyed data) } \\
\text { One dimensional } \\
\text { table }\end{array}$ \\
\hline \begin{tabular}{|l} 
Propose an \\
integrated \\
typology \\
(Estellés-Arolas, \\
Navarro-Giner, \& \\
González-Ladrón- \\
de-Guevara, \\
$2015)$
\end{tabular} & $\begin{array}{l}\text { Crowdsourcing } \\
\text { type }\end{array}$ & - Task type & $\begin{array}{l}\text { - Crowd casting } \\
\text { - Crowd } \\
\text { collaboration } \\
\text { - Crowd content } \\
\text { - Crowdfunding } \\
\text { - Crowd opinion }\end{array}$ & $\begin{array}{l}\text { Theoretical; } \\
\text { deductive } \\
\text { Text }\end{array}$ \\
\hline \multicolumn{5}{|c|}{ Members of the crowd } \\
\hline $\begin{array}{l}\text { Examine } \\
\text { crowdsourcing } \\
\text { participation } \\
\text { levels } \\
\text { (Stewart et al., } \\
2010)\end{array}$ & $\begin{array}{l}\text { Crowd } \\
\text { participation }\end{array}$ & $\begin{array}{l}\text { - Quantity of } \\
\text { contribution } \\
\text { - The nature of } \\
\text { participants' } \\
\text { motivation }\end{array}$ & $\begin{array}{l}\text { - Super contributors } \\
\text { - Contributors } \\
\text { - Outliers }\end{array}$ & $\begin{array}{l}\text { Empirical; } \\
\text { inductive } \\
\text { Text }\end{array}$ \\
\hline \begin{tabular}{|l|} 
Create a typology \\
of crowdsourcing \\
participation \\
(Martineau, 2012)
\end{tabular} & $\begin{array}{l}\text { Crowd } \\
\text { participation }\end{array}$ & $\begin{array}{l}\text { - Level of } \\
\text { engagement } \\
\text { - Actions posed }\end{array}$ & $\begin{array}{l}\text { Four categories } \\
\text { were identified: } \\
\text { Communal, } \\
\text { Aspirer, Utilizer, } \\
\text { Lurker }\end{array}$ & $\begin{array}{l}\text { Empirical } \\
\text { (interviews); } \\
\text { inductive } \\
\text { Text }\end{array}$ \\
\hline $\begin{array}{l}\text { Understand } \\
\text { workers' } \\
\text { characteristics in } \\
\text { relation to the } \\
\text { work quality } \\
\text { (Gabriella Kazai } \\
\text { et al., 2011) }\end{array}$ & $\begin{array}{l}\text { Worker types } \\
\text { Relevance } \\
\text { label context }\end{array}$ & $\begin{array}{l}\text { - Behavioural } \\
\text { observations }\end{array}$ & $\begin{array}{l}\text { - Spammer } \\
\text { - Sloppy } \\
\text { - Incompetent, } \\
\text { - Competent } \\
\text { - Diligent }\end{array}$ & $\begin{array}{l}\text { Empirical; } \\
\text { inductive } \\
\text { Text }\end{array}$ \\
\hline
\end{tabular}




\begin{tabular}{|c|c|c|c|c|}
\hline $\begin{array}{c}\text { Classification } \\
\text { purpose }\end{array}$ & $\begin{array}{c}\text { Classification } \\
\text { objects }\end{array}$ & $\begin{array}{c}\text { Classification } \\
\text { variables }\end{array}$ & Categories/ Types & $\begin{array}{c}\text { Method } \\
\text { Means of } \\
\text { representation } \\
\end{array}$ \\
\hline $\begin{array}{l}\text { Detect spammers } \\
\text { (Vuurens \& De } \\
\text { Vries, 2012) }\end{array}$ & $\begin{array}{l}\text { Worker types } \\
\text { Quality } \\
\text { Relevance } \\
\text { Judgments }\end{array}$ & $\begin{array}{r}\text { - Behavioural } \\
\text { observations }\end{array}$ & $\begin{array}{l}\text { - Diligent workers } \\
\text { - Sloppy workers } \\
\text { - Random spammers } \\
\text { - Uniform spammers }\end{array}$ & $\begin{array}{l}\text { Theoretical; } \\
\text { deductive } \\
\text { Text }\end{array}$ \\
\hline \multicolumn{5}{|l|}{ Platforms } \\
\hline \begin{tabular}{|l} 
Classify \\
crowdsourcing \\
platforms \\
(Vukovic, 2009)
\end{tabular} & Platforms & $\begin{array}{l}\text { - Function } \\
\text { (design and } \\
\text { innovation; } \\
\text { development } \\
\text { and testing; } \\
\text { marketing and } \\
\text { sales; support) } \\
\text { - Mode } \\
\text { (competition; } \\
\text { integration) }\end{array}$ & $\begin{array}{l}\text { Categories defined } \\
\text { by the combination } \\
\text { of the variables }\end{array}$ & $\begin{array}{l}\text { Theoretical; } \\
\text { deductive } \\
\text { Two-dimensional } \\
\text { table }\end{array}$ \\
\hline $\begin{array}{l}\text { Give an inside } \\
\text { view of a } \\
\text { crowdsourcing } \\
\text { platform } \\
\text { (Hirth et al., } \\
2011 \text { ) }\end{array}$ & Platforms & $\begin{array}{l}\text { - Level of } \\
\text { specialisation }\end{array}$ & $\begin{array}{l}\text { - Specialised } \\
\text { - Non-specialised }\end{array}$ & $\begin{array}{l}\text { Theoretical; } \\
\text { deductive } \\
\text { Text }\end{array}$ \\
\hline $\begin{array}{l}\text { Offers a clear } \\
\text { picture of its core } \\
\text { and optional } \\
\text { features and how } \\
\text { they relate to each } \\
\text { other } \\
\text { (Hosseini et al., } \\
\text { 2014; Hosseini et } \\
\text { al., 2015b) }\end{array}$ & $\begin{array}{l}\text { Crowdsourcing } \\
\text { concept }\end{array}$ & $\begin{array}{l}\text { - Crowd } \\
\text { - Crowdsourcer } \\
\text { - Crowdsourced } \\
\text { task } \\
\text { - Crowdsourcing } \\
\text { platform }\end{array}$ & $\begin{array}{l}\text { Taxonomy defined } \\
\text { by the combination } \\
\text { of the variables and } \\
\text { detailed features of } \\
\text { each variable }\end{array}$ & \begin{tabular}{|l|} 
Theoretical; \\
inductive \\
Four one- \\
dimensional tables
\end{tabular} \\
\hline $\begin{array}{l}\text { Provide a global } \\
\text { picture of } \\
\text { crowdsourcing } \\
\text { systems on the } \\
\text { Web. } \\
\text { (Doan et al., } \\
2011 \text { ) }\end{array}$ & $\begin{array}{l}\text { Crowdsourcing } \\
\text { systems }\end{array}$ & \begin{tabular}{|l|} 
- Nature of \\
collaboration \\
- Type of target \\
problem. \\
- How to recruit \\
and retain users \\
- What \\
contributions \\
users can make \\
- How to combine \\
user \\
contributions to
\end{tabular} & $\begin{array}{l}\text { Classifications } \\
\text { defined by the } \\
\text { combination of the } \\
\text { variables }\end{array}$ & $\begin{array}{l}\text { Theoretical; } \\
\text { deductive } \\
\text { Text }\end{array}$ \\
\hline
\end{tabular}




\begin{tabular}{|c|c|c|c|c|}
\hline $\begin{array}{c}\text { Classification } \\
\text { purpose } \\
\text { Reference }\end{array}$ & \begin{tabular}{|c|} 
Classification \\
objects
\end{tabular} & $\begin{array}{c}\text { Classification } \\
\text { variables }\end{array}$ & Categories/ Types & $\begin{array}{c}\text { Method } \\
\text { Means of } \\
\text { representation }\end{array}$ \\
\hline & & $\begin{array}{l}\text { solve the target } \\
\text { problem? } \\
\text { - How to evaluate } \\
\text { users and their } \\
\text { contributions? } \\
\text { - Degree of } \\
\text { manual effort } \\
\text { - Role of human } \\
\text { users } \\
\text { - Standalone } \\
\text { versus } \\
\text { piggyback } \\
\text { architectures }\end{array}$ & & \\
\hline $\begin{array}{l}\text { Classify human } \\
\text { computation } \\
\text { systems } \\
\text { (Quinn \& } \\
\text { Bederson, 2011) }\end{array}$ & $\begin{array}{l}\text { Human } \\
\text { computer } \\
\text { system }\end{array}$ & $\begin{array}{l}\text { - Motivation } \\
\text { - Quality control } \\
\text { - Aggregation } \\
\text { - Human skill } \\
\text { - Process order } \\
\text { - Task request } \\
\text { cardinality }\end{array}$ & $\begin{array}{l}\text { - Human computer } \\
\text { system } \\
\text { - Crowdsourcing } \\
\text { - Social computation } \\
\text { - Collective } \\
\text { intelligent } \\
\text { - Data mining }\end{array}$ & $\begin{array}{l}\text { Theoretical; } \\
\text { inductive } \\
\text { Diagram }\end{array}$ \\
\hline $\begin{array}{l}\text { Identify meta- } \\
\text { characteristic of } \\
\text { crowdsourcing } \\
\text { information } \\
\text { systems } \\
\text { (Geiger, } \\
\text { Rosemann, Fielt, } \\
\text { \& Schader, 2012) }\end{array}$ & $\begin{array}{l}\text { Crowdsourcing } \\
\text { information } \\
\text { systems }\end{array}$ & $\begin{array}{l}\text { - Differentiation } \\
\text { between } \\
\text { contributions } \\
\text { (homogeneous; } \\
\text { heterogeneous) } \\
\text { - Value derived } \\
\text { from } \\
\text { contributions } \\
\text { (non-emergent; } \\
\text { emergent) }\end{array}$ & $\begin{array}{l}\text { - Crowd processing } \\
\text { systems } \\
\text { - Crowd rating } \\
\text { systems } \\
\text { - Crowd solving } \\
\text { systems } \\
\text { - Crowd creation } \\
\text { systems }\end{array}$ & $\begin{array}{l}\text { Theoretical; } \\
\text { Two-dimensional } \\
\text { table }\end{array}$ \\
\hline $\begin{array}{l}\text { Show how the } \\
\text { four categories } \\
\text { help firms } \\
\text { develop 'crowd } \\
\text { capital,' } \\
\text { (Prpić, Shukla, } \\
\text { Kietzmann, \& } \\
\text { McCarthy, 2015) }\end{array}$ & $\begin{array}{l}\text { Crowdsourcing } \\
\text { approaches }\end{array}$ & $\begin{array}{l}\text { - What type of } \\
\text { contributions } \\
\text { are required } \\
\text { from members } \\
\text { of the crowd } \\
\text { and } \\
\text { - How these } \\
\text { contributions } \\
\text { will collectively } \\
\text { help find a } \\
\text { solution to their } \\
\text { business } \\
\text { problem. }\end{array}$ & $\begin{array}{l}\text { - Crowd voting } \\
\text { - Idea crowdsourcing } \\
\text { - Micro-task } \\
\text { - Solution } \\
\text { crowdsourcing }\end{array}$ & $\begin{array}{l}\text { Theoretical; } \\
\text { deductive } \\
\text { Two-dimensional } \\
\text { diagram }\end{array}$ \\
\hline
\end{tabular}




\section{Appendix B. Ethics Approvals}

This research involves human participants in its research activities: case studies, experiments and focus groups. For conducting each of them, a separate human ethics application was approved by the Human Ethics Committee of the School of Information Management at Victoria University of Wellington, New Zealand. The three approvals of the Human Ethics applications are presented below.

\section{Approval of the Human Ethics Application: Interviews of the Case Study}

Reference:

0000022424

Principal Investigator:

Nguyen Hoang Thuan

Approval letter (Email):

Dear Thuan Nguyen,

Thank you for your application for ethical approval (To Establish Crowdsourcing as an Organizational Business Process: Interview, reference 0000021668), which has now been considered by the Information Management Sub-Committee of the Human Ethics Committee.

Your application is approved as of today.

You may wish to check whether there are any new comments on your application. To do this, click on the Comments button (it looks like a speech bubble). If any particular page has comments, they will be marked with a flag on the left hand side of the screen. To access them, navigate to the desired page, and then click on the Page Comments button (it looks like a speech bubble with a page behind it).

Best wishes with the research.

Information Management Human Ethics Sub-Committee

Note: Information sheet and consent form are presented below. 


\section{INFORMATION SHEET \\ (Interviews of the Case Study)}

I am Nguyen Hoang Thuan, a PhD student in the School of Information Management, Victoria University of Wellington, New Zealand. As part of this degree requirement, I am working on a thesis entitled "To Establish Crowdsourcing as an Organisational Business Process: An Exploratory Study". The aim of this research is to understand the establishment of business processes in organisational context. More precisely, this research focuses on exploring the main components (building blocks) and activities of this establishment, which is called business process crowdsourcing (BPC). This includes the following two steps. In the first step, a conceptual model was developed based on systematically reviewing the crowdsourcing literature. The second step further validates the model through case study, and thus your participation will provide the main source of data for the validation.

I am inviting coordinators and managers of crowdsourcing projects to participate in this study. More precisely, you will be interviewed and the interview session may take about 90 minutes. In this interview, I would like to know about your experience on crowdsourcing project(s), and your thinking on a proposed model that aims to support the establishment of BPC. I will take written notes and an audio record of the interview with your permission. Your participation is completely voluntary. Although it would be greatly appreciated if you would answer all questions as frankly as possible, you should not feel obliged to answer any questions that you find objectionable or that make you feel uncomfortable. Before we end the interview, you can withdraw any or all information that you have provided. You may also withdraw any or all information within one month after the interview.

Responses collected will form the basis of my research project and will be put into a written report on an anonymous basis. It will not be possible to be identified personally. Business names will also be masked. All material collected will be kept confidential. In case the interview is conducted in Vietnamese, a translator, who needs to sign a confidentiality agreement, may translate the collected data into English. No one other than myself, my supervisors, and the translator will have access to the collected materials. Ethics approval for the current research has been granted by the School of Information Management Human Ethics Committee.

As a result of the study, the thesis will be submitted for marking to the School of Information Management and deposited in the University Library. It is intended that one or more articles will be submitted for publication in scholarly journals. Audio recordings and interview transcripts will be destroyed five years after the end of the project.

If you have any questions or would like to receive further information about the project, please contact me at Thuan.Nguyen@ vuw.ac.nz (Phone: 0064 44637469) or my supervisors Associate Professor Pedro Antunes and Dr David Johnstone at the School of Information Management, Victoria University of Wellington, New Zealand.

Thank you.

Yours sincerely,

Nguyen Hoang Thuan 


\section{CONSENT FORM}

\section{(Interviews of the Case Study)}

I have been given and understood an explanation of this research project. I have had an opportunity to ask questions and have them answered to my satisfaction. I understand that I may withdraw myself (or any information I have provided) from this project (within one month after the interview) without having to give reasons or without penalty of any sort. I understand that any information I provide will be kept confidential to the researcher, the supervisors and the person who translates the audio recordings of our interview, the published results will not use my name, and that no opinions will be attributed to me in any way that will identify me. I understand that the business name may be disclosed only to the researcher, the supervisors and the translator during the data collection phase. In the thesis and all related conference and journal publications, the business name will be masked.

I understand that, where a translator has been used, the translator has signed a confidentiality agreement, agreeing not to disclose any details relating to identity or other information, to store material securely, and to destroy it after completing the translations.

I understand that the data I provide will not be used for any other purpose or released to others.

I understand that the audio recording of interviews will be destroyed five years after the end of the project.

Please indicate (by ticking the boxes below) which of the following apply:

$\square$ I agree to this interview being audio recorded.

I would like to receive a summary of the results when this research is completed.

(Please provide your email address:

Signed:

Name of participant:

Date: 


\section{Approval of the Human Ethics Application: Experiments}

Reference:

0000021948

Principal Investigator:

Nguyen Hoang Thuan

Approval letter (Email):

Dear Thuan Nguyen,

Thank you for your application for ethical approval (To Establish Crowdsourcing as an Organisational Business Process An Exploratory Study: A Decision Tool, reference 0000021948), which has now been considered by the Information Management Sub-Committee of the Human Ethics Committee.

Your application is approved as of today.

You may wish to check whether there are any new comments on your application. To do this, click on the Comments button (it looks like a speech bubble). If any particular page has comments, they will be marked with a flag on the left hand side of the screen. To access them, navigate to the desired page, and then click on the Page Comments button (it looks like a speech bubble with a page behind it).

Best wishes with the research.

Information Management Human Ethics Sub-Committee

Note: Information sheet and consent form are presented below. 


\section{INFORMATION SHEET}

\section{(Experiments)}

I am Nguyen Hoang Thuan, a PhD student in the School of Information Management, Victoria University of Wellington, New Zealand. As part of this degree requirement, I am working on a thesis entitled "To Establish Crowdsourcing as an Organisational Business Process: An Exploratory Study". The aim of this research is to understand the establishment of crowdsourcing as a business process in an organizational context, which is called business process crowdsourcing (BPC). More precisely, this research focuses on exploring the main activities of BPC and developing a decision tool supporting these activities. This includes the development of a conceptual model and an ontology of BPC. Based on the ontology, I have developed a tool supporting decision makers in their BPC process. This tool needs to be evaluated by experiments to verify its effectiveness. Thus, your participation will help in the evaluation of the tool.

In the experiments, you will act as a decision maker in a BPC process. Training will be given at the beginning of the experiments to help you become familiar with the concept of BPC. You will be asked to do two exercises. In one of them, the researcher will ask you to use a web-based tool to support your decision making on BPC process. You will use the provided tool for answer the questions in this and only this exercise. You will be asked to do the other exercise without the tool. Your answers in both exercises should be written out and submitted to the researcher(s). By the end of the exercises, you will be asked to evaluate the tool and its ability to support the decision making process. During the experiments, the researcher(s) will be happy to answer any questions that you might have about the BPC process. However, they will not answer any question related to the exercises.

Your participation in this project is completely voluntary, and you will neither be assessed nor judged according to the input you provide. You may choose to withdraw from your participation at any point during the experiment or within 1 month after the experiment without having to give reasons. In case you area student, your decision to participate or refusal to participate in the experiment or to withdraw during the experiment will not, in any way, affect your course results.

Responses collected will form the basis of my research project and will be put into a written report on an anonymous and aggregated basis. It will not be possible to be identified personally. All material collected will be kept confidential. Only my supervisors, Associate Professor Pedro Antunes and Dr David Johnstone and I will have access to the materials collected. In case where the collected data are in Vietnamese, a translator, who needs to sign a confidentiality agreement, may translate the collected data into English. Besides my supervisors, the translator, and me no other person will have 
access to the collected materials. Ethics approval for the current research has been granted by the School of Information Management Human Ethics Committee.

As a result of the study, the thesis will be submitted for marking to the School of Information Management and deposited in the University Library. It is intended that one or more articles will be submitted for publication in scholarly journals. Collected data will be destroyed five years after the end of the project.

If you have any questions or would like to receive further information about the project, please contact me at Thuan.Nguyen@vuw.ac.nz (Phone: 0064 44637469) or my supervisors Associate Professor Pedro Antunes and Dr David Johnstone at the School of Information Management, Victoria University of Wellington, New Zealand.

Thanks you.

Yours sincerely

Nguyen Hoang Thuan 


\section{CONSENT FORM}

\section{(Experiments)}

I have been given an Information Sheet about this research and understood the explanations given. I have had the opportunity to ask questions and have them answered to my satisfaction.

I understand that my participation in this project is completely voluntary. I also understand that I may withdraw from my participation during the experiment without having to give reasons. In this case, I may choose that any information I have provided as part of my participation will be excluded from the data collection and analysis. I understand that I will not be assessed nor judged for my contribution to this research project. In particular, I understand that my decision to participate, or refusal to participate in the experiment or to withdraw during the experiment will not, in any way, affect my course results.

I understand that the data collected in this research may be used for research reports, including a PhD thesis, a conference paper, or a journal article. I understand that the raw data will be reported on an anonymous and aggregated basis. It will not be possible to be identified personally. All material collected will be kept confidential.

I understand that the data I provide will not be used for any other purpose or released to others.

I understand that the collected data will be destroyed five years after the end of the project.

Please indicate (by ticking the boxes below) which of the following apply:

$\square$ I would like to receive a summary of the results when this research is completed.

(Please provide your email address:

Signed:

Name of participant:

Date: 


\section{Approval of the Human Ethics Application: Focus Groups}

Reference:

0000022424

Principal Investigator:Nguyen Hoang Thuan

Approval letter (Email):

Dear Thuan Nguyen,

Thank you for your application for ethical approval (To Establish Crowdsourcing as an Organisational Business Process An Exploratory Study: A Decision

Tool, reference 0000022424 ), which has now been considered by the

Information Management Sub-Committee of the Human Ethics Committee.

Your application is approved as of today.

You may wish to check whether there are any new comments on your application. To do this, click on the Comments button (it looks like a speech bubble). If any particular page has comments, they will be marked with a flag on the left hand side of the screen. To access them, navigate to the desired page, and then click on the Page Comments button (it looks like a speech bubble with a page behind it).

Best wishes with the research.

Information Management Human Ethics Sub-Committee

Note: Information sheet and consent form are presented below. 


\section{INFORMATION SHEET}

(Focus Groups)

I am Nguyen Hoang Thuan, a PhD student in the School of Information Management, Victoria University of Wellington, New Zealand. As part of this degree requirement, I am working on a project entitled "To Establish Crowdsourcing as an Organisational Business Process: A Decision Tool". The aim of this research is to understand the establishment of an business process in an organizational context. More precisely, this research focuses on exploring the main components (building blocks) and activities of this establishment, which is called business process crowdsourcing (BPC). This includes the following two steps. In the first step, a decision tool was already developed based on systematically reviewing the crowdsourcing literature. The second step further validates the tool through focus groups, and thus your participation will provide the main source of data for the validation.

I am inviting crowdsourcing practitioners, researchers and experts to participate in this focus group. More precisely, you will participate in a group discussion, focusing on a tool supporting the crowdsourcing decisions. A fifteen-minute seminar will be given at the beginning of the focus group to help you become familiar with the tool. The focus group session may take about 60-90 minutes. In this focus group, I would like to know your thinking on a proposed decision tool, its utility, and possible improvement. I will take written notes and video record of the focus group with your permission. Your participation is completely voluntary. You have the right to withdraw at any point up to the start of the focus group session or during the focus group session, but any data collected up to that point of withdrawal cannot be removed.

Responses collected will form the basis of my research project and will be put into a written report on an anonymous basis. It will not be possible to be identified personally. Organisation names will also be masked. All material collected will be kept confidential. No one other than myself and my supervisors will have access to the collected materials. Ethics approval for the current research has been granted by the School of Information Management Human Ethics Committee.

As a result of the study, the thesis will be submitted for marking to the School of Information Management and deposited in the University Library. It is intended that one or more articles, conference presentations, and/or conference papers will be submitted for publication in scholarly journals and conferences. Video recording will be destroyed five years after the end of the project.

If you have any questions or would like to receive further information about the project, please contact me at Thuan.Nguyen@vuw.ac.nz (Phone: 0064 44635504) or my supervisors Associate Professor Pedro Antunes and Dr David Johnstone at the School of Information Management, Victoria University of Wellington, New Zealand.

Thank you

Yours sincerely,

Nguyen Hoang Thuan 


\section{CONSENT FORM}

\section{(Focus Groups)}

I have been given and understood an explanation of this research project. I have had an opportunity to ask questions and have them answered to my satisfaction. I understand that I have the right to withdraw at any point up to the start of the focus group session or during the focus group session, but any data collected up to that point of withdrawal cannot be removed.

I understand that any information I provide will be kept confidential to the researcher, and the supervisors. According to confidentiality ground rules, I will not release the discussion in the focus group to others. The published results will not use my name, and no opinions will be attributed to me in any way that will identify me. I understand that the organisation name may be disclosed only to the researcher and the supervisors during the data collection phase. In the thesis and in all related conference and journal publications, the organisation name will be masked.

I understand that the data I provide will not be used for any other purpose or released to others.

I understand that the video recording of the focus group will be destroyed five years after the end of the project.

Please indicate (by ticking the boxes below) which of the following apply:

$\square$ I understand that the focus group will be video recorded.

$\square$ I would like to receive a summary of the results when this research is completed.

(Please provide your email address:

Signed:

Name of participant:

Date: 


\section{Appendix C. Coding Form}

The following coding form was used to extract data in the scoping knowledge sources.

\section{General information}

\begin{tabular}{|l|c|}
\hline \multicolumn{1}{|c|}{ Data item } & \multicolumn{1}{c|}{ Value } \\
\hline Article & \\
\hline Year of publication & $\square$ Journal \\
\hline Publication type & $\square$ Conference \\
\hline Date of coding & \\
\hline Note & \\
\hline
\end{tabular}

\section{Conceptual stages, topics and findings}

\begin{tabular}{|c|c|c|c|c|}
\hline Data item & \multicolumn{4}{|c|}{ Value } \\
\hline Main stages & \multicolumn{4}{|c|}{$\begin{array}{l}\square \text { Decision to crowdsource } \\
\square \text { Design } \\
\square \text { Configuration }\end{array}$} \\
\hline \multirow{3}{*}{$\begin{array}{l}\text { Topics } \\
\text { (Iterative } \\
\text { update) }\end{array}$} & \multicolumn{3}{|c|}{$\square$ Workflow design } & $\square$ Task design \\
\hline & \multicolumn{3}{|c|}{$\square$ Quality control } & $\square$ Task design \\
\hline & \multicolumn{4}{|l|}{ Other topics: } \\
\hline \multirow{3}{*}{$\begin{array}{l}\text { Research } \\
\text { findings }\end{array}$} & Main findings & & & \\
\hline & Other findings & & & \\
\hline & $\begin{array}{l}\text { Knowledge } \\
\text { generated from } \\
\text { the findings }\end{array}$ & \multicolumn{3}{|c|}{$\begin{array}{l}\square \text { Generalizable to other situation } \\
\square \text { Generalizable only to a similar situation }\end{array}$} \\
\hline
\end{tabular}

\section{Practical outcomes}

\begin{tabular}{|c|c|c|c|c|c|}
\hline Data item & \multicolumn{5}{|c|}{ Value } \\
\hline \multirow{5}{*}{$\begin{array}{l}\text { Crowdsourcing } \\
\text { contexts }\end{array}$} & \multirow[b]{2}{*}{ Applications } & $\begin{array}{l}\square \text { Profit vs. } \\
\text { Non-profit }\end{array}$ & $\square$ Profit & \begin{tabular}{|l|} 
Non- \\
Profit
\end{tabular} & $\square$ N/A \\
\hline & & Function & \begin{tabular}{|l}
$\square$ Design \\
$\square$ Idea \& \\
$\square$ Test \& \\
$\square$ Other \\
\end{tabular} & $\begin{array}{l}\text { \& Developr } \\
\text { Consultant } \\
\text { Evaluation }\end{array}$ & ment \\
\hline & \multirow[t]{2}{*}{ Tasks } & $\begin{array}{l}\text { Participation } \\
\text { mode }\end{array}$ & \multicolumn{3}{|c|}{\begin{tabular}{|l}
$\square$ Individual (Integrative) \\
$\square$ Competitive (Selective) \\
$\square$ N/A
\end{tabular}} \\
\hline & & Complexity & $\square$ Simple & $\square$ Skilled & $\square$ N/A \\
\hline & Other contexts & & & & \\
\hline \multicolumn{2}{|c|}{ Practical recommendations } & & & & \\
\hline
\end{tabular}




\section{Appendix D. Interview Protocol}

Two case studies were conducted to evaluate the process model. The following protocol was used to interview participants in these case studies.

\section{Preparation}

Before the interview begins, I will introduce the research and terms used for the research to the participant. The introduction will include:

i. Research aims

ii. Terms used for the research

I will then interview the participant with the following questions:

\section{General information about their crowdsourcing projects and experience.}

1. Tell me about the crowdsourcing project(s) that you have already participated in

2. Can you describe your roles in the(se) project(s)?

a. For how long?

3. Apart from you, who else was involved in planning and conducting the project?

4. How did you prepare for the project?

5. Please describe the process of the crowdsourcing project

a. Do you use any models, frameworks, or guidance that help you design and manage your project(s)?

\section{Delivery of the conceptual model to the participant}

1. The following model will be presented to the participant.

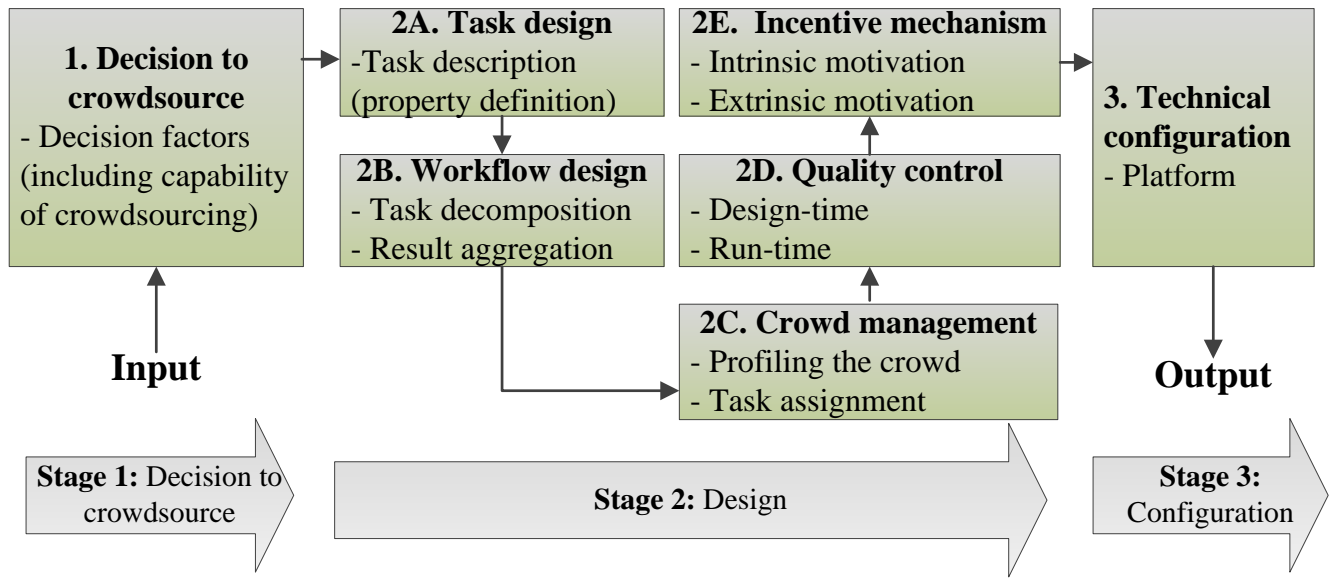

2. The investigator will shortly explain the components of the model. 


\section{Crowdsourcing project(s) in regard to the components of the model}

1. Considering the project you participated in, why do you choose crowdsourcing for your project?

2. What factors influence this decision?

3. How do you design your crowdsourcing task/contest/micro task?

4. What is the workflow of the crowdsourcing process? (Or how many phases were conducted in the project?)

5. Does the project have any methods to search and match tasks to the crowd?

6. How does the project manage and coordinate the crowd?

a. Is there any communication between the project/organisations and the crowd?

b. Does the project know the targeted crowd in advance?

7. What incentive mechanisms are adopted for the project?

a. Intrinsic motivation

b. Extrinsic motivation

c. What are the main reasons an individual will participate in the project?

8. Does the project have any methods to ensure quality of results?

a. Does the project use experts/voting to assess the submission quality?

b. Are there any cheating submissions, e.g. submission copied from the Internet? How do you identify them?

9. From a technical perspective, what platform (channel) was adopted for the project?

a. AMT/ Threadless?

10. Overall, do you think that your project is effective, in terms of cost and benefit?

a. What was especially surprising you?

b. What was unexpected during the project?

\section{Model evaluation}

1. What do you think about the components of the model?

a. Out of these components, what are the most important ones in your crowdsourcing project?

2. What do you think about the sequence of the model components?

3. How do you evaluate the given model for conceptualising crowdsourcing project(s), in terms of:

a. Conceptualisation?

b. Communication?

4. Did/does your project(s) have any additional activities that the model does not present?

a. Can you explain these activities? 
5. Did/does the model present any activities that were not performed in your projects?

a. Can you explain these activities?

6. Will you want to use this model for your future crowdsourcing project(s)? Why/why not? 


\section{Appendix E. Ontology Vocabulary}

An ontology dictionary summarises and defines the concepts related to BPC. The dictionary structures the BPC concepts as a set of parent and child concepts. The dictionary is available in a file named Ontology Dictionary of BPC.docx. This file can be viewed and downloaded from the following location: Dropbox 


\section{Appendix F. Experimental Sheet}

Demographic information. Please complete the following demographic information

Name:

\begin{tabular}{|c|c|c|c|}
\hline Gender: & $\square$ Male & & $\square$ Female \\
\hline Major: & $\square$ IT/IS & & $\square$ Industrial Manageme \\
\hline Year of study: & $\square$ First & $\square$ Second & $\square$ Third \\
\hline
\end{tabular}

Exercise 1: Please read the four scenarios and answer each of the following questions (30 minutes)

Reference number

Group: $\quad \square$ Using the tool $\quad \square$ Without the tool

Scenario 1: Company $X$ has a project to digitise clients' credit information (payment history, credit cards, mortgages, car finance, and hire purchases). In this project, the main tasks are to digitise 500 scanned reports, which requires general computer skills. The estimated time for each task is five minutes. These tasks are independent and can be performed individually.

- Should this task be crowdsourced?
$\square$ Yes
No
No Idea

Please provide from 1 to 3 reasons for your answer.

Reasons:

Scenario 2: To control traffic in Wellington city (or Ho Chi Minh city), organisation Y has a project with the main task being to collect traffic information. In this task, traffic data are collected through a smart phone application. The application enables the users to report information about the location, GPS point, pictures of the traffic flows in the location, and users' judgement on the traffic flows. The target of the project is to collect a data set of 10,000 records. The project has ten employees, and the budget allocated for it is 10,000 NZD.

- Should this task be crowdsourced?
Yes
$\square$ No
No Idea

Please provide from 1 to 3 reasons for your answer.

Reasons: 
Scenario 3: $\mathrm{Z}$ is an architecture firm with several experienced architects. The firm has a project to design bus stops, which will be installed in the whole Wellington city (or Ho Chi Minh city). The requirements of the design include usefulness, innovation, and friendliness. More importantly, the design needs to be accepted by the citizens. The design can be either a completed design in detail or an illustrated sketch. Budget allocated for the project is 10,000 NZD.

- Should this design task be crowdsourced?

$\square$ Yes $\quad \square$ No $\quad \square$ No Idea

Please provide from 1 to 3 reasons for your answer.

Reasons:.

Scenario 4: U Hospital is a national hospital dedicated to cancer treatment, which focuses on predicting cancer and tailoring treatments to each patient. Every week, the hospital receives 1,000 clinical records from the local hospitals with the requests for cancer prediction. The main tasks in cancer prediction include examining the classification of cell mass biopsy images, determining the malignancy of tissue samples, and identifying cancer cells. With many such tasks, the hospital is looking for additional workforce to handle the image examination.

- Should this mange examination task be crowdsourced?

$\square$ Yes $\quad \square$ No $\quad \square$ No Idea

Please provide from 1 to 3 reasons for your answer.

Reasons:

(Note: You may ask for more paper) 
Exercise 2: Please read the two scenarios and answer each of the following questions (30 minutes)

$$
\text { Reference number }
$$

Group:

Using the tool

Without the tool

Scenario 5: Organisation A has 10,000 pictures about wild animals that were captured by motiontriggered cameras in 40 wild locations in New Zealand. This organisation sets up a project aiming at identifying the animals and their names in the pictures (if there are any). As the number of tasks is very large, the organisation decides to crowdsource. The main job in the project is to view the picture, identify whether there are any animals in the picture, and input the animal name (if known). The budget for the project is 2,000 NZD.

- Should this project divide tasks into sub-tasks?

$$
\square \text { Yes } \square \text { No }
$$

No Idea

Please provide the reasons for your answer.

Reasons:

What is the workflow to establish the project (step $1,2, \ldots$ )?

Workflow:

Scenario 6: The Crowd Logo project aims at designing a logo for a technical university. This logo should represent the spirit of the university, which includes open mind, creativity, and leading technology. To collect expertise from outside, the project decides to crowdsource and invites the crowd to join a logo design competition. Everyone can submit their design, consisting of a logo, a slogan, and an explanation on the meaning of the logo. The budget for the project is 5,000 NZD.

- How to motivate the crowd to participate in the competition?

Way(s) to motivate:

- Normally, this kind of competition receives a large number of submissions. How can this project assess the quality of the submissions?

Quality assessment:

\footnotetext{
${ }^{8}$ The reference number is re-printed, and is the same for exercise 1 and exercise 2
} 
Evaluation: Based on your interaction with the tool, please circle the appropriate number after each statement that best reflects your opinion (10 minutes).

\section{1 =strongly disagree $\quad 2$ =disagree 3 =neither agree nor disagree 4 =agree 5 =strongly agree}

- Using the tool allows me to better answer the questions in the exercises $\quad \begin{array}{lllll}1 & 2 & 3 & 4 & 5\end{array}$

- Using the tool allows me to faster answer the questions in the exercises $\quad \begin{array}{lllll}1 & 2 & 3 & 4 & 5\end{array}$

- Using the tool allows me to better understand the questions in the exercises $\begin{array}{llllll}1 & 2 & 3 & 4 & 5\end{array}$

- I find using the tool useful $\quad \begin{array}{lllll}1 & 2 & 3 & 4 & 5\end{array}$

- Other comments:

(Note: You may ask for more paper) 


\section{Appendix G. Partial Focus Group Script}

\section{Tasks before the focus group}

Before the focus group:

- Arrange furniture, set up video recorder, set up computers to introduce the tool

- Great and chat with people as they come in.

- Encourage them to try the tool and fill consent forms while they wait.

\section{Start the focus group}

I will then start the focus group with the following activities:

- Introduce the research and terms used in the research to the participants.

- Introduce the crowdsourcing tool with two main functions

- Participant introduction (Let's begin with introductions. Please tell us your name and a brief description of your crowdsourcing projects. I'll start; then we can go around the room)

\section{Discussion questions (will be adapted during the discussion)}

- The participants are shown a crowdsourcing scenario about the crowdsourcing contest for logo design.

- They are instructed to perform the decision tasks without and then with the tool Note: allow conversation to flow - the goal of focus groups is to stimulate conversation from the comments of other participants. Each question should have about 5-10 minutes to discuss

Questioning route:

1. In the first scenario, how many decision factors that can be identified?

2. What do you think about the functions of the tool?

3. Do you find this tool useful? In what way?

a. Did the tool change your answers?

4. In the second scenario:

a. What does your described workflow look like?

b. How do you set up quality control for the crowdsourcing project?

5. What do you think about the information provided by the tool?

a. Would it be beneficial to you?

b. Would it support your decision? 
6. Overall, how do you rate your confidence levels in addressing the scenarios with/without the tool?

7. Is the tool easy or complicated to use?

8. What are your suggestions to improve the functions of the tool?

9. Are there any functions or decisions that we should add into the tool?

10. Any final thoughts or comments?

Thank everybody for participating —invite everyone to join for teatime. 